\title{
FLOW-INDUCED MICRO- AND NANO-FIBER SUSPENSIONS IN SHORT-FIBER REINFORCED COMPOSITE MATERIALS PROCESSING
}

A Dissertation presented to

the Faculty of the Graduate School

at the University of Missouri

In Partial Fulfillment

of the Requirements for the Degree

Doctor of Philosophy

\author{
by \\ DONGDONG ZHANG
}

Dr. Douglas E. Smith, Dissertation Supervisor

May 2013 
The undersigned, appointed by the Dean of the Graduate School, have examined the dissertation entitled:

\section{FLOW-INDUCED MICRO- AND NANO-FIBER SUSPENSIONS IN SHORT-FIBER REINFORCED COMPOSITE MATERIALS PROCESSING}

presented by Dongdong Zhang, a candidate for the degree of Doctor of Philosophy and hereby certify that, in their opinion, it is worthy of acceptance.

Dr. Douglas E. Smith

Dr. Frank Pai

Dr. Yuwen Zhang

Dr. Stephen Montgomery-Smith

Dr. David A. Jack 


\section{ACKNOWLEDGMENTS}

The Ph.D. study has been a challenging, unforgettable and enjoyable experience for me and it would not have been possible to finish without the support and help that I received from many people.

First and foremost, it is with immense gratitude that I acknowledge the guidance and help of my advisor Dr. Douglas Smith. I have been working with Dr. Smith for about four and half years. He continually and convincingly conveyed a spirit of adventure in regard to research and future career, and an excitement of teaching. Dr. Smith gave me all opportunities to train and develop myself in research abilities and teaching skills. Without the constant encouragement, guidance and help from Dr. Smith, this dissertation would not have been possible.

Special thanks are given to my committee members, Dr. Frank Pai, Dr. Yuwen Zhang, Dr. Stephen Montgomery-Smith and Dr. David Jack, for the help and precious discussions. I would also like to thank Dr. Frank Feng for the long-term help since I joined the Department of Mechanical and Aerospace Engineering.

I am also indebted to my colleagues who supported me. I want to thank Jamie, Roxana, Tyler, Nikhil, Brad, Shuyi, Yunpeng, Sunxin, Weijun and many others who have given me lots of help.

The financial supports from the National Science Foundation through CMMIMPM (Grant No.0727399) and the University of Missouri Research Council (Grant No.URC-11-076) are gratefully acknowledged.

Last but not least, I owe my deepest gratitude to my parents, my parents-in-law, my wife, Na and my one-year-old son, Jayden. Without their love, support, care and understanding, I cannot finish my research work and complete this dissertation. Thank them so much from the bottom of my heart. 


\section{TABLE OF CONTENTS}

ACKNOWLEDGMENTS ................ ii

LIST OF TABLES $\ldots \ldots \ldots \ldots \ldots \ldots$ vii

LIST OF FIGURES $\ldots \ldots \ldots \ldots \ldots \ldots \ldots \ldots$ ix

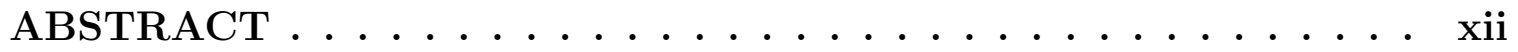

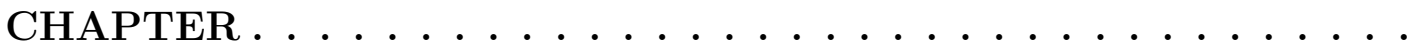

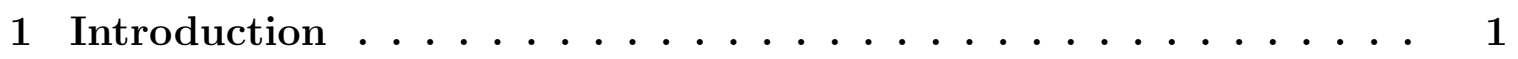

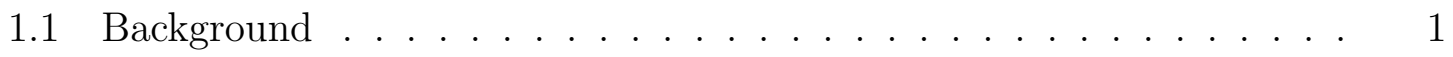

1.2 Research motivation . . . . . . . . . . . . . . . . . 9

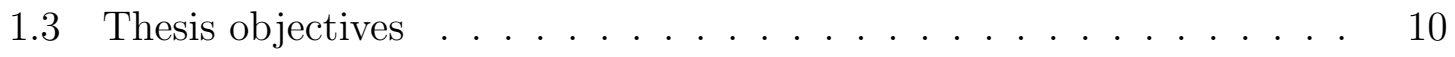

1.4 Organization of the thesis $\ldots \ldots \ldots \ldots \ldots$

2 Numerical Evaluation of the Motion of Fibers with Various Shapes Using Commercial FEM Solver . . . . . . . . . . . . . . 14

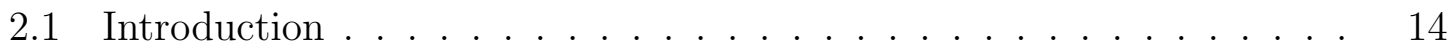

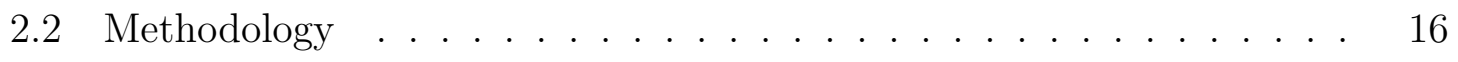

2.2 .1 Fluid dynamics simulation . . . . . . . . . . . . 16

2.2 .2 Computing fiber velocities $\ldots \ldots \ldots \ldots \ldots$

2.2 .3 Evolution of fiber motion . . . . . . . . . . . . . . 19

2.2.4 Summary of numerical approach . . . . . . . . . . . 20

2.3 Role of fiber shape $\ldots \ldots \ldots \ldots$

2.4 Implementation and examples . . . . . . . . . . . . . . 22

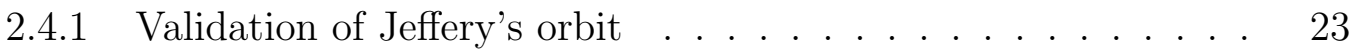

2.4.2 Equivalent aspect ratio - general axisymmetric fiber . . . . . 27 
2.4.3 Validation of equivalent aspect ratio . . . . . . . . . . . 32

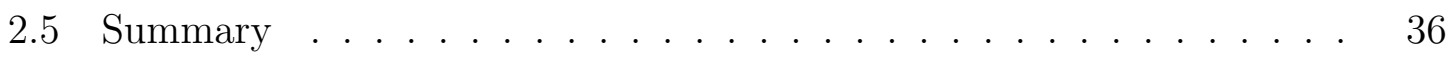

3 Stand-Alone FEM Package Using Analytical Jacobian and Transformed Essential Boundary ............... 38

3.1 Introduction . . . . . . . . . . . . . . . . . . . . . . . 38

3.2 Chwang's model for Poiseuille flows . . . . . . . . . . . . . . . . . . 41

3.3 FEM-based discrete fiber simulation . . . . . . . . . . . . . . . 44

3.3.1 Definition of fiber orientation/position . . . . . . . . . . 44

3.3.2 Governing equations ................ 45

3.3.3 Finite element analysis . . . . . . . . . . . . . . . . . . . 46

3.3.4 Searching fiber velocities . . . . . . . . . . . . . . . 48

3.3.5 Tracking fiber motion with transformed boundary . . . . . . 50

3.3.6 Summary of the proposed methodology . . . . . . . . . . 52

3.4 Implementation and examples . . . . . . . . . . . . . . . . 52

3.4.1 Validation of the proposed methodology . . . . . . . . . 53

3.4.2 Fiber motion in various homogeneous flows . . . . . . . . . 55

3.4.3 Fiber motion in Poiseuille flows . . . . . . . . . . . . . 58

3.5 Summary . . . . . . . . . . . . . . . . 70

4 Brownian Dynamics Simulation of Nano-Fiber Suspensions for Nano-Fiber Composites Processing . . . . . . . . . 72

4.1 Introduction . . . . . . . . . . . . . . . . . . 72

4.2 FEM-based Brownian dynamics simulation . . . . . . . . . . 73

4.3 Implementation and examples . . . . . . . . . . . . . . 78

4.3.1 Comparison of two- and three-dimensional fiber motions . . . 78

4.3.2 Brownian motions of a sphere with $r_{e}=1 \ldots \ldots$. . . . 80 
4.3.3 Brownian motion of an ellipse with $r_{e}=3 \ldots . . . . . .884$

4.3.4 Brownian motion of an ellipse with $r_{e}=10 \ldots . . . . \quad 87$

4.4 Summary . . . . . . . . . . . . . . . . . . . 87

5 Physical Understanding of Fiber-Fiber Interactions and Bounded Fluid Domain on Fiber Motion . . . . . . . . . . . . 89

5.1 Introduction . . . . . . . . . . . . . . . . . . . . . 89

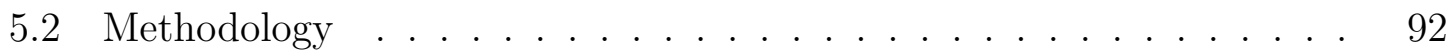

5.2.1 Finite element model . . . . . . . . . . . . . . 93

5.2.2 Hydrodynamic forces and torques . . . . . . . . . . . . . . 94

5.2.3 Updating fiber orientations and positions . . . . . . . . . 96

5.3 Effect of the bounded fluid domain . . . . . . . . . . . . . 96

5.4 Implementation and examples . . . . . . . . . . . . . . . . . . 99

5.4.1 Fiber-fiber interaction between two fibers . . . . . . . . . . 99

5.4.2 Effect of multiple fiber suspensions . . . . . . . . . . . . . . . 110

5.4.3 Fiber motion near a stationary wall . . . . . . . . . . 115

5.5 Summary . . . . . . . . . . . . . . . . . 116

6 Summary and Concluding Remarks .............. 119

6.1 Summary of the dissertation . . . . . . . . . . . . . . . . 119

6.2 Thesis contributions . . . . . . . . . . . . . . . 121

6.3 Future work . . . . . . . . . . . . . . . . . 123

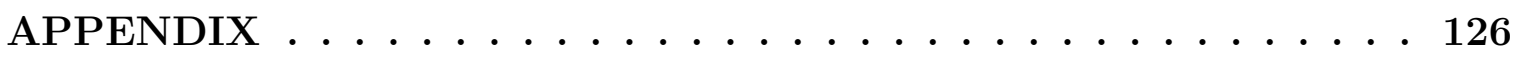

A Three-dimensional Finite Element Method in Solving Fiber Motion within a Viscous Flow .............. 126

A.1 Basis of fluid mechanics _. . . . . . . . . . . . . . . 126

A.1.1 Governing equations ............... 126 
A.1.2 Boundary conditions . . . . . . . . . . . . . . . . . 127

A.2 Finite Element Method for Newtonian fluids . . . . . . . . . . . . . . 127

A.2.1 Weak form of governing equations . . . . . . . . . . . 128

A.2.2 Discretization . . . . . . . . . . . . . . . 129

A.2.3 System of equations . . . . . . . . . . . . . . . . . . . . . 131

A.2.4 Gauss quadrature for tetrahedral element . . . . . . . . . . . 135

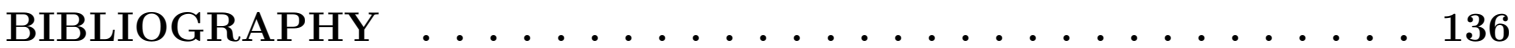

VITA ......................... 148 


\section{LIST OF TABLES}

Table $\quad$ Page

2.1 Parameters to simulate an ellipsoidal fiber motion . . . . . . . . . 23

2.2 Comparison of the results between Jeffery's theory and our approach 26

2.3 Equivalent aspect ratios of ellipsoidal fibers . . . . . . . . . . . . . . 27

2.4 Comparison of two bead-chain models . . . . . . . . . . . . . . . 31

2.5 Comparison of equivalent aspect ratios of fibers with various shapes . 32

3.1 Dimensionless parameters to simulate an ellipsoidal fiber motion . . . 53

3.2 Comparison of Jeffery's data and the numerical data . . . . . . . 53

3.3 Efficiency of the calculation of the proposed FEM package . . . . . 55

4.1 Parameters to simulate the motions of micro- and nano-fibers . . . . 81

4.2 Fiber orientations with different Péclet numbers $\left(r_{e}=1\right) \ldots 2$

4.3 Fiber positions with different Péclet numbers $\left(r_{e}=1\right) \ldots . . . . \quad 84$

4.4 Fiber sizes and Péclet numbers used in the simulation . . . . . . . . . 84

4.5 Fiber orientations with different Péclet numbers $\left(r_{e}=3\right) \ldots . . \quad 85$

4.6 Fiber positions with different Péclet numbers $\left(r_{e}=3\right) \ldots . . . \quad 85$

4.7 Fiber motions with different Péclet numbers $\left(r_{e}=10\right) \ldots . . . \quad 88$

5.1 Parameters to simulate the motions of two ellipsoidal fibers . . . . . . 101

5.2 Comparison of time periods of fiber rotations. . . . . . . . . . . . . 102

5.3 Parameters to simulate fiber motion in a bounded fluid domain . . . 110 
5.4 Equivalent aspect ratios of fibers in a bounded fluid domain with various sizes . . . . . . . . . . . . . . . . . . . . . 112 


\section{LIST OF FIGURES}

Figure

Page

1.1 Injection molding process . . . . . . . . . . . . . . 2

1.2 Fiber orientation of an ellipsoidal fiber defined in Jeffery's theory . . 3

1.3 Jeffery's orbit with different orbit constants $\ldots \ldots \ldots \ldots$

1.4 Deviation of orientation tensor in experimental data from theory . . 10

2.1 Finite element model in COMSOL . . . . . . . . . . . . . . 16

2.2 Torques on an ellipsoidal fiber fixed in two directions . . . . . . . . 21

2.3 Finite element model of a single ellipsoidal fiber in a simple shear flow 23

2.4 Three-dimensional single fiber orientation in half period . . . . . . . 24

2.5 Three-dimensional single fiber translation in half period . . . . . . 25

2.6 Torques on a cylindrical fiber fixed in two directions . . . . . . . 28

2.7 Equivalent aspect ratios of cylinders . . . . . . . . . . . . . . . . 29

2.8 Torques on a bead-chain fiber fixed in two directions . . . . . . . 30

2.9 Equivalent aspect ratios of bead-chain fibers . . . . . . . . . 30

2.10 Evolution of a cylindrical fiber with $r_{e}=6$ and an ellipsoidal fiber with $r_{e}=4.8 \ldots \ldots \ldots \ldots \ldots \ldots \ldots \ldots \ldots \ldots \ldots \ldots \ldots \ldots \ldots$

2.11 Evolution of a bead-chain fiber with $r_{e}=6$ and an ellipsoidal fiber with $r_{e}=5.4 \ldots \ldots \ldots \ldots \ldots \ldots \ldots \ldots \ldots \ldots \ldots \ldots$

2.12 Comparison of the motions of ellipsoidal, cylindrical and bead-chain fibers with the same geometric aspect ratio . . . . . . . . . 
3.1 Two types of Poiseuille flows . . . . . . . . . . . . . . . . . 41

3.2 The decomposition of a simple shear flow into the coordinate system with fiber centroid at the origin . . . . . . . . . . . . 43

3.3 Definition of fiber orientation and position in the proposed approach . 44

3.4 Finite element model of a single fiber with boundary conditions . . . 46

3.5 Numerical results of three-dimensional ellipsoidal fiber motion . . . . 54

3.6 Fiber orientation in a rotational flow $\ldots \ldots \ldots \ldots . \ldots 57$

3.7 Fiber orientation in a stretching flow $\ldots \ldots \ldots \ldots$

3.8 Fiber motion in the quadratic flow component with different initial

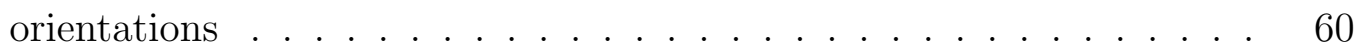

3.9 Fiber's linear motion in a quadratic flow component . . . . . . . . . 62

3.10 Relationship between the pressure gradient and fiber's linear velocity 63

3.11 Fiber rotation in a general plane Poiseuille flow . . . . . . . . . 64

3.12 Fiber translation in a general plane Poiseuille flow . . . . . . . . 65

3.13 Change of fiber's linear velocity in a circular Poiseuille flow . . . . . 67

3.14 Fiber orientation in a circular Poiseuille flow . . . . . . . 68

3.15 Fiber translation in a circular Poiseuille flow . . . . . . . . . . 69

4.1 Comparison of three-dimensional FEM and two-dimensional FEM . . 79

4.2 Disturbance of fiber motion on fluid velocity, pressure and stress around fiber surface without Brownian motion $\ldots \ldots \ldots$. . . . 81

4.3 Distribution of fiber orientation for small and large Péclet numbers . 83

4.4 Disturbance of fiber motion on fluid velocity, pressure and normal stress difference around fiber surface with Brownian motion . . . . . . . 86

5.1 Finite element model of multiple fibers with boundary conditions . . 93

5.2 The effect of multiple fiber interactions $\ldots \ldots \ldots \ldots \ldots$

5.3 Torques on a fiber fixed in a bounded fluid domain $\ldots \ldots$. . . 97 
5.4 Uniform flow passes a fixed sphere in an unbounded fluid domain . . 98

5.5 Initial configurations of two fibers . . . . . . . . . . . . . . 100

5.6 Fiber orientations of two far-away ellipsoidal fibers . . . . . . . . . 101

5.7 Fiber orientations of two close vertical ellipsoidal fibers . . . . . . . . 103

5.8 Fiber translations of two close vertical ellipsoidal fibers . . . . . . . . 104

5.9 Disturbance of fiber motion on fluid velocity, pressure and shear rate around fiber surface . . . . . . . . . . . . . . . 105

5.10 Fiber orientations of one vertical fiber and one horizontal fiber . . . . 106

5.11 Fiber translations of one vertical fiber and one horizontal fiber . . . . 107

5.12 Fiber orientations of two long ellipsoidal fibers . . . . . . . . . . . . 108

5.13 Fiber translations of two long ellipsoidal fibers . . . . . . . . . . . . . 109

5.14 Effect of various $\varepsilon^{\prime} s$ on fiber orientations . . . . . . . . . . . . 111

5.15 Plot of torques with different fiber orientation using various fluid domain.112

5.16 Fiber orientations in the bounded fluid domain. . . . . . . . . . . . . 113

5.17 Relationship between equivalent $\lambda$ and $\varepsilon$ for an ellipsoidal fiber with

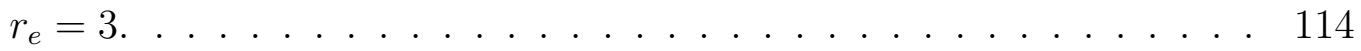

5.18 Relationship between equivalent $\lambda$ and $\varepsilon$ for a cylindrical fiber with $r_{e}=10 \ldots \ldots \ldots \ldots \ldots \ldots \ldots$

5.19 Definition of the effect of a stationary wall. . . . . . . . . . . . . 116

5.20 Fiber motion near a stationary wall . . . . . . . . . . . . . . . . 117

5.21 Relationship between time period of fiber rotation and parameter $\Delta d \quad 118$

A.1 Tetrahedral element used in the finite element model . . . . . . . . . 130 


\begin{abstract}
Short-fiber reinforced polymer composites enjoy widespread industrial applications due to their high strength-to-weight ratios and versatile manufacturing processes. The mechanical, electrical and thermal properties of short-fiber reinforced composite systems are tremendously dependent on fiber orientations within the polymer matrix during the manufacturing process. However, the commonly used melt flow simulation tools employ simplified empirically-derived models that have recently been shown to over-predict the rate of fiber alignment. Therefore, a physical understanding of fiber suspensions during the injection molding process is critical.

The main objective of this research project is to develop a systematic methodology to predict fiber orientations during the manufacture of polymer composites through the numerical simulation. The focus is to address such issues as the effect of fiber shape, fiber-fiber interactions, Brownian motions of nano-fibers and fiber suspensions in various solvents, such as inhomogeneous flows. We develop a stand-alone Finite Element Method (FEM) for calculating hydrodynamic forces and torques exerted on fibers. For nano-fibers, the Brownian forces and torques are modeled using a Gaussian distribution function. Our approach seeks fibers' velocities that zero the net torques and forces acting on the fibers by the surrounding bulk fluid. Fiber motions are then computed using a $4^{\text {th }}$-order Runge-Kutta method to update fiber positions and orientations as functions of time.

The successful completion of this project provides a systematic computational approach capable of addressing issues that are currently unresolved in the critical area of manufacturing. Extension of the approach to other areas such as drug delivery and blood cell motion is an additional benefit of this research work.
\end{abstract}




\section{Chapter 1}

\section{Introduction}

\subsection{Background}

Short-fiber reinforced polymer composite materials, well-known for their high strengthto-weight ratios, are widely used in various industries, such as aerospace, automotive and medical fields. Short-fiber polymer composite products are widespread since they can be manufactured into complicated shapes with improved properties obtained by adding up to $50 \%$ carbon/glass micro- or nano-size fibers into the molten polymer matrix. Short-fiber reinforced composites are always produced using the injection molding process (http://www.rutlandplastics.co.uk/moulding_process.shtml). As seen in Fig.1.1, discrete fibers and resin are mixed in the barrel and conveyed forward with the help of a feedings screw, which generates huge heat and pressure. After the mixture is forced into the mold cavity with high pressure and temperature, the resin exhibits the Newtonian or non-Newtonian properties, and discrete fibers translate and rotate within the resin. After the mixture is solidified using the cooling or curing treatment, the final molded part is produced.

Mechanical, electrical and thermal properties of short-fiber reinforced composite 


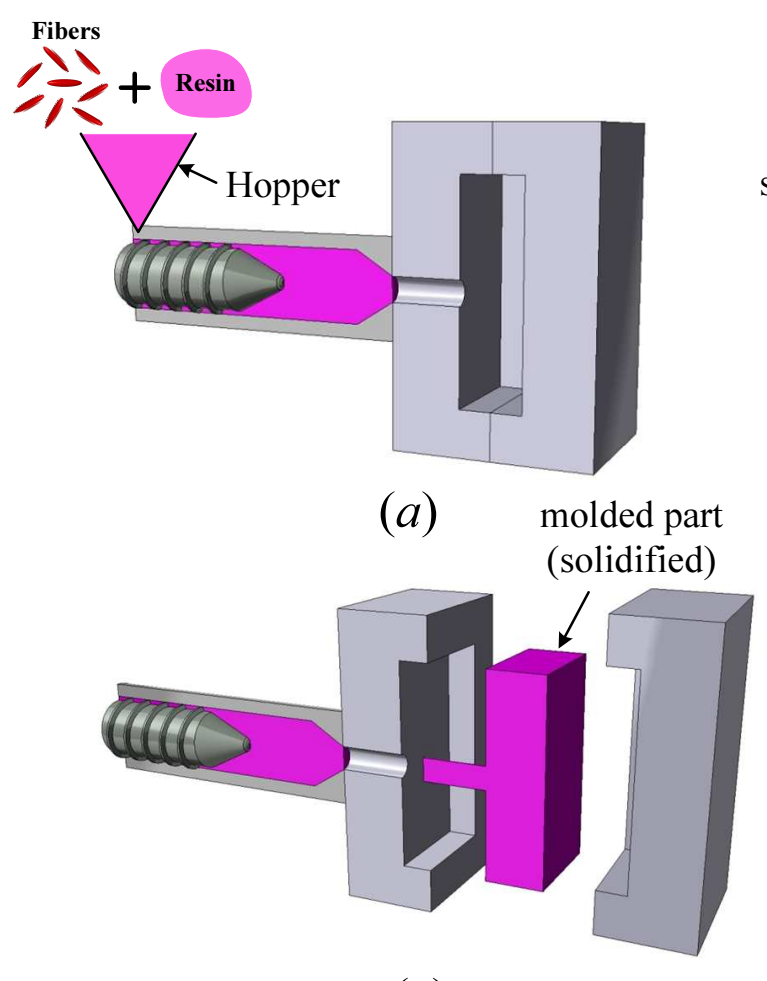

(c)

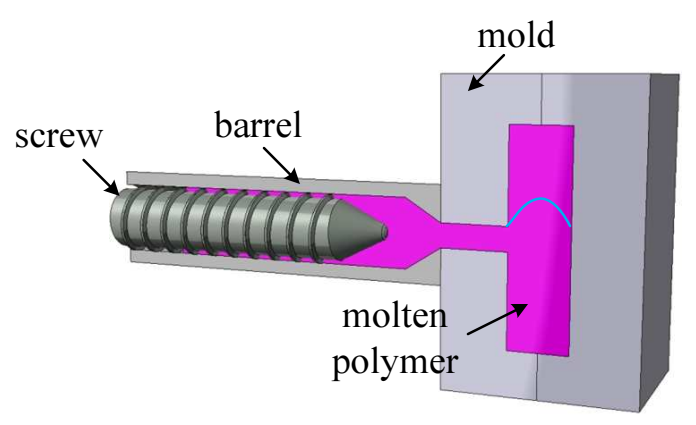

(b)

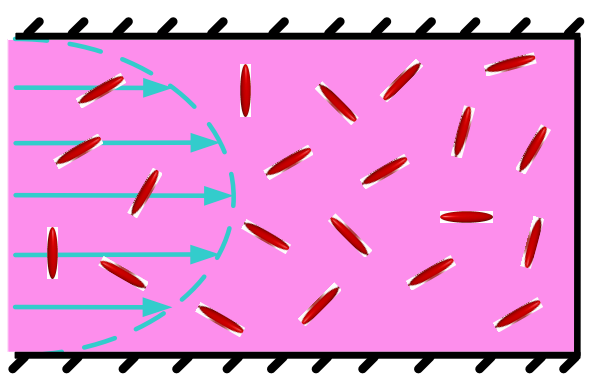

(d)

Figure 1.1: Injection molding process: (a) Mixture of fibers and resin in a heated barrel with a rotating screw; $(b)$ Forming of molten polymer into the mold cavity; (c) Solidification of molded part; $(d)$ Fiber suspensions in the molten polymer matrix during the manufacturing process.

systems are largely dependent on the fiber orientations within the polymer matrix [1-5], which are determined during the injection molding process. The good understanding of fiber orientations helps to control fiber motions so as to achieve the improved products [6]. One of the first publications on the movement of a particle within a viscous fluid is Einstein's paper [7], dealing with a spherical particle. The orientation state of an ellipsoidal fiber is often computed based on the method first proposed by Jeffery [8], who studied a single ellipsoidal fiber rotation in a Newtonian, incompressible, simple shear flow. In Jeffery's theory, fiber translates with the same translational velocity as the undisturbed bulk flow evaluated at fiber centroid. Fiber orientation is defined by three angles $(\phi, \theta, \psi)$ in terms of the $x y z$ coordinate system, as shown in Fig.1.2. The angles $\phi$ and $\theta$ are used to define the unit direction of the 
primary axis, i.e. the long axis of an ellipsoid, denoted as $\mathbf{P}$, and $\psi$ represents the fiber rotation along the unit direction vector $\mathbf{P}$, defined as

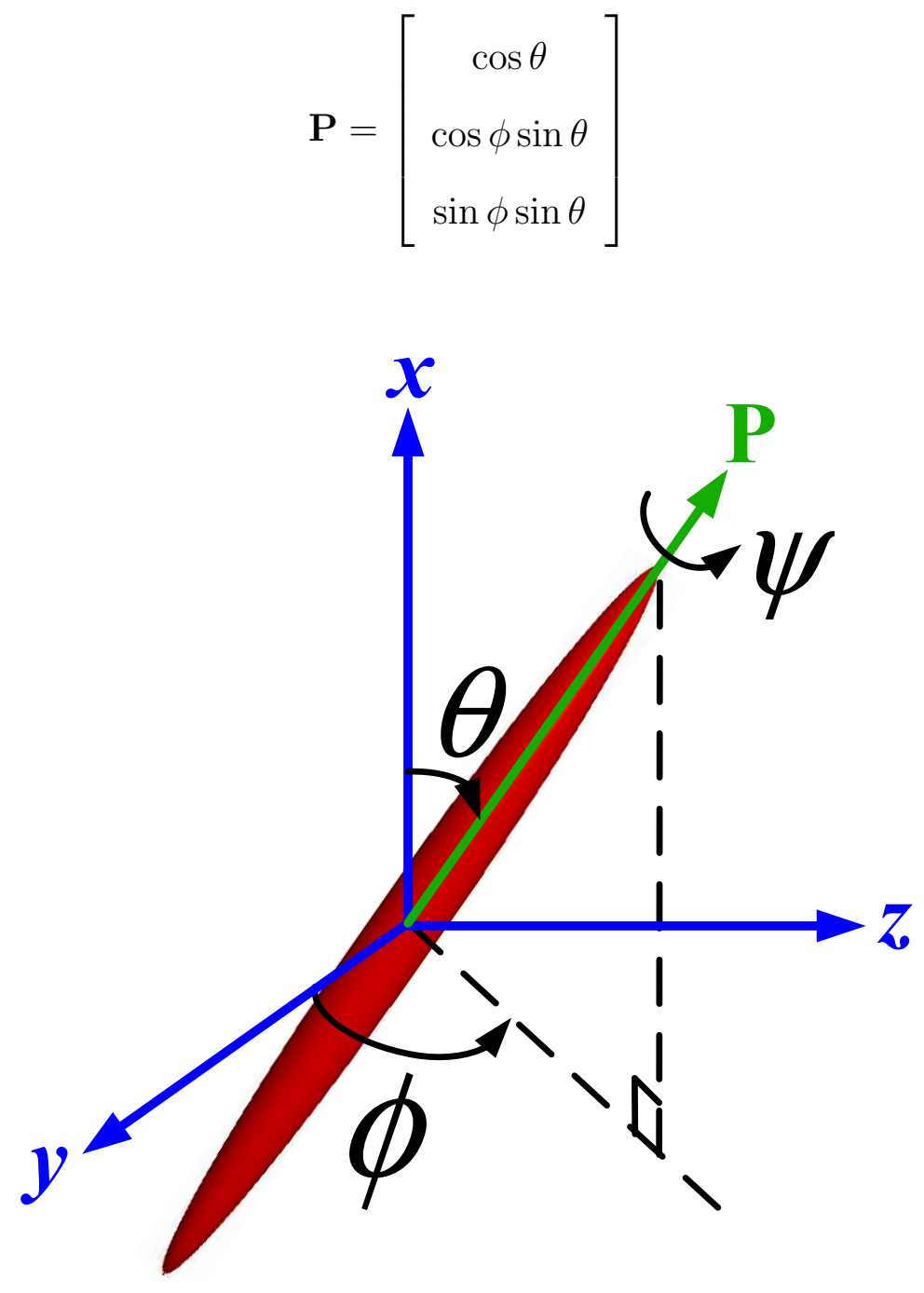

Figure 1.2: Fiber orientation of an ellipsoidal fiber defined in Jeffery's theory Applying Lamb's classic treatise [9] and assuming a torque-free particle, Jeffery solved $\phi(t), \theta(t), \psi(t)$ for the fiber orientation within a simple shear flow with $\mathbf{U}=$ 
$\left[U_{x}, U_{y}, U_{z}\right]^{T}=[0,0, \dot{\gamma} y]^{T}$, as seen in Eq.(1.2).

$$
\begin{aligned}
& \phi(t)=\tan ^{-1}\left(r_{e} \tan \frac{\dot{\gamma} t}{r_{e}+1 / r_{e}}\right) \\
& \theta(t)=\tan ^{-1} \frac{C r_{e}}{\sqrt{r_{e}^{2} \cos ^{2} \phi+\sin ^{2} \phi}} \\
& \psi(t)=\int_{0}^{t}\left(\frac{\dot{\gamma}}{2}-\dot{\phi}\right) \cos \theta d t
\end{aligned}
$$

where $\dot{\gamma}$ is the scalar magnitude of the strain rate tensor $\Gamma$, defined as

$$
\dot{\gamma}=\sqrt{\frac{1}{2} \Gamma: \Gamma}
$$

where

$$
\Gamma=\frac{1}{2}\left[\nabla \mathbf{U}+(\nabla \mathbf{U})^{T}\right]
$$

In Eq.(1.2), $r_{e}$ is the ratio of the long axis to the short axis of an ellipsoid, often referred to as geometric aspect ratio. The "orbit constant" $C$ is determined by the initial fiber orientation, and varies between $C=0$ corresponding to the fiber rolling along its symmetric axis, and $C=+\infty$ representing the periodic tumbling motion of the fiber in the $y z$ plane, as shown in Fig.1.3. By differentiating Eq.(1.2) with respect to time, the first-order derivatives of angles with respect to time, $\dot{\phi}(t), \dot{\theta}(t)$ and $\dot{\psi}(t)$, are obtained. In Jeffery's theory, a single fiber performs a periodic tumbling motion, with the time period defined as

$$
T=\frac{2 \pi}{\dot{\gamma}}\left(r_{e}+\frac{1}{r_{e}}\right)
$$

which is simply a function of the flow shear rate $\dot{\gamma}$ and the ellipsoidal fiber's geometric aspect ratio $r_{e}$. Hence, fiber shape has a great influence on the fiber motion, which is further discussed in Chapter 2.

Jeffery's theory was later extended to describe a single axisymmetric fiber orienta- 


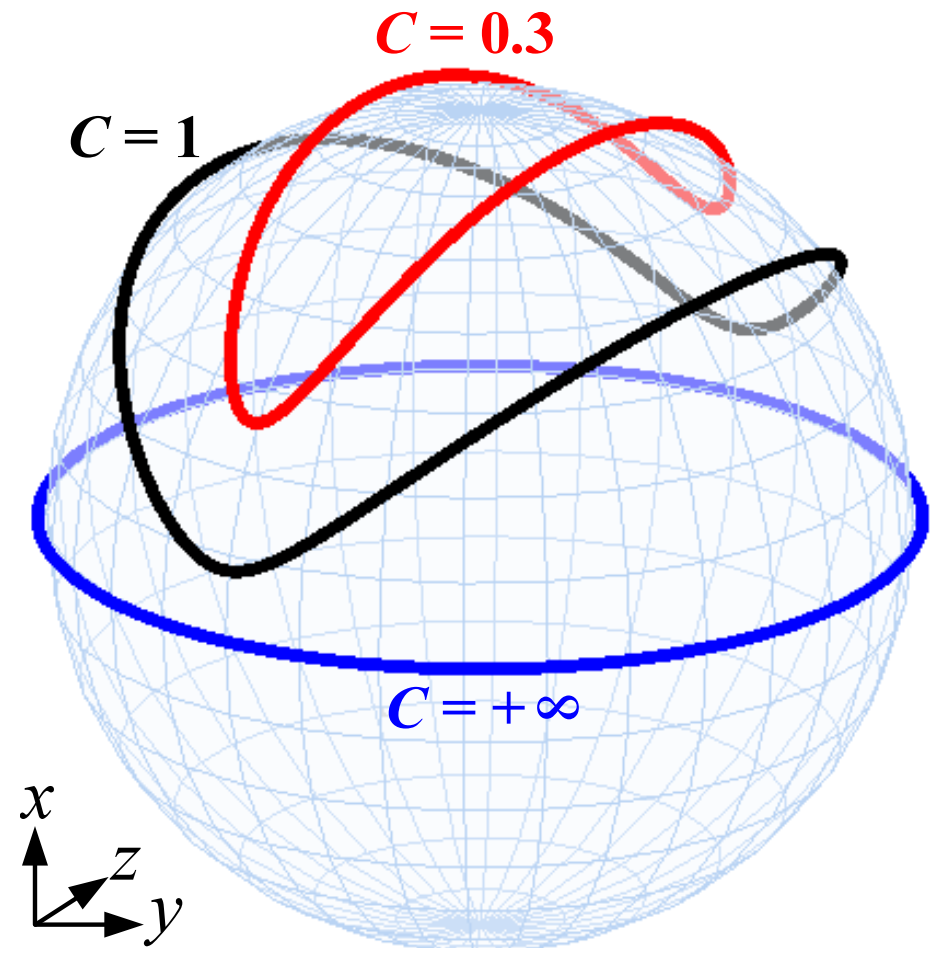

Figure 1.3: Jeffery's orbit with different orbit constants $C^{\prime} s$

tion in any homogeneous flows, i.e. velocity gradient of the undisturbed flow fluid is independent of spatial coordinate. The unit direction vector $\mathbf{P}$ is obtained by solving the following differential equation $[10,11]$ as

$$
\frac{d \mathbf{P}}{d t}=\Omega \mathbf{P}+\lambda\left[\Gamma \mathbf{P}-\left(\mathbf{P}^{T} \Gamma \mathbf{P}\right) \mathbf{P}\right]
$$

where $\lambda$ is related to fiber's geometry, calculated as

$$
\lambda=\frac{r_{e}^{2}-1}{r_{e}^{2}+1}
$$

In Eq.(1.6), $\Omega$ is the vorticity tensor of the undisturbed flow $\mathbf{U}$, defined as

$$
\Omega=\frac{1}{2}\left[\nabla \mathbf{U}-(\nabla \mathbf{U})^{T}\right]
$$


It can be seen that Jeffery's equation only describes the orientation of a single fiber. But in short-fiber reinforced polymer composite systems, there exist a large quantity of fibers in the polymer matrix. Therefore, it is critical to have a understanding of the orientation states of multiple fibers for the semi-dilute or concentrated fiber suspensions. Folgar and Tucker III [10] adopted a statistic approach to predict the probability distribution function $\chi(\mathbf{P})$ of fiber orientation. This probability distribution function describes the probability of fibers pointing at the unit direction $\mathbf{P}$, denoted as $\chi(\mathbf{P}) d \mathbf{P}$. There are three physical conditions associated with the distribution function [10], summarized as

1) The fiber in the direction $\mathbf{P}$ is indistinguishable from the fiber pointing at the direction $-\mathbf{P}$, i.e.

$$
\chi(\mathbf{P})=\chi(-\mathbf{P})
$$

2) The probability of fiber orientations in all directions must meet the following condition,

$$
\oint \chi(\mathbf{P}) d \mathbf{P}=1
$$

3) The continuity condition can be written as

$$
\frac{D \chi}{D t}=-\frac{\partial}{\partial \mathbf{P}} \cdot(\chi \dot{\mathbf{P}})
$$

where $D / D t(=\partial / \partial t+\mathbf{U} \cdot \nabla)$ represents the material derivative. If the expression of $\dot{\mathbf{P}}$ is known, the governing equation for $\chi$ could be obtained. Equation (1.6) is only applicable to the dilute system, in which each fiber moves freely, without the consideration of fiber-fiber interactions. Bur for the semi-dilute and concentrate fiber suspensions, fiber-fiber interactions cannot be neglected. So Eq.(1.6) is not a good candidate for that purpose. By observing the fiber behavior in concentrated suspensions, Folgar and Tucker III [10] suggested to add a diffusion term $D_{r}$ to $\dot{\phi}$ assuming a planar fiber motion, i.e. only $\phi$ changes with time, while $\theta$ and $\psi$ remain unchanged. 
This diffusion term is used to account for the effect of fiber-fiber interactions on fiber motions. Then the diffusion term was added to Eq.(1.6) by Advani and Tucker III [12], with the modified differential equation governing $\mathbf{P}$ shown as follows,

$$
\frac{D \mathbf{P}}{D t}=\Omega \mathbf{P}+\lambda\left[\Gamma \mathbf{P}-\left(\mathbf{P}^{T} \Gamma \mathbf{P}\right) \mathbf{P}\right]-D_{r} \frac{1}{\chi} \frac{\partial \chi}{\partial \mathbf{P}}
$$

where the diffusion term $D_{r}$ is defined as

$$
D_{r}=C_{I} \dot{\gamma}
$$

where $C_{I}$ is a phenomenological parameter modeling the randomizing effect of fiberfiber interactions, and is fitted by the experimental data.

Orientation averaging using the modified Jeffery's equation with the diffusion term (cf.Eq.(1.12)) has since yielded the governing equation for distribution function [10] and orientation tensor [12]. By plugging Eq.(1.12) into Eq.(1.11), the evolution of $\chi$ with respect to time can be obtained, which gives the statistical point of view of fiber orientation as a function of time. The governing equation for the distribution function $\chi$ can be seen in Refs. $[12,13]$. This is a second-order partial differential equation, which is difficulty to solve. The recent spherical harmonic solution approach [14] for solving Jeffery's equation provides a computationally efficient means to evaluate the computationally prohibitive orientation distribution function, but not directly applicable to industrial software solution approaches. In order to avoid the complexity of solving the distribution function, Advani and Tucker III [12] proposed to use orientation tensor instead of distribution function to describe the orientation states of fiber suspensions, which is applied regularly in industrial mold filling simulations. The 
second- and fourth-order orientation tensors are formed as

$$
\begin{aligned}
& \mathbf{A}=\oint \mathbf{P} \mathbf{P} \chi(\mathbf{P}) d \mathbf{P} \quad \text { or } \quad A_{i j}=\oint p_{i} p_{j} \chi(\mathbf{P}) d \mathbf{P} \\
& \mathbb{A}=\oint \mathbf{P P P P} \chi(\mathbf{P}) d \mathbf{P} \quad \text { or } \quad A_{i j k l}=\oint p_{i} p_{j} p_{k} p_{l} \chi(\mathbf{P}) d \mathbf{P}
\end{aligned}
$$

By plugging Eq.(1.14) into the evolution of $\chi$, the evolution of the second-order orientation tensor can be represented as

$$
\frac{D \mathbf{A}}{D t}=\Omega \mathbf{A}-\mathbf{A} \Omega+\lambda(\Gamma \mathbf{A}+\mathbf{A} \Gamma-2 \mathbb{A}: \Gamma)+2 D_{r}(\mathbf{I}-3 \mathbf{A})
$$

where $\mathbf{I}$ is the identity matrix. To solve Eq.(1.16) to get the second-order orientation tensor $\mathbf{A}$, the fourth-order orientation tensor $\mathbb{A}$ is needed in advance. So there exists a "closure" problem. The common solution to this problem is to use closure approximation, a method to approximate $\mathbb{A}$ using $\mathbf{A}$. Many closure approximations, such as quadratic closure [15], hybrid closure [12], eigenvalue-based closure [13], natural closure $[16,17]$ and an invariant based fitted closure [18], have been developed to allow orientation tensor solutions within industrial mold filling software packages (see e.g., Moldflow, Autodesk, Inc., Farmingham, MA, www.moldflow.com) and quickly provide reasonably accurate results.

Many researchers have extended Jeffery's work to study fiber suspensions in broader areas [19-22]. Jia [23] considered a slip boundary condition on the fiber surface, while Jeffery applied the non-slip boundary condition. Junk [11] theoretically studied the effect of a bounded wall around the fiber, and found that Jeffery's result represents the leading order equation of a singularly perturbed flow problem. Leal [20] analyzed the non-Newtonian effect of a fluid with the presence of fibers, and studied fiber motions under the consideration of weak fluid inertia. To study non-dilute fiber suspensions, Batchelor $[24,25]$ proposed a constitutive model to take 
into account the contributions that fibers have to the fluid's total stress tensor. Sus-

pensions of flexible fibers [26-29] have also been simulated. Yamamoto [26] regarded a fiber as a group of beads that are lined up and bonded to each neighbor as a bead chain, referred to as a bead-chain fiber, where each pair of bonded spheres can stretch, bend and twist. Ross and Klingberg [27] and Wang et al. [28] used a rod-chain model to represent flexible fibers in a similar manner.

\subsection{Research motivation}

Jeffery's theory serves as the basis for describing fiber orientations in short-fiber reinforced composite materials. Jeffery showed that a single fiber has a closed periodic tumbling motion in a simple shear flow. Even though this periodic motion has been validated in experiments for dilute suspensions [30-33], the observed period of fiber motion is slower than the direct implementation of Jeffery's equation [30]. In addition, the orientation states of concentrated fiber suspensions obtained by evaluating orientation tensors show a deviation from Jeffery's orbit $[34,35]$, which may result from the limiting assumptions in Jeffery's original work. The plot of one component of orientation tensor is cited from Tucker III's report [35], as seen in Fig.1.4, from which it can be seen that the experimental data deviates from Jeffery-based standard theory.

Furthermore, the diffusion term $D_{r}$ includes the phenomenological coefficient $C_{I}$, which is fitted using the experimental data. Currently, there is no general rule to guide the choice of $C_{I}$ for different flow conditions. Some research work has been done to point out the shortcomings of Folgar and Tucker III's diffusion model [36, 37]. The physical understanding of flow-induced fiber suspensions are still in question. Therefore, fiber motions in complicated flows motivate us to physically model discrete fiber motions in various flow situations and explore the possible factors that affect 

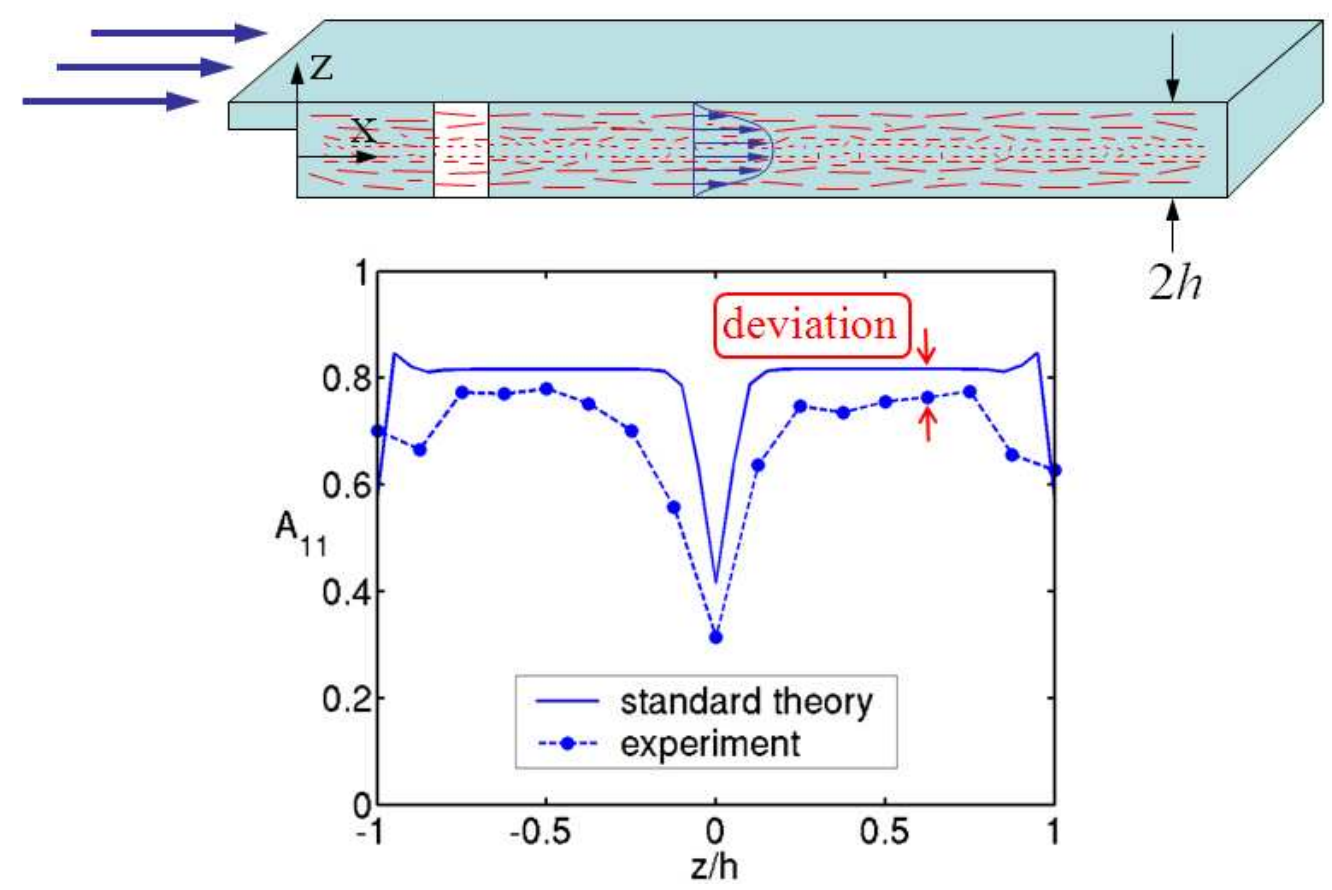

$2 h$

Figure 1.4: Deviation of orientation tensor in experimental data from theory

fiber motions.

\subsection{Thesis objectives}

The main objectives of this dissertation are to 1) physically understand discrete fiber suspensions within the polymer matrix and 2) investigate complicated issues on the prediction of fiber orientations in short-fiber reinforced composites, which are not addressed in Jeffery's theory or not well understood in current research community. These objectives are realized with the following research:

- Develop a stand-alone FEM-based simulation tool to predict fiber motions during the mold filling process.

- Verify the proposed methodology with the current theories, such as Jeffery's theory and experimental data.

- Address such issues as the role of fiber shape, the effect of fluid domain, fiber suspensions in inhomogeneous solvents, Brownian motions of nano-fibers and 
the effect of fiber-fiber interactions.

The proposed computational methodology is used to gain unprecedented insight into how to control fiber orientations in micro- and nano-polymer composite suspensions in order to obtain the best engineered products.

\subsection{Organization of the thesis}

The reminder of this dissertation is organized as follows:

Chapter 2 presents a computational approach for simulating the motion of a single fiber suspended within a viscous fluid using a commercial FEM solver (COMSOL Multiphysics). Our approach seeks solutions using the Newton-Raphson method for fiber's linear and angular velocities such that the net hydrodynamic force and torque acting on the fiber are zero. Fiber motion is then computed with a Runge-Kutta method to update fiber position and orientation as functions of time. This approach is first used to verify Jeffery's orbit [8] and addresses such issues as the role of fiber shape on the dynamics of a single fiber, which were not considered in Jeffery's original work. The method is quite general and allows for fiber shapes that include, but are not limited to, ellipsoidal fibers (such as those studied in Jeffery's original work), cylindrical fibers, and bead-chain fibers. The relationships between equivalent aspect ratios and geometric aspect ratios of cylindrical and other axisymmetric fibers are derived in this chapter.

Chapter 3 presents a stand-alone three-dimensional finite element simulation tool to investigate the fiber motion in unbounded Newtonian flows, including homogeneous flows (such as rotational and extensional flows) and inhomogeneous flows (such as Poiseuille flows). Instead of using commercial FEM solver, we propose a self-made FEM package coupled with the analytical Jacobian matrix in the Newton-Raphson iteration and transformed essential boundary conditions without the re-meshing pro- 
cess, which achieves forty times better computation efficiency over that using the commercial solver proposed in Chapter 2. The three-dimensional/full fiber motions in various low-Reynolds-number flows are tracked and compared with current theories and experimental work. Results confirm that the Segré-Silberberg phenomenon does not happen in creeping Poiseuille flows. Fiber orientation is still governed by Jeffery's theory, but fiber's linear motion is retarded, i.e. fiber lags behind the undisturbed flow with the velocity evaluated at fiber centroid.

Chapter 4 extends the methodology proposed in Chapter 3 to simulate the motions of nano-fibers during the nano-polymer composites processing. A Langevin approach is used to account for both hydrodynamic and Brownian effects. The hydrodynamic force and torque exerted from the surrounding fluid are modeled using the FEM package presented in Chapter 3, and the Brownian force and torque are regarded as the random thermal disturbing effects, which are modeled using a Gaussian process. Since Brownian disturbance from the surrounding fluid molecules is a stochastic process, Monte-Carlo simulation is used to evaluate the motions of a large quantity of fibers associated with different random Brownian forces and torques. The final fiber motion is obtained by averaging a numerous fiber motion paths. Examples of fiber motions with various Péclet numbers are presented in this chapter.

Chapter 5 investigates semi-dilute fiber suspensions in a bounded fluid domain using the methodology proposed in Chapter 3. For industrial applications, the volume fraction of short fibers is large for semi-dilute and concentrated fiber suspensions. Therefore, fiber-fiber interactions affect fiber orientations and thus the rate of fiber alignment. In this chapter, we study the semi-dilute fiber suspensions, i.e. the gap between fibers becomes closer, and hydrodynamic interactions becomes stronger, but the physical/mechanical contacts are still rare. Compared with the fiber size, the fluid domain of the polymer matrix is relatively large, so the unbounded assumption is valid for a single fiber motion. But the existence of many other fibers around 
one fiber reduces the fluid domain, so the study of fiber motion in a bounded fluid domain is critical in investigating the effect of semi-dilute or concentrated suspensions. Meanwhile, during the injection molding process, some fibers move close to one side of the mold cavity, which changes the behavior of fibers. In this chapter, we physically address three critical issues in flow-induced fiber suspensions: fiber-fiber interaction between two fibers, the effect of bounded fluid domain and the fiber motion near a stationary wall.

The last chapter concludes our research work and provides the discussions on the future research direction. 


\section{Chapter 2}

\section{Numerical Evaluation of the Motion of Fibers with Various Shapes Using Commercial FEM Solver}

\section{$2.1 \quad$ Introduction}

Jeffery's theory serves as the basis for describing fiber orientations in short-fiber reinforced composite materials, and better understanding the underlying dependence of orientation distribution on fiber's geometry is worth investigating further. Only the ellipsoidal fiber was considered in Jeffery's theory. However, non-ellipsoidal shapes, such as cylindrical fibers, are of more interest in the area of composite materials processing. Therefore, the focus of this chapter is to evaluate the motion of cylindrical fibers as well as the fibers with other shapes.

One main factor that defines Jeffery's orbit is fiber's geometric aspect ratio, related to fiber's geometry. The study of the motions of fibers with different shapes was also studied by Bretherton [38], who proposed that Jeffery's theory is also valid for general 
axisymmetric bodies with fore-aft symmetry, when an equivalent aspect ratio $\left(r_{e}^{*}\right)$ is used, instead of the geometric aspect ratio $\left(r_{e}\right)$, to quantify the fiber orientation. For cylindrical fibers, the periodic tumbling motion is observed in Refs.[31, 33, 39, 40], but cylindrical fibers have different observed rotation periods from those of ellipsoidal fibers. Trevelyan [31] experimentally estimated the ratio of the equivalent aspect ratio to the geometric aspect ratio $\left(r_{e}^{*} / r_{e}\right)$ to be approximately 0.7 . Petrich [39] found that the ratio is 0.687 for $r_{e}=50$ and 0.623 for $r_{e}=72$. Cox [41] proposed a theoretical formula to calculate the equivalent aspect ratio of a slender cylindrical fiber. Unfortunately, his equation is not valid for short rod-like fibers with aspect ratios smaller than 20. Additionally, we note that existing simulation methods have given little attention to other fiber shapes.

In this chapter, we present an approach that addresses the general three-dimensional motions of axisymmetric fibers with various geometries. Specifically, a Finite Element Method is applied to solve the governing equations of fluid flow, so as to evaluate the hydrodynamic force and torque. The Newton-Raphson method is used to search for fiber's linear and angular velocities which zero the hydrodynamic force and torque on the fiber. The Runge-Kutta method updates fiber position and orientation with respect to time. To ensure the accuracy and robustness of our methodology, the results from our approach are compared with Jeffery's theory using an ellipsoidal fiber. In this chapter, our focus is to develop a tool to simulate the motion of a single fiber suspended within a viscous fluid.

The remainder of this chapter is organized as follows: Section 2.2 presents the methodology, including the Finite Element Method, the Newton-Raphson iteration method and the Runge-Kutta method. Section 2.3 presents the computed equivalent aspect ratios of axisymmetric fibers, including ellipsoidal, cylindrical and bead-chain fibers. Implementation and illustrative examples are given in Sec.2.4, and summary is presented in Sec.2.5. 


\subsection{Methodology}

In this chapter, we apply numerical schemes to solve for the motion of a single fiber within a viscous fluid. The basis is that the fiber is migrating with zero net force and torque from the fluid. As in Jeffery's approach, we also use $\phi, \theta, \psi$ to define the fiber orientation, as seen in Fig.1.2. The position vector of fiber centroid in the $x y z$ system is defined as $\left[x_{c}, y_{c}, z_{c}\right]^{T}$, and has a translation velocity $\left[\dot{x}_{c}, \dot{y}_{c}, \dot{z}_{c}\right]^{T}$.

\subsubsection{Fluid dynamics simulation}

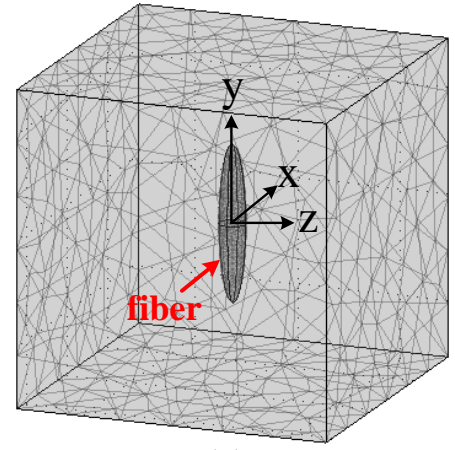

(a)

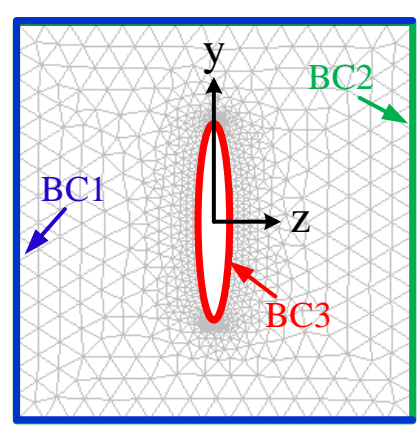

(b)

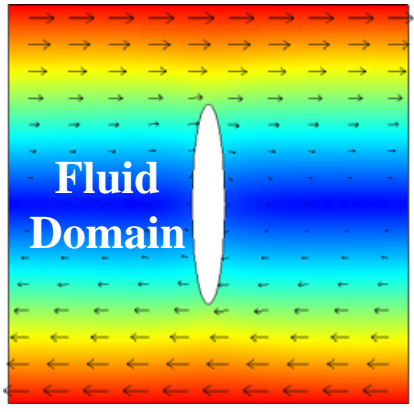

(c)

Figure 2.1: (a) Three-dimensional finite element model; (b) Mesh model in the yz plane with three boundary conditions; (c) Velocity distribution of fluid domain

In the proposed approach, the Finite Element Method is used to evaluate the velocity and pressure distribution within the fluid to calculate the force and torque on a fiber. The governing equations and boundary conditions are defined in the coordinate system $x y z$, where the continuity equation and the steady Navier-Stokes equation are given respectively as

$$
\begin{gathered}
\nabla \cdot \mathbf{U}=0 \\
\rho \mathbf{U} \cdot \nabla \mathbf{U}=-\nabla p+\mu \nabla^{2} \mathbf{U}
\end{gathered}
$$

where $\mathbf{U}$ and $p$ are, respectively, the velocity and pressure of the fluid, $\rho$ is the density 
of the fluid, and $\mu$ is the absolute viscosity of the fluid. We use tetrahedral meshes to represent the three-dimensional fluid domain shown in Fig.2.1(a), with the three boundary conditions shown in Fig.2.1(b): the inlet fluid velocity (BC1), the outlet pressure (BC2) and the fluid velocity on fiber surface (BC3). The three boundary conditions in COMSOL are defined as

(BC1) Inlet velocity of far-away fluid boundary:

$$
\mathrm{U}_{\mathrm{BC} 1}=\mathbf{U}_{0}
$$

(BC2) Outlet pressure of far-away fluid boundary:

$$
p_{\mathrm{BC} 2}=p_{0}
$$

(BC3) Moving wall on fiber surface:

$$
\mathrm{U}_{\mathrm{BC} 3}=\mathrm{U}_{c}+\boldsymbol{\omega} \times \mathbf{r}
$$

where $\mathbf{U}_{c}=\left[\dot{x}_{c}, \dot{y}_{c}, \dot{z}_{c}\right]^{T}$ is the translational velocity of the fiber centroid and $\mathbf{r}$ stands for the position vector of the nodes on fiber surface with respect to fiber centroid. On $\mathrm{BC} 1, \mathrm{U}_{0}$ is the undisturbed simple shear flow, and on $\mathrm{BC} 2, p_{0}$ is the benchmark pressure, usually set up to be zero. Fiber's angular velocity $\boldsymbol{\omega}$ is defined as [11]

$$
\boldsymbol{\omega}=\left[\begin{array}{c}
\omega_{x} \\
\omega_{y} \\
\omega_{z}
\end{array}\right]=\left[\begin{array}{c}
\dot{\phi}+\dot{\psi} \cos \theta \\
-\dot{\theta} \sin \phi+\dot{\psi} \sin \theta \cos \phi \\
\dot{\theta} \cos \phi+\dot{\psi} \sin \theta \sin \phi
\end{array}\right]
$$

We solve Eqs.(2.1)-(2.5) with the Finite Element Method to obtain the net force 
$\mathbf{F}$ and net torque $\mathbf{T}$ on the fiber computed from the following equations

$$
\begin{aligned}
\boldsymbol{\sigma} & =-p \boldsymbol{\delta}+\mu\left[\nabla \mathbf{U}+(\nabla \mathbf{U})^{T}\right] \\
\mathbf{F} & =\int\{\boldsymbol{\sigma} \cdot \mathbf{n}\} d S \\
\mathbf{T} & =\int\{\mathbf{r} \times(\boldsymbol{\sigma} \cdot \mathbf{n})\} d S
\end{aligned}
$$

where $\boldsymbol{\sigma}$ is the stress in fluid, $\boldsymbol{\delta}$ is the Kronecker delta, $d S$ represents the surface integral, $\mathbf{n}$ is the unit normal vector on fiber surface, and $\times$ represents the cross product operator.

\subsubsection{Computing fiber velocities}

In the finite element model, $\mathbf{U}_{c}$ and $\boldsymbol{\omega}$ define the boundary condition BC3 (cf. Eq.(2.5) when solving for $\mathbf{U}$ and $p$ within the fluid, and also the force $\mathbf{F}$ and torque $\mathbf{T}$ from Eqs.(2.8) and (2.9), respectively. Therefore, $\mathbf{F}$ and $\mathbf{T}$ are functions of $\dot{x}_{c}, \dot{y}_{c}, \dot{z}_{c}$ and $\dot{\phi}, \dot{\theta}, \dot{\psi}$. The force and torque exerted on the massless fiber are set to zero (as in Jeffery's original work) at any time instant $t_{i}$ as

$$
\begin{aligned}
& \mathbf{F}\left(\dot{x}_{c i}, \dot{y}_{c i}, \dot{z}_{c i}, \dot{\phi}_{i}, \dot{\theta}_{i}, \dot{\psi}_{i}\right)=\mathbf{0} \\
& \mathbf{T}\left(\dot{x}_{c i}, \dot{y}_{c i}, \dot{z}_{c i}, \dot{\phi}_{i}, \dot{\theta}_{i}, \dot{\psi}_{i}\right)=\mathbf{0}
\end{aligned}
$$

using the Newton-Raphson iteration algorithm to compute fiber's linear and angular velocities at any time $t_{i}$. This is done by solving Eqs.(2.10) and (2.11) simultaneously as

$$
\dot{\mathbf{y}}_{I+1}=\dot{\mathbf{y}}_{I}-\left[\mathbf{J}_{I}\right]^{-1}\left[\mathbf{Q}_{I}\right]
$$

where $I$ is the Newton-Raphson iteration number, $\dot{\mathbf{y}}_{I}=\left[\dot{x}_{c i}, \dot{y}_{c i}, \dot{z}_{c i}, \dot{\phi}_{i}, \dot{\theta}_{i}, \dot{\psi}_{i}\right]^{T}$, and 
$\left[\mathbf{Q}_{I}\right]$ is a function of $\mathbf{F}$ and $\mathbf{T}$, given as

$$
\left[\mathbf{Q}_{I}\right]=\left[F_{x}, F_{y}, F_{z}, T_{x}, T_{y}, T_{z}\right]^{T}
$$

In our analysis, $\left[\mathbf{J}_{I}\right]$ is the Jacobian matrix (i.e., the first order partial derivatives of $\left[\mathbf{Q}_{I}\right]$ with respect to $\dot{\mathbf{y}}_{I}$ ), which is evaluated using the forward finite difference method.

\subsubsection{Evolution of fiber motion}

At any time instant $t_{i}$, we assume that fiber's centroid $x_{c i}, y_{c i}, z_{c i}$ and fiber orientation $\phi_{i}, \theta_{i}, \psi_{i}$ are given, which define the configuration of the fiber within the finite element simulation. The linear velocity $\left(\dot{x}_{c i}, \dot{y}_{c i}, \dot{z}_{c i}\right)$ and angular velocity (defined by $\dot{\phi}_{i}, \dot{\theta}_{i}, \dot{\psi}_{i}$ ) are then obtained from the converged Newton-Raphson iteration to give $\dot{\mathbf{y}}_{i}=\mathbf{f}\left(t_{i}, \mathbf{y}_{i}\right)$, where $\mathbf{y}_{i}=\left[x_{c i}, y_{c i}, z_{c i}, \phi_{i}, \theta_{i}, \psi_{i}\right]^{T}$. We use a $4^{\text {th }}$-order Runge-Kutta algorithm to solve

the evolution of $x_{c}, y_{c}, z_{c}, \phi, \theta, \psi$ as a function of time, with the given initial conditions $\left[x_{c 0}, y_{c 0}, z_{c 0}, \phi_{0}, \theta_{0}, \psi_{0}\right]^{T}$. The algorithm is shown as follows [42]:

$$
\mathbf{y}_{i+1}=\mathbf{y}_{i}+\frac{1}{6} \Delta t\left(\mathbf{k}_{1}+2 \mathbf{k}_{2}+2 \mathbf{k}_{3}+\mathbf{k}_{4}\right)
$$

where

$$
\begin{aligned}
& \mathbf{k}_{1}=\mathbf{f}\left(t_{i}, \mathbf{y}_{i}\right) \\
& \mathbf{k}_{2}=\mathbf{f}\left(t_{i}+0.5 \Delta t, \mathbf{y}_{i}+0.5 \mathbf{k}_{1} \Delta t\right) \\
& \mathbf{k}_{3}=\mathbf{f}\left(t_{i}+0.5 \Delta t, \mathbf{y}_{i}+0.5 \mathbf{k}_{2} \Delta t\right) \\
& \mathbf{k}_{4}=\mathbf{f}\left(t_{i}+\Delta t, \mathbf{y}_{i}+\mathbf{k}_{3} \Delta t\right)
\end{aligned}
$$

In Eq.(2.14), $\Delta t$ represents the time interval in the Runge-Kutta method, and $\mathbf{y}_{i+1}$ 
is the value of $\mathbf{y}$ at time $t_{i+1}$.

\subsubsection{Summary of numerical approach}

Our numerical approach proceeds as follows:

STEP 1: At time $t_{i}(i$ starts from 0$)$, the finite element model of the fluid domain between the fiber and the far-away fluid boundary is defined. In this model, we use

$\dot{x}_{c i}, \dot{y}_{c i}, \dot{z}_{c i}$ and $\dot{\phi}_{i}, \dot{\theta}_{i}, \dot{\psi}_{i}$ to define the velocity boundary condition on the fiber surface so as to calculate the hydrodynamic force and torque exerted on fiber. Note that based on Jeffery's theory, the values of $\dot{x}_{c i}, \dot{y}_{c i}, \dot{z}_{c i}, \dot{\phi}_{i}, \dot{\theta}_{i}, \dot{\psi}_{i}$ give rise to zero net force and torque on the fiber.

STEP 2: A Newton-Raphson method is used to calculate the expected fiber velocities, which generate zero net force and torque at each time instant $t_{i}$. Note that at different time instants, the solutions for the velocities are different, because the finite element model, defined by $x_{c i}, y_{c i}, z_{c i}, \phi_{i}, \theta_{i}, \psi_{i}$, is changing with respect to time.

STEP 3: Since at any time instant $t_{i}$, the linear velocity $\left(\dot{x}_{c i}, \dot{y}_{c i}, \dot{z}_{c i}\right)$ of the fiber centroid and angular velocity (defined by $\left.\dot{\phi}_{i}, \dot{\theta}_{i}, \dot{\psi}_{i}\right)$ are obtained, a $4^{\text {th }}$-order RungeKutta algorithm is used to update fiber position and orientation as functions of time.

\subsection{Role of fiber shape}

The hydrodynamic force and torque in Eqs.(2.8) and (2.9) are evaluated as the surface integrals on the exterior of the fiber, thus fiber shape has a significant effect on the dynamics of fiber motion within a fluid. Jeffery considered an ellipsoid-shaped fiber, but the motion of a fiber with other shapes, such as a cylindrical fiber as would be fabricated from a chopped-fiber process, is of more interest in the field of composite materials processing. Therefore, in this section, we explore the motions of cylindrical fibers and fibers having other axisymmetric shapes. 
For any axisymmetric fiber, the geometric aspect ratio $r_{e}$ is typically defined by fiber's geometry. For example, $r_{e}$ of an ellipsoidal fiber is the ratio of the long axis to the short axis. Alternatively, $r_{e}$ of a cylindrical fiber is commonly defined as the ratio of fiber's length to its diameter. Bretherton [38] theoretically demonstrated that any axisymmetric fiber has a periodic tumbling motion, which is also governed by Jeffery's theory. However, a different aspect ratio, referred to as the equivalent aspect ratio $r_{e}^{*}$ is used to quantify the fiber motion instead of the geometric aspect ratio $r_{e}$. Cox [41] used the ellipsoidal fiber to derive a formula for the equivalent aspect ratios by considering a simple shear flow $\mathbf{U}=[0,0, \dot{\gamma} y]^{T}$ with an orbit constant $C=+\infty$, such that $\theta=\pi / 2, \psi=0$ and $\dot{\theta}=\dot{\psi}=0$ in Eq.(2.6). The resulting angular velocity of the suspended fiber in the $x y z$ coordinate system is $\boldsymbol{\omega}=[\dot{\phi}, 0,0]^{T}$, where $\dot{\phi}$ is obtained by differentiating Eq.(1.2) with respect to time as

$$
\omega_{x}=\dot{\phi}=\frac{\dot{\gamma}}{r_{e}^{2}+1}\left[1+\left(r_{e}^{2}-1\right) \cos ^{2} \phi\right]
$$

Cox considered the angular velocity $\omega_{x}$ along the $x$ axis when the ellipsoid's long axis is in the vertical and horizontal directions, as shown in Fig.2.2.

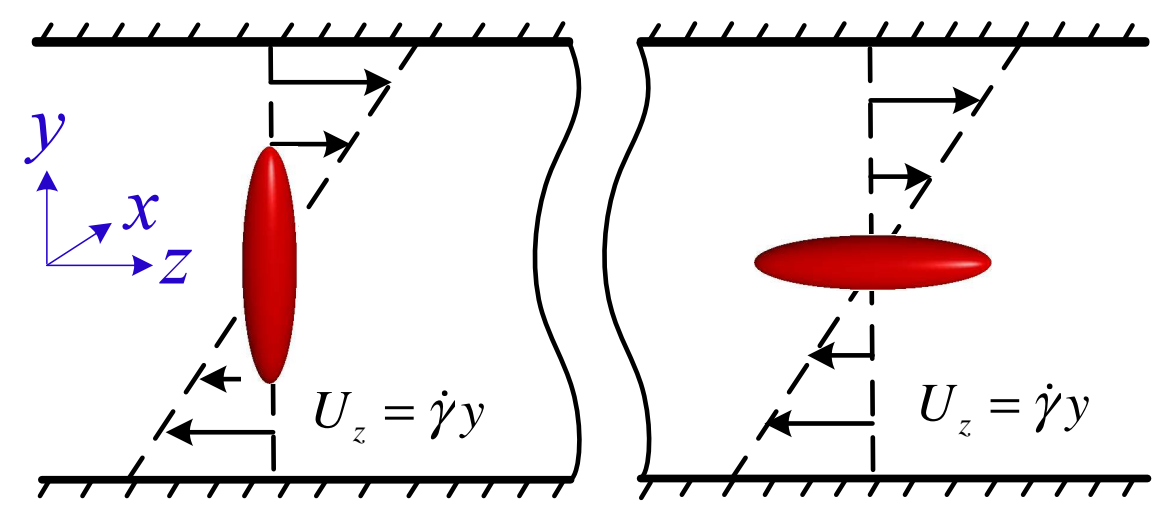

Figure 2.2: Torques on an ellipsoidal fiber fixed in two directions: (a) The vertical direction $(\phi=0)$ and $(b)$ the horizontal direction $(\phi=\pi / 2)$

In the vertical direction $(\phi=0)$, Eq.(2.15) yields $\left[\omega_{x}\right]_{V}=\frac{\dot{\gamma}}{r_{e}^{2}+1} r_{e}^{2}$, and similarly, in the horizontal direction $(\phi=\pi / 2),\left[\omega_{x}\right]_{H}=\frac{\dot{\gamma}}{r_{e}^{2}+1}$. Therefore, the equivalent aspect 
ratio of an ellipsoidal fiber is calculated as

$$
r_{e}^{*}=\sqrt{\frac{\left[\omega_{x}\right]_{V}}{\left[\omega_{x}\right]_{H}}}=r_{e}
$$

Note that for an ellipsoidal fiber, the equivalent aspect ratio equals its geometric aspect ratio. Cox [41] proposed that the torque $T_{x}$ exerted on fibers when the fiber is fixed should be proportional to fiber's angular velocity $\omega_{x}$ when it is free to rotate. Therefore, the equivalent aspect ratio of any axisymmetric fiber is

$$
r_{e}^{*}=\sqrt{\frac{\left[\omega_{x}\right]_{V}}{\left[\omega_{x}\right]_{H}}}=\sqrt{\frac{\left[T_{x}\right]_{V}}{\left[T_{x}\right]_{H}}}
$$

where $\left[T_{x}\right]_{V}$ and $\left[T_{x}\right]_{H}$ represent the torques when the fiber is fixed in the vertical and horizontal directions, respectively. Cox [41] used the slender body theory to calculate the torques in two directions and proposed a closed-form formula, but this formula is only applicable to long cylindrical fibers, not short cylindrical fibers.

In this chapter, we use the Finite Element Method to calculate the hydrodynamic torques exerted on the fiber in the vertical and horizontal orientations, respectively, with the velocity boundary condition in Eq.(2.5) as $\mathbf{U}_{\mathrm{BC} 3}=\mathbf{0}$. In this case, a fiber is fixed within the fluid without translation and rotation, and $r_{e}^{*}$ is computed with Eq.(2.17).

\section{$2.4 \quad$ Implementation and examples}

We implement our numerical schemes using COMSOL (COMSOL, Inc., Burlington, MA, USA) and Matlab (The MathWorks, Inc., Natick, MA, USA). COMSOL solves Eqs.(2.1)-(2.5), and Matlab is used to search fiber velocities and update fiber position and orientation at each time $t_{i}$ in the Runge-Kutta solution. In this section, several examples are presented to illustrate and validate the proposed approach. 


\subsubsection{Validation of Jeffery's orbit}

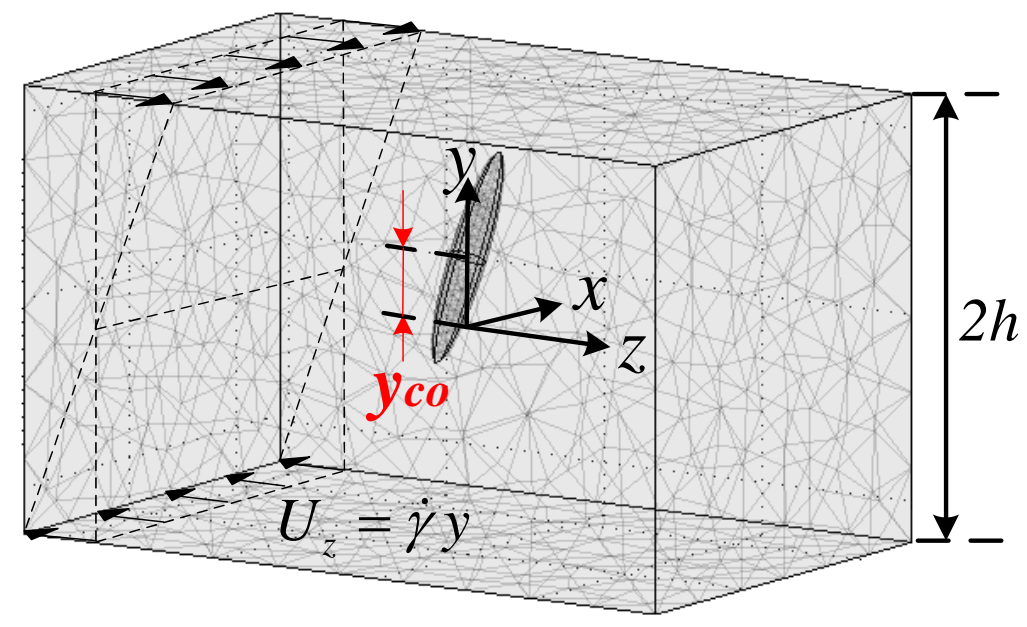

Figure 2.3: Finite element model of a single ellipsoidal fiber in a simple shear flow

We use the proposed computational methodology to evaluate the motion of a single ellipsoidal fiber in a simple shear flow, and compare with Jeffery's theoretical solution. The problem is set up in Fig.2.3, with the parameters tabulated in Table 2.1 , where $a$ and $b$ are the long and short axes of an ellipsoidal fiber, respectively, and $h$ is the half distance between the upper and lower boundary of our computational domain, which is much larger than the fiber size.

\begin{tabular}{|l|l|}
\hline Parameters & Value \\
\hline Geometric aspect ratio of the ellipsoid & $r_{e}=\frac{a}{b}=6$ \\
\hline Undisturbed simple shear flow & $U_{z}=\dot{\gamma} y$, where $\dot{\gamma}=1 \mathrm{~s}^{-1}$ \\
\hline Raito of fiber size to fluid domain dize & $\varepsilon=2 h / a=40 \gg 1$ \\
\hline Initial fiber orientation/position & $\begin{array}{l}\theta_{0}=\pi / 3(\mathrm{rad}), \phi_{0}=\psi_{0}=0(\mathrm{rad}) \\
y_{c 0}=0.1(\mathrm{~mm}), x_{c 0}=z_{c 0}=0(\mathrm{~mm})\end{array}$ \\
\hline
\end{tabular}

Table 2.1: Parameters to simulate an ellipsoidal fiber motion

The motion of a single ellipsoidal fiber using the proposed methodology is compared with Jeffery's solution in Figs.2.4 and 2.5. In this example, we apply $\Delta t=0.2 \mathrm{~s}$ in the Runge-Kutta method. The initial $y_{c 0}$ equals $0.1 \mathrm{~mm}$, so the initial linear velocity of fiber centroid in the $z$ direction is $\dot{z}_{c 0}=\dot{\gamma} y_{c 0}=0.1 \mathrm{~mm} / \mathrm{s}$. Figure 2.4 shows 

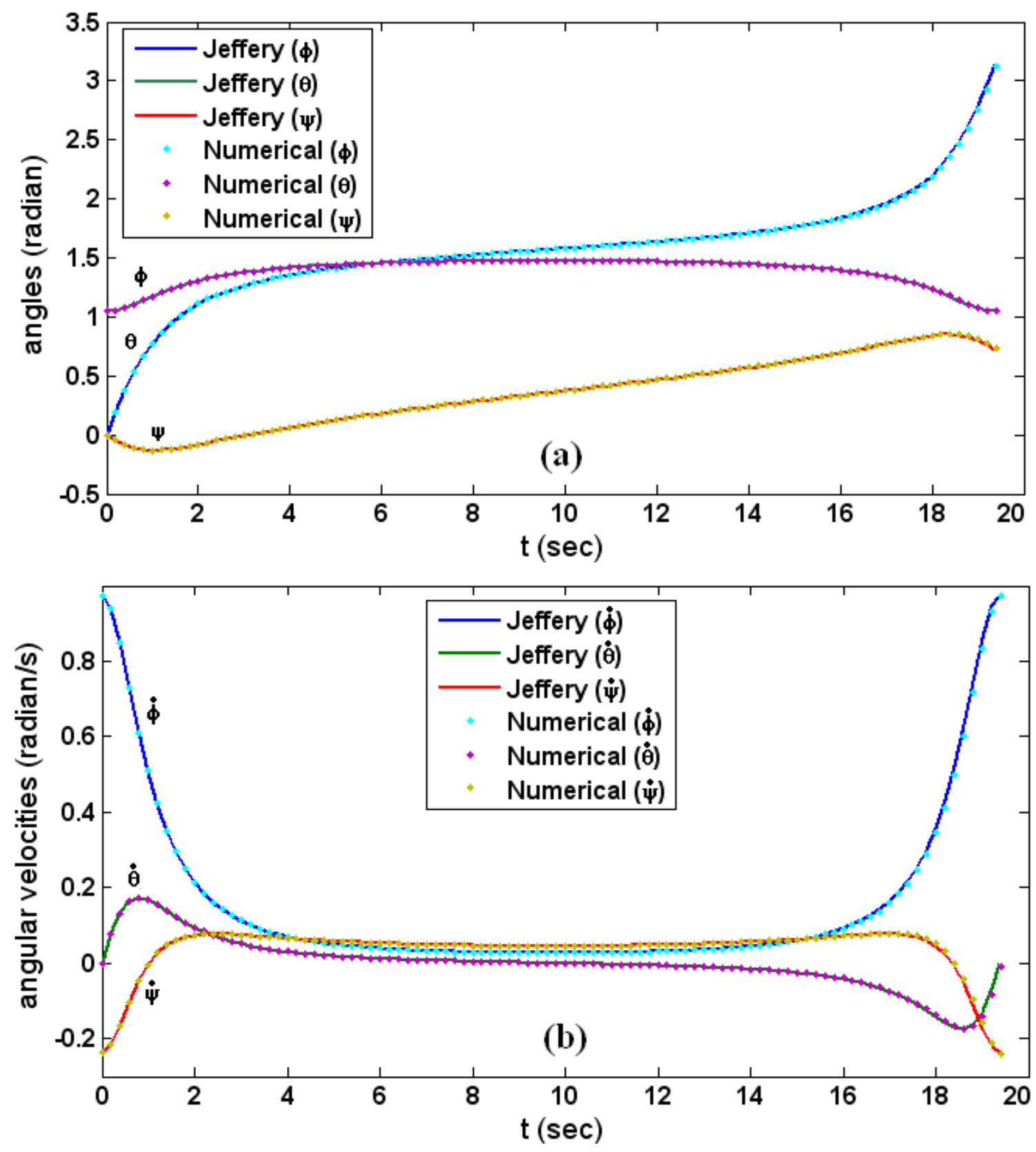

Figure 2.4: Three-dimensional single fiber orientation in half period: (a) Evolution of $\phi, \theta, \psi$ and (b) Evolution of $\dot{\phi}, \dot{\theta}, \dot{\psi}$

that the fiber orientation agrees with Jeffery's equation and Fig.2.5 illustrates that the fiber centroid translates with the same linear velocity as the undisturbed fluid evaluated at the fiber centroid. The comparison of two sets of data, i.e., Jeffery's data and our numerical data appear in Table 2.2, where the average relative error $E_{R}$ and 

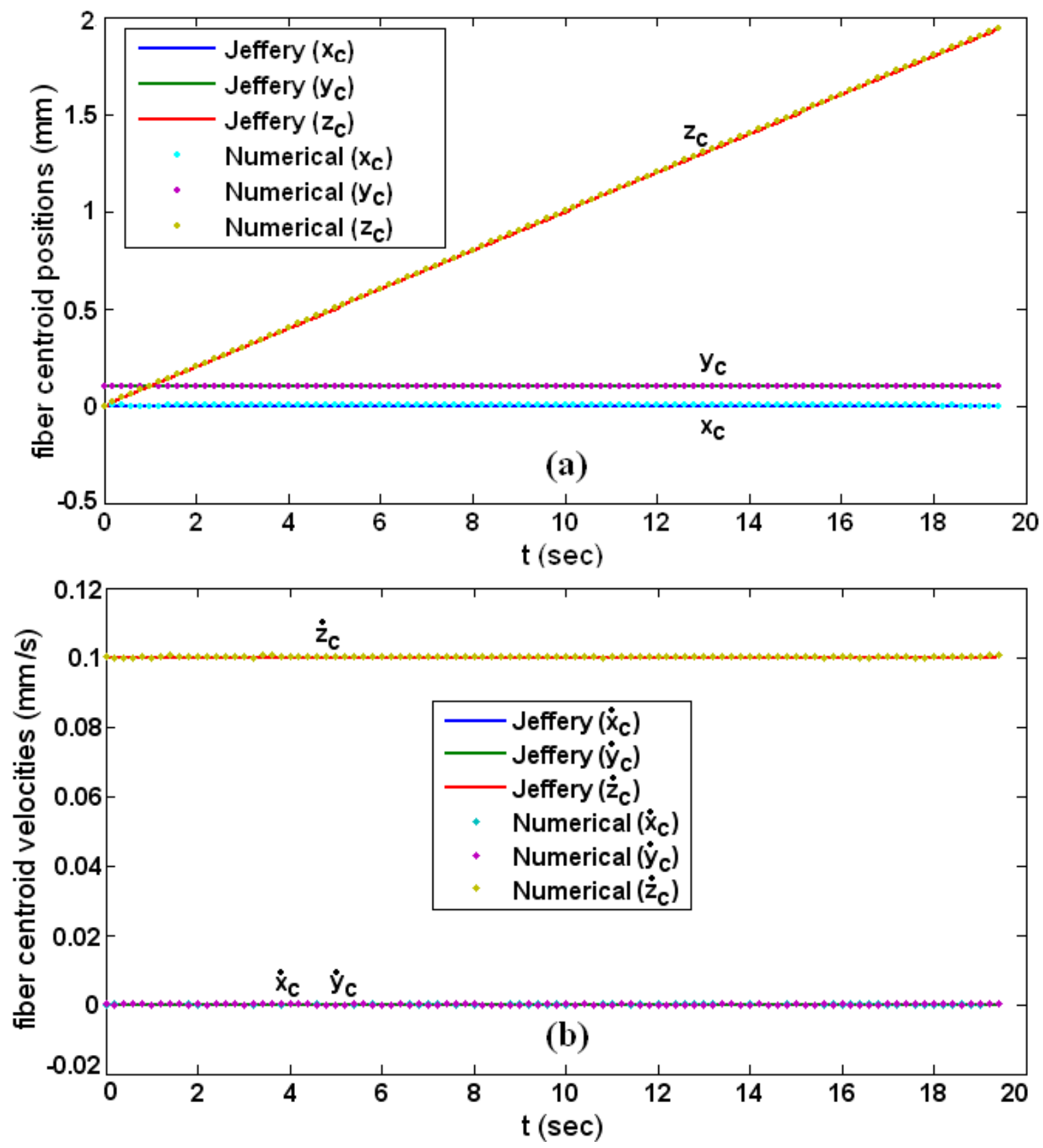

Figure 2.5: Three-dimensional single fiber translation in half period: (a) Evolution of $x_{c}, y_{c}, z_{c}$ and (b) Evolution of $\dot{x}_{c}, \dot{y}_{c}, \dot{z}_{c}$

absolute error $E_{A}$ are, respectively, computed over $N$ time steps as

$$
\begin{aligned}
& E_{R}=\left[\frac{1}{N} \sum_{i=1}^{N}\left|A_{i}-B_{i}\right|\right] /\left[\frac{1}{N} \sum_{i=1}^{N}\left|A_{i}\right|\right] \\
& E_{A}=\frac{1}{N} \Sigma_{i=1}^{N}\left|A_{i}-B_{i}\right|
\end{aligned}
$$


where $A_{i}$ and $B_{i}$ are, respectively, Jeffery's data and our numerical data in each time step $t_{i}$. The relative error is not available for $x_{c}$, because the average of true data (Jeffery's solution) for $x_{c}$ is zero.

\begin{tabular}{|c|c|c|}
\hline Data Sets & $\mathbf{E}_{\mathbf{R}}$ & $\mathbf{E}_{\mathbf{A}}$ \\
\hline \hline$\phi($ radian $)$ & $4.6 \mathrm{E}-5$ & $7.2 \mathrm{E}-5$ \\
\hline$\theta($ radian $)$ & $1.1 \mathrm{E}-5$ & $1.6 \mathrm{E}-5$ \\
\hline$\psi($ radian $)$ & $9.8 \mathrm{E}-5$ & $3.5 \mathrm{E}-5$ \\
\hline$x_{c}(\mathrm{~mm})$ & $-N A-$ & $6.0 \mathrm{E}-5$ \\
\hline$y_{c}(\mathrm{~mm})$ & 0.0017 & $17 \mathrm{E}-5$ \\
\hline$z_{c}(\mathrm{~mm})$ & 0.0016 & 0.0016 \\
\hline
\end{tabular}

Table 2.2: Comparison of the results between Jeffery's theory and our approach

In order to verify Jeffery's orbit, we maintain the following conditions within our finite element model, which are analogous to Jeffery's assumptions:

a) The flow domain is much larger than the fiber size. Jeffery assumed the unbounded fluid domain in his paper [8], so we set $\varepsilon=2 h / a=40$, which provides a good agreement with Jeffery's results. Our simulations show that when $\varepsilon>20$, the results are stable. More discussions on fiber motions in a bounded fluid domain can be seen in Chapter 5.

b) The local particle Reynolds number is computed as $\rho U(a) / \mu \ll 1$, where $U$ represents the characteristic velocity. In the proposed simulation, the parameters are set up to guarantee the creeping flow condition, which models the polymer composites processing. So the convection term $\rho \mathbf{U} \cdot \nabla \mathbf{U}$ does not have any effect on the results. The excellent agreement between Jeffery's theory and our approach validates the accuracy and robustness of our approach. More importantly, our approach is a systematic methodology which is extended to study different shapes of fiber and other complex flow conditions, which were not addressed in Jeffery's original work. 


\subsubsection{Equivalent aspect ratio - general axisymmetric fiber}

In this section, we consider the motions of other axisymmetric fibers, and numerically determine the equivalent aspect ratios of axisymmetric fibers, including ellipsoidal, cylindrical, and bead-chain fibers over a large range of aspect ratios.

\section{Ellipsoidal fibers}

The concept of equivalent aspect ratio is proposed based on the analysis of an ellipsoidal fiber, as seen in Eq.(2.17). Therefore, the equivalent aspect ratio of an ellipsoidal fiber is expected to be the same as its geometric aspect ratio, which is validated by our numerical data tabulated in Table 2.3 .

\begin{tabular}{|c|c|c|}
\hline $\begin{array}{c}\text { Geometric Aspect Ratio } \\
\left(r_{e}\right)\end{array}$ & $\begin{array}{c}\text { Equivalent Aspect Ratio } \\
\left(r_{e}^{*}\right)\end{array}$ & $\begin{array}{c}\text { Relative } \\
\text { Error }\end{array}$ \\
\hline \hline 1 & 1.01 & 0.01 \\
\hline 5 & 5.01 & 0.0016 \\
\hline 10 & 9.91 & 0.009 \\
\hline 15 & 14.85 & 0.01 \\
\hline 20 & 20.15 & 0.007 \\
\hline 30 & 30 & $3.9 \mathrm{E}-5$ \\
\hline 40 & 39.60 & 0.01 \\
\hline 50 & 49.90 & 0.002 \\
\hline
\end{tabular}

Table 2.3: Equivalent aspect ratios of ellipsoidal fibers

\section{Cylindrical fibers}

For a cylindrical fiber, the geometric aspect ratio is defined as the ratio of fiber length to its diameter. The equivalent aspect ratio of a cylinder is calculated with Eq.(2.17), and the torques in two directions, shown in Fig.2.6, are evaluated using the proposed numerical method.

The relationship between the equivalent aspect ratio $r_{e}^{*}$ and geometric aspect ratio $r_{e}$ is shown in Fig.2.7. For cylindrical fibers, we plot our numerical data with that 


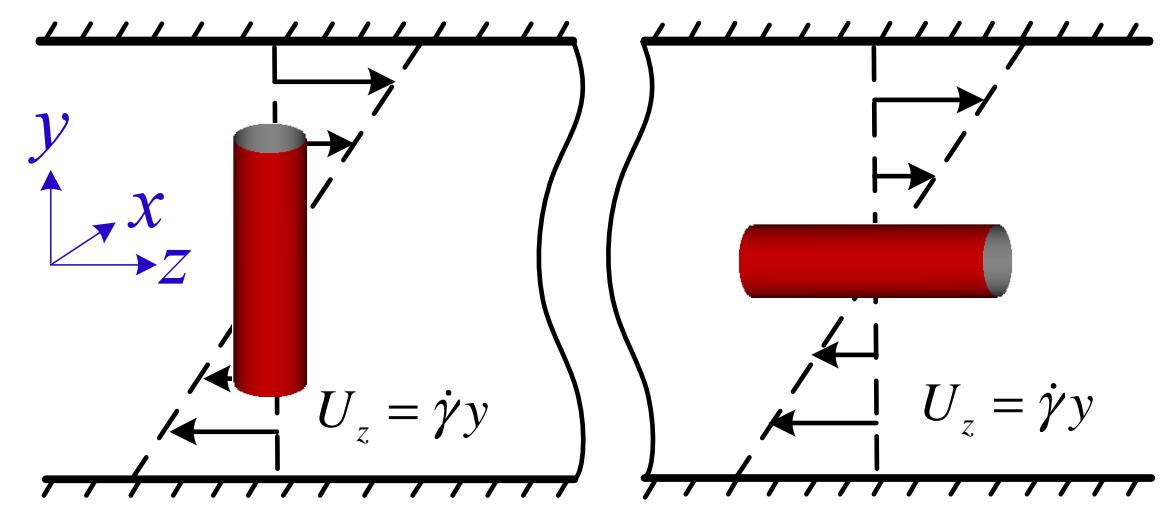

Figure 2.6: Torques on a cylindrical fiber fixed in two directions: (a) The vertical direction and $(b)$ the horizontal direction

from Cox's equation [41] and experiments [31-33, 39, 43]. Cox [41] used the slender body theory to calculate the torques so as to obtain the equivalent aspect ratio of a cylinder from Eq.(2.17), with the expression as

$$
r_{e}^{*}=\frac{1.24 r_{e}}{\sqrt{\ln \left(r_{e}\right)}}
$$

However, Eq.(2.20) is only applicable to long rod-like fibers, not short cylindrical fibers due to the use of the slender body theory. Therefore, this formula is ineffective for fibers used in short-fiber reinforced composite materials.

From Fig.2.7, we can see that for long fibers, our data matches well with Cox's theory [41] and conforms to the experimental data [31-33, 39, 43]. We use a cubic polynomial to fit our numerical data in Fig.2.7 to obtain the closed-form expression

$$
r_{e}^{*}=0.000035 r_{e}^{3}-0.00467 r_{e}^{2}+0.764 r_{e}+0.404
$$

with the fitting correlation coefficient 0.99998. Equation (2.21) is applicable to both short and long cylindrical fibers with the geometric aspect ratios from 1 to 50 . 


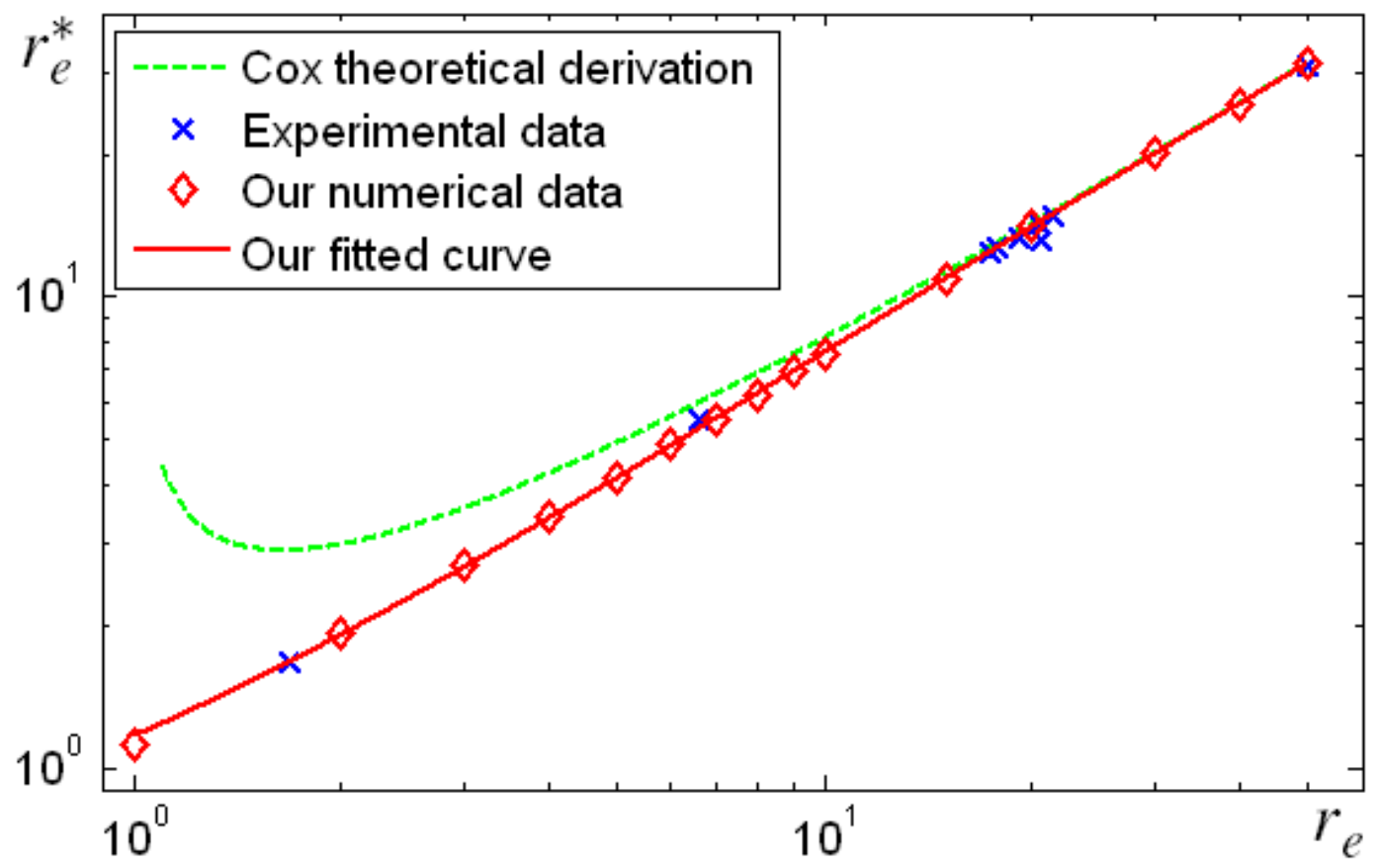

Figure 2.7: Equivalent aspect ratios of cylinders: the comparison of our numerical data (red diamonds) and fitted curve (red line) with Cox's theoretical curve (dashed green curve) for slender fibers and experimental data (blue crosses)

\section{Bead-chain fibers}

Bead-chain fibers are commonly used to model flexible fibers. Hence, the study of equivalent aspect ratios of bead-chain fibers provides insight into the study of the motion of flexible fibers. For a bead-chain fiber, the geometric aspect ratio is defined as the number of beads connected together. For instance, the geometric aspect ratio is four in Fig.2.8. We numerically obtained the torques when the fiber is fixed as shown in Fig.2.8 using the proposed computational approach.

Equation (2.17) is used to obtain the numerical data of equivalent aspect ratios of bead-chain fibers, as shown in Fig.2.9, from which we see that the equivalent aspect ratio of a bead-chain fiber is different from its geometric aspect ratio. As the fiber gets longer, the difference becomes larger. By fitting our numerical data in Fig.2.9, we propose the following fitted formula to calculate the equivalent aspect ratio, shown 


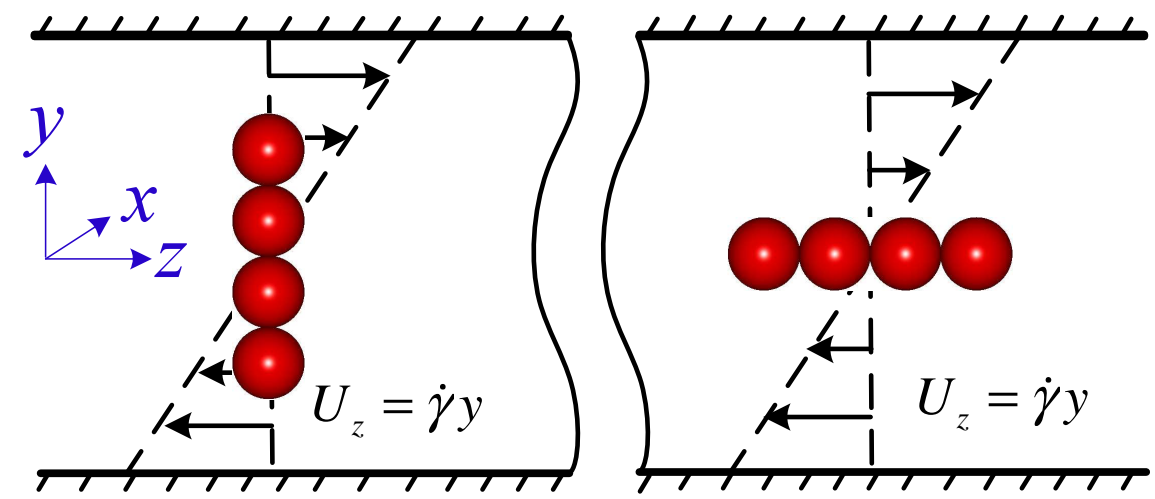

Figure 2.8: Torques on a bead-chain fiber fixed in two directions: $(a)$ The vertical direction and $(b)$ the horizontal direction

as

$$
r_{e}^{*}=-0.005 r_{e}^{2}+0.902 r_{e}+0.145
$$

with the fitting correlation coefficient 0.99965. Note that for our fitted data, the geometric aspect ratios are from 1 to 30.

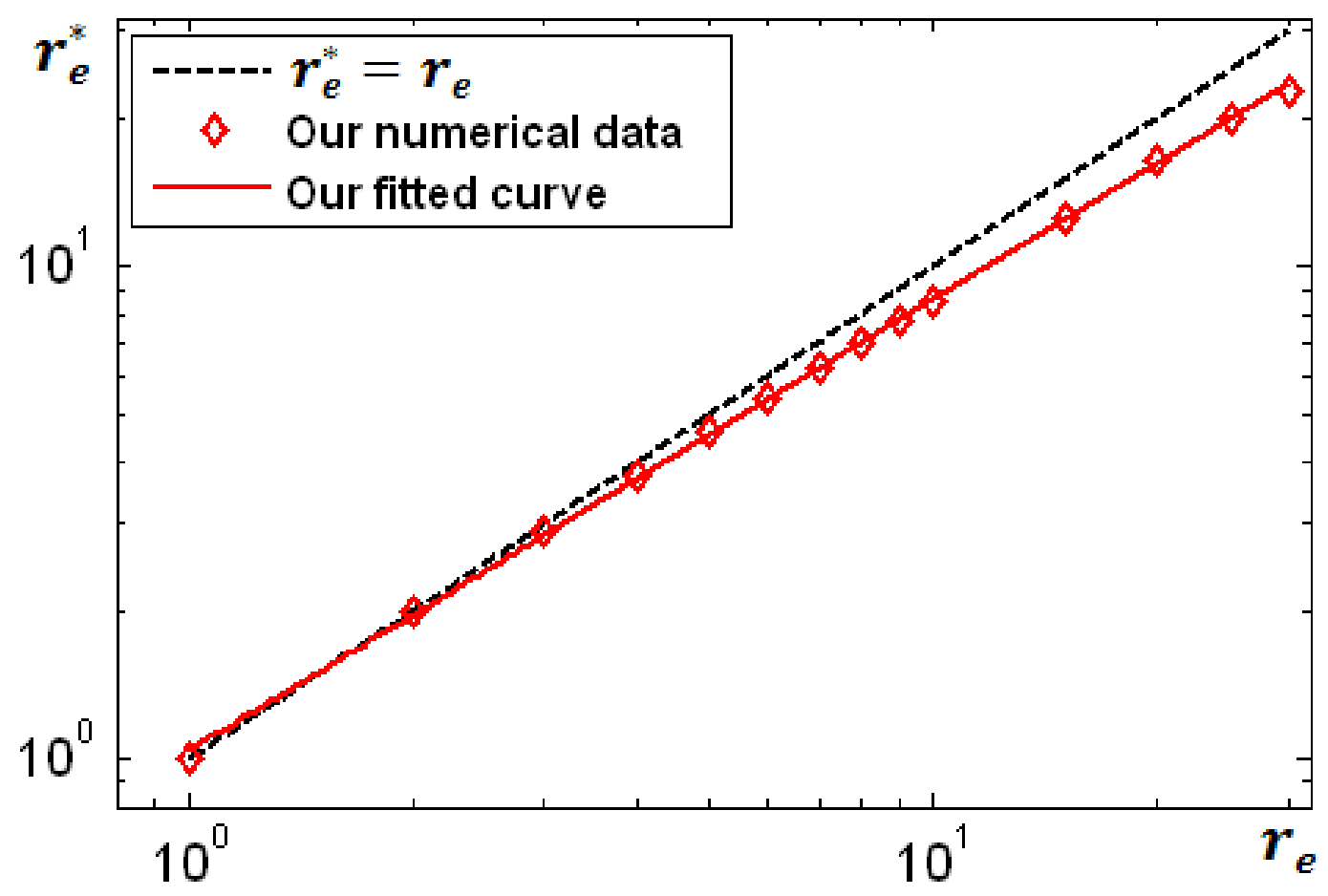

Figure 2.9: Equivalent aspect ratios of bead-chain fibers: red diamonds represent our numerical data and red line is our fitted curve of the data

We notice that in current research on flexible fibers using the bead-chain model 


\begin{tabular}{|c|c|c|}
\hline $\begin{array}{c}\text { Geometric Aspect Ratio } \\
\left(r_{e}\right)\end{array}$ & $\begin{array}{c}\text { Including Beads } \\
\left(r_{e}^{*}\right)\end{array}$ & $\begin{array}{c}\text { Isolated Beads } \\
\left(r_{e}^{*}\right)\end{array}$ \\
\hline 2 & 2.0 & 1.6 \\
\hline \hline 4 & 3.7 & 3.0 \\
\hline 6 & 5.4 & 4.4 \\
\hline 8 & 7.0 & 5.8 \\
\hline 10 & 8.6 & 7.2 \\
\hline
\end{tabular}

Table 2.4: Comparison of two bead-chain models

[26], the force and torque on the fiber are calculated based on each separate sphere independently, i.e., when calculating the force and torque on the top sphere, it is assumed that there are no other connecting spheres around the top sphere within the fluid. This assumption is not valid in reality, due to the flow shielding from the neighboring spheres causing the change in the local velocity profile of the fluid, which in turn changes the force and torque distribution on the exterior surface of the top sphere. Accounting for the flow shielding should better represent the physical system that bead-chain models approximate. The results from our study for the equivalent aspect ratios of bead-chain fibers are provided in Table 2.4 for both scenarios with and without flow shielding. It can be seen that assuming isolated beads does not reflect the real equivalent aspect ratios of bead-chain fibers.

\section{Comparison of equivalent aspect ratios}

We summarize the equivalent aspect ratios of various axisymmetric fibers, all having a geometric aspect ratio $r_{e}=6$, as shown in Table 2.5.

Note that even though the geometric aspect ratios of different fibers are the same, the equivalent aspect ratios are quite distinct, resulting in the different fiber motions. 


\begin{tabular}{|c|c|c|c|c|c|}
\hline $\begin{array}{c}\text { Fiber } \\
\text { Shapes }\end{array}$ & Ellipsoid & Cylinder & Bead-chain & $\begin{array}{l}\text { Cylinder with } \\
\text { round ends }\end{array}$ & $\begin{array}{l}\text { Two half } \\
\text { ellipsoids }\end{array}$ \\
\hline FEM & $b \geqslant a$ & 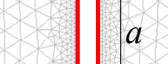 & $8 a$ & $a$ & \\
\hline$r_{e}(a / b)$ & $r_{e}=6$ & $r_{e}=6$ & $r_{e}=6$ & $r_{e}=6$ & $r_{e}=6$ \\
\hline$r_{e}^{*}$ & $r_{e}^{*}=6$ & $r_{e}^{*}=4.8$ & $r_{e}^{*}=5.4$ & $r_{e}^{*}=5.1$ & $r_{e}^{*}=5.2$ \\
\hline
\end{tabular}

Table 2.5: Comparison of equivalent aspect ratios of fibers with various shapes

\subsubsection{Validation of equivalent aspect ratio}

In this section, we use the methodology, proposed in Sec.2.2 to solve the motions of a cylindrical fiber and a bead-chain fiber, so as to validate our numerical data for equivalent aspect ratios.

\section{Cylindrical fibers}

In this example, we consider $r_{e}=6$ for a cylindrical fiber. The shear rate of the undisturbed simple shear flow is $1 \mathrm{~s}^{-1}$, and the initial conditions are $\theta_{0}=\pi / 3(\mathrm{rad})$, $\phi_{0}=\psi_{0}=0(\mathrm{rad})$ and $x_{0}=y_{0}=z_{0}=0(\mathrm{~mm})$. The evolution of $\phi, \theta, \psi$ and $\dot{\phi}, \dot{\theta}, \dot{\psi}$ are shown in Fig.2.10, in which the orientation of a cylindrical fiber is obtained using the methodology proposed in Sec.2.2, while the orientation of an ellipsoidal fiber is evaluated from Jeffery's analytical formula.

Figure 2.10 shows that a cylinder with $r_{e}=6$ has the same rotation as that of an ellipsoid with $r_{e}=4.8$, i.e., the equivalent aspect ratio of a cylinder with $r_{e}=6$ is 4.8. Therefore, the equivalent aspect ratios obtained using our computations with Eq.(2.17) are validated. 

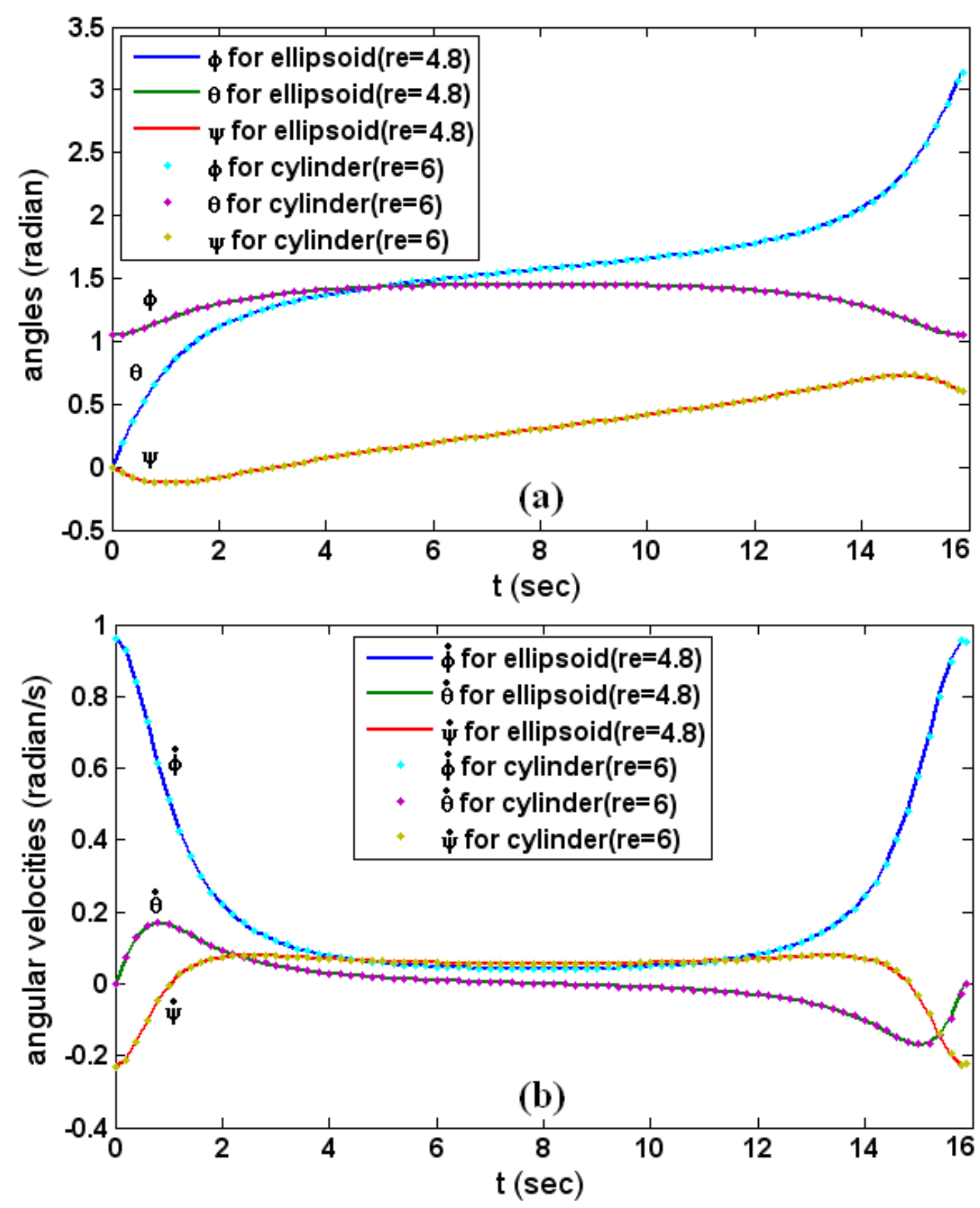

Figure 2.10: Evolution of a cylindrical fiber with geometric aspect ratio $r_{e}=6$ and an ellipsoidal fiber with $r_{e}=4.8$ : (a) Evolution of $\phi, \theta, \psi ;(b)$ Evolution of $\dot{\phi}, \dot{\theta}, \dot{\psi}$

\section{Bead-chain fibers}

Similarly, we evaluate the motion of a bead-chain fiber with $r_{e}=6$. The shear rate of the undisturbed simple shear flow is $1 s^{-1}$, and the initial conditions are $\theta_{0}=$ 

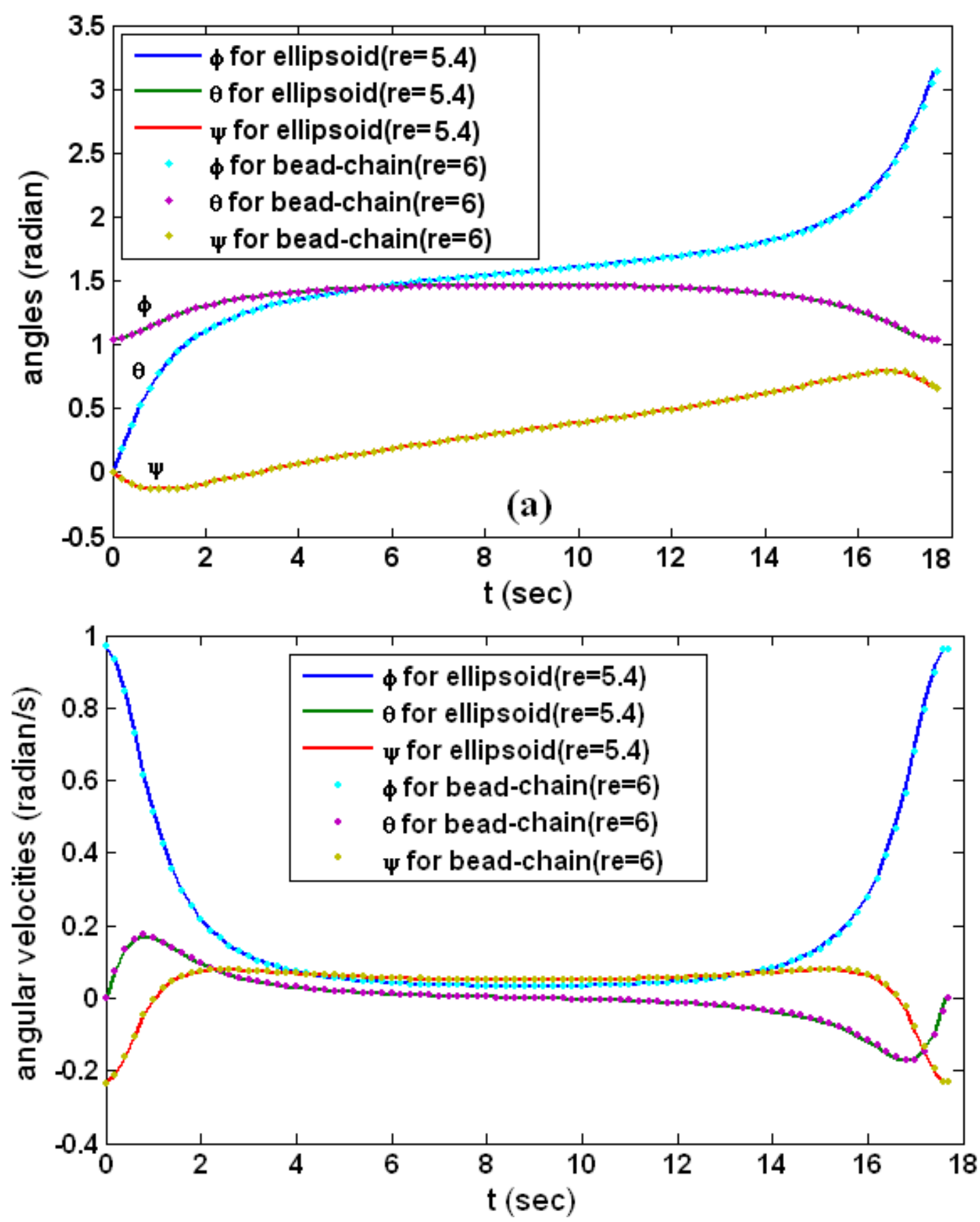

Figure 2.11: Evolution of a bead-chain fiber with the geometric aspect ratio $r_{e}=6$ and an ellipsoidal fiber with $r_{e}=5.4$ : (a) Evolution of $\phi, \theta, \psi ;(b)$ Evolution of $\dot{\phi}, \dot{\theta}, \dot{\psi}$

$\pi / 3(\mathrm{rad}), \phi_{0}=\psi_{0}=0(\mathrm{rad})$ and $x_{0}=y_{0}=z_{0}=0(\mathrm{~mm})$. The results are shown in Fig.2.11, in which the motion of a bead-chain fiber is evaluated by our numerical schemes while the motion of an ellipsoidal fiber is obtained from Jeffery's analytical 
solution. From Fig.2.11, we can see the bead-chain fiber with the geometric aspect ratio $r_{e}=6$ has the same rotational movement as that of the ellipsoidal fiber with $r_{e}=5.4$, which validates our numerical data for equivalent aspect ratios of bead-chain fibers.

\section{Comparison of the motions of fibers with different shapes}

With the same geometric aspect ratio $\left(r_{e}=6\right)$, ellipsoidal, cylindrical and bead-chain fibers have different motions, compared in Fig.2.12.

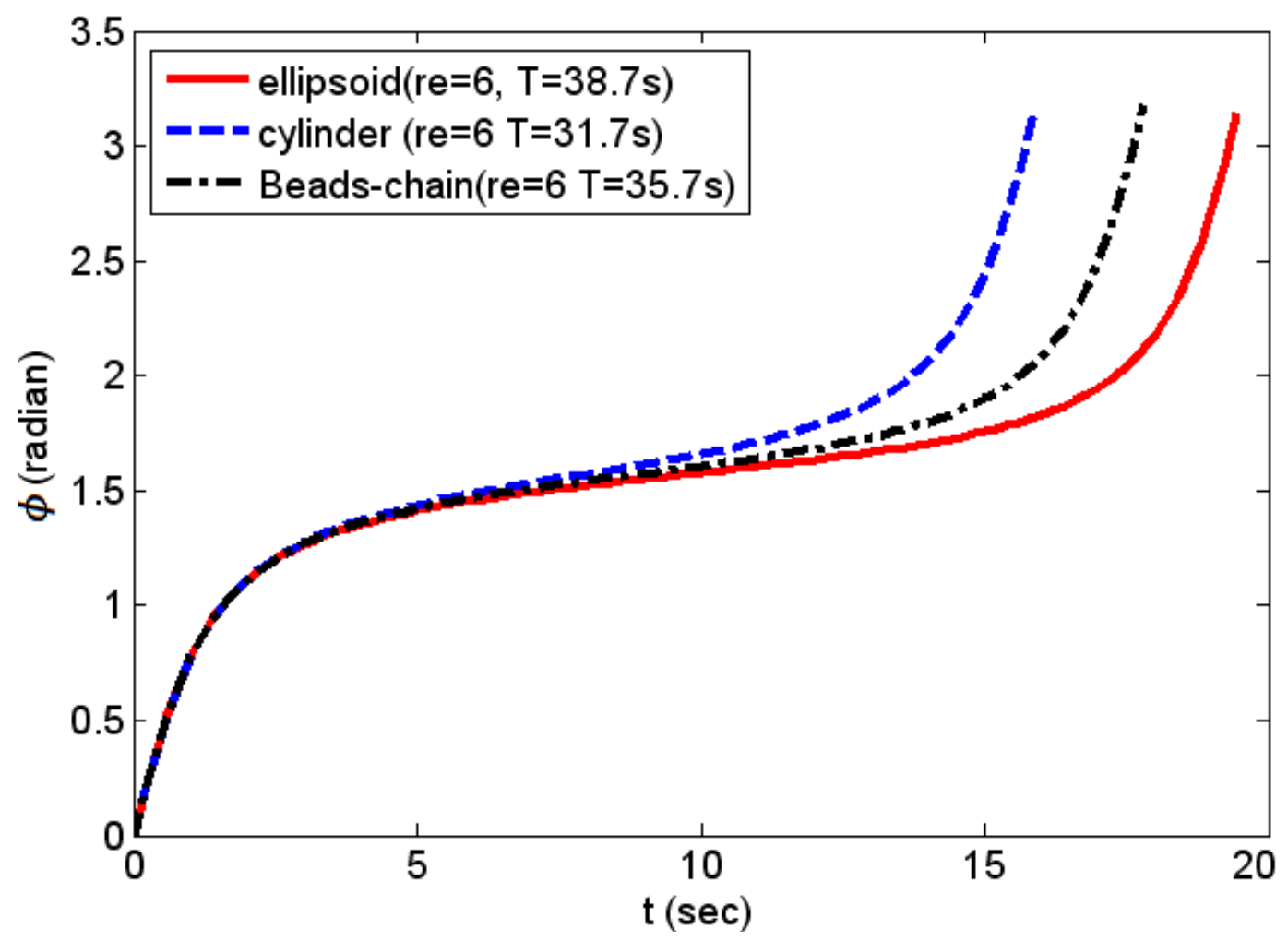

Figure 2.12: Comparison of the motions of ellipsoidal, cylindrical and bead-chain fibers with the same geometric aspect ratio $r_{e}=6$ (period $\mathrm{T}$ is given as shown)

We can see that an ellipsoid has the longest period compared with the cylindrical and bead-chain fibers. The cylindrical fiber has the shortest period among these three shapes, such that we would expect the cylinder to align quickly with the flow than the other shapes. Our results indicate that the impact of fiber shape on the fiber 
motion is significant, but fiber shape alone likely does not support the slow motions observed in $[30,34]$. Other effects such as wall effect and fiber-fiber interactions could be the reasons for the earlier observations, which are further discussed in Chapter 5 .

\subsection{Summary}

A methodology, which combines the Finite Element Method, a Newton-Raphson iteration and a Runge-Kutta method, is presented to solve the general three-dimensional motion of a single suspended fiber within a viscous fluid. This method is very general and can be applied to any axisymmetric fiber, and in the present study results are presented for ellipsoidal, cylindrical and bead-chain fibers. We demonstrate that fiber shape has a significant impact on the fiber orientation, which affects the rate of fiber alignment in short-fiber reinforced composite materials. For an axisymmetric fiber, the equivalent aspect ratio is needed for numerical solutions of the Jeffery's equation for the fiber motion instead of the geometric aspect ratio. The equivalent aspect ratios of cylindrical fibers and other fiber types are generated using our Finite Element Method, and are validated by evaluating the fiber motion numerically.

In this research work, the commercial FEM solver COMSOL is used and the Navier-Stokes equation is solved to obtain the velocity and pressure in the bulk fluid, and then the force and torque on fiber. But there are three factors limiting the time efficiency of the simulation: 1) Finite element model is invisible to the users, so the Jacobian matrix in the Newton-Raphson method can only be obtained using the finite difference method, which causes huge computation time; 2) The convection term of Navier-Stokes equation in COMSOL cannot be switched off, which causes more computation time; 3) With different fiber orientations, the FEM solver has to be called to solve fluid velocity and pressure. so the same type of system of equations has to be solved repeatedly, which accumulates the computation time. Therefore, the 
simulation using commercial FEM solver cannot meet the demand for the study of fiber motion in complex flow fields, such as Poiseuille flows. 


\section{Chapter 3}

\section{Stand-Alone FEM Package Using Analytical Jacobian and Transformed Essential Boundary}

\subsection{Introduction}

In Chapter 2, we investigate a single fiber motion within a viscous flow using COMSOL Multiphysics. But considering the study of fiber motion in inhomogeneous flows, Brownian motion of nano-fibers and fiber-fiber interactions, the simulation using COMSOL Multiphysics might not be efficient, because the finite element model is invisible to users. Therefore, we proposed a stand-alone Finite Element Method to study the fiber motion in various flows, including homogenous flows (simple shear flow, rotational flow and extensional flow) and Poiseuille flows. Flow of polymer melt within a mold cavity is treated as a pressure-drive flow or Poiseuille flow. The study of fiber migration in a Poiseuille flow dates back to the $19^{\text {th }}$ century, where Segré and Silberberg [44] experimentally observed that spherical particles would migrate to an equilibrium annular region about 0.6 tube radii from the tube axis. This finding has

spawned a great number of experimental [45-49] and theoretical [50-53] studies of 
fiber suspensions in a Poiseuille flow.

On the experimental side, Karnis $[46,47]$ performed the study on rods and discs and suggested that lateral migration could result from the deformation of particles. Oliver [48] conducted the similar experiments of fiber suspensions, with the tube Reynolds number in the range of 100-500, and the relative particle Reynolds number in the range of 1.0-13.0. He also observed the lateral migration of particles. Repetti [49] first studied the lateral migration in a plane Poiseuille flow, and gave a possible explanation of Segré-Silberberg phenomenon by means of Rubinow and Keller's theory [50]. Tachibana [54] reported from his experiments that the equilibrium positions for two-dimensional and three-dimensional Poiseuille flows are identical.

On the theoretical side, Rubinow [50] and Saffman [51] studied the lift force in viscous Poiseuille flows, but they did not take account of the boundary wall. Cox and Brenner [52] used a matched asymptotic expansion to analyze lateral particle movement in a three-dimensional Poiseuille flow in a tube. Vasseur [55] extended the method proposed by Cox and Brenner [52] to the case in which the flow is bounded by two vertical parallel plates. Ho and Leal [53] also analyzed the lateral migration using the method of reflexions, which is similar to Cox and Brenner's method [52]. Schonberg [56] extended Saffman's work [51] and analyzed the change of equilibrium position of fibers in Poiseuille flows as the tube Reynolds number increases.

For the creeping flow, where the inertial term is ignored, no lateral force could arise. This has been demonstrated theoretically by Bretherton [38] and Chwang [57], and experimentally by Goldsmith [45]. Bretherton [38] discussed the SegréSilberberg phenomenon and concluded that this phenomenon must result from some kinds of non-linear effects, such as inertial or non-Newtonian forces. Chwang [57] used the singularity method to develop a close-form solution for the fiber motion in quadratic Stokes flows. He proposed that fiber orientation would follow Jeffery's orbital equation with the shear rate evaluated at fiber centroid. He also suggested that 
the fiber translates along a straight path parallel to the main flow having a variable translation speed. Goldsmith [45] conducted the experiments in circular Poiseuille flows at much low Reynolds numbers $\left(<10^{-6}\right)$, and found no lateral migration of fibers. Karnis and Goldsmith [47] also observed in experiments that fibers with different shapes, such as rods and discs, would follow Jeffery's orbit and lag behind the undisturbed fluid velocities at all radial positions, which agrees with Chwang's theory. Stover [58] also experimentally showed that fiber's tumbling motion is in excellent agreement with Jeffery's orbit, but the slow linear motion was not observed in his experiments.

With the development of Computational Fluid Dynamics (CFD), the numerical simulation of fiber migration in a viscous fluid provides additional insight into fiber motion [59-63]. Sugihara-Seki [59, 60] solved the fiber motion in Stokes flows without considering fiber inertia by using the Finite Element Method. The fiber rotation is expected to be the same as Jeffery's orbit $[45,57]$, however his data deviates from Jeffery's orbit. We note that Sugihara-Seki's work [59] was for the planar fiber motion i.e. fiber only moves in one plane, which is not a real three-dimensional migration. Feng [61] used Hu's method [62] to simulate the fiber motion using a two-dimensional finite element simulation. Feng [61] analyzed the non-linear inertial effects in his simulation, and observed the Segré-Silberberg phenomenon in Poiseuille flows. Clague [63] used the lattice Boltzmann (LB) method to calculate the force and torque on a sphere in a Poiseuille flow.

We propose a three-dimensional Finite Element Method to solve the fiber motion in three-dimensional space. By making force and torque exerted on fiber to be zero, we use a Newton-Raphson method to calculate fiber's linear and angular velocities. We develop an analytical Jacobian matrix used in the Newton-Raphson method. Then a $4^{\text {th }}$-order Runge-Kutta method is utilized to track the position and orientation of fiber as functions of time. A method to transform essential boundary condition on 
the fluid boundary is proposed, which avoids the re-meshing of the fluid domain at each time instant. Hence, only one mesh model and stiffness matrix are needed. To our best knowledge, we are the first to solve the full fiber motion in three-dimensional space using a three-dimensional Finite Element Method.

The remainder of this chapter is organized as follows: Section 3.2 gives the background related to the prediction of fiber motion in Poiseuille flows. The proposed stand-alone FEM-based simulation tool is elucidated in Sec.3.3, followed by the implementation and examples in Sec.3.4. Summary is given in Sec.3.5.

\subsection{Chwang's model for Poiseuille flows}

Poiseuille flows are one of inhomogeneous flows, i.e. velocity profile is related to the square of the spatial dimension, and the flow field is driven by the constant pressure difference in certain direction. Poiseuille flows are categorized into two types: a plane Poiseuille flow between two parallel plates and a circular Poiseuille flow in a tube, as seen in Fig.3.1.

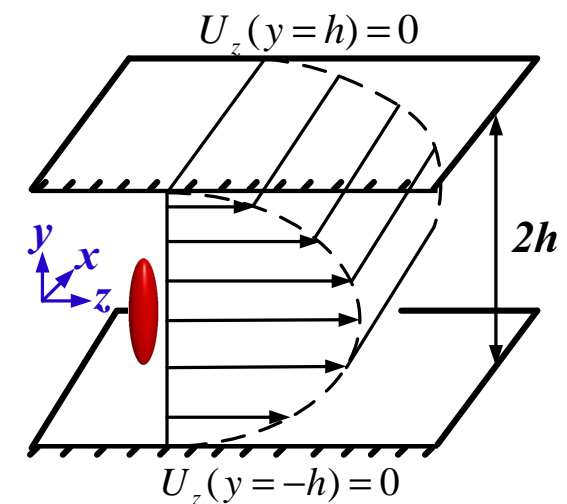

(a)

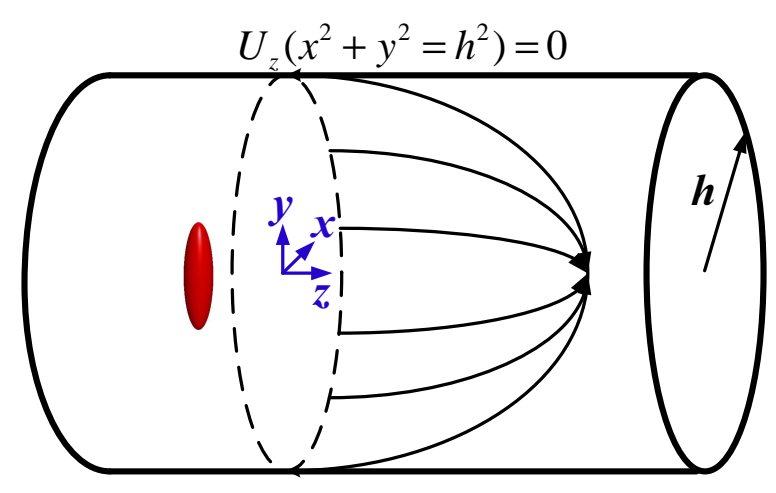

(b)

Figure 3.1: Two types of Poiseuille flows: (a) Plane Poiseuille flow between two parallel plates and $(b)$ circular Poiseuille flow in a tube 
The plane Poiseuille flow in Fig.3.1(a) is defined as:

$$
U_{x}=U_{y}=0, U_{z}=\frac{\nabla p}{2 \mu}\left[h^{2}-y^{2}\right]
$$

and the circular Poiseuille flow in Fig.3.1(b) is defined as:

$$
U_{x}=U_{y}=0, U_{z}=\frac{\nabla p}{4 \mu}\left[h^{2}-x^{2}-y^{2}\right]
$$

where $\nabla p$ is the constant pressure gradient in the $z$ direction and $h$ represents the half distance between two parallel plates in Fig.3.1( $a$ ) and radius of the circular tube in Fig.3.1(b).

Chwang [57] used the singularity method to study fiber motion in various unbounded incompressible quadratic flows. He decomposed the general quadratic flow into the local coordinate system with the origin located at fiber centroid. For a plane Poiseuille flow, the flow field is originally defined in the $x y z$ coordinate system (cf.Eq.(3.1)), and then transformed into the $\xi \eta \zeta$ system with the origin located at fiber centroid, as illustrated in Fig.3.2, in which $y_{c}$ is the $y$ coordinate of fiber centroid in the $x y z$ system.

For the case of a simple shear flow, the velocity field can be decomposed into a uniform flow and a simple shear flow with the fiber centroid located at the origin of the $x y z$ system (as seen in Fig.3.2 top). The uniform flow component moves the fiber along with the fluid, and the simple shear flow component causes the fiber to rotate. In a similar manner, a Poiseuille flow is composed of a uniform flow, a simple shear flow and a quadratic flow (as seen in Fig.3.2 bottom). Only the shear flow component contributes to the fiber rotation, while the uniform and quadric flow components push the fiber forward or drag the fiber backward. Chwang [57] derived the resultant force and torque exerted on the fiber as the sum of the forced from those three flow components, and then calculated the fiber velocities, which make 


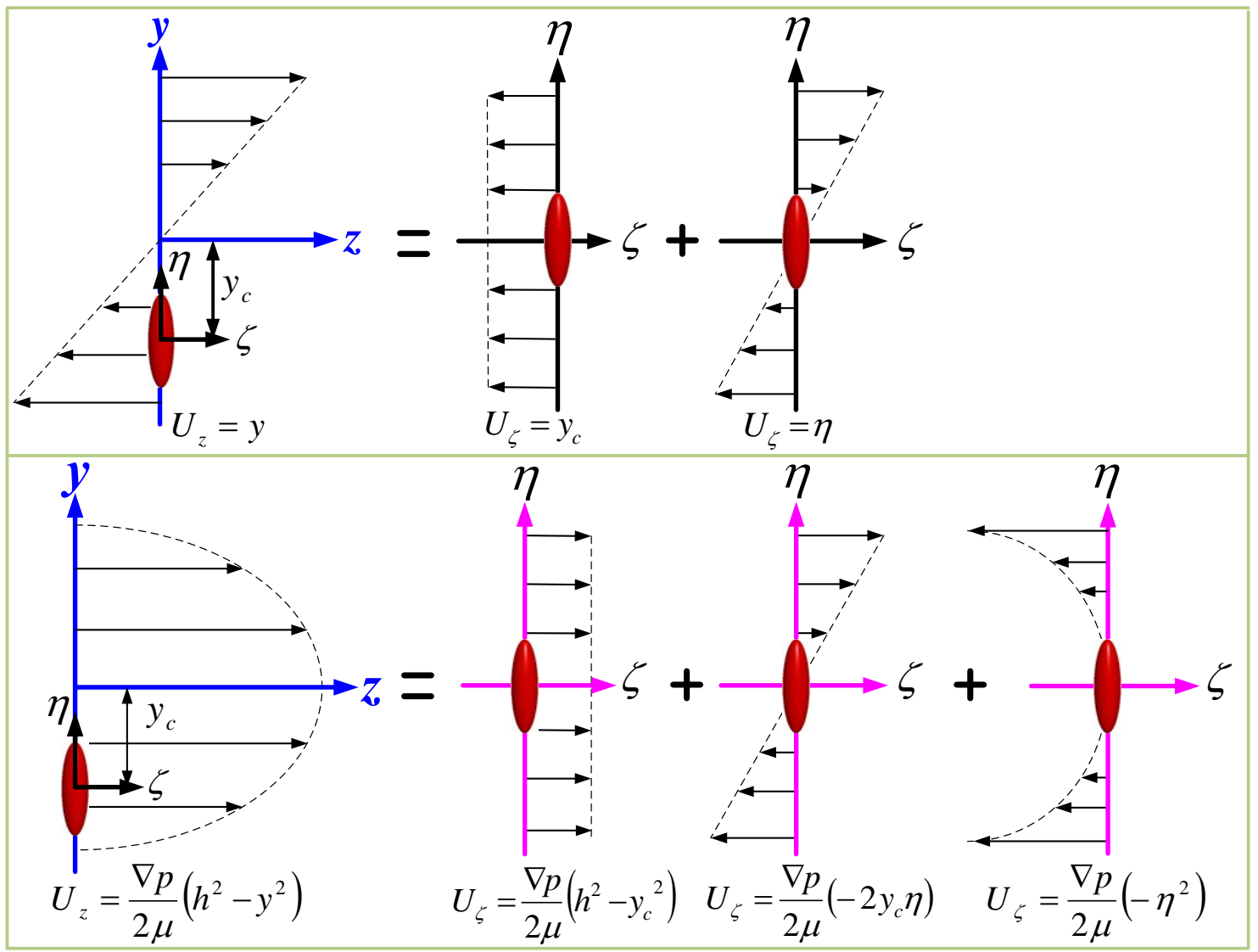

Figure 3.2: The decomposition of a simple shear flow into the $\xi \eta \zeta$ system with fiber centroid at the origin of the coordinate system: (top) A simple shear flow and (bottom) a plane Poiseuille flow.

the net force and torque vanish at every time instant. Chwang concluded that fiber orientation is still governed by Jeffery's theory, and the fiber centroid moves along a straight path with a variable speed depending on fiber orientation without the lateral motion. However, Stover [58] did not observe this variable linear motion of the fiber centroid. Feng [61] used a two-dimensional POLYFLOW solver to study a buoyant particle in Poiseuille flows and observed the Segré-Silberberg effect. In this chapter, we numerically investigate the three-dimensional fiber motion in Poiseuille flows. 


\subsection{FEM-based discrete fiber simulation}

\subsubsection{Definition of fiber orientation/position}

In the proposed FEM-based approach, there are two coordinate systems: one is the $x y z$ coordinate system, used to define the undisturbed flow field, and the other is the $x^{\prime} y^{\prime} z^{\prime}$ coordinate system that translates and rotates with the fiber, as seen in Fig.3.3. The fiber centroid is fixed at the origin of the $x^{\prime} y^{\prime} z^{\prime}$ system, where the $x^{\prime}$ axis is along the fiber's long axis and $y^{\prime}$ axis is defined by rotating vector $\hat{\boldsymbol{\theta}}$ with respect to the $x^{\prime}$ axis by $\psi$.

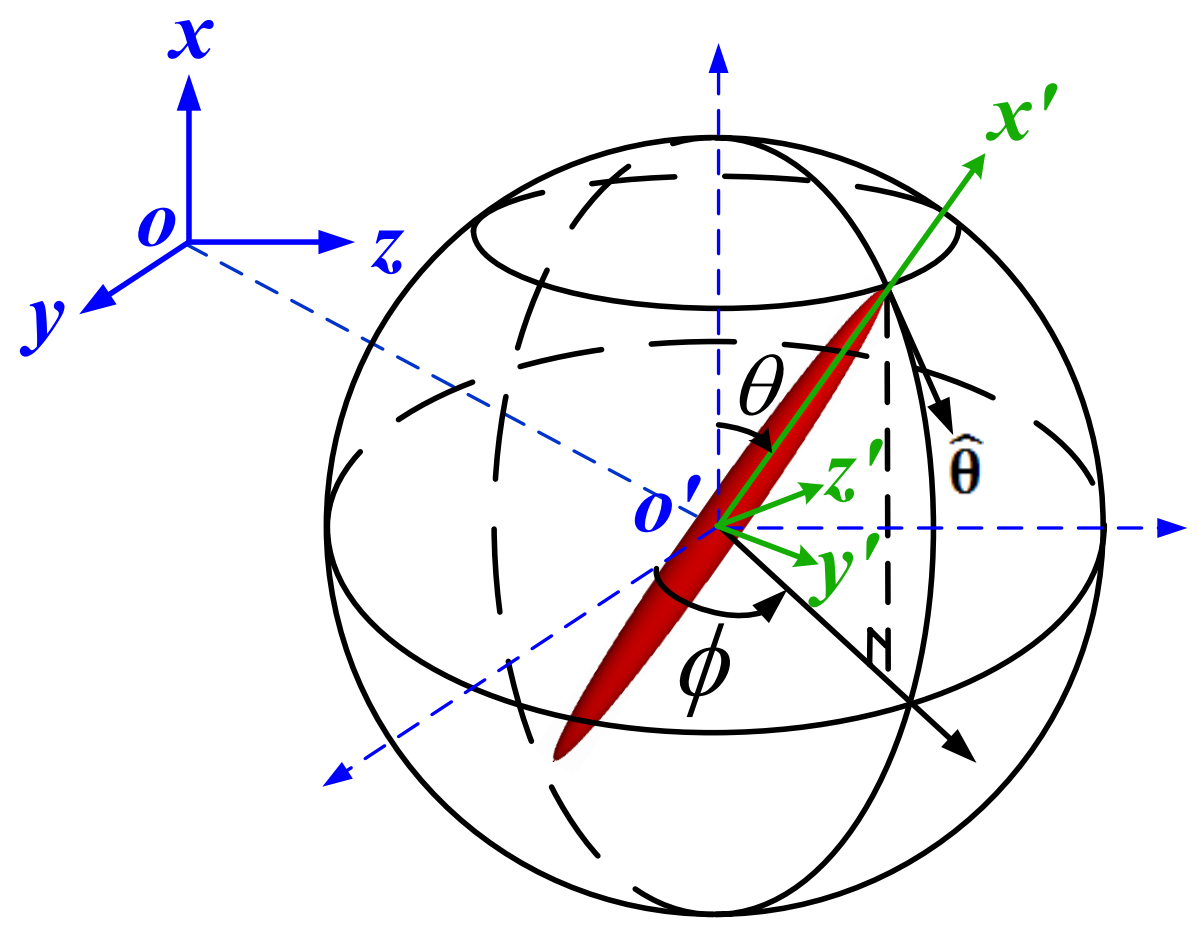

Figure 3.3: Definition of fiber orientation and position in the proposed approach

Fiber orientation is described by three angles, $\phi, \theta, \psi$, defined in the $x y z$ system 
(as seen in Fig.3.3), with the fiber's angular velocity $\boldsymbol{\omega}$ in the $x y z$ system defined as,

$$
\boldsymbol{\omega}=\left[\begin{array}{c}
\omega_{x} \\
\omega_{y} \\
\omega_{z}
\end{array}\right]=\left[\begin{array}{c}
\dot{\phi}+\dot{\psi} \cos \theta \\
-\dot{\theta} \sin \phi+\dot{\psi} \sin \theta \cos \phi \\
\dot{\theta} \cos \phi+\dot{\psi} \sin \theta \sin \phi
\end{array}\right]
$$

Fiber position is described by the position vector of the origin of the $x^{\prime} y^{\prime} z^{\prime}$ system defined in the $x y z$ system, denoted as $\left[x_{c}, y_{c}, z_{c}\right]^{T}$, with the linear velocity denoted as $\mathbf{U}_{c}=\left[\dot{x}_{c}, \dot{y}_{c}, \dot{z}_{c}\right]^{T}$.

\subsubsection{Governing equations}

The velocity and pressure distribution within the fluid is computed and then used to calculate the force and torque on the fiber. The continuity equation and isothermal incompressible Navier-Stokes equation are given, respectively, as

$$
\begin{aligned}
\nabla \cdot \mathbf{U} & =0 \\
\rho\left[\frac{\partial \mathbf{U}}{\partial t}+\mathbf{U} \cdot \nabla \mathbf{U}\right] & =-\nabla p+\mu \nabla^{2} \mathbf{U}
\end{aligned}
$$

In this research work, we consider micro and sub-micro particles, so the dimensionless Navier-Stokes equation is obtained using $L_{c}$ and $U_{c}$ to represent the characteristic length and characteristic velocity, respectively. So the characteristic time is $t_{c}=L_{c} / U_{c}$. Non-dimensionlized variables are

$$
\text { length : } L^{*}=\frac{L}{L_{c}}, \quad \text { velocity }: \mathbf{U}^{*}=\frac{\mathbf{U}}{U_{c}}, \quad \text { time }: t^{*}=\frac{t}{t_{c}}
$$

gradient operator : $\nabla^{*}=L_{c} \nabla, \quad$ laplace operator : $\nabla^{* 2}=L_{c}^{2} \nabla^{2}$

pressure $: p^{*}=\frac{p}{\mu U_{c} / L_{c}}, \quad$ force $: \mathbf{F}^{*}=\frac{\mathbf{F}}{\mu U_{c} L_{c}}, \quad$ torque : $\mathbf{T}^{*}=\frac{\mathbf{T}}{\mu U_{c} L_{c}^{2}}$ 
The dimensionless governing equations become

$$
\begin{aligned}
\nabla^{*} \cdot \mathbf{U}^{*} & =0 \\
\operatorname{Re}\left[\frac{\partial \mathbf{U}^{*}}{\partial t^{*}}+\mathbf{U}^{*} \cdot \nabla^{*} \mathbf{U}^{*}\right] & =-\nabla^{*} p^{*}+\nabla^{* 2} \mathbf{U}^{*}
\end{aligned}
$$

where the local Reynolds number $R e=\rho U_{c} L_{c} / \mu$. For low-Reynolds-number viscous flows $(R e \ll 1)$ which best represent the flow conditions for the polymeric melt within an injection mold cavity, the dimensionless governing equations are

$$
\begin{gathered}
\nabla \cdot \mathbf{U}=0 \\
-\nabla p+\nabla^{2} \mathbf{U}=0
\end{gathered}
$$

where we suppress the superscript $*$ for simplicity. Therefore, in the proposed finite element model, all parameter are dimensionless.

\subsubsection{Finite element analysis}

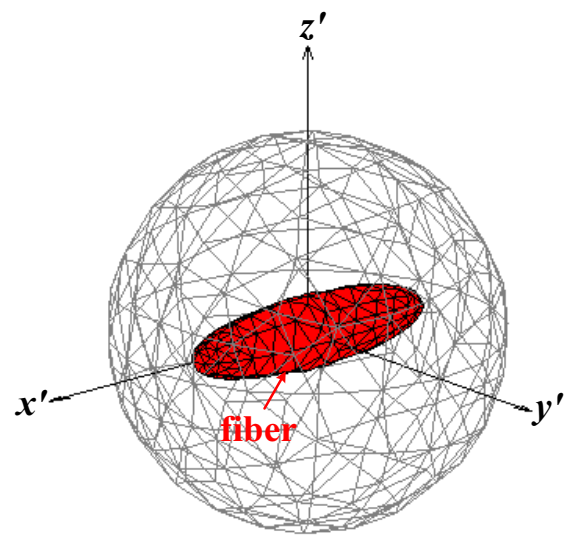

(a)

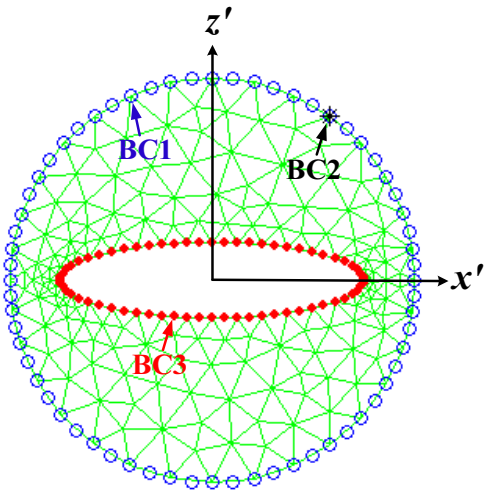

(b)



(c)

Figure 3.4: Finite element model with boundary conditions: (a) Three-dimensional finite element model with fiber centroid at the origin and long axis along the $x^{\prime}$ axis; (b) Mesh model in the plane with three applied essential boundary conditions; $(c)$ Velocity distribution of fluid domain in the plane with the applied simple shear flow. 
In the proposed finite element approach, we use tetrahedral meshes to represent the three-dimensional fluid domain between the fluid boundary (outside spheroid in Fig.3.4(a)) and the fiber (red ellipsoid in Fig.3.4(a)). Three essential/Dirichlet boundaries shown in Fig.3.4(b) are defined in the $x y z$ system as

(BC1) On fluid boundary (circles in Fig.3.4(b)):

$$
\mathbf{U}_{\mathrm{BC} 1}=\mathbf{U}_{0}
$$

(BC2) On one node of fluid boundary (one star in Fig.3.4(b)):

$$
p_{\mathrm{BC} 2}=p_{0}
$$

(BC3) On fiber surface (dots in Fig.3.4(b)):

$$
\mathrm{U}_{\mathrm{BC} 3}=\mathbf{U}_{c}+\boldsymbol{\omega} \times \mathbf{r}
$$

We apply the mixed Finite Element Method [64] to solve Eqs.(3.8)-(3.12), where the independent variables are velocity $\mathbf{U}=[u, v, w]^{T}$ and pressure $p$. The shape functions used in the proposed proposed finite element model are governed by $L B B$ compatibility condition $[64,65]$, i.e. the order of shape function for pressure should be one order lower than that used for velocity field. Appropriate shape functions are used to transform the weak form of governing equations into a system of equation $[\mathbf{K}]\{\mathbf{V}\}=\{\mathbf{F}\}$, in which $\mathbf{V}$ represents the fluid velocity and pressure at all nodes in a global sequence denoted as $\mathbf{V}=[\mathbf{U} ; p]$. The system of equations are partitioned as

$$
\left[\begin{array}{cc}
\mathbf{K}_{f f} & \mathbf{K}_{f e} \\
\mathbf{K}_{e f} & \mathbf{K}_{e e}
\end{array}\right]\left[\begin{array}{c}
\mathbf{V}_{f} \\
\mathbf{V}_{e}
\end{array}\right]=\left[\begin{array}{c}
\mathbf{F}_{f} \\
\mathbf{F}_{e}
\end{array}\right]
$$

where the subscript $f$ means the free nodal degrees of freedom with the velocity and 
pressure to be calculated, and $e$ represents the nodal degrees of freedom with the specified essential boundary conditions. It follows that velocity and pressure $\mathbf{V}_{f}$ in the fluid domain is calculated from

$$
\mathbf{K}_{f f} \mathbf{V}_{f}=\left(\mathbf{F}_{f}-\mathbf{K}_{f e} \mathbf{V}_{e}\right)
$$

The reaction force on the essential nodes (including the nodes on the fiber surface) can be calculated from

$$
\mathbf{F}_{e}=\mathbf{K}_{e f} \mathbf{V}_{f}+\mathbf{K}_{e e} \mathbf{V}_{e}
$$

The force exerted by the fluid on the fiber is obtained by summing up the reaction forces on the nodes located on the fiber surface using

$$
\mathbf{F}=-\sum_{n=1}^{N}\left(\mathbf{F}_{e}\right)_{n}
$$

where $N$ is the total number of the nodes on the fiber surface, and $n$ is the index. In a similar manner, the torque on the fiber is obtained by summing up the cross product of position vector $\mathbf{r}$ and reaction force $\mathbf{F}_{e}$ at the nodes located on the fiber surface as

$$
\mathbf{T}=-\sum_{n=1}^{N}\left(\mathbf{r} \times \mathbf{F}_{e}\right)_{n}
$$

\subsubsection{Searching fiber velocities}

In the proposed finite element model, fiber velocities $\mathbf{U}_{c}$ and $\boldsymbol{\omega}$ define the boundary condition BC3 (cf. Eq.(3.12)) when solving for $\mathbf{U}$ and $p$ within the fluid, and also the force $\mathbf{F}$ and torque $\mathbf{T}$ exerted on fiber. Therefore, $\mathbf{F}$ and $\mathbf{T}$ are functions of $\dot{x}_{c}, \dot{y}_{c}, \dot{z}_{c}$

and $\dot{\phi}, \dot{\theta}, \dot{\psi}$. As in Jeffery's original work, the force and torque exerted on the massless 
fiber are set to zero at any time instant $t_{i}$ as

$$
\begin{aligned}
& \mathbf{F}\left(\dot{x}_{c i}, \dot{y}_{c i}, \dot{z}_{c i}, \dot{\phi}_{i}, \dot{\theta}_{i}, \dot{\psi}_{i}\right)=\mathbf{0} \\
& \mathbf{T}\left(\dot{x}_{c i}, \dot{y}_{c i}, \dot{z}_{c i}, \dot{\phi}_{i}, \dot{\theta}_{i}, \dot{\psi}_{i}\right)=\mathbf{0}
\end{aligned}
$$

using the Newton-Raphson iteration algorithm to compute the fiber's linear and angular velocities at any time $t_{i}$. This is done by solving Eqs.(3.18) and (3.19) simultaneously as $\dot{\mathbf{y}}_{I+1}=\dot{\mathbf{y}}_{I}-\left[\mathbf{J}_{I}\right]^{-1}\left[\mathbf{Q}_{I}\right]$, where $I$ is the Newton-Raphson iteration number, $\dot{\mathbf{y}}_{I}=\left[\dot{x}_{c i}, \dot{y}_{c i}, \dot{z}_{c i}, \dot{\phi}_{i}, \dot{\theta}_{i}, \dot{\psi}_{i}\right]^{T}$, and $\left[\mathbf{Q}_{I}\right]=[\mathbf{F} ; \mathbf{T}]$, which is a function of $\mathbf{F}_{e}$. In this analysis, $\left[\mathbf{J}_{I}\right]$ is the Jacobian matrix (i.e., the first-order partial derivatives of $\left[\mathbf{Q}_{I}\right]$ with respect to $\left.\dot{\mathbf{y}}_{I}\right)$, shown as

$$
\frac{\partial \mathbf{Q}_{I}}{\partial \dot{\mathbf{y}}_{I}}=\left[\frac{\partial \mathbf{F}}{\partial \dot{\mathbf{y}}_{I}} ; \frac{\partial \mathbf{T}}{\partial \dot{\mathbf{y}}_{I}}\right]=\left[-\sum_{n=1}^{N} \frac{\partial\left(\mathbf{F}_{\mathbf{e}}\right)_{n}}{\partial \dot{\mathbf{y}}_{I}} ;-\sum_{n=1}^{N}\left(\mathbf{r}_{e}\right)_{n} \times \frac{\partial\left(\mathbf{F}_{\mathbf{e}}\right)_{n}}{\partial \dot{\mathbf{y}}_{I}}\right]
$$

in which $\partial \mathbf{F}_{\mathbf{e}} / \partial \dot{\mathbf{y}}_{I}$ is evaluated as

$$
\frac{\partial \mathbf{F}_{e}}{\partial \dot{\mathbf{y}}_{I}}=\frac{\partial \mathbf{K}_{e f}}{\partial \dot{\mathbf{y}}_{I}} \mathbf{V}_{f}+\mathbf{K}_{e f} \frac{\partial \mathbf{V}_{f}}{\partial \dot{\mathbf{y}}_{I}}+\frac{\partial \mathbf{K}_{e e}}{\partial \dot{\mathbf{y}}_{I}} \mathbf{V}_{e}+\mathbf{K}_{e e} \frac{\partial \mathbf{V}_{e}}{\partial \dot{\mathbf{y}}_{I}}
$$

For a Newtonian fluid, the stiffness matrix $\mathbf{K}$ is independent of $\dot{\mathbf{y}}_{I}$, and $\partial \mathbf{V}_{e} / \partial \dot{\mathbf{y}}_{I}$ is prescribed as the partial derivative of essential boundary condition with respect to fiber velocities. The only unknown in Eq.(3.21) is $\partial \mathbf{V}_{f} / \partial \dot{\mathbf{y}}_{I}$, calculated from

$$
\mathbf{K}_{f f} \frac{\partial \mathbf{V}_{f}}{\partial \dot{\mathbf{y}}_{I}}=\left(\frac{\partial \mathbf{F}_{f}}{\partial \dot{\mathbf{y}}_{I}}-\mathbf{K}_{f e} \frac{\partial \mathbf{V}_{e}}{\partial \dot{\mathbf{y}}_{I}}\right)
$$

Therefore, at any time instant $t_{i}$, with the given fiber position $x_{c i}, y_{c i}, z_{c i}$ and orientation $\phi_{i}, \theta_{i}, \psi_{i}$, the linear velocity $\dot{x}_{c i}, \dot{y}_{c i}, \dot{z}_{c i}$ and angular velocity (defined by $\dot{\phi}_{i}, \dot{\theta}_{i}, \dot{\psi}_{i}$ ) are obtained from the converged Newton-Raphson iteration to give $\dot{\mathbf{y}}_{i}=\mathbf{f}\left(t_{i}, \mathbf{y}_{i}\right)$, where $\mathbf{y}_{i}=\left[x_{c i}, y_{c i}, z_{c i}, \phi_{i}, \theta_{i}, \psi_{i}\right]^{T}$. 


\subsubsection{Tracking fiber motion with transformed boundary}

A $4^{\text {th }}$-order Runge-Kutta algorithm is used to solve the evolution of $x_{c}, y_{c}, z_{c}, \phi, \theta, \psi$ as a function of time, with the given initial conditions $\mathbf{y}_{0}=\left[x_{c 0}, y_{c 0}, z_{c 0}, \phi_{0}, \theta_{0}, \psi_{0}\right]^{T}$, as seen in Eq.(2.14). The relative position and orientation of fiber with respect to the fluid boundary keep changing, because fiber rotates and translates relative to the fixed fluid boundary. The straight-forward approach is to re-mesh the fluid domain and then update the stiffness matrix in different time instants. However, the re-meshing procedure is tedious and time-consuming.

In order to avoid the re-meshing process, the proposed finite element model is solved in the local $x^{\prime} y^{\prime} z^{\prime}$ coordinate system, in which fiber is always fixed in space with the centroid located at the origin $o^{\prime}$ and the long axis along the $x^{\prime}$ axis. The unchanged essential boundary condition on fluid boundary $\mathbf{U}_{\mathrm{BC} 1}$ is originally defined in the $x y z$ system, but is transformed into the local $x^{\prime} y^{\prime} z^{\prime}$ system according to fiber orientation (defined by $\phi, \theta, \psi)$ and position $\left(x_{c}, y_{c}, z_{c}\right)$ at current time instant $t_{i}$. The transformed boundary condition $\mathbf{U}_{0}^{\prime}$ in the $x^{\prime} y^{\prime} z^{\prime}$ system is shown as

$$
\mathbf{U}_{\mathrm{BC} 1}^{\prime}=\mathbf{U}_{0}^{\prime}=\mathbf{R} \mathbf{U}_{0}\left(x^{\prime}+x_{c}, y^{\prime}+y_{c}, z^{\prime}+z_{c}\right)
$$

where $\mathbf{U}_{0}$ is the undisturbed flow field given in the $x y z$ system, and $\mathbf{R}$ is the transformation matrix between two coordinate systems, defined as

$$
\mathbf{R}=\left[\begin{array}{ccc}
l_{1} & l_{2} & l_{3} \\
m_{1} & m_{2} & m_{3} \\
n_{1} & n_{2} & n_{3}
\end{array}\right]
$$


In the above,

$$
\begin{aligned}
& l_{1}=\cos \theta, \quad l_{2}=-\sin \theta \cos \psi, \quad l_{3}=\sin \theta \sin \psi, \\
& m_{1}=\sin \theta \cos \phi, \quad n_{1}=\sin \theta \sin \phi \\
& m_{2}=-\sin \phi \sin \psi+\cos \theta \cos \phi \cos \psi, \quad n_{2}=\cos \phi \sin \psi+\cos \theta \sin \phi \cos \psi \\
& m_{3}=-\sin \phi \cos \psi-\cos \theta \cos \phi \sin \psi, \quad n_{3}=\cos \phi \cos \psi-\cos \theta \sin \phi \sin \psi
\end{aligned}
$$

Therefore, in the proposed finite element approach, the stiffness matrix $\mathbf{K}$ remains unchanged, but the applied essential boundary condition on the fluid boundary keeps updating as fiber moves, which tremendously reduces the computation time. Since the finite element model is solved in the $x^{\prime} y^{\prime} z^{\prime}$ system, the essential boundary conditions $\mathbf{U}_{\mathrm{BC} 3}$ are also transformed into the local $x^{\prime} y^{\prime} z^{\prime}$ system, defined as

$$
\mathbf{U}^{\prime}{ }_{\mathrm{BC} 3}=\mathbf{U}_{c}^{\prime}+\boldsymbol{\omega}^{\prime} \times \mathbf{r}_{e}^{\prime}
$$

where $\mathbf{U}^{\prime}$ and $\boldsymbol{\omega}^{\prime}$ are fiber's linear velocity and angular velocity, respectively, defined as

$$
\mathbf{U}_{c}^{\prime}=\left[\dot{x}_{c}^{\prime}, \dot{y}_{c}^{\prime}, \dot{z}_{c}^{\prime}\right]^{T}=\mathbf{R}^{T}\left[\dot{x}_{c}, \dot{y}_{c}, \dot{z}_{c}\right]^{T}
$$

and

$$
\boldsymbol{\omega}^{\prime}=\left[\begin{array}{c}
\omega_{x}^{\prime} \\
\omega_{y}^{\prime} \\
\omega_{z}^{\prime}
\end{array}\right]=\left[\begin{array}{c}
\dot{\phi} \cos \theta+\dot{\psi} \\
\dot{\theta} \sin \psi-\dot{\phi} \sin \theta \cos \psi \\
\dot{\theta} \cos \psi+\dot{\phi} \sin \theta \sin \psi
\end{array}\right]
$$

where the superscript ' indicates that the parameter is defined in the $x^{\prime} y^{\prime} z^{\prime}$ system. Therefore, in the proposed approach, the mesh model and stiffness matrix $\mathbf{K}$ keep unchanged as fiber moves. As seen in Eqs.(3.14) and (3.22), $\mathbf{K}$ is used to solve the system of equations for different fiber orientations and positions. Since $\mathbf{K}$ is a constant, which is decomposed using the LU factorization to save the computation 
time in the proposed approach.

\subsubsection{Summary of the proposed methodology}

The proposed FEM-based simulation approach uses the mixed finite element model. The overall process is summarized as follows:

STEP 1: The finite element model is set up to describe the fluid domain between fiber surface and the fluid boundary far away from the fiber. At $t_{i}(i=0)$, fiber orientation (defined by $\phi_{0}, \theta_{0}$ and $\left.\psi_{0}\right)$ and position $\left(x_{c 0}, y_{c 0}, z_{c 0}\right)$ are given, known as the initial condition. Fiber orientation and position are used to define the boundary condition $\mathrm{BC} 1$ of the finite element model.

STEP 2: At any time instant $t_{i}$, with the current fiber orientation and position, the boundary condition on the fluid boundary is updated. Then fiber velocities $\left(\dot{x}_{c i}, \dot{y}_{c i}, \dot{z}_{c i}\right.$

and $\left.\dot{\phi}_{i}, \dot{\theta}_{i}, \dot{\psi}_{i}\right)$ can be obtained using the Newton-Raphson method with the analytical Jacobian matrix.

STEP 3: At $t_{i+1}$, fiber orientation and position are updated from the fiber velocities at the time instant $t_{i}$ using a $4^{\text {th }}$-order Runge-Kutta algorithm. Then the time counter is set to be $i=i+1$ and the process goes to STEP 2 .

\subsection{Implementation and examples}

In this section, fiber motions in various homogeneous and inhomogeneous flows are investigated and compared with those in available theories and experiments where possible. 


\subsubsection{Validation of the proposed methodology}

Our computational methodology is validated by evaluating the motion of a single ellipsoidal fiber in a simple shear flow, and comparing our results with Jeffery's analytical solution. Dimensionless parameters for this study are tabulated in Table 3.1, where $a$ and $b$ are the long and short axes of an ellipsoidal fiber, respectively, and $R$ is the radius of the spheroidal computational domain. In this chapter, we study the fiber motion in a unbounded fluid domain. From our simulation results, when the ratio of the size of fluid domain to fiber size is greater than 20, the effect of bounded wall can be neglected. In this study, we use $2 R / a=40$ for all simulations.

\begin{tabular}{|c|c|}
\hline Parameters & Value \\
\hline \hline Fiber shape and aspect ratio & $a=3, b=1, r_{e}=a / b=3$ \\
\hline Simple shear flow & $U_{x}=U_{y}=0, U_{z}=\dot{\gamma} y$, where $\dot{\gamma}=1$ \\
\hline Initial fiber orientation & $\phi=0, \theta_{0}=\pi / 3, \psi_{0}=0$ \\
\hline Initial fiber centroid position & $x_{c 0}=0, y_{c 0}=1, z_{c 0}=0$ \\
\hline
\end{tabular}

Table 3.1: Dimensionless parameters to simulate an ellipsoidal fiber motion

The motion of a single ellipsoidal fiber using the proposed methodology is compared with Jeffery's solution in Fig.3.5. In this example, we apply $\Delta t=0.2$ in the Runge-Kutta method. The initial $y_{c 0}$ equals 1 and the initial linear velocity of the fiber centroid in the $z$ direction is $\dot{z}_{c 0}=\dot{\gamma} y_{c 0}=1$. Figure 3.5(a) shows that fiber orientation agrees with Jeffery's equation and Fig.3.5(b) illustrates that fiber centroid translates with the same linear velocity as the undisturbed simple shear flow evaluated at fiber centroid. The comparison of two sets of data, i.e., Jeffery's data and the numerical data, appears in Table 3.2 .

\begin{tabular}{|c|r|r|}
\hline Data Sets & $E_{R}$ & $E_{A}$ \\
\hline \hline$\phi$ & $5.2 E-5$ & 0.009 \\
\hline$\theta$ & $2.8 E-5$ & 0.002 \\
\hline$\psi$ & $8.3 E-5$ & 0.001 \\
\hline
\end{tabular}

Table 3.2: Comparison of Jeffery's data and the numerical data 

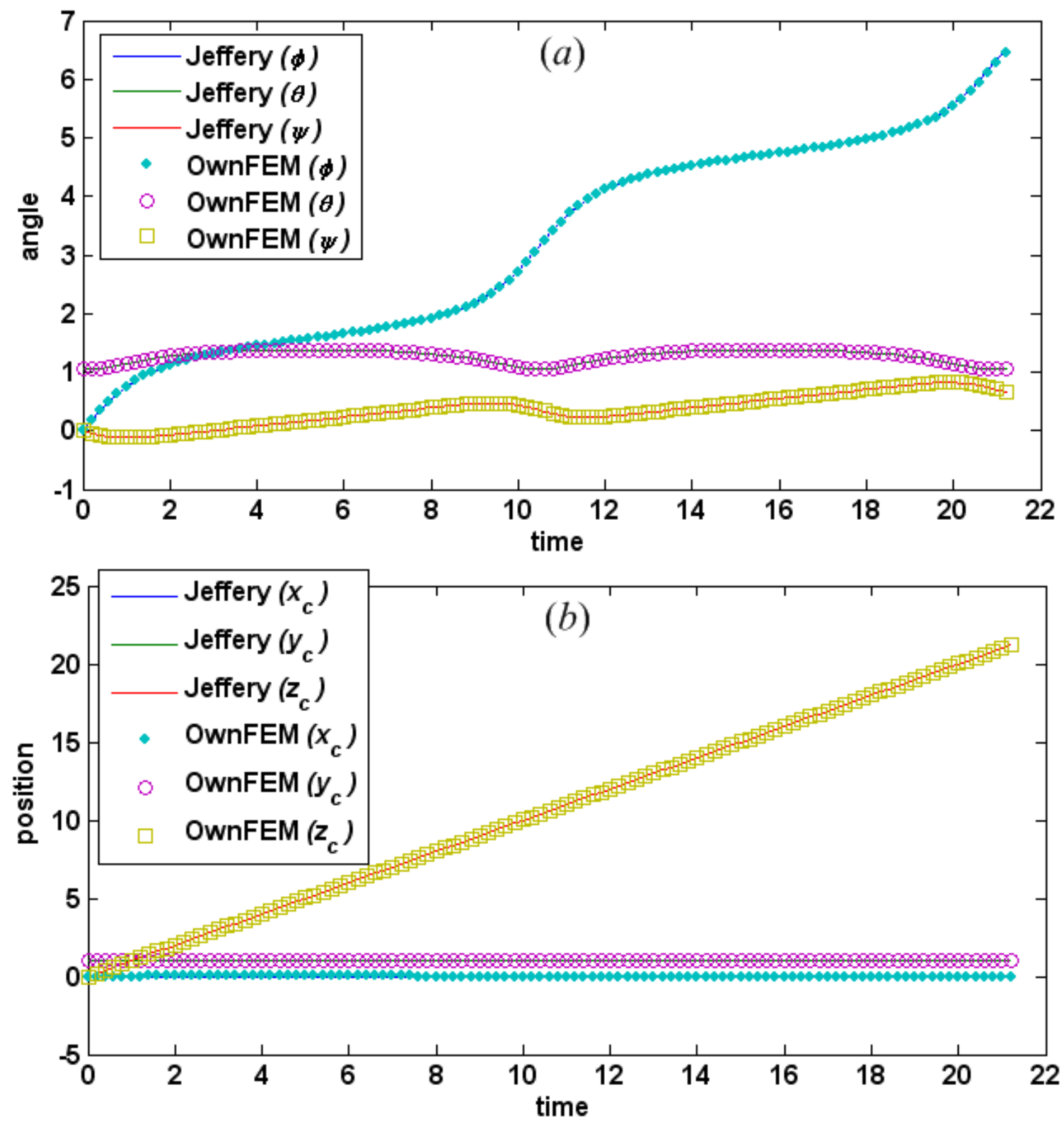

Figure 3.5: Numerical results of three-dimensional ellipsoidal fiber motion in one complete period: $(a)$ fiber orientation defined by $\phi, \theta, \psi$ and $(b)$ fiber position defined by $x_{c}, y_{c}, z_{c}$

The excellent agreement between Jeffery's theory and our approach validates the accuracy and robustness of our approach. In this finite element model, there are 29228 nodes, 19509 elements and 91762 degrees of freedom. The simulation is implemented using the computer with four $2 \mathrm{GHz}$ CPU cores and $32 \mathrm{~GB}$ RAM. The time efficiency of calculation is listed in Table 3.3 and compared with the commercial software 
COMSOL multiphysics using the finite difference derivative in the Newton-Raphson method. In COMSOL multiphysics, the Navier-Stokes equation is used, but the parameters are set up to guarantee the low-Reynolds-number condition. The Jacobian matrix is obtained using the finite difference method, which requires the multiple calling of COMSOL finite element solver. By contrast, the proposed methodology solves pure Stokes equation directly with the analytical Jacobian matrix obtained from our finite element model. LU factorization is used to decompose the stiffness matrix once at the beginning of the calculation, and saved to solve the system of equations. The computation efficiency is tremendously improved, as seen in Table 3.3.

\begin{tabular}{|c|c|c|}
\hline Solver & Proposed FEM package & COMSOL multiphysics \\
\hline \hline $\begin{array}{c}\text { Governing } \\
\text { equation }\end{array}$ & Pure Stokes equation & $\begin{array}{c}\text { Navier-Stokes with } \\
\text { low Reynolds number }\end{array}$ \\
\hline Derivative calculation & Analytical Jacobian & finite difference derivative \\
\hline Solver & LU factorization & Iterative method \\
\hline Time efficiency & 1.77 hours & 80 hours $(>3$ days $)$ \\
\hline $\begin{array}{c}\text { No. of steps } \\
\text { in Runge-Kutta }\end{array}$ & 107 steps & 107 steps \\
\hline
\end{tabular}

Table 3.3: Efficiency of the calculation of the proposed FEM package

\subsubsection{Fiber motion in various homogeneous flows}

Simple shear flow is one of homogeneous flows with a constant velocity gradient of the undisturbed flow field. In this section, the motion of a single fiber in various homogeneous flows is solved using the proposed finite element approach, and compared with Jeffery's results $[10,11]$. 


\section{Rotational flow}

The dimensionless undisturbed rotational flow field is defined in the $x y z$ system as

$$
\mathbf{U}_{\mathrm{BC} 1}=\left[\begin{array}{ccc}
0 & 0 & 0 \\
0 & 0 & -1 \\
0 & 1 & 0
\end{array}\right]\left[\begin{array}{l}
x \\
y \\
z
\end{array}\right]
$$

Then the corresponding vorticity tensor and strain rate tensor are calculated as

$$
\boldsymbol{\Omega}=\left[\begin{array}{ccc}
0 & 0 & 0 \\
0 & 0 & 1 \\
0 & -1 & 0
\end{array}\right]
$$

and

$$
\Gamma=0
$$

It can be seen that fiber rotation in a pure rotational flow results from the vorticity tensor, while shear rate tensor does have any effect on fiber rotation. With the given initial fiber orientation $\mathbf{P}_{\mathbf{0}}$, the fiber orientation $\mathbf{P}$ can be calculated using Eq.(1.6), which is used to check the proposed approach. The dimensionless simulation parameters are defined as follows: fiber's geometric aspect ratio $r_{e}=3$, initial orientation $\phi=0, \theta=\pi / 3, \psi=0$, i.e., $\mathbf{P}_{0}=[0.5,0.75,0.433]^{T}$. The plot of fiber orientation defined by the unit vector $\mathbf{P}=[\mathrm{P} 1, \mathrm{P} 2, \mathrm{P} 3]^{T}$ is shown in Fig.3.6, from which we can see the results obtained from the proposed simulation tool are in excellent agreement with Jeffery's equation. 

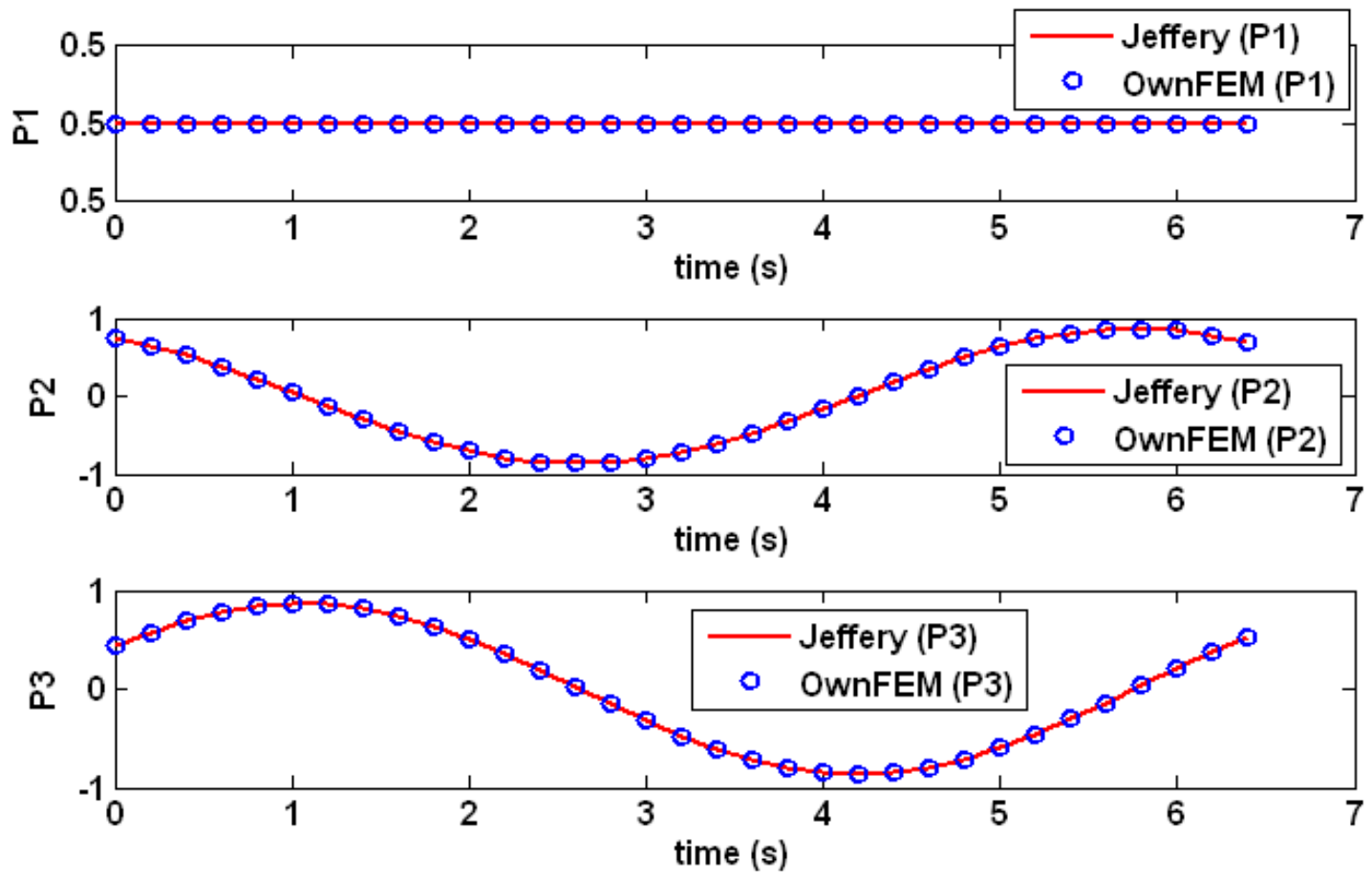

Figure 3.6: Fiber orientation in a rotational flow

\section{Stretching flow}

In a similar manner, the dimensionless stretching flow field in the global $x y z$ system is defined as

$$
\mathbf{U}_{\mathrm{BC} 1}=\left[\begin{array}{ccc}
0 & 0 & 0 \\
0 & -1 & 0 \\
0 & 0 & 1
\end{array}\right]\left[\begin{array}{l}
x \\
y \\
z
\end{array}\right]
$$

Then the vorticity tensor and strain rate tensor are calculated as

$$
\Omega=0
$$

and

$$
\boldsymbol{\Gamma}=\left[\begin{array}{ccc}
0 & 0 & 0 \\
0 & -1 & 0 \\
0 & 0 & 1
\end{array}\right]
$$

In this case, the vorticity tensor of fluid field is zero. So fiber motion only results from 
the strain rate tensor, which makes fiber align with flow direction permanently. The plot of fiber orientation is shown in Fig.3.7 using the same simulation parameters as those in the rotational flow. It can be seen that the numerical data from the proposed finite element package is in excellent agreement with Jeffery's theory, which also validates the proposed methodology. In the next section, the proposed FEM package is used to solve the fiber motion in inhomogeneous flows, which is beyond the scope of Jeffery's paper [8].
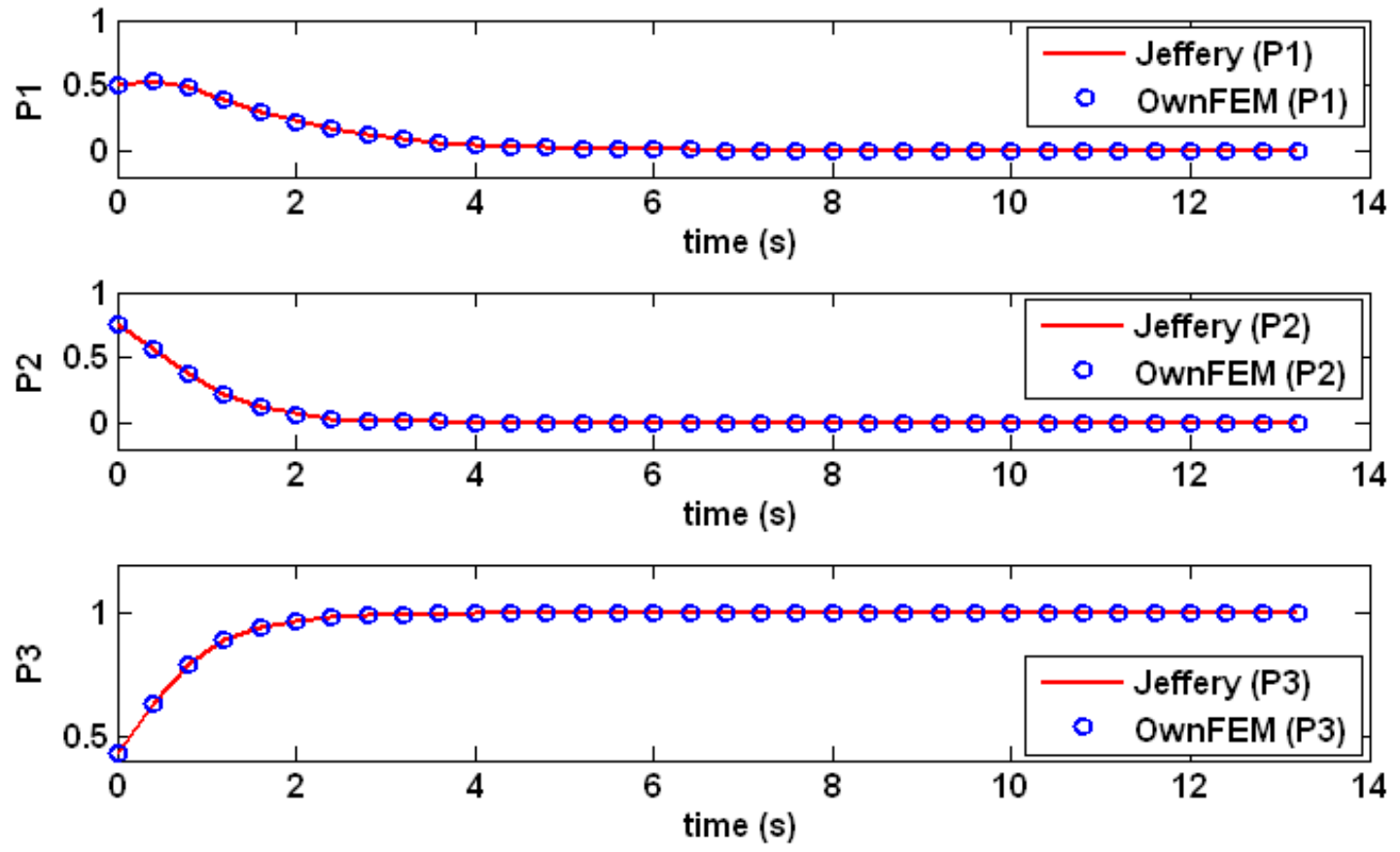

Figure 3.7: Fiber orientation in a stretching flow

\subsubsection{Fiber motion in Poiseuille flows}

Inhomogeneous flows are defined as the flows where the velocity gradient is a function of spatial coordinates. In this section, we investigate the fiber motion in a plane Poiseuille flow and a circular Poiseuille flow. The study of fiber suspensions in Poiseuille flows is critical, since the thermoset resin in a mold cavity is treated as a pressure-driven flow or Poiseuille flow. 


\section{Plane Poiseuille flow}

Plane Poiseuille flow is a pressure-driven flow between two parallel flat plates with zero velocity on two plates that forces the boundaries of the flow domain, as seen in Fig.3.1(a). Fibers traveling near the upper and lower walls are influenced by the fixed boundary, which is discussed in Chapter 5. In this section, we study the unbounded plane Poiseuille flow, which means that the fluid domain goes beyond the two plates $(R>>h)$, with the velocity boundary condition (cf.Eq.(3.1)) prescribed on the faraway fluid boundary. As a result, we do not consider the boundary effect in this study and focus on the dynamic behavior of suspended fibers in a plane Poiseuille flow. The velocity profile of the plane Poiseuille flow in the global $x y z$ system is given in Sec.3.2. By using the characteristic length $L_{c}$ and velocity $U_{c}$, the dimensionless velocity profile with the suppressed $*$ is given as $U_{x}=U_{y}=0, U_{z}=\frac{\nabla p}{2}\left(h^{2}-y^{2}\right)$, where $h$ is the dimensionless half distance between two plates, and $\nabla p$ is the dimensionless pressure gradient in the $z$ direction. The flow field can be decomposed into three flow components in the $\xi \eta \zeta$ system (as seen in Fig.3.2) with the given position of fiber centroid in the $x y z$ system defined as $\left[x_{c}, y_{c}, z_{c}\right]^{T}$. The three flow components in the $\xi \eta \zeta$ coordinate system are shown as

uniform flow component:

$$
U_{\zeta}=\frac{\nabla p}{2}\left(h^{2}-y_{c}^{2}\right)
$$

shear flow component:

$$
U_{\zeta}=\frac{\nabla p}{2}\left(-2 y_{c} \eta\right)
$$

and quadratic flow component:

$$
U_{\zeta}=\frac{\nabla p}{2}\left(-\eta^{2}\right)
$$

in which the uniform flow component and quadratic flow component cause the linear 
movement of fiber, while shear flow component results in the fiber rotation. It can be seen that all three flow components have the dimensionless constant coefficient $\frac{\nabla p}{2}$.

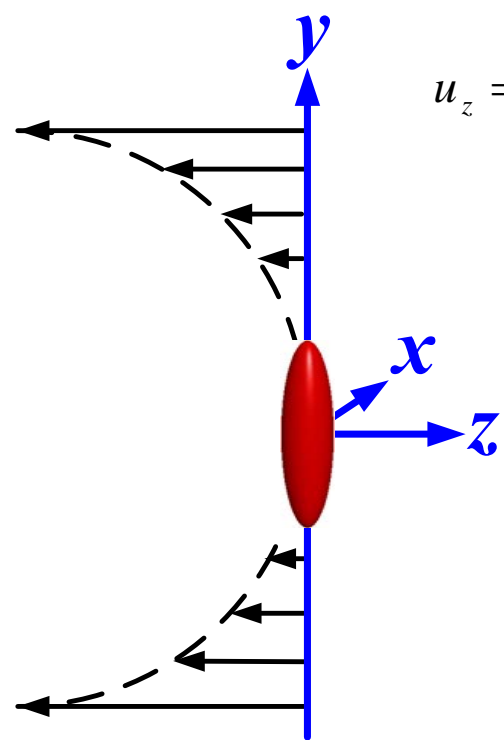

(a)

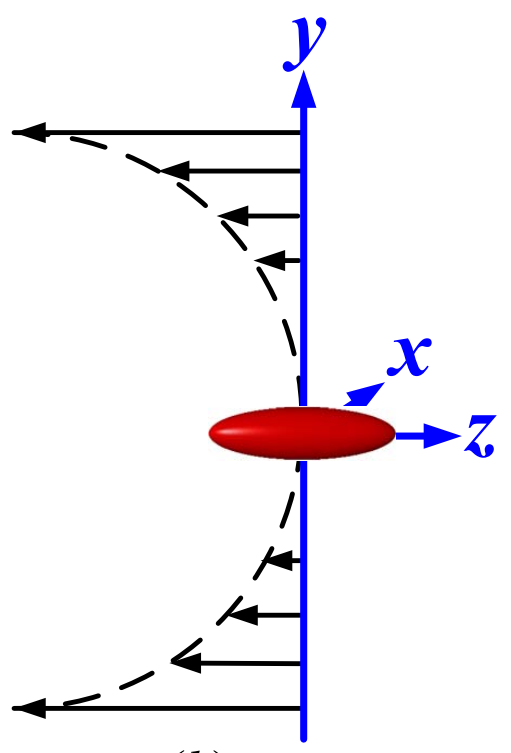

(b)

Figure 3.8: Fiber motion in a quadratic flow component with different initial orientations: ( $a$ ) fiber in the vertical direction (case 1) and (b) fiber in the horizontal direction (case 2)

First, we investigate the linear motion of fiber in the sole quadratic flow component with fiber centroid located at the centerline of fluid flow. We defined the undisturbed flow field as $U_{x}=U_{y}=0, U_{z}=\frac{\nabla p}{2}\left(-y^{2}\right)$ in the $x y z$ coordinate system. In order to simplify the study, we choose the coefficient $\frac{\nabla p}{2}$ to be 1 in this simulation, and then investigate the effect of pressure gradient. Therefore, the boundary condition on the far-away fluid boundary is $\mathbf{U}_{\mathrm{BC} 1}=\left[0,0,-y^{2}\right]^{T}$. We present two examples: in one example, fiber initially lies in the vertical direction, while in the other example, fiber initially lies along the horizontal direction, with the configuration shown in Fig.3.8. The initial condition of fiber motion in case 1 (cf.Fig. 3.8(a)) is $\phi=0, \theta=\pi / 2, \psi=0$ and $x_{c}=y_{c}=z_{c}=0$, and in case 2 (cf.Fig.3.8(b)) is $\phi=\pi / 2, \theta=\pi / 2, \psi=0$ and $x_{c}=y_{c}=z_{c}=0$. We consider an ellipsoid-shaped fiber with an aspect ratio of 3 , and the fluid domain is 40 times larger than the fiber size, i.e. $2 R / a=40$, so the 
effect of fluid domain is trivial, which can be seen as the unbounded problem. From our results, fiber orientations remain unchanged in both cases because fibers initially locate at the centerline of the quadratic flow component. In case 1, fiber remains vertical, while in case 2 , fiber remains horizontal throughout the flow solution.

Fiber's linear velocities in both cases are shown in Fig.3.9, which shows that even though the fluid velocity at fiber centroid is zero, the fiber itself translates backwards, because the non-zero quadratic flow component creates a non-zero force in the negative $z$ direction, which drags the fiber backwards. In Fig.3.9(a), fiber's linear velocity in the $z$ direction is about -0.75 when the fiber's long axis is perpendicular to the fluid flow, while in Fig.3.9(b), fiber's linear velocity is -0.1 when the fiber aligns along the flow direction. Therefore, in pure quadratic flow component, a fiber does not have the same linear velocity as the undisturbed quadratic flow evaluated at fiber centroid. More importantly, we can see that fiber's linear velocity relies on its orientation. The change of fiber's linear velocity with the vertical orientation is larger than that with the aligned orientation.

In this example, $\frac{\nabla p}{2}=1$, and the linear velocity is about -0.75 when the fiber remains vertical. Choosing different $\nabla p$ could amplify or reduce the change of fiber's linear velocity. Next, we investigate the effect of $\nabla p$ on the change of fiber's linear velocities, as seen in Fig.3.10, from which it can be seen that the change of fiber's linear velocity in the $z$ direction is proportional to the dimensionless pressure gradient $\nabla p$. In Stover's experiments [58], the variation of fiber's linear velocity is not observed, because the dimensionless pressure gradient could be tiny in the experiment.

Next, we investigate fiber orientation and the change of fiber's linear velocity as fiber moves in a general plane Poiseuille flow considering the three flow components together, as seen in Eqs.(3.30-3.32). In this example, the essential boundary condition 



Figure 3.9: Fiber's linear motion in a quadratic flow component: (a) fiber's linear velocity in case 1 and (b) fiber's linear velocity in case 2 . 


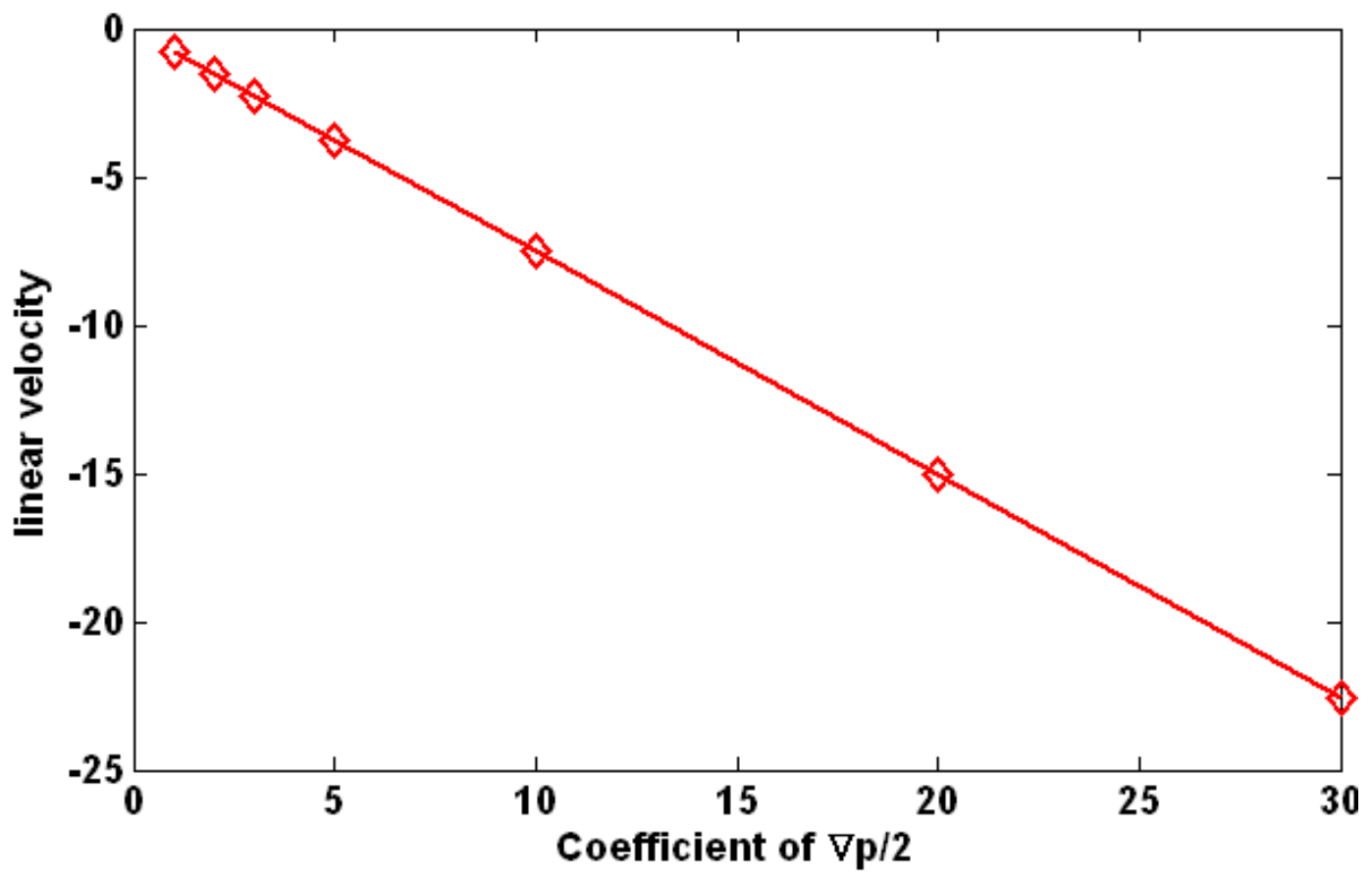

Figure 3.10: Relationship between the coefficient $\nabla p / 2$ and fiber's linear velocity in the $z$ direction

$\mathrm{U}_{\mathrm{BC} 1}$ in the $x y z$ system is

$$
\mathbf{U}_{\mathrm{BC} 1}=\left[0,0, \frac{\nabla p}{2}\left(h^{2}-y^{2}\right)\right]^{T}
$$

where we assume $h=8$ and $\frac{\nabla p}{2}=1$. The initial condition of fiber is $\phi=0, \theta=$ $\pi / 3, \psi=0$ and $x_{c}=0, y_{c}=-0.5, z_{c}=0$. Therefore, the shear rate of the undisturbed quadratic flow evaluated at fiber centroid is 1 initially.

Fiber orientation is shown in Fig.3.11, from which it can be seen that fiber rotation is governed by Jeffery's equation with the shear rate evaluated at fiber centroid. Fiber's linear velocity in the $z$ direction is shown in Fig.3.12, from which it can be seen that fiber moves along the flow field with various linear velocities, which depends on fiber orientation. When fiber's long axis is perpendicular to the fluid flow $(\phi=0)$, the difference between fiber's linear velocity and fluid velocity is larger compared with that when fiber aligns in the flow direction $(\phi=\pi / 2)$. The numerical results confirm 

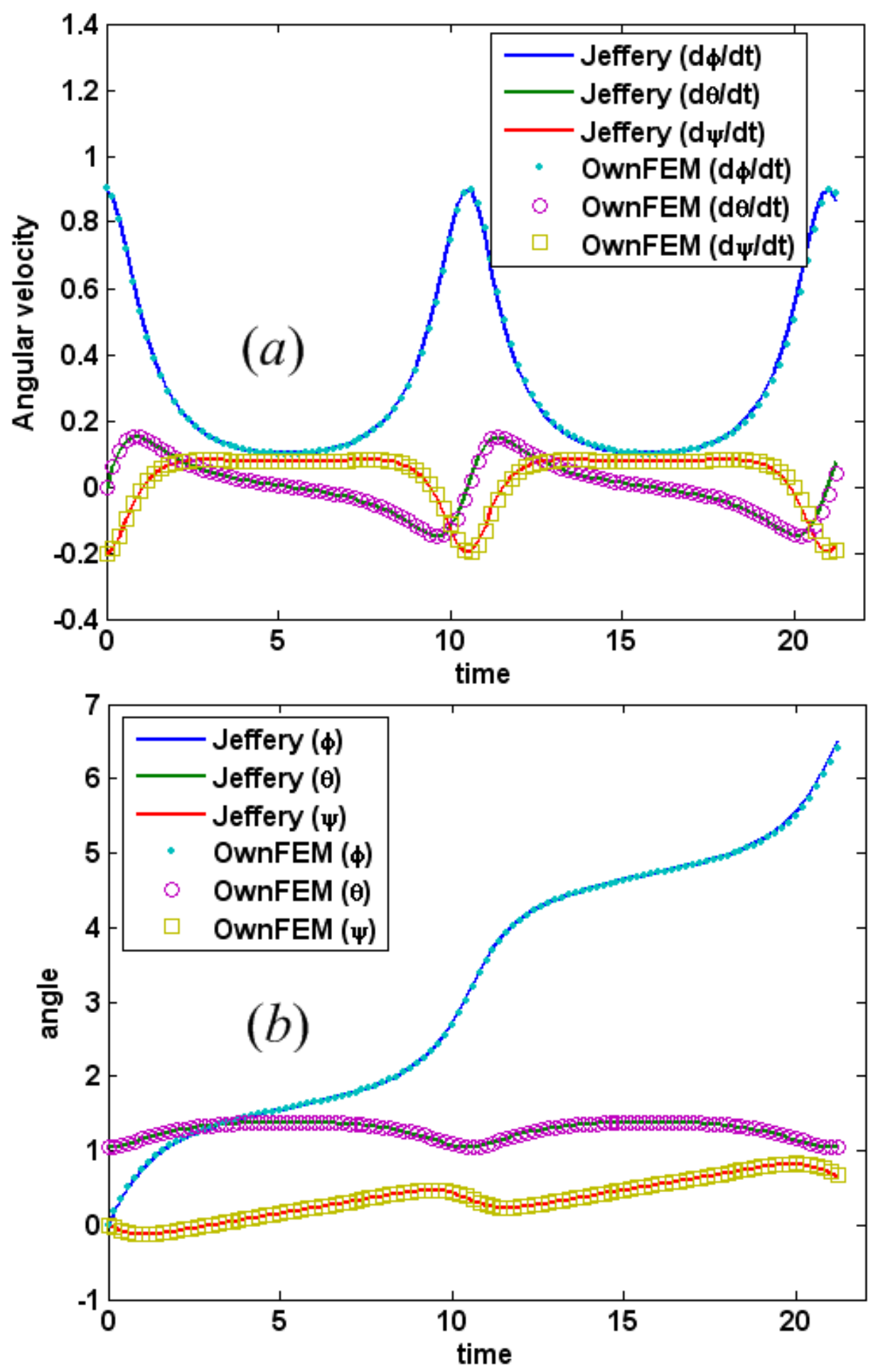

Figure 3.11: Fiber rotation in a general plane Poiseuille flow: (a) fiber's angular velocity and (b) fiber orientation. 

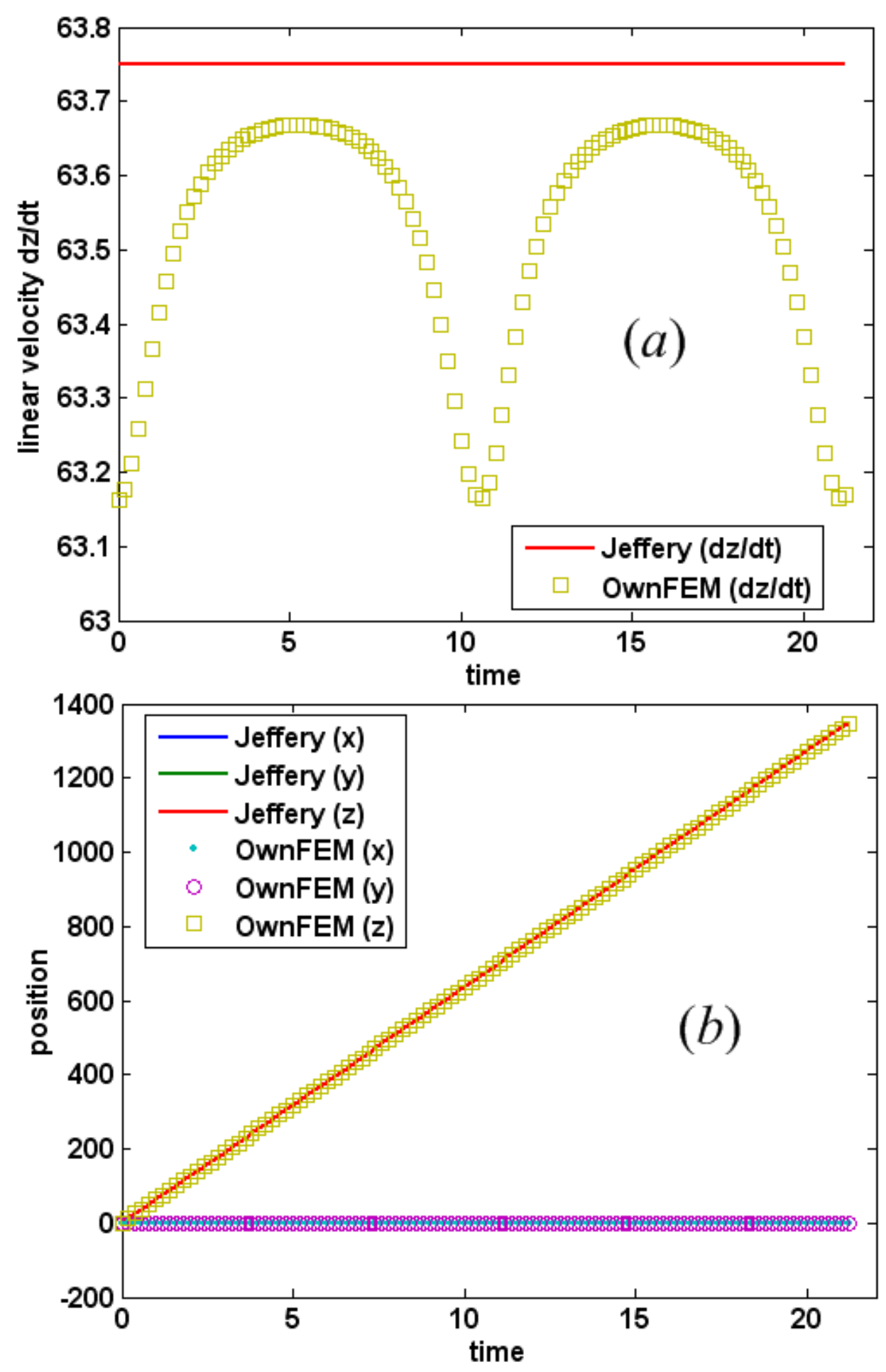

Figure 3.12: Fiber translation in a general plane Poiseuille flow: (a) fiber's linear velocity in the $z$ direction, and (b) fiber position. 
to Chwang's theoretic derivation. Meanwhile, in the Poiseuille flow, no lateral motion in the $y$ direction is detected, which confirms to Bretherton's theory.

\section{Circular Poiseuille flow}

For the circular Poiseuille flow, the velocity profile is a function of both $x$ and $y$ in the Cartesian coordinate system, as seen in Eq.(3.2). In this section, we use a large fluid domain instead of the circular tube to model the fiber motion, so as to eliminate the effect of the tube wall. The dimensionless circular Poiseuille flow profile with the suppressed $*$ is defined as $U_{x}=0, U_{y}=0, U_{z}=\frac{\nabla p}{4}\left[h^{2}-x^{2}-y^{2}\right]$, in which $\nabla p$ is the dimensionless pressure gradient and $h$ is the dimensionless radius of the tube. If fiber centroid does not lie on the centerline of the fluid, the flow profile can be decomposed into three flow components in the $\xi \eta \zeta$ coordinate system with fiber centroid at the centerline of fluid, defined as

uniform flow component:

$$
U_{\zeta}=\frac{\nabla p}{4}\left(h^{2}-x_{c}^{2}-y_{c}^{2}\right)
$$

shear flow component:

$$
U_{\zeta}=\frac{\nabla p}{4}\left(-2 x_{c} \xi-2 y_{c} \eta\right)
$$

and quadratic flow component:

$$
U_{\zeta}=\frac{\nabla p}{4}\left(-\xi^{2}-\eta^{2}\right)
$$

In a circular Poiseuille flow, fiber orientation is defined by the shear flow component, and fiber translation is controlled by the uniform and quadratic flow components. First, we investigate the effect of the sole quadratic flow component and compare it with that in the plane Poiseuille flow. In this example, the quadratic flow component in the $x y z$ coordinate system is defined as $U_{x}=U_{y}=0, U_{z}=\frac{\nabla p}{4}\left(-x^{2}-y^{2}\right)$, 

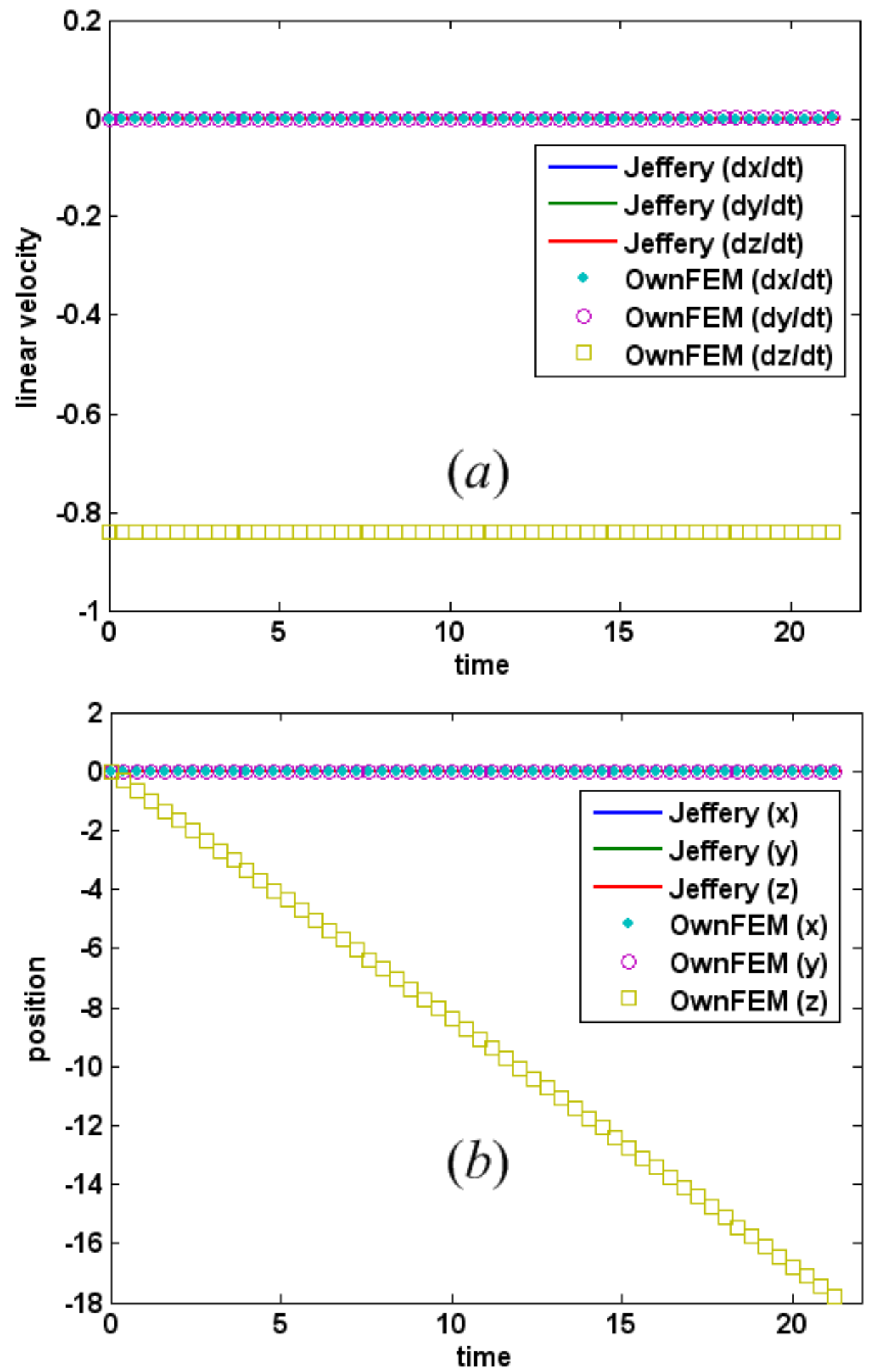

Figure 3.13: Change of fiber's linear velocity in a circular Poiseuille flow: $(a)$ fiber's linear velocity and (b) fiber position. 
where we set $\frac{\nabla p}{4}=1$ in order to eliminate the effect of coefficient $\nabla p$. The initial condition of fiber is $\phi=0, \theta=\pi / 2, \psi=0$ and $x_{c}=y_{c}=z_{c}=0$. The results of fiber motion is shown in Fig.3.13, from which we can see that fiber moves backwards, even though the quadratic flow component has zero velocity at fiber centroid. By comparing with the plane Poiseuille flow (cf.Fig.3.9), we can see that the change of fiber's linear velocity in the circular Poiseuille flow is larger than that in the plane Poiseuille flow. This can be explained by comparing the quadratic flow components of two Poiseuille flows. In the plane Poiseuille flow, there is only one quadratic term $-x^{2}$, while in the circular Poiseuille flow, there are two quadratic terms $-x^{2}$ and $-y^{2}$, which cause more effects on fiber's linear velocity.
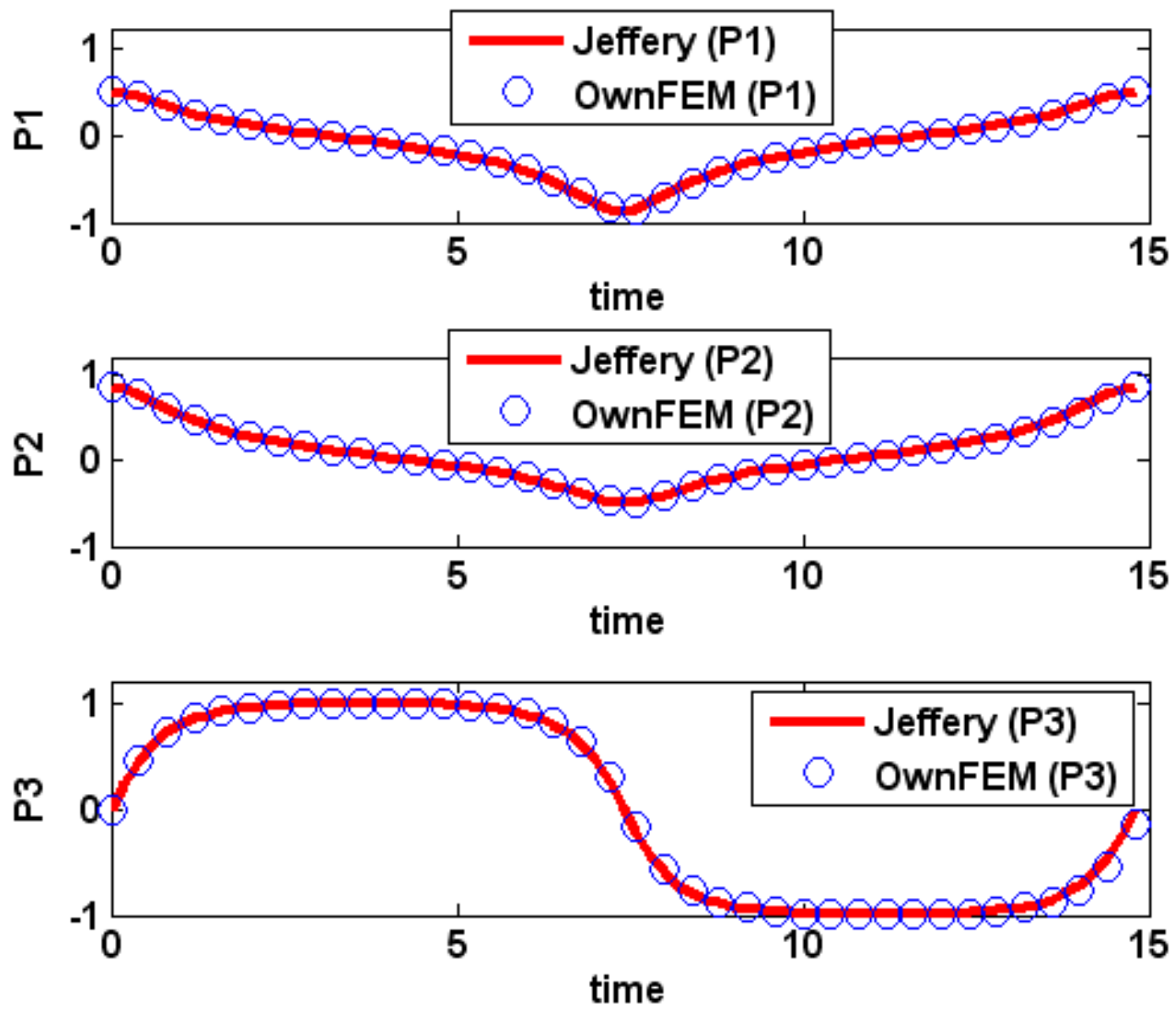

Figure 3.14: Fiber orientation in a circular Poiseuille flow

Next, we investigate fiber orientation and translation in a general circular Poiseuille 

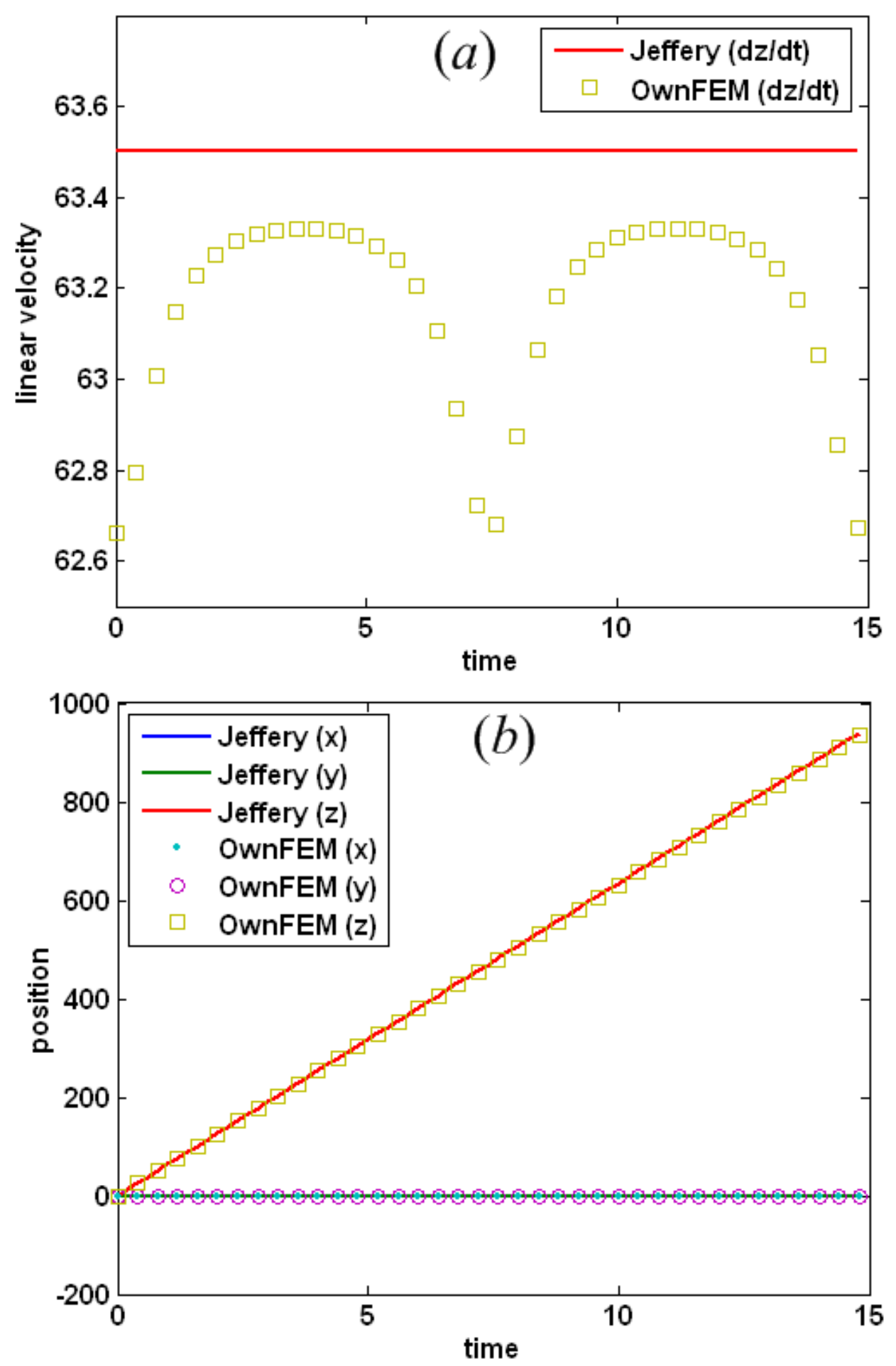

Figure 3.15: Fiber translation in a circular Poiseuille flow in a tube: (a) fiber's linear velocity in the $z$ direction and (b) fiber position. 
flow when fiber centroid does not lie along the centerline of the flow field. The dimensionless undisturbed flow field is defined as $U_{x}=U_{y}=0, U_{z}=\frac{\nabla p}{4}\left[h^{2}-x^{2}-y^{2}\right]$, where $h=8$ and $\frac{\nabla p}{4}=1$. The initial condition of fiber is set as $\phi=0, \theta=\pi / 2, \psi=0$ and $x_{c}=-0.5, y_{c}=-0.5, z_{c}=0$. In this case, the shear rate evaluated at fiber centroid is $\gamma=\gamma_{x}+\gamma_{y}$, where $\gamma_{x}=-\left.2 x\right|_{x=-0.5}=1$, and $\gamma_{y}=\left.2 y\right|_{y=-0.5}=1$. Fiber orientation in this circular Poiseuille flow is compared with that in the homogeneous flow $U_{z}=x+y$ using Eq.(1.6). The results of fiber orientation defined in the components of unit direction $\mathbf{P}$ are shown in Fig.3.14, from which we can see that fiber orientation is governed by Jeffery's equation with the equivalent shear rate evaluated at fiber centroid.

In terms of fiber translation, results show that fiber does not have the lateral motion in the $x$ or $y$ directions. The fiber follows the Poiseuille flow in the $z$ direction, but the change of linear velocity is observed in the numerical results, as seen in Fig.3.15.

\subsection{Summary}

This chapter presents a unique stand-alone three-dimensional FEM-based simulation tool which is coupled with the Newton-Raphson method and Runge-Kutta iterative method to model fiber motions in various unbounded homogeneous and inhomogeneous Newtonian flows. Fiber motions in plane and circular Poiseuille flows are investigated, and compared with existing theories and experimental findings. Our results confirm to Bretherton's conclusion that the Segré-Silberberg phenomenon does not exist in unbounded creeping Poiseuille flows. In a Poiseuille flow, the fiber performs a tumbling motion, which is governed by Jeffery's equation using the equivalent shear rate of the flow evaluated at fiber centroid. Another interesting finding is that the fiber has a change of linear velocity in the direction of the flow field. This is caused 
by the quadratic flow component, with the hydrodynamic force dragging the fiber backwards. As fiber rotates, the driving force changes accordingly. This phenomenon is not observed in Stover's experiments, because the change of linear velocity is small compared with the fluid velocity evaluated at fiber centroid. 


\section{Chapter 4}

\section{Brownian Dynamics Simulation of Nano-Fiber Suspensions for Nano-Fiber Composites Processing}

\subsection{Introduction}

Nano-tube/nano-fiber reinforced polymers have exceptional material properties [66, 67]. While Jeffery's model has received wide acceptance in the mold filling community, the motions of nano-fibers in nano-composites are too complicated to be predicted only by Jeffery's theory. The simulation of nano-fibers suspended in the polymeric melt must consider Brownian motions, which arise from the thermal fluctuations exerted from the surrounding fluid molecules. Brownian motions affect the rheological properties of polymeric composites [68] as explored by Hinch [69], who proposed a constitutive equation to account for the Brownian effects. Computational simulation has recently become an effective tool to numerically study Brownian suspension [7075]. The commonly used methods are Brownian Dynamics Simulation (BDS) [7073], Molecular Dynamic Simulation (MDS) [74] and the Monte Carlo method [75]. Of these, BDS method has been shown to provide an efficient means to model the 
Brownian motions of nano-fibers. Recent research has used rod-like [70], plate-like [71, 72], or bead-rod [73] models to approximate a nano-fiber. Unfortunately, these models require connecting forces between adjacent beads or rods, which over-complicates the calculations.

\subsection{FEM-based Brownian dynamics simulation}

Micro or sub-micro fibers within a viscous fluid perform two kinds of Brownian motions: translational and rotational motions. The effect of Brownian motion is quantified by the dimensionless number, Péclect number, which describes the ratio of shear rate to the rate of diffusion. We use $P e$ and $P e_{r}$ to represent the translational and rotational Péclect numbers respectively. For a sphere, the Péclect numbers are calcualted as

$$
P e=\frac{(0.5 a)^{2} \dot{\gamma}}{D} \quad \text { and } \quad P e_{r}=\frac{\dot{\gamma}}{D_{r}}
$$

where $D$ is the translational Brownian diffusivity, i.e. the rate at which fiber changes linear velocity caused by the translational Brownian effect. In the same manner, $D_{r}$ represents the rotational Brownian diffusivity, which results from the rotational Brownian effect. The diffusivity of a sphere is calculated as

$$
D=\frac{K_{B} T_{e}}{3 \pi \mu a} \quad \text { and } \quad D_{r}=\frac{K_{B} T_{e}}{\pi \mu a^{3}}
$$

where $k_{B}$ is the Boltzmann constant, and $T_{e}$ is the absolute temperature. It can be seen that both translational and rotational Péclect numbers are proportional to the third order of fiber size. At a low Péclect number $(\ll 1)$, Brownian motion has a great effect on fiber motion, while at a high Péclect number ( $\gg 1)$, Brownian effect is insignificant and hydrodynamic effect dominates the fiber motion. When the Péclect 
number is comparable to 1, both Brownian and hydrodynamic effects come into play.

In this chaper, a FEM-based Brownian dynamics simulation is applied to solve the Langevin equation, a stochastic differential equation, which accounts for the Brownian effect of nano-fibers and hydrodynamic effect from the bulk fluid. The Langevin equation with the neglected inertia of nano-fibers (as in Jeffery's theory) yields

$$
\begin{aligned}
\mathbf{F}^{H}+\mathbf{F}^{B} & =\mathbf{0} \\
\mathbf{T}^{H}+\mathbf{T}^{B} & =\mathbf{0}
\end{aligned}
$$

where $\mathbf{F}^{H}$ and $\mathbf{T}^{H}$ represent, respectively, the hydrodynamic force and torque exerted by the surrounding fluid, which are obtained using the Finite Element Method, presented in Chapter 3, and $\mathbf{F}^{B}$ and $\mathbf{T}^{B}$, respectively, represent the Brownian force and torque caused by the random molecular collisions. Note that $\mathbf{F}^{B}$ and $\mathbf{T}^{B}$ are both stochastic variables, which are modeled using Gaussian random functions. Fiber's velocities are obtained by solving Eqs.(4.3) and (4.4) simultaneously using a NewtonRaphson iteration method. Fiber orientation and position are obtained using a $4^{\text {th }}$ order Runge-Kutta method.

Random collisions from the surrounding fluid molecules are regarded as the random thermal disturbing forces and torques. In Eqs.(4.3) and (4.4), $\mathbf{F}^{B}$ and $\mathbf{T}^{B}$ follow Gaussian distributions defined by the corresponding first and second moments as

$$
\begin{array}{rlrl}
\left\langle\mathbf{F}^{B}(t)\right\rangle & =\mathbf{0}, & & \left\langle\mathbf{F}^{B}(t) \mathbf{F}^{B}(t+\Delta t)\right\rangle=2 \Re k_{B} T_{e} \delta(\Delta t) \\
\left\langle\mathbf{T}^{B}(t)\right\rangle=\mathbf{0}, & & \left\langle\mathbf{T}^{B}(t) \mathbf{T}^{B}(t+\Delta t)\right\rangle=2 \bar{\Re} k_{B} T_{e} \delta(\Delta t)
\end{array}
$$

where the angle bracelet indicates the ensemble average, $\Delta t$ is the time interval, $\delta(\Delta t)$ is the Dirac delta function. The parameters $\Re$ and $\bar{\Re}$ are the resistance friction 
vectors, related to fiber's geometry. For an ellipsoidal fiber, $\Re$ and $\bar{\Re}$ are defined as

$$
\Re=3 \pi \mu a\left[\begin{array}{c}
X^{A} \\
Y^{A} \\
Y^{A}
\end{array}\right] \quad \bar{\Re}=\pi \mu a^{3}\left[\begin{array}{c}
X^{C} \\
Y^{C} \\
Y^{C}
\end{array}\right]
$$

where $X^{A}, Y^{A}, X^{C}, Y^{C}$ are the resistance functions for an ellipsoidal fiber [76]. Among the resistance functions, $X^{A}$ and $X^{C}$ represent the resistance along fiber's long axis, while $Y^{A}$ and $Y^{C}$ represent the resistance along fiber's short axis. The resistance functions are defined as

$$
\begin{aligned}
X^{A} & =\frac{8}{3} e^{3}\left[-2 e+\left(1+e^{2}\right) L\right]^{-1} \\
Y^{A} & =\frac{16}{3} e^{3}\left[2 e+\left(3 e^{2}-1\right) L\right]^{-1} \\
X^{C} & =\frac{4}{3} e^{3}\left(1-e^{2}\right)\left[2 e-\left(1-e^{2}\right) L\right]^{-1} \\
X^{C} & =\frac{4}{3} e^{3}\left(2-e^{2}\right)\left[-2 e+\left(1+e^{2}\right) L\right]^{-1}
\end{aligned}
$$

where $L=\ln \left(\frac{1+e}{1-e}\right)$ and $e=\sqrt{1-\frac{1}{r_{e}^{2}}}$. Therefore, the Brownian force and torque in the $x^{\prime} y^{\prime} z^{\prime}$ system are

$$
\begin{aligned}
\mathbf{F}^{B} & =\frac{1}{\Delta t} \sqrt{2 \Re k_{B} T} d \mathbf{W} \\
\mathbf{T}^{B} & =\frac{1}{\Delta t} \sqrt{2 \overline{\Re k_{B} T}} d \mathbf{W}
\end{aligned}
$$

where $d \mathbf{W}$ is the Wiener process, i.e. the Gaussian distribution with the mean value to be zero and the standard deviation to be $\sqrt{d t}$. From Sec.3.3, the hydrodynamic force and torque are obtained by solving the non-dimensionalized governing equations, so the hydrodynamic force and torque are dimensionless, while the random Brownian 
force and torque still have units. Therefore, we non-dimensionlize the Brownian force and torque using the same characteristic length $L_{c}$ and velocity $U_{c}$ as those in Sec.3.3, shown as

$$
\begin{aligned}
\mathbf{F}^{B} & =\frac{\frac{1}{\Delta t} \sqrt{2 \Re k_{B} T} d \mathbf{W}}{\mu U_{c} L_{c}} \\
\mathbf{T}^{B} & =\frac{\frac{1}{\Delta t} \sqrt{2 \bar{\Re} k_{B} T} d \mathbf{W}}{\mu U_{c} L_{c}^{2}}
\end{aligned}
$$

In the Langevin equation, the hydrodynamic effect is a function of fiber's velocities, while the Brownian effect is obtained using the Gaussian random number generator. Then fiber's velocities can be obtained by solving the Langevin equation, as seen in Eqs.(4.3) and (4.4).

The Newton-Raphson iteration algorithm is used to compute fiber's linear and angular velocities at any time instant $t_{i}$. The Langevin equation with both dimensionless hydrodynamic and Brownian effects is summarized as

$$
\begin{gathered}
\mathbf{F}^{H}\left(\dot{x}_{c i}, \dot{y}_{c i}, \dot{z}_{c i}, \dot{\phi}_{i}, \dot{\theta}_{i}, \dot{\psi}_{i}\right)+\frac{\frac{1}{\Delta t} \sqrt{2 \Re k_{B} T} d \mathbf{W}}{\mu U_{c} L_{c}}=\mathbf{0} \\
\mathbf{T}^{H}\left(\dot{x}_{c i}, \dot{y}_{c i}, \dot{z}_{c i}, \dot{\phi}_{i}, \dot{\theta}_{i}, \dot{\psi}_{i}\right)+\frac{\frac{1}{\Delta t} \sqrt{2 \bar{\Re} k_{B} T} d \mathbf{W}}{\mu U_{c} L_{c}^{2}}=\mathbf{0}
\end{gathered}
$$

Fiber's linear and angular velocities at any time instant $t_{i}$ are obtained by solving Eqs.(4.12) and (4.13) simultaneously as $\dot{\mathbf{y}}_{I+1}=\dot{\mathbf{y}}_{I}-\left[\mathbf{J}_{I}\right]^{-1}\left[\mathbf{Q}_{I}\right]$, where $I$ is the NewtonRaphson iteration number, $\dot{\mathbf{y}}_{I}=\left[\dot{x}_{c i}, \dot{y}_{c i}, \dot{z}_{c i}, \dot{\phi}_{i}, \dot{\theta}_{i}, \dot{\psi}_{i}\right]^{T}$, and $\left[\mathbf{Q}_{I}\right]=\left[\mathbf{F}^{H}+\mathbf{F}^{B} ; \mathbf{T}^{H}+\right.$ $\left.\mathbf{T}^{B}\right]$, which is a function of the reaction force $\mathbf{F}_{e}$ in the finite element model. The Jacobian matrix $\left[\mathbf{J}_{I}\right]$ is derived analytically as

$$
\frac{\partial \mathbf{Q}_{I}}{\partial \dot{\mathbf{y}}_{I}}=\left[\frac{\partial \mathbf{F}^{H}}{\partial \dot{\mathbf{y}}_{I}}+\frac{\partial \mathbf{F}^{B}}{\partial \dot{\mathbf{y}}_{I}} ; \frac{\partial \mathbf{T}^{H}}{\partial \dot{\mathbf{y}}_{I}}+\frac{\partial \mathbf{T}^{B}}{\partial \dot{\mathbf{y}}_{I}}\right]=\left[-\sum_{n=1}^{N} \frac{\partial\left(\mathbf{F}_{\mathbf{e}}\right)_{n}}{\partial \dot{\mathbf{y}}_{I}} ;-\sum_{n=1}^{N}\left(\mathbf{r}_{e}\right)_{n} \times \frac{\partial\left(\mathbf{F}_{\mathbf{e}}\right)_{n}}{\partial \dot{\mathbf{y}}_{I}}\right]
$$


in which $\frac{\partial \mathbf{F}_{\mathbf{e}}}{\partial \dot{\mathbf{y}}_{I}}$ is calculated in Eq.(3.21) analytically. Therefore, at any time instant $t_{i}$, with the given fiber position $x_{c i}, y_{c i}, z_{c i}$ and fiber orientation $\phi_{i}, \theta_{i}, \psi_{i}$, the linear velocity $\left(\dot{x}_{c i}, \dot{y}_{c i}, \dot{z}_{c i}\right)$ and angular velocity (defined by $\left.\dot{\phi}_{i}, \dot{\theta}_{i}, \dot{\psi}_{i}\right)$ are then obtained from the converged Newton-Raphson iteration to give $\dot{\mathbf{y}}_{i}=\mathbf{f}\left(t_{i}, \mathbf{y}_{i}\right)$, where $\mathbf{y}_{i}=\left[x_{c i}, y_{c i}, z_{c i}, \phi_{i}, \theta_{i}, \psi_{i}\right]^{T}$. A $4^{t h}$-order Runge-Kutta algorithm is used to track the evolution of $x_{c}, y_{c}, z_{c}, \phi, \theta, \psi$ as a function of time, as seen in Chapter 3.

For the consideration of random Brownian motions, Monte-Carlo simulation is used to evaluate the motions of a great many fibers associated with different random Brownian forces and torques. The final fiber motion is obtained by averaging numerous fiber motions. Brownian force and torque are generated using the Gaussian random functions. Therefore, Eqs.(4.12) and (4.13) are a pair of differential equations with a stochastic term and Finite Element Analysis. In this chapter, the motion of a single fiber is considered. At any time instant $t_{i}$, Brownian force and torque are generated using Eqs.(4.10) and (4.11), and used to solve fiber's velocities. Then fiber orientation and position will be obtained. In order to gain a statistical view of fiber motion, we used the Monte-Carlo simulation to obtain a large number of fiber's motion paths, associated with a large number of Brownian forces and torques. When all motion paths are obtained, the mean and standard deviation of fiber paths can be calculated using the ensemble average of all motion paths, defined as

$$
\begin{aligned}
\overline{\mathbf{y}} & =\langle\mathbf{y}\rangle=\frac{1}{L} \sum_{l=1}^{L} \mathbf{y}_{l} \\
\sigma_{\mathbf{y}} & =\sqrt{\frac{1}{L-1} \sum_{l=1}^{L}\left(\mathbf{y}_{l}-\overline{\mathbf{y}}\right)^{2}}
\end{aligned}
$$

where $l$ is the index of different fiber's motion paths associated with different Brownian force and torque and $L$ is the total number of samples in the Monte-Carlo simulation. 


\subsection{Implementation and examples}

In this research work, a three-dimensional finite element simulation tool has been proposed, but three-dimensional Monte-Carlo simulation is hardly to achieved due to the tremendous computation time for a large number of samples. Therefore, in this chapter, two-dimensional Monte-Carlo simulation is implemented, and we demonstrate that two-dimensional fiber motion is the same as three-dimensional planar motion, as seen in Sec.4.3.1.

\subsubsection{Comparison of two- and three-dimensional fiber mo- tions}

In this section, we repeat the three-dimensional simulation using the following initial condition: $\theta_{0}=\pi / 2, \phi_{0}=\psi_{0}=0$ and $y_{c 0}=1, x_{c 0}=z_{c 0}=0$. Therefore, the fiber exists in the $y z$ plane initially, and the angles $\theta$ and $\psi$ remain unchanged in the three-dimensional FEM.

For the two-dimensional FEM, we use the triangular meshes to represent the fluid domain in the $y z$ plane. Fiber orientation is only determined by the angle $\phi$, while fiber position is determined by $y_{c}$ and $z_{c}$. Initially, $\phi=0, y_{c}=1$ and $z_{c}=0$. The undisturbed simple shear flow is defined as $U_{z}=\dot{\gamma} y$, where $\dot{\gamma}=1$. The plots of $\phi$ and $y_{c}, z_{c}$ in the three-dimensional finite element model are compared with those in the two-dimensional model, as shown in Fig.4.1. The average true percent relative error of angle $\phi$ calculated in planar three-dimensional FEM and two-dimensional FEM are both below $0.6 \%$. Therefore, for the planar motion of fiber, two-dimensional FEM can be used to study the fiber motion, and the results are in excellent agreement with Jeffery's theory. The two-dimensional FEM simulation only takes 30 seconds for one complete period of fiber orientation, which makes possible the Monte-Carlo simulation with a large sample size. 

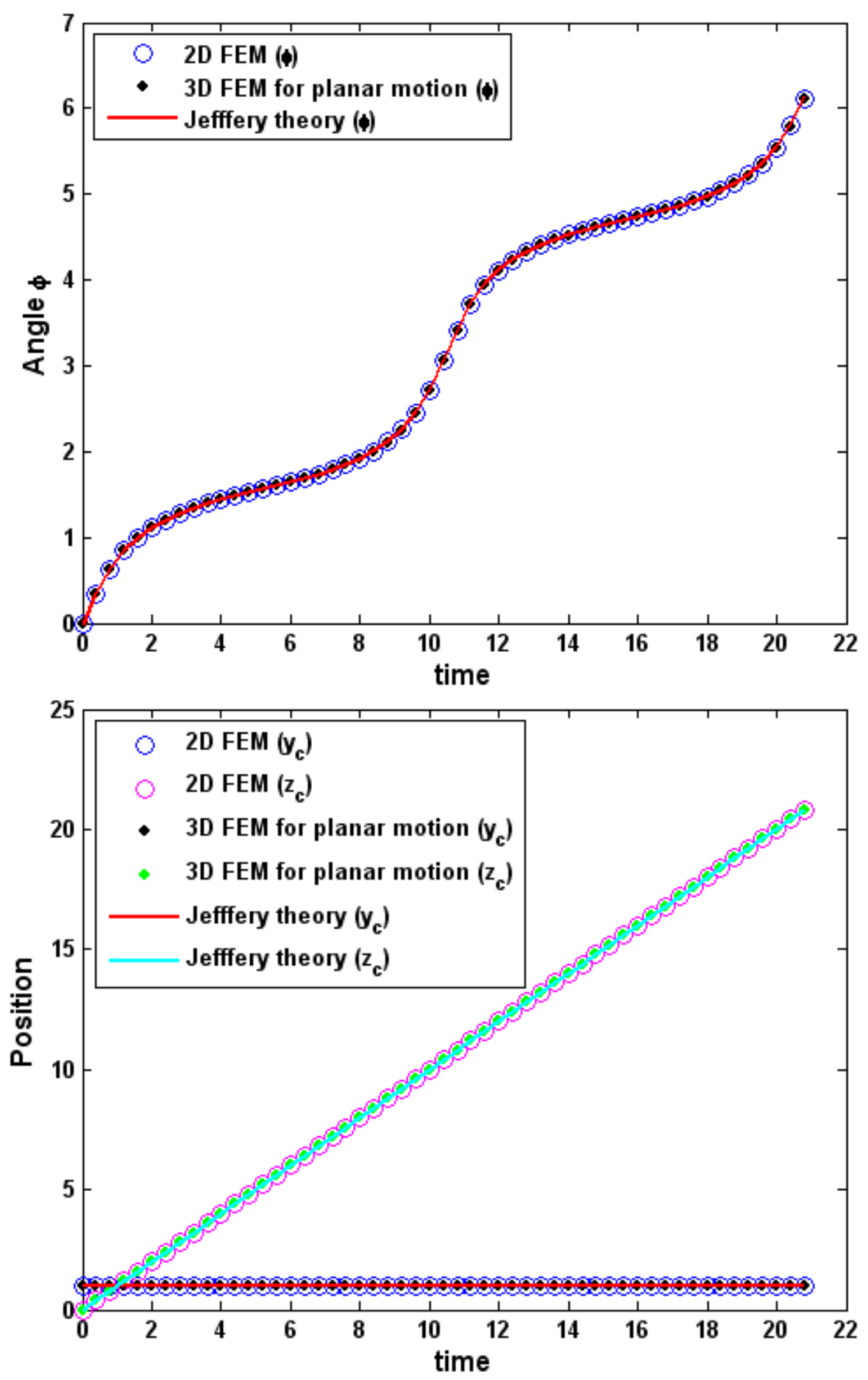

Figure 4.1: Comparison of three-dimensional FEM in solving fiber's planar motion and two-dimensional FEM: (a) Fiber orientation $\phi ;(b)$ Fiber position $z_{c}$ and $y_{c}$.

In the proposed finite element model, we have access to the fluid velocity and pressure at all nodes of the fluid domain. Therefore, we investigate the velocity, 
pressure, and stress disturbance caused by the fiber motion at $t_{i}=0.8$, as seen in Fig.4.2, from which we can see that fiber motion has a great effect on the bulk fluid in the region around fiber surface. For instance, the shear rate of the undisturbed simple shear flow is $\dot{\gamma}=1$ for this case. But if we look at the distribution of shear rate around fiber surface in Fig.4.2(d), the shear rate close to fiber surface is about 4, while in the region with a finite distance from fiber surface, the shear rate becomes to be 1 . Therefore, the fiber motion has a local disturbance on the fluid field. In the following sections, we further look at how the bulk fluid is disturbed by the Brownian motions of sub-micro fibers.

In the following sections, three examples using two-dimensional FEM-based MonteCarlo simulation are presented to explore the Brownian motions of fibers with various Péclet numbers in a simple shear flow $\left(U_{y}=0, U_{z}=\dot{\gamma} y\right)$. Since the Brownian force and torque also depend on fiber's shape (described by geometric aspect ratio), we study the Brownian effects using ellipsoidal fibers with various geometric aspect ratios.

\subsubsection{Brownian motions of a sphere with $r_{e}=1$}

The first example starts with a sphere $(a=b)$. In this example, we investigate the motion of fibers with different fibre sizes $(a$ or $b): 20 \mathrm{~nm}, 200 \mathrm{~nm}$, and $2000 \mathrm{~nm}$. The parameters used in this example are tabulated in Table 4.1, from which we can see that the nano-size fiber results in a small Péclet number, while large size of the fiber causes a large Péclet number. In this section, we investigate how the Brownian motion affects the fiber suspension.

The dimensional initial fiber position and orientation (two-dimensional case) for those three cases are $\phi_{0}=0$ and $y_{c 0}=1, z_{c 0}=0$. The number of samples used in the Monte-Carlo simulation is 10000. The results of fiber orientation $(\phi)$ and angular

velocity $(\dot{\phi})$ for those three cases are tabulated in Table 4.2 , from which it can be 


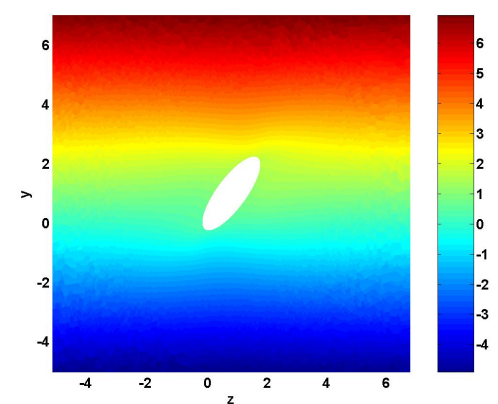

(a) z velocity $(-4.5 \sim 6.5)$

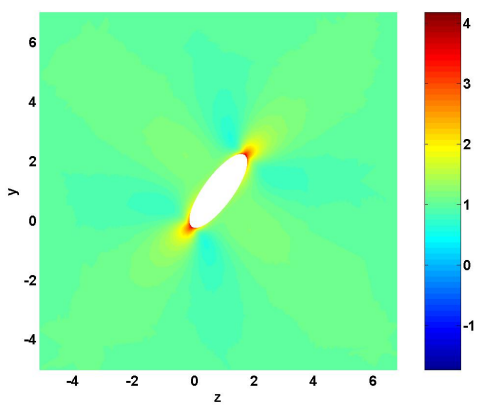

(d) Shear rate $\dot{\gamma}(0.63 \sim 4.22)$

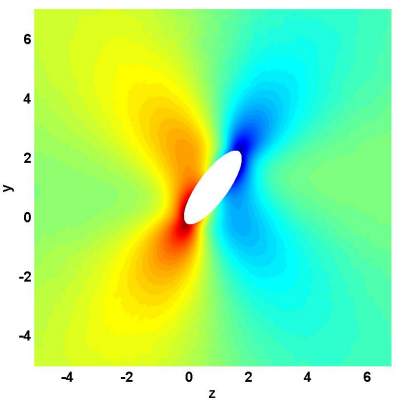

(b) y velocity $(-0.6 \sim 0.6)$

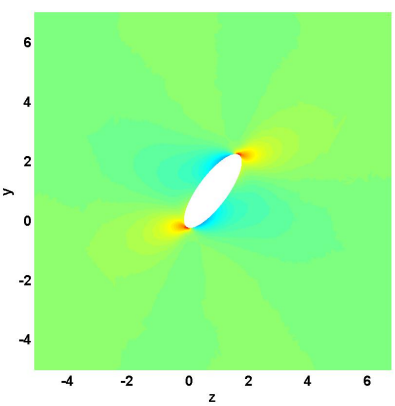

(e) $\sigma_{z z}(-2.2 \sim 4.9)$
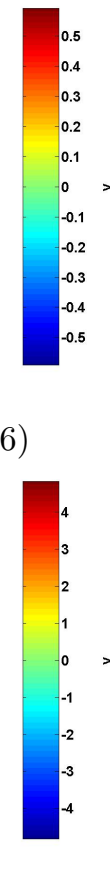

(c) Pressure $(-3.8 \sim 1.3)$
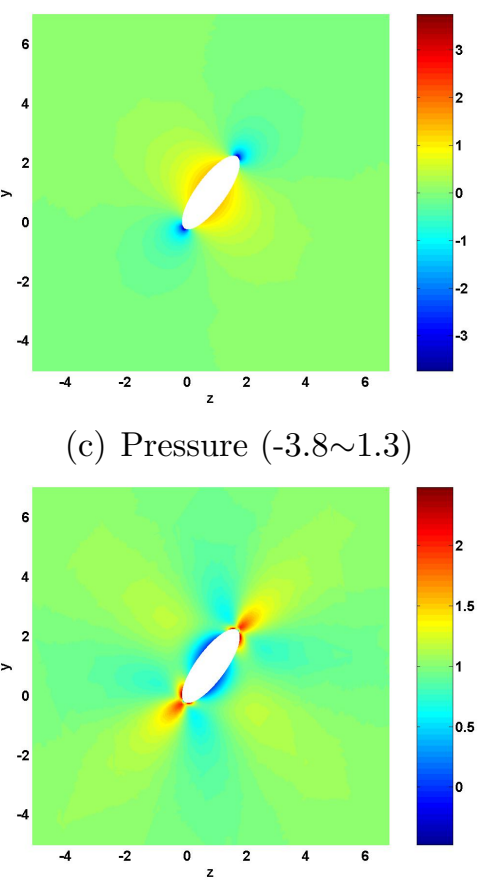

(f) $\sigma_{y z}$ or $\sigma_{z y}(-0.008 \sim 2.5)$

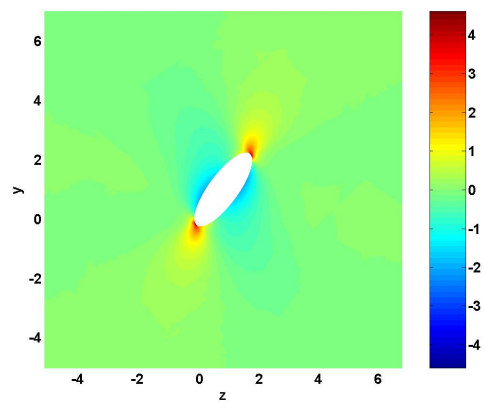

(g) $\sigma_{y y}(-2.02 \sim 4.67)$

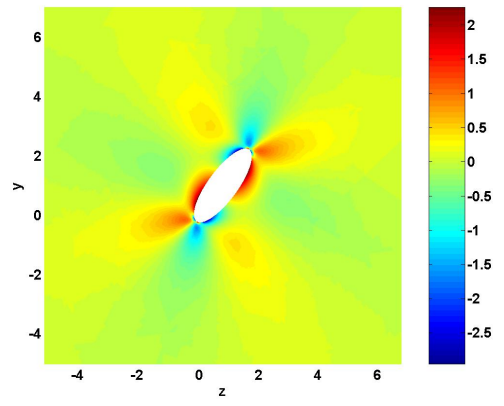

(h) $\sigma_{z z}-\sigma_{y y}(-3 \sim 2.29)$

Figure 4.2: Disturbance of fiber motion on fluid velocity, pressure and stress around fiber surface at $t_{i}=0.8$ without Brownian motion

\begin{tabular}{|c|c|c|c|}
\hline & Case 1 & Case 2 & Case 3 \\
\hline \hline Fiber sizes & $a=b=20 \mathrm{~nm}$ & $a=b=200 \mathrm{~nm}$ & $a=b=2000 \mathrm{~nm}$ \\
\hline Péclet numbers & $P e=0.002, P e_{r}=0.003$ & $P e=2, P e_{r}=3$ & $P e=2000, P e_{r}=3000$ \\
\hline Characteristic length & $L_{c}=10 \mathrm{~nm}$ & $L_{c}=100 \mathrm{~nm}$ & $L_{c}=1000 \mathrm{~nm}$ \\
\hline Characteristic velocity & $U_{c}=10 \mathrm{~nm} / \mathrm{s}$ & $U_{c}=100 \mathrm{~nm} / \mathrm{s}$ & $U_{c}=1000 \mathrm{~nm} / \mathrm{s}$ \\
\hline Fluid properties & \multicolumn{2}{|c|}{ Viscosity: $\mu=1 \mathrm{~Pa} \cdot \mathrm{s}$, Shear rate: $\dot{\gamma}=1 \mathrm{~s}^{-1}$} \\
\hline Other properties & \multicolumn{2}{|c|}{$T=600 \mathrm{~K}, k_{B}=1.380648813 \times 10^{-23} \mathrm{~J} / \mathrm{K}$} \\
\hline Dimensionless properties & \multicolumn{2}{|c|}{ Fiber size: $a=b=2$, Shear rate: $\dot{\gamma}=1$} \\
\hline
\end{tabular}

Table 4.1: Parameters to simulate the motions of micro- and nano-fibers

seen that at a high Péclet number $\left(P e_{r}=3000\right)$, hydrodynamic effect dominates fiber motion, which is still governed by Jeffery's theory. The evolution of fiber orientation 
$(\phi)$ and angular velocity $(\dot{\phi})$ are shown in the third column of Table 4.2. At a medium Péclet number $\left(P e_{r}=3\right)$, both hydrodynamic and Brownian effects take effect. The periodic tumbling motion of a single fiber is disturbed by the random fluid molecular collisions, with the results shown in the second column of Table 4.2. At a low Péclet number $\left(P e_{r}=0.003\right)$, random Brownian effect takes control of fiber motions with the results shown in the first column of Table 4.2.

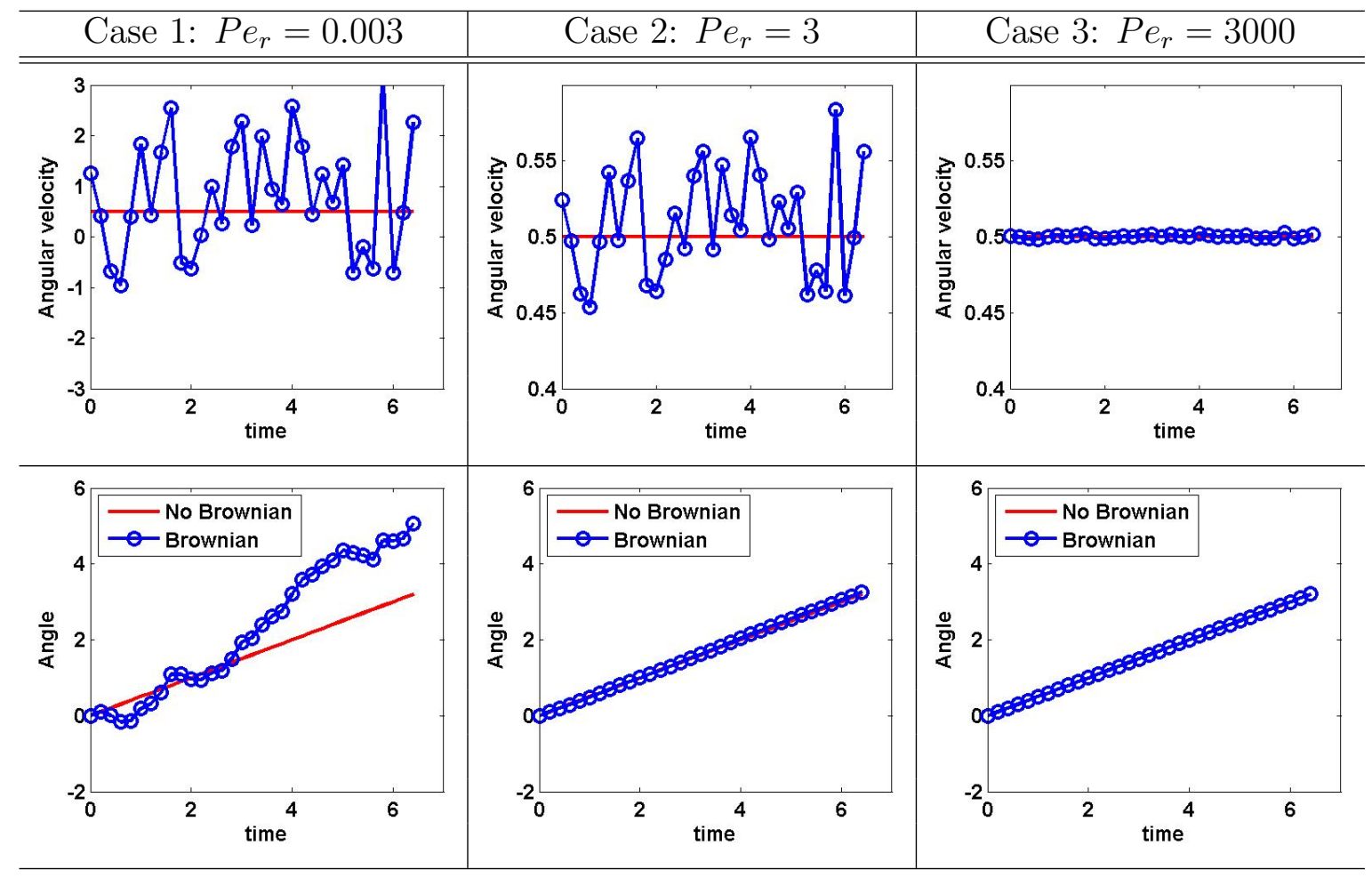

Table 4.2: Fiber orientations with different Péclet numbers $\left(r_{e}=1\right)$

In the Monte-Carlo simulatoin, 10000 fiber orientations are obtained using the random Brownian torques generated using a Gaussian distribution, and the final fiber orientation is calculated by averaging those 10000 paths in the Monte-Carlo simulation. The 10000 fiber paths is plotted at $t_{i}=5$ with the large and small Péclet numbers, which are shown in Fig.4.3. We can see that Brownian motion has a large effect on fiber orientation, with the standard deviation of $\phi$ to be around 115, as seen in Fig.4.3(a), while there is a small randomness of fiber orientation for a large Péclet number, with a very small standard deviation of $\phi$, as seen in Fig.4.3(b). 

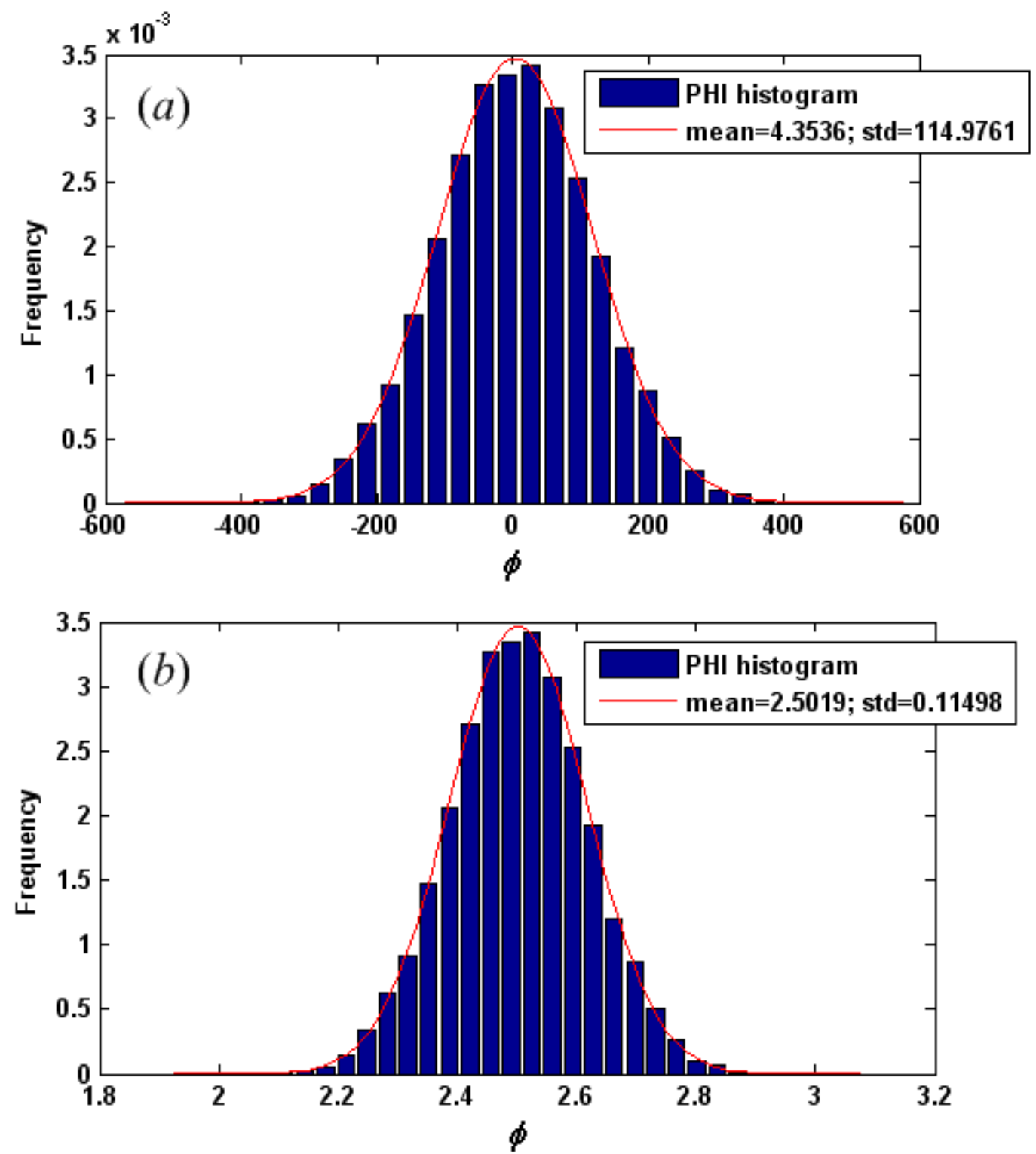

Figure 4.3: Distribution of fiber orientation at $t_{i}=5$ for small and large Péclet numbers: (a) $P e_{r}=0.003$ and $(b) P e_{r}=3000$.

The same phenomenon is observed in fiber's linear motion, as seen in Table 4.3. For a micro-fiber with a large $P e$ number, fiber motion is driven by the hydrodynamic force, while for a nano-fiber with a small $P$ e number, fiber motion is largely disturbed by the random Brownian forces from the fluid molecules. 


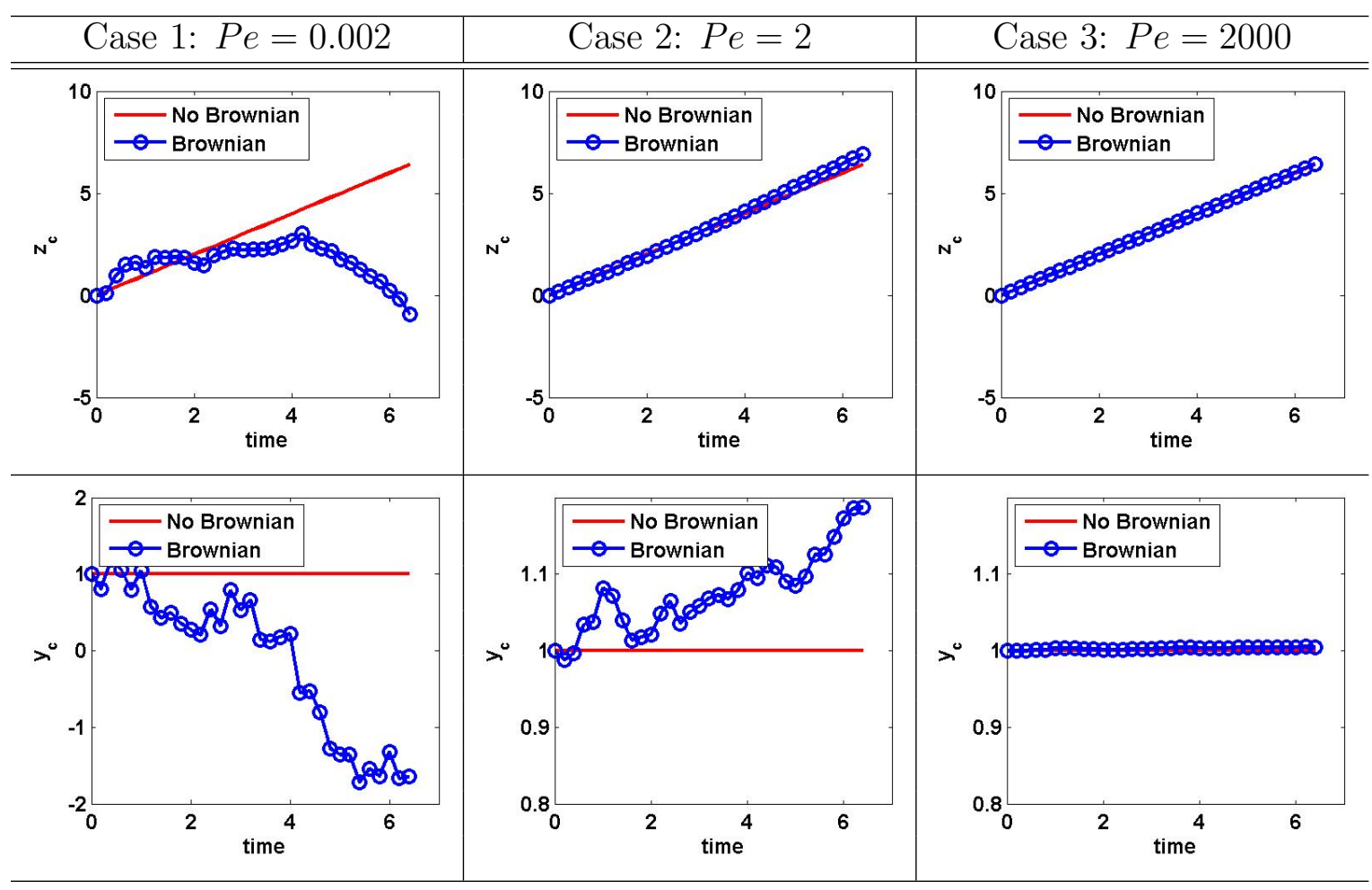

Table 4.3: Fiber positions with different Péclet numbers $\left(r_{e}=1\right)$

\subsubsection{Brownian motion of an ellipse with $r_{e}=3$}

In this example, we investigate three ellipsoidal fibers with different fiber sizes, which are tabulated in Table 4.4, in which the Péclet numbers are approximated by plugging the short axis of fiber into Eq.(4.1).

\begin{tabular}{c|c|c|c}
\hline & Case 1 & Case 2 & Case 3 \\
\hline \hline Fiber & $a=30 \mathrm{~nm}$ & $a=300 \mathrm{~nm}$ & $a=3000 \mathrm{~nm}$ \\
sizes & $b=10 \mathrm{~nm}$ & $b=100 \mathrm{~nm}$ & $b=1000 \mathrm{~nm}$ \\
\hline Péclet & $P e=0.0003$ & $P e=0.3$ & $P e=300$ \\
numbers & $P e_{r}=0.0004$ & $P e_{r}=0.4$ & $P e_{r}=400$ \\
\hline
\end{tabular}

Table 4.4: Fiber sizes and Péclet numbers used in the simulation

The geometric aspect ratios of three ellipses are 3. The same simple shear flow and initial fiber orientation and position are used as those in Sec.4.3.2. Fiber orientations with different Péclet numbers are tabulated in Table 4.5, which shows that Brownian 
effect is largely dependent on the fiber size, which is quantified by the Péclet number. As fiber decreases the size, Brownian effect arises.

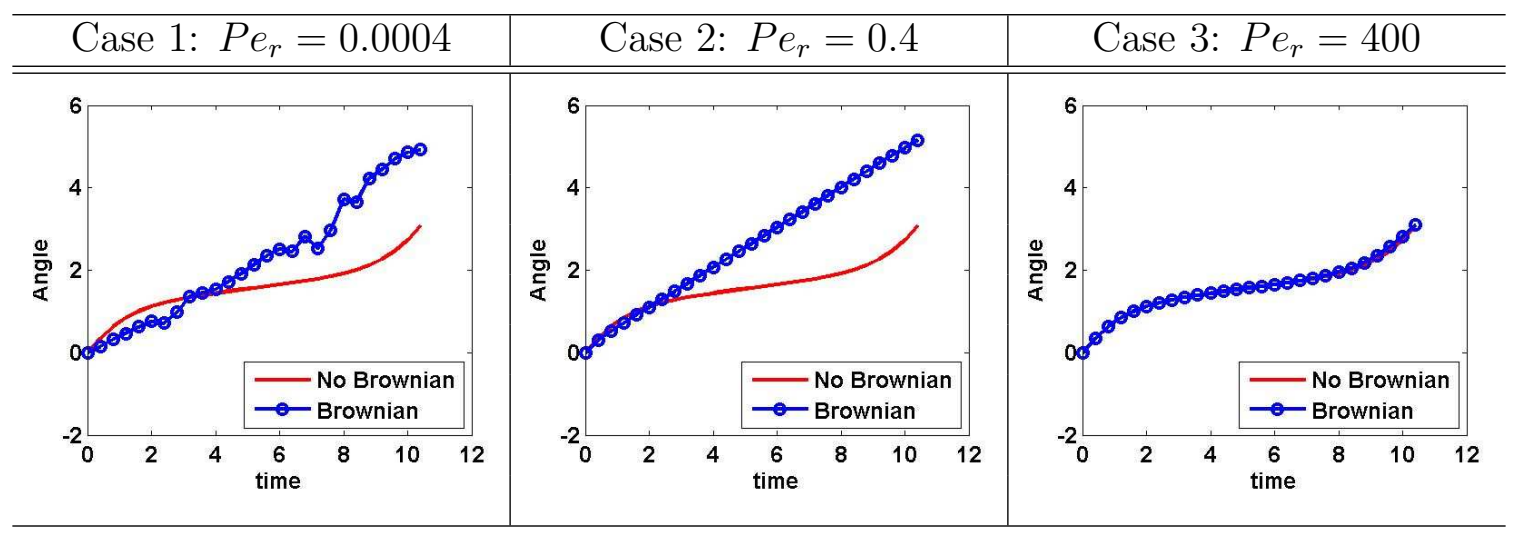

Table 4.5: Fiber orientations with different Péclet numbers $\left(r_{e}=3\right)$

The same phenomena for fiber's linear motion can be seen in Table 4.6, from which it can be seen that fiber movement is tremendously disturbed by molecular collisions with a small Péclet number, while fiber motion still follows undisturbed flow with a

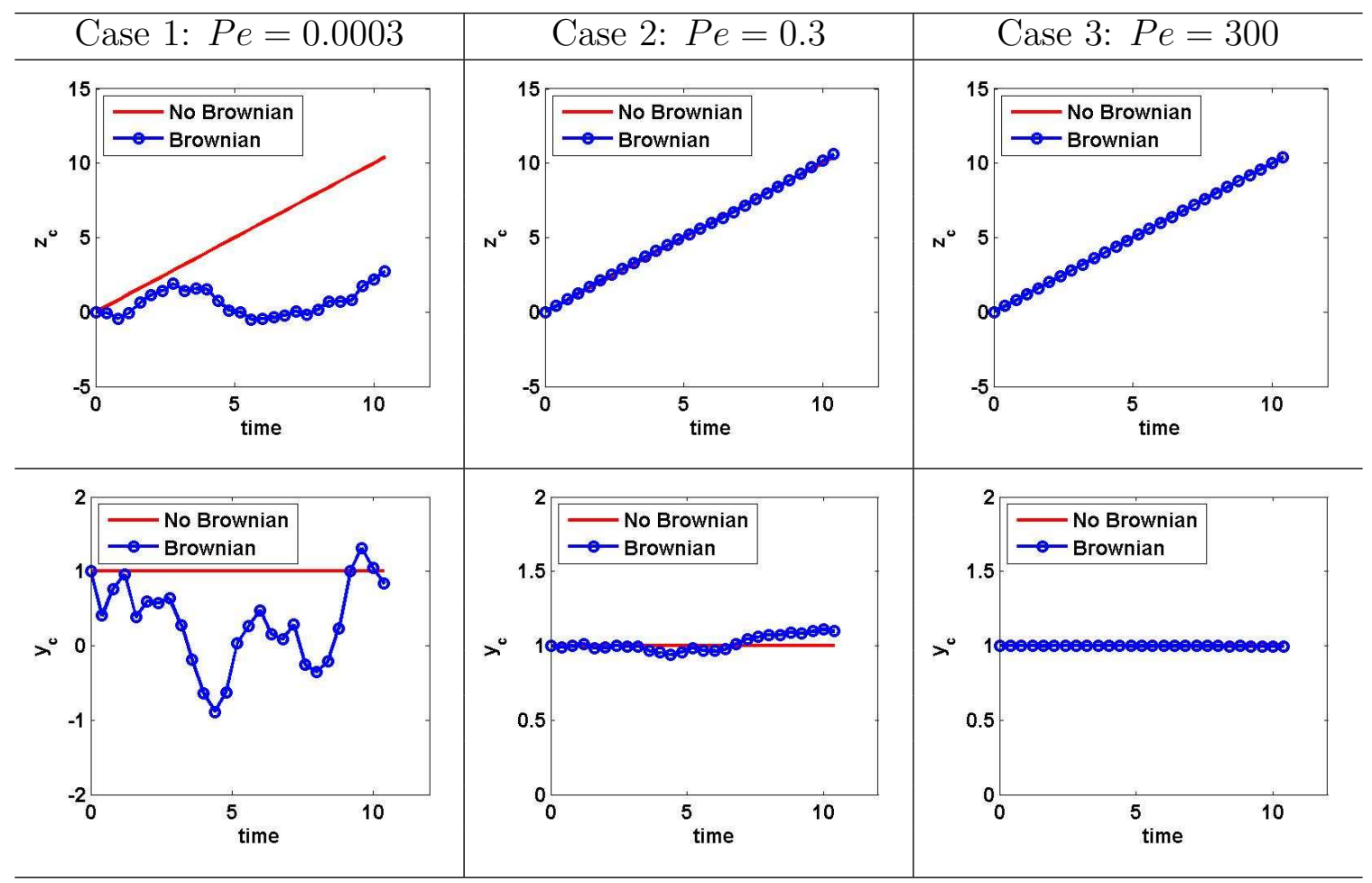

Table 4.6: Fiber positions with different Péclet numbers $\left(r_{e}=3\right)$ 
large Péclet number. At a medium Péclet number, both Brownian and hydrodynamic effects are active. Therefore, the consideration of Brownian effect with a small Péclet number is essential in nano-polymer composites processing.

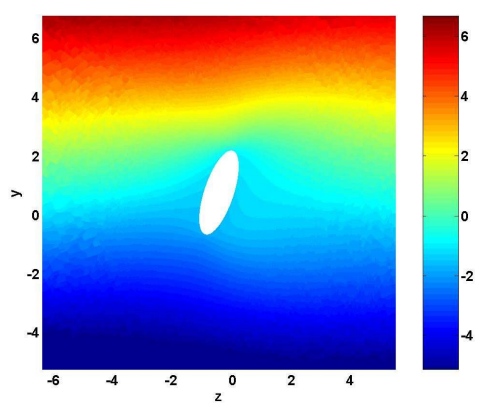

(a) z velocity $(-4.5 \sim 6.5)$

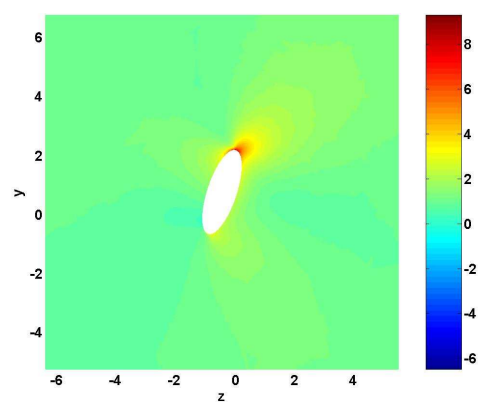

(d) Shear rate $(0.07 \sim 9.4)$

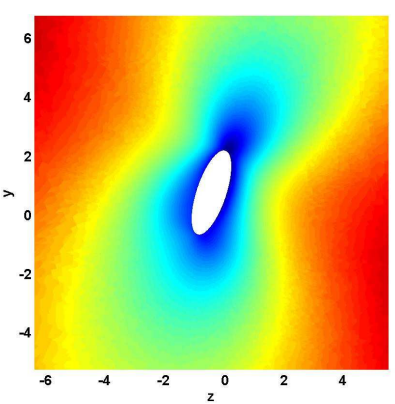

(b) y velocity $(-2.88 \sim-0.196)$

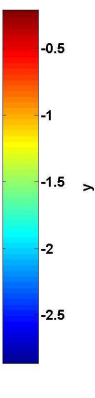

(e) $\sigma_{z z}(-6.5 \sim 10)$

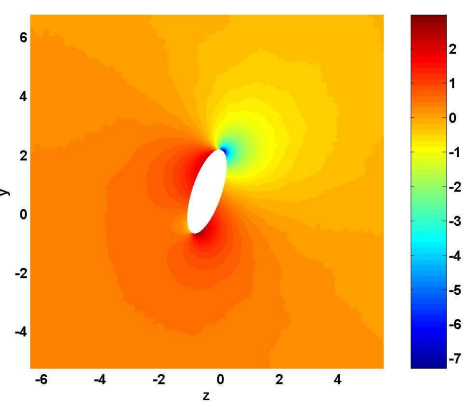

(c) Pressure (-7.37 3.05)
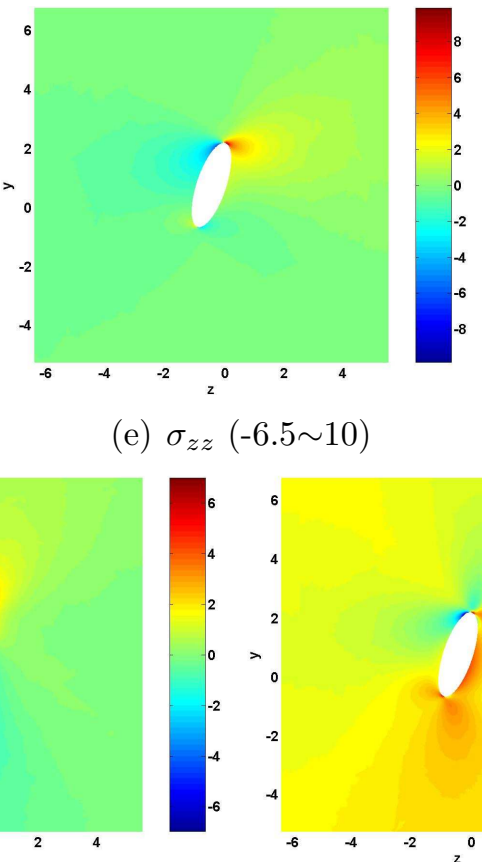

(g) $\sigma_{y y}(-3.5 \sim 7.1)$

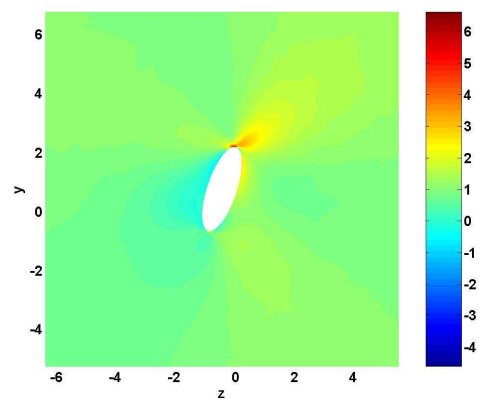

(f) $\sigma_{y z}$ or $\sigma_{z y}(-0.74 \sim 6.7)$

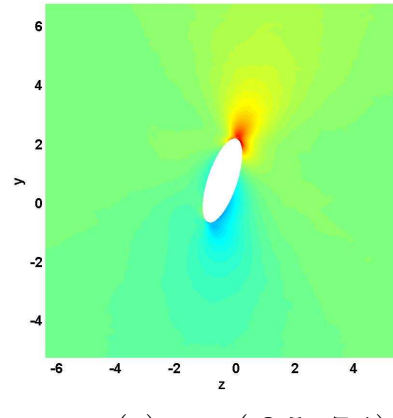

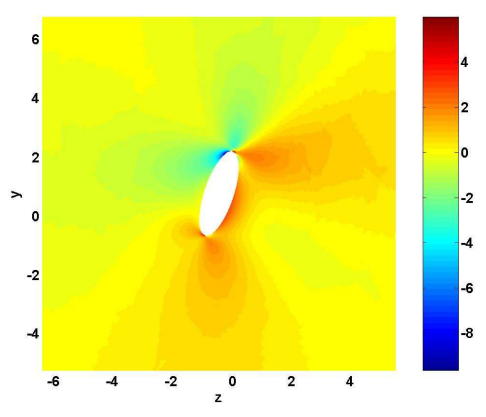

(h) $\sigma_{z z}-\sigma_{y y}(-9.78 \sim 6.1)$

Figure 4.4: Disturbance of fiber motion on fluid velocity, pressure and normal stress difference around fiber surface at $t_{i}=0.8$ with the Brownian motion

As discussed in Sec.4.2, fiber motion changes the local fluid velocity and pressure distribution, and the stress distribution around fiber surface. With the Brownian effect, fiber has the random motion, which further changes the fluid stress around fiber surface. In this section, we investigate the fluid velocity, pressure and stress in the region close to fiber surface at $t_{i}=0.8$ for Brownian fiber suspension, with the 
results shown in Fig.4.4, from which we can see that fiber's Brownian motion causes more disturbance on the fluid domain close to fiber surface. Take the normal stress difference as an example. The normal stress difference in Brownian motion is much larger than that in non-Brownian motion.

\subsubsection{Brownian motion of an ellipse with $r_{e}=10$}

Another example with a larger geometric aspect ratio is presented in this section. In this section, the fiber sizes are: $a=200 \mathrm{~nm}, b=20 \mathrm{~nm}$ and $a=20000 \mathrm{~nm}, b=$ $2000 \mathrm{~nm}$, and the geometric aspect ratios are both 10. The Péclet numbers are calculated using the short axis of fiber. So the Péclet numbers for both cases are respectively $P e=0.0003, P e_{r}=0.0004$ and $P e=300, P e_{r}=400$. Fiber motion with small and large Péclet numbers are tabulated in Table 4.7, from which we can see that Brownian effect causes the random motion of a nano-fiber.

\subsection{Summary}

In this chapter, we proposed a FEM-based Brownian dynamics simulation tool for modeling the motion of nano-fibers during the nano-composites processing. A standalone Finite Element Method is developed to study the hydrodynamic effect on the fiber and Brownian dynamics simulation is used to account for the Brownian effect. From our simulation results, we find that when fiber size decreases, Brownian effect comes into play and disturbs the fiber motion. The dimensionless Péclet number is calculated to quantify the Brownian effect. At a high Péclet number, fiber motion is still governed by Jeffery's theory, since the Brownian effect is insignificant. At a medium Péclet number, both hydrodynamic and Brownian effects come into play, and govern the fiber motion. At a small Péclet number, Brownian effect becomes very strong compared with the hydrodynamic effect, so fiber motion is tremendously 


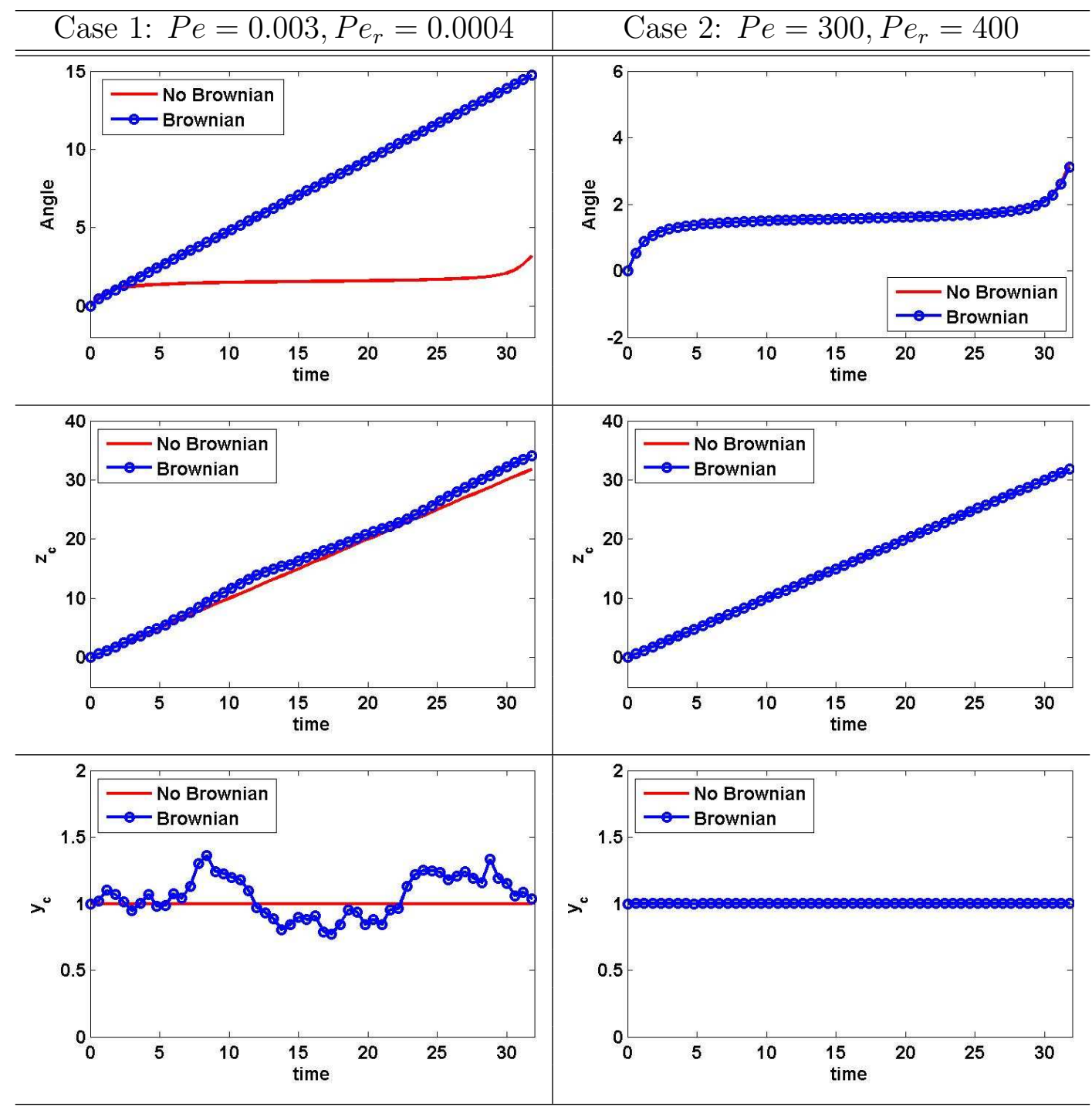

Table 4.7: Fiber motions with different Péclet numbers $\left(r_{e}=10\right)$

disturbed by the fluid molecules. In this chapter, two-dimensional FEM-based MonteCarlo simulation tool is used to accurately predict the planar motions of nano-fibers. In the near future, the Monte-Carlo simulation using three-dimensional FEM is expected to study the full motion of nano-fibers using Graphics Processing Unit (GPU) computing and High Performance Computing (HPC). 


\section{Chapter 5}

\section{Physical Understanding of Fiber-Fiber Interactions and Bounded Fluid Domain on Fiber Motion}

\section{$5.1 \quad$ Introduction}

This chapter investigates semi-dilute fiber suspensions in a bounded fluid domain for short-fiber reinforced composite materials processing. For industrial applications, the volume fraction of short fibers is large for semi-dilute and concentrated fiber suspensions. Therefore, fiber-fiber interactions affect fiber orientations and thus the rate of fiber alignment in the manufacturing processing. In this chapter, we study the semi-dilute fiber suspensions, i.e. the gap between fibers becomes closer, and hydrodynamic interactions becomes stronger, but the physical/mechanical contacts are still rare. Compared with the fiber size, the fluid domain of the polymer matrix is relatively large, so the unbounded assumption is valid for a single fiber motion. But the existence of many other fibers around one fiber reduces the fluid domain, so the study 
of fiber motion in a bounded fluid domain is critical in investigating the effect of semidilute or concentrated suspensions. Meanwhile, during the injection molding process, some fibers move close to one side of the mold cavity, which changes the behavior of fibers. Therefore, the direct use of Jeffery's equation [8] in the prediction of multiple fiber suspensions is not appropriate. Experiments have shown that fiber alignment occurs more slowly than that predicted by Jeffery-based models [30, 34]. Likewise, Stover et al. [77] experimentally measured fiber motions in semi-dilute suspensions, and found that fiber orientations are different from those in dilute suspensions. Additionally, the presence of a bounded wall could also change the rheological behavior of polymer matrix. Therefore, this chapter studies the semi-dilute fiber suspensions and the effect of a stationary wall on fiber orientations. The followings as related to fiber orientations in composite molding processes are studied:

- Hydrodynamic interaction between two fibers are modeled. With the presence of two fibers, the motion of one fiber affects its surrounding fluid which influences the dynamics of the other fiber, and vice versa.

- The effect of bounded fluid domain, which approximates the existence of many fibers surrounding a fiber, is investigated. With the presence of multiple fibers, the active fluid domain is bounded. The equivalent aspect ratio is proposed to quantify the fiber motion in a bounded fluid domain.

- Fiber motion near a stationary wall (mold cavity) is studied. The change of fiber motion and time period of fiber rotation near a close wall are observed in our simulation.

In this chapter, we physically address three critical issues in flow-induced fiber suspensions: fiber-fiber interactions, the effect of bounded fluid domain and the fiber motion near a stationary wall. We extend our earlier single fiber model (as seen in Chapter 3) to investigate hydrodynamic interactions between fibers, the effect of bounded fluid domain and the effect of a stationary wall. This approach computes 
the hydrodynamic forces and torques on fibers by solving governing equations of motion in fluid. The hydrodynamic forces and torques result from two scenarios: gross fluid motion and hydrodynamic interactions from other fibers. Our approach seeks fibers' velocities that zero the hydrodynamic torques and forces acting on the fibers by the surrounding fluid. Fiber motions are then computed using a RungeKutta approach to update fiber positions and orientations as functions of time. This method is quite general and allows for solving multiple fiber suspensions in complex fluids. Examples with fibers having various starting positions and orientations are considered and compared with Jeffery's single fiber solution. The possible reasons why fiber motions observed in experiments align slower than those predicted by Jeffery's theory are discussed.

As discussed in Chapter 1, a statistic approach using the probability function $\chi(\mathbf{P})$ is proposed by Folgar and Tucker III and a diffusion term $D_{r}$ is added to account for fiber-fiber interactions [10]. The orientation averaging using diffusionadded orientation distribution function yields the orientation tensor approach [12], which is applied regularly in industrial mold filling simulations. But the diffusion term $D_{r}$ includes the phenomenological parameter $C_{I}$, which has to be determined by fitting the experimental data. For different flow fields and flow conditions, $C_{I}$ is different and there is no guidelines to choose $C_{I}$.

In addition to the statistical method, continuum mechanics and physical modeling are used to study fiber-fiber interactions. Many researchers $[24,78,79]$ worked on the modification of stress constitutive equation to take into account the effect of fiber-fiber interactions on the total stress, and thus the fiber motions. But no constitutive equation is available in a closed form. In terms of the physical modeling, the inter-fiber hydrodynamic interactions are approximated and simulated using different methods. Mackaplow and Shaqfeh [80] and Rahnama and Shaqfeh [81] used the slender-body theory to describe the long-range hydrodynamic interactions. Ya- 
mane et al. [82] considered the short-range interactions between rod-like fibers using lubrication approximation. Fan et al. [37] applied the slender-body theory to approximate long-range hydrodynamic interactions and used lubrication approximation to calculate short-range interactions between fibers. Claeys and Brady [83] treated the hydrodynamic interactions from multiple other fibers as a single force and toque on the fiber.

In a similar manner, the existence of a bounded wall close to the fiber changes the velocity and pressure distribution around the fiber, and the physical behavior of fiber. Stover and Cohen [58] experimentally studied fiber motions near a bounded wall, and found that particles aligned with the flow direction and less than a particle half-length from a wall remain indefinitely. Jayageeth et al. [84] simulated the short fiber suspension in a bounded shear flow using stokesian dynamics simulation, and indicated that the time period of fiber rotation increases as the fiber moves closer to the wall.

\subsection{Methodology}

The methodology proposed in Chapter 3 is extended to consider more than one fiber. We use a finite element model to represent the fluid domain between multiple fibers and the outside bounded wall (cavity). Velocity and pressure distribution in the fluid domain are calculated using a Finite Element Method with the boundary conditions specified on fiber surfaces and the bounded wall. Our method searches fibers' linear and angular velocities, which zero the net forces and torques exerted by the

surrounding fluid on fibers. The forces and torques result from two scenarios: gross moving fluid and hydrodynamic interactions from other fibers. This method solves both long-range and short-range interactions (lubrication effect) simultaneously. 


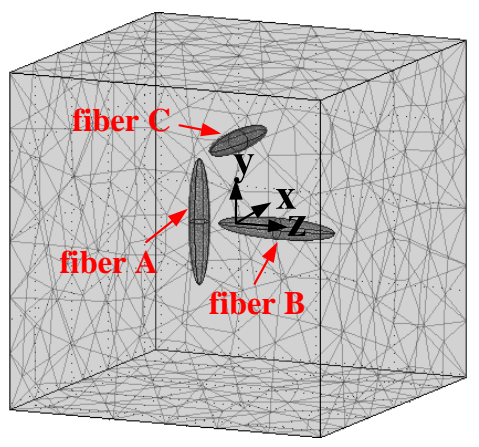

(a)



(b)



(c)

Figure 5.1: Finite element model of multiple fibers with boundary conditions

\subsubsection{Finite element model}

The governing equations that we solve is seen as Eq.(3.8). The fluid domain is modeled using meshes, as shown in Fig.5.1(a). The boundary conditions are specified on fiber surfaces and the outside bounded wall, as shown in Fig.5.1(b), in which BC3(A) refers to the boundary condition on fiber A. The boundary conditions are shown as:

(BC1) Velocity on the bounded wall (cavity):

$$
\mathbf{U}_{\mathrm{BC} 1}=\mathbf{U}_{0}
$$

(BC2) Pressure on the bounded wall (cavity):

$$
p_{\mathrm{BC} 2}=p_{0}
$$

(BC3) Velocity on the fiber surface (e.g., for fiber A):

$$
\mathrm{U}_{\mathrm{BC} 3(\mathrm{~A})}=\mathbf{U}_{\mathrm{c}}^{\mathrm{A}}+\boldsymbol{\omega}^{\mathrm{A}} \times \mathbf{r}
$$

where $\mathbf{U}_{0}$ is the undisturbed bulk flow, $p_{0}$ is the pressure benchmark, $\mathbf{r}$ is the position vector of point on fiber $\mathrm{A}, \mathbf{U}_{\mathrm{c}}^{\mathrm{A}}=\left[\dot{x}_{c}^{\mathrm{A}}, \dot{y}_{c}^{\mathrm{A}}, \dot{z}_{c}^{\mathrm{A}}\right]$ is the translation velocity of fiber A's centroid, and $\boldsymbol{\omega}^{\mathrm{A}}=\left[\omega_{x}^{\mathrm{A}}, \omega_{y}^{\mathrm{A}}, \omega_{z}^{\mathrm{A}}\right]^{T}$ is the angular velocity of fiber $\mathrm{A}$ in the $x y z$ 
coordinate system, defined as

$$
\boldsymbol{\omega}^{\mathrm{A}}=\left[\begin{array}{c}
\omega_{x}^{\mathrm{A}} \\
\omega_{y}^{\mathrm{A}} \\
\omega_{z}^{\mathrm{A}}
\end{array}\right]=\left[\begin{array}{c}
\dot{\phi}^{\mathrm{A}}+\dot{\psi}^{\mathrm{A}} \cos \theta^{\mathrm{A}} \\
-\dot{\theta}^{\mathrm{A}} \sin \phi^{\mathrm{A}}+\dot{\psi}^{\mathrm{A}} \sin \theta^{\mathrm{A}} \cos \phi^{\mathrm{A}} \\
\dot{\theta} \cos \phi^{\mathrm{A}}+\dot{\psi}^{\mathrm{A}} \sin \theta \sin \phi^{\mathrm{A}}
\end{array}\right]
$$

where $\phi, \theta$ and $\psi$ are defined in Fig.3.3, which is the same as those used in Jeffery's paper [8]. The boundary condition specified on the surface of fiber $\mathrm{A}$ is defined by the translational velocity of fiber A's centroid and its angular velocity. In a similar manner, for fibers B, C and so on, the velocity boundary conditions BC3(B), BC3(C), etc. can be specified. As discussed in Chapter 3, the system of equations are partitioned as

$$
\left[\begin{array}{cc}
\mathbf{K}_{f f} & \mathbf{K}_{f e} \\
\mathbf{K}_{e f} & \mathbf{K}_{e e}
\end{array}\right]\left[\begin{array}{c}
\mathbf{V}_{f} \\
\mathbf{V}_{e}
\end{array}\right]=\left[\begin{array}{c}
\mathbf{F}_{f} \\
\mathbf{F}_{e}
\end{array}\right]
$$

It follows that velocity and pressure distribution $\mathbf{V}_{f}$ in the fluid domain is calculated from Eq.(3.14).

\subsubsection{Hydrodynamic forces and torques}

With the calculated fluid velocity and pressure, the reaction force $\mathbf{F}_{e}$ on the essential nodes (including the nodes on the surfaces of all suspended fibers) can be calculated from Eq.(3.15). Therefore, the force vector exerted by the fluid on fiber A is obtained by summing up the reaction forces at the nodes on fiber A surface using

$$
\mathbf{F}_{\mathrm{A}}=-\sum_{n=1}^{N_{\mathrm{A}}}\left(\mathbf{F}_{e}\right)_{n}
$$

where $N_{A}$ is the total number of nodes on fiber A's surface. The torque on fiber A is 
obtained by summing up the cross product of position vector $\mathbf{r}$ and reaction force $\mathbf{F}_{e}$ at the nodes located on fiber A's surface as

$$
\mathbf{T}_{\mathrm{A}}=-\sum_{n=1}^{N_{\mathrm{A}}}\left(\mathbf{r} \times \mathbf{F}_{e}\right)_{n}
$$

Likewise, force and torque exerted on other fibers can be obtained. In a simple shear flow, a single fiber performs a periodic tumbling motion within the moving fluid. When multiple fibers suspend in the fluid, the hydrodynamic interactions between fibers come into play. Specifically, the motion of one fiber affects its surrounding fluid, and in turn influences the motions of other fibers, and vice versa. Therefore, the force and torque on fiber A depend on fiber A's velocities and the motions of other fibers. The force and torque on fiber A can be represented as

$$
\begin{aligned}
& \mathbf{F}^{\mathrm{A}}=\mathbf{F}^{\mathrm{A}}\left(\dot{x}_{c}^{\mathrm{A}}, \dot{y}_{c}^{\mathrm{A}}, \dot{z}_{c}^{\mathrm{A}}, \dot{\phi}^{\mathrm{A}}, \dot{\theta}^{\mathrm{A}}, \dot{\psi}^{\mathrm{A}}, \dot{x}_{c}^{\mathrm{B}}, \dot{y}_{c}^{\mathrm{B}}, \dot{z}_{c}^{\mathrm{B}}, \dot{\phi}^{\mathrm{B}}, \dot{\theta}^{\mathrm{B}}, \dot{\psi}^{\mathrm{B}}, \cdots\right) \\
& \mathbf{T}^{\mathrm{A}}=\mathbf{T}^{\mathrm{A}}\left(\dot{x}_{c}^{\mathrm{A}}, \dot{y}_{c}^{\mathrm{A}}, \dot{z}_{c}^{\mathrm{A}}, \dot{\phi}^{\mathrm{A}}, \dot{\theta}^{\mathrm{A}}, \dot{\psi}^{\mathrm{A}}, \dot{x}_{c}^{\mathrm{B}}, \dot{y}_{c}^{\mathrm{B}}, \dot{z}_{c}^{\mathrm{B}}, \dot{\phi}^{\mathrm{B}}, \dot{\theta}^{\mathrm{B}}, \dot{\psi}^{\mathrm{B}}, \cdots\right)
\end{aligned}
$$

The mass and mass moment of inertia of small suspended fibers are neglected (the same as Jeffery's theory), so the resultant forces and torques on all fibers are always zero at each time instant. The linear and angular velocities of all fibers are calculated by solving the following system of equations,

$$
\left\{\begin{array}{c}
\mathbf{F}^{\mathrm{A}}\left(\dot{x}_{c}^{\mathrm{A}}, \dot{y}_{c}^{\mathrm{A}}, \dot{z}_{c}^{\mathrm{A}}, \dot{\phi}^{\mathrm{A}}, \dot{\theta}^{\mathrm{A}}, \dot{\psi}^{\mathrm{A}}, \dot{x}_{c}^{\mathrm{B}}, \dot{y}_{c}^{\mathrm{B}}, \dot{z}_{c}^{\mathrm{B}}, \dot{\phi}^{\mathrm{B}}, \dot{\theta}^{\mathrm{B}}, \dot{\psi}^{\mathrm{B}}, \cdots\right)=\mathbf{0} \\
\mathbf{T}^{\mathrm{A}}\left(\dot{x}_{c}^{\mathrm{A}}, \dot{y}_{c}^{\mathrm{A}}, \dot{z}_{c}^{\mathrm{A}}, \dot{\phi}^{\mathrm{A}}, \dot{\theta}^{\mathrm{A}}, \dot{\psi}^{\mathrm{A}}, \dot{x}_{c}^{\mathrm{B}}, \dot{y}_{c}^{\mathrm{B}}, \dot{z}_{c}^{\mathrm{B}}, \dot{\phi}^{\mathrm{B}}, \dot{\theta}^{\mathrm{B}}, \dot{\psi}^{\mathrm{B}}, \cdots\right)=\mathbf{0} \\
\mathbf{F}^{\mathrm{B}}\left(\dot{x}_{c}^{\mathrm{A}}, \dot{y}_{c}^{\mathrm{A}}, \dot{z}_{c}^{\mathrm{A}}, \dot{\phi}^{\mathrm{A}}, \dot{\theta}^{\mathrm{A}}, \dot{\psi}^{\mathrm{A}}, \dot{x}_{c}^{\mathrm{B}}, \dot{y}_{c}^{\mathrm{B}}, \dot{z}_{c}^{\mathrm{B}}, \dot{\phi}^{\mathrm{B}}, \dot{\theta}^{\mathrm{B}}, \dot{\psi}^{\mathrm{B}}, \cdots\right)=\mathbf{0} \\
\mathbf{T}^{\mathrm{B}}\left(\dot{x}_{c}^{\mathrm{A}}, \dot{y}_{c}^{\mathrm{A}}, \dot{z}_{c}^{\mathrm{A}}, \dot{\phi}^{\mathrm{A}}, \dot{\theta}^{\mathrm{A}}, \dot{\psi}^{\mathrm{A}}, \dot{x}_{c}^{\mathrm{B}}, \dot{y}_{c}^{\mathrm{B}}, \dot{z}_{c}^{\mathrm{B}}, \dot{\phi}^{\mathrm{B}}, \dot{\theta}^{\mathrm{B}}, \dot{\psi}^{\mathrm{B}}, \cdots\right)=\mathbf{0} \\
\cdots \cdots
\end{array}\right.
$$

If there are two fibers (A and $\mathrm{B}$ ) in the fluid, there are twelve unknowns and twelve force/torque equilibrium equations $\left(\mathbf{F}=\left[F_{x}, F_{y}, F_{z}\right]\right.$ and $\left.\mathbf{T}=\left[T_{x}, T_{y}, T_{z}\right]\right)$. There- 
fore, the velocities of fibers at each time instant are solved using a Newton-Raphson iteration algorithm.

\subsubsection{Updating fiber orientations and positions}

At each time step $t_{i}$, provided that fiber positions and orientations are given, the fiber velocities are obtained by solving Eq.(5.9) to give $\dot{\mathbf{y}}_{i}=\mathbf{f}\left(t_{i}, \mathbf{y}_{i}\right)$, where $\mathbf{y}=$ $\left[x_{c}^{\mathrm{A}}, y_{c}^{\mathrm{A}}, z_{c}^{\mathrm{A}}, \phi^{\mathrm{A}}, \theta^{\mathrm{A}}, \psi^{\mathrm{A}}, x_{c}^{\mathrm{B}}, y_{c}^{\mathrm{B}}, z_{c}^{\mathrm{B}}, \phi^{\mathrm{B}}, \theta^{\mathrm{B}}, \psi^{\mathrm{B}}, \cdots\right]$. With the specified time interval $\Delta t$, we apply the $4^{\text {th }}$-order Runge-Kutta algorithm to update fiber orientations and centroid positions in the next time time step $t_{i+1}\left(=t_{i}+\triangle t\right)$, as seen in Eq.(2.14). As fibers move, the relative position and orientation between multiple fibers change. Therefore, the re-meshing process is used for the modeling of multiple fiber suspensions in the next time step.

\subsection{Effect of the bounded fluid domain}

Experiments have shown that the fiber motion is observed to have a longer period than that predicted by Jeffery's equation. One possible explanation is the existence of objects around fibers, such as the mold cavity and other fibers, while a single fiber motion in an infinite fluid domain is studied in Jeffery's theory. In pure Jeffery's equation, fiber performs the periodic tumbling motion continuously. But Eq.(1.16) gives an fiber alignment along the flow direction with the consideration of fiberfiber interactions defined by the $D_{r}$ term. Therefore, fiber-fiber interactions have a tremendously effect on the fiber behavior. The existence of multiple fibers around a fiber reduces the fluid domain surrounding that fiber, which is modeled as the fiber motion in a bounded fluid domain, as seen in Fig.5.2.

The effect of reduced fluid domain is still quantified by the equivalent aspect ratio (denoted as $r_{e}^{*}$ ) of fiber. In Ref.[85], Zhang et al. used Cox's [41] method to 

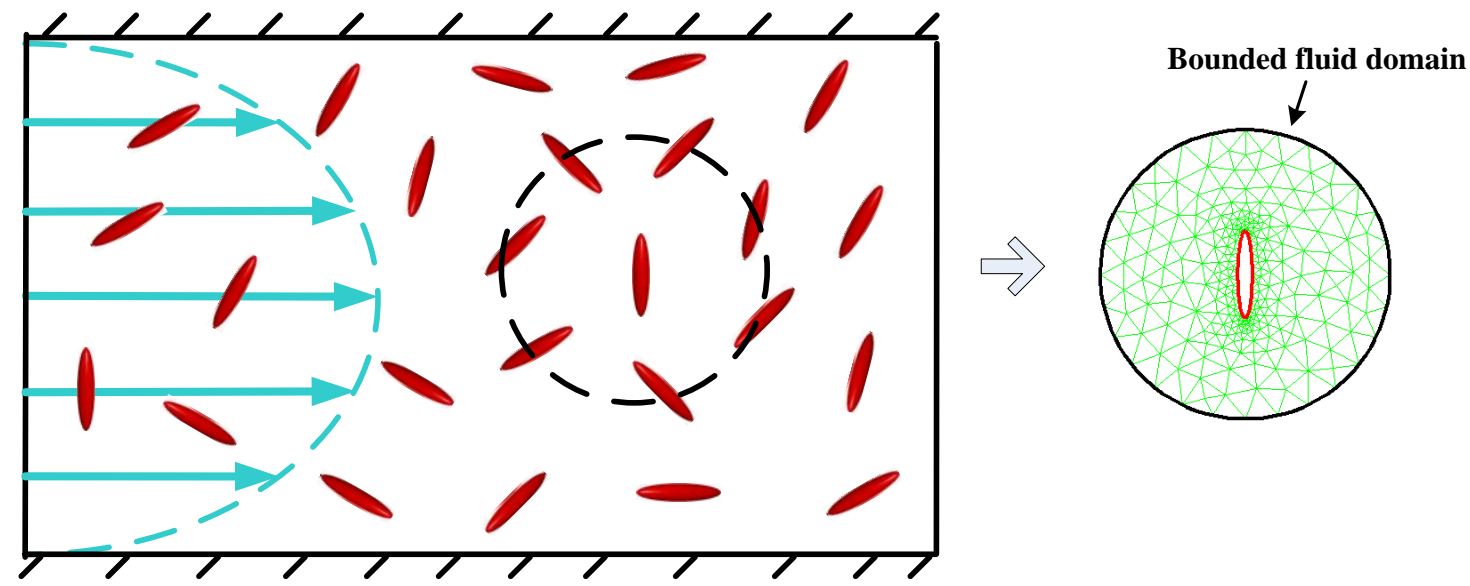

Figure 5.2: The effect of multiple fiber interactions

numerically generate the equivalent aspect ratios of any axisymmetric fibers including cylindrical and bead-chain fibers. In this chapter, the proposed equivalent aspect ratio is still used to quantify the effect of reduced fluid domain. More importantly, the equivalent aspect ratio is not only a function of fiber shape, but also relies on the size of the fluid domain. By evaluating the torques on fiber when fiber is fixed in two directions as seen in Fig.5.3, the equivalent aspect ratio is calculated as [85]
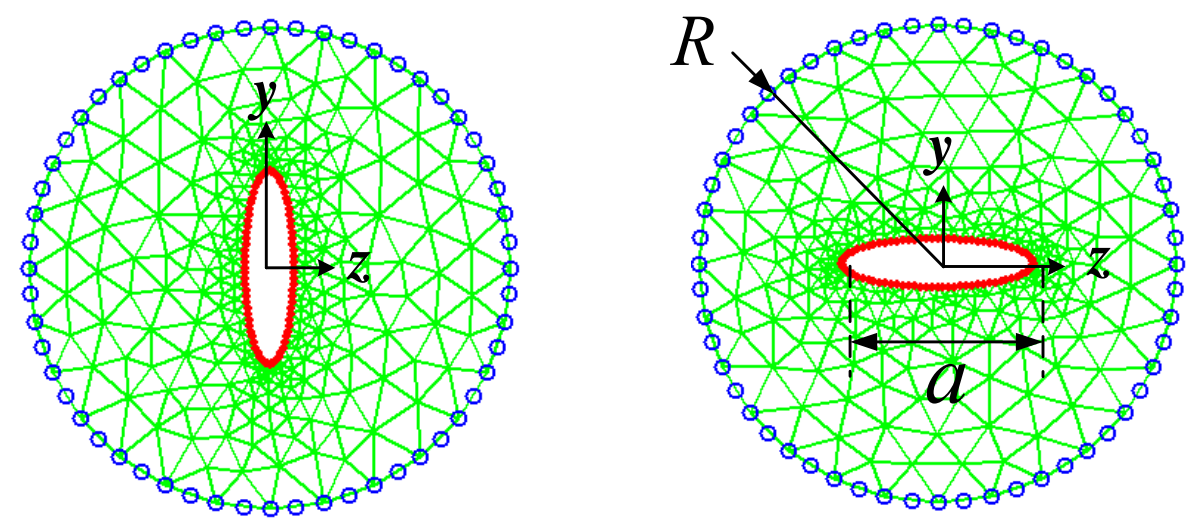

Figure 5.3: (left) Fiber is fixed in the vertical direction $(\phi=0)$; (right) Fiber is fixed in the horizontal direction $(\phi=\pi / 2)$

$$
r_{e}^{*}=\sqrt{\frac{T_{V}}{T_{H}}}
$$

where $T_{V}$ and $T_{H}$ represent the torques when the fiber is fixed in the vertical and hor- 
izontal directions, respectively. In the presented simulations, we use a dimensionless parameter $\varepsilon$ to quantify the effect of fluid domain, defined as

$$
\varepsilon=\frac{2 R}{a}
$$

where $a$ is the long axis of an ellipsoidal fiber, or the length of a cylindrical fiber, and $R$ is the radius of the spherical fluid domain in terms of fiber centroid, as seen in Fig.5.3. In Jeffery's theory, the fiber moves in an unbounded fluid, i.e., $R=\infty$ and $\varepsilon=\infty$. However, the bounded fluid domain would change the fluid velocity and pressure distribution in the bulk fluid. This has been approved in a similar problem that a creeping uniform flow passes a fixed sphere in an unbounded domain $[86,87]$, seen in Fig.5.4. The exact solution to that problem is [87]

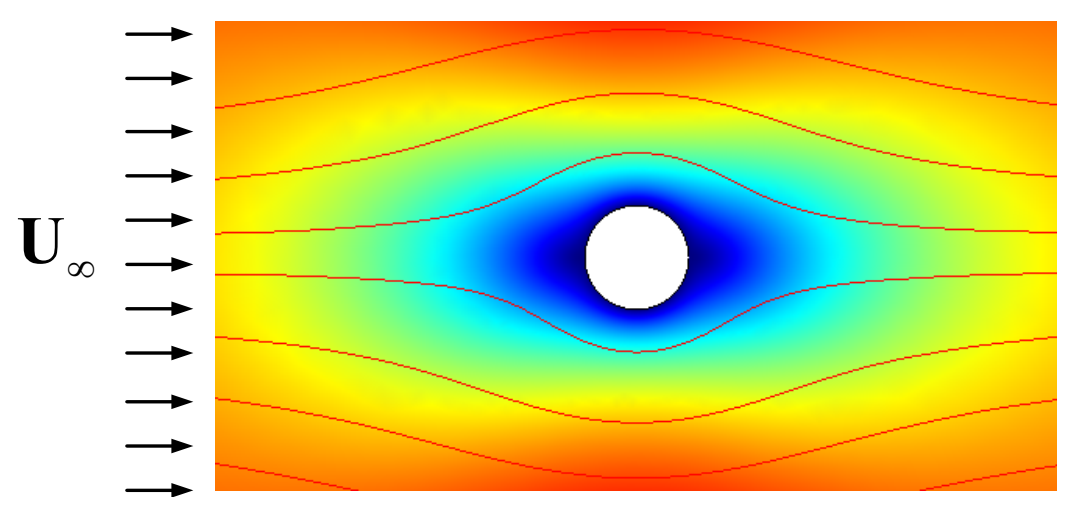

Figure 5.4: Uniform flow passes a fixed sphere in an unbounded fluid domain

$$
\mathbf{U}=\mathbf{U}_{\infty}-\frac{3 a}{8}\left[\frac{\mathbf{U}_{\infty}}{r}+\frac{\mathbf{r} \mathbf{U}_{\infty} \cdot \mathbf{r}}{r^{3}}\right]-\frac{a^{3}}{32}\left[\frac{\mathbf{U}_{\infty}}{r^{3}}-3 \frac{\mathbf{r} \mathbf{U}_{\infty} \cdot \mathbf{r}}{r^{5}}\right]
$$

where $a$ is the diameter of the spherical fiber, $\mathbf{U}_{\infty}$ is the undisturbed uniform fluid, $\mathbf{r}$ is the position vector in space in terms of the sphere centroid, and $r$ is the magnitude of the position vector $\mathbf{r}$. From the exact solution of velocity distribution, we can see that at the position where $r=5 a, \mathbf{U}$ differs from $\mathbf{U}_{\infty}$ by about $8 \%$. That is to say, if $\mathbf{U}_{\infty}$ is specified at $r=5 a$, i.e. $\varepsilon=2 r / a=10$, the velocity deviates 
from $\mathbf{U}$ in Eq.(5.12). Therefore, in order to approximate the unbounded condition using the proposed Finite Element Method, the size of the fluid domain should be at least 20 times larger than the fiber size. In an analogous manner, for multiple fiber suspensions, the presence of dense fibers reduces the fluid domain around the fiber, changes the fluid velocity and pressure, and in turn disturbs the fiber motion. In the proposed methodology, we specify the undisturbed simple shear flow on the bounded wall, which has a finite distance from the fiber, so $\varepsilon \neq \infty$. In Chapter 3 , we showed that when $\varepsilon=40$, our results to solve a single fiber motion within a viscous fluid are in excellent agreement with Jeffery's solution. In this section, we study the effect of different $\varepsilon^{\prime} s$ on fiber orientation using the proposed Finite Element Method, because the existence of multiple fibers reduces the active fluid domain.

\subsection{Implementation and examples}

For the modeling of fiber-fiber interactions, the re-meshing process is required to model the relative orientation/position of two fibers. The re-meshing process for three-dimensional finite element model is time-consuming and tedious, so a twodimensional finite element model is used to study fiber-fiber interaction between two fibers. It has been demonstrated in Chapter 4 that for the planar fiber motion, i.e. fiber only moves in a plane, two-dimensional FEM can also accurately predict the dynamics of fiber within a viscous fluid and save huge computation time compared with the three-dimensional FEM.

\subsubsection{Fiber-fiber interaction between two fibers}

In this section, we investigate the effect of fiber-fiber interactions on fiber motions. We only consider two fibers so as to physically understand the mutual hydrodynamic

effect. The motion of one fiber disturbs the local fluid velocity and pressure around 
fiber, and then affects the motions of other fibers nearby. The hydrodynamic effects from other fibers are categorized into two groups: long-range and short-range hydrodynamic effects, which are solved together using the proposed methodology. In this section, various examples with different distances between fiber centroids are presented. In order to quantify the effect of hydrodynamic effect between two fibers, we propose to use the parameter $\Im$, defined as the ratio of the distance between fiber centroids to the fiber size as

$$
\Im=\frac{d}{a}
$$

where $d$ is the distance between the centroids of two fibers, as seen in Fig.5.5. When $d \gg a$, the long-range hydrodynamic interactions come into play, and when $d$ is close to $a$, the short-range hydrodynamic interactions dominate.

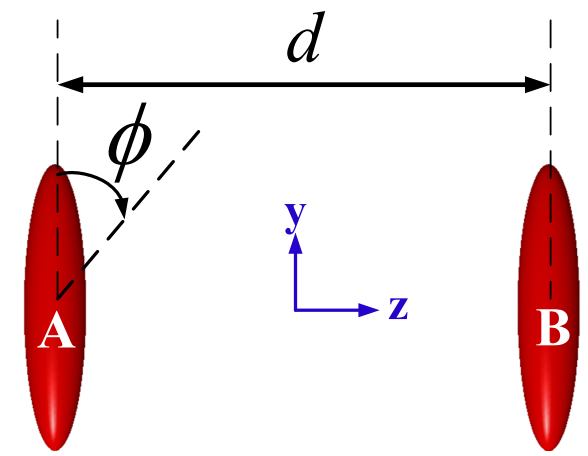

(a)

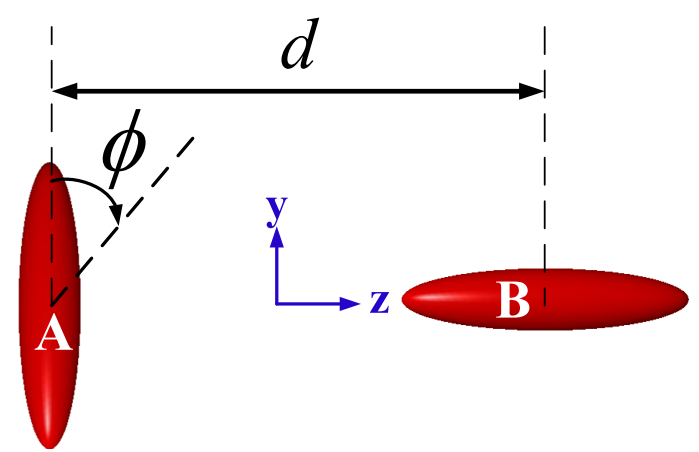

(b)

Figure 5.5: Initial configurations of two fibers

\section{Fibers stay far away from each other}

When two fibers are far away, i.e. $d \gg a$ and $\Im \gg 1$, the hydrodynamic interaction is trivial, which cannot visibly affect fiber motions. Here, we model two far-away ellipsoidal fibers in the $y z$ plane, with the initial configuration shown in Fig.5.5(a) and the dimensionless simulation parameters are tabulated in Table 5.1.

The fiber motions in one period are shown in Fig.5.6, from which we can see that 


\begin{tabular}{|c|c|}
\hline Parameters & Values \\
\hline \hline fiber size & $a=3$ and $b=1$, so $r_{e}=3$ \\
\hline initial orientation & $\phi^{A}=\phi^{B}=0$ \\
\hline initial position & $y_{c}^{A}=y_{c}^{B}=0, z_{c}^{A}=-20, y_{c}^{B}=20$, so $\Im \gg 1$ \\
\hline properties of bulk fluid & viscosity: $\mu=1$, Shear flow: $U_{z}=\dot{\gamma} y$, where $\dot{\gamma}=1$ \\
\hline
\end{tabular}

Table 5.1: Parameters to simulate the motions of two ellipsoidal fibers
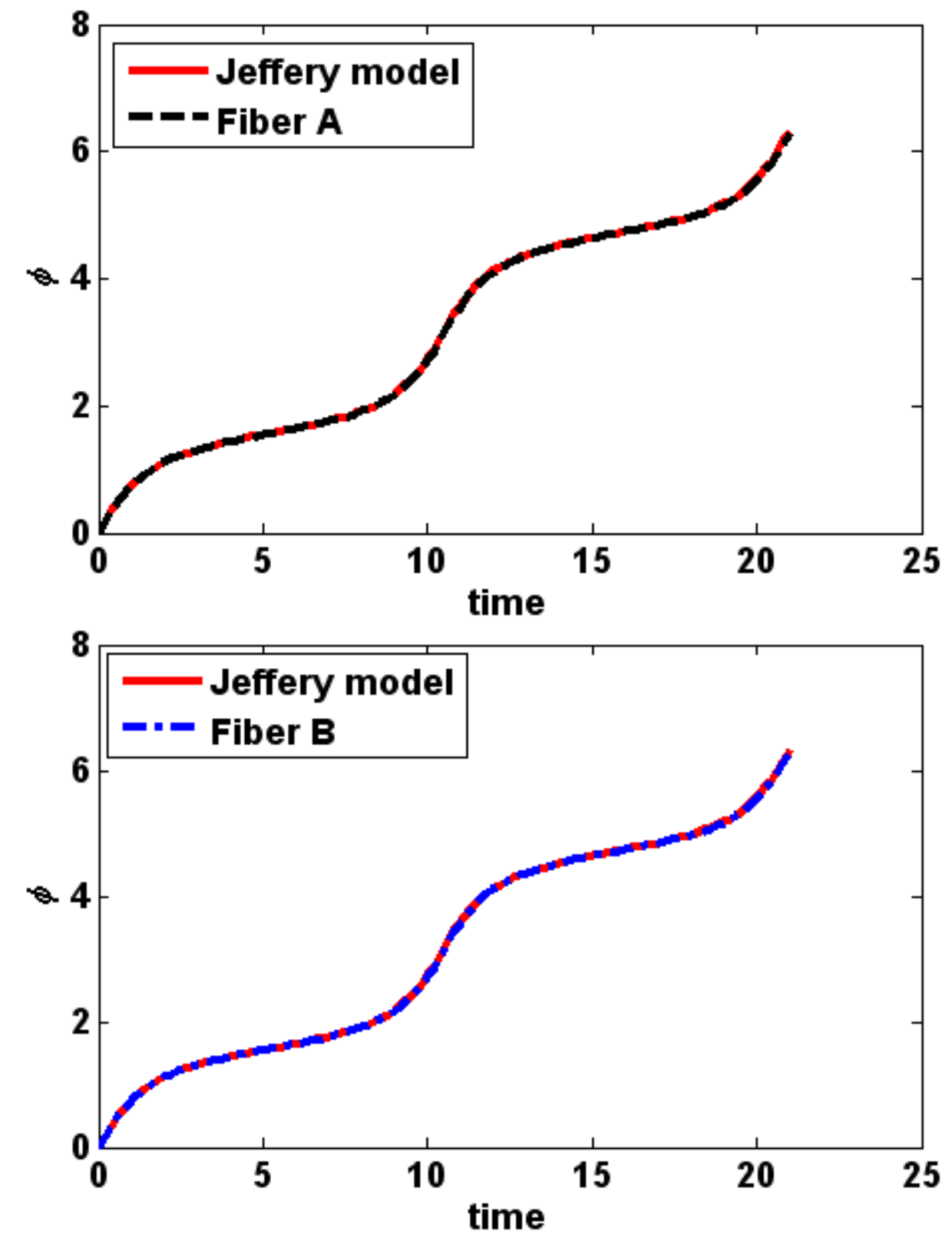

Figure 5.6: Fiber orientations of two far-away ellipsoidal fibers $(\Im \gg 1)$ 
both fibers remain in the Jeffery's orbit, i.e. the hydrodynamic interaction between two fibers is trivial, when two fibers stay far away from each other $(\Im \gg 1)$.

\section{Fibers stay close to each other}

When $\Im$ is comparable with or less than 1 , the hydrodynamic interaction between two fibers becomes stronger. In this section, we give three examples to model the hydrodynamic interaction between two close fibers, and study the hydrodynamic effect on fiber motions as two fibers move closer to each other.

Example 1: Two vertical ellipsoidal fibers with $\Im=0.5$

We use the same simulation parameters, but decrease the distance between two fibers' centroids to be 1.5. By putting the two fibers closer, the short-range hydrodynamic interaction takes effect and changes the behavior of two fibers, with the fiber orientations shown in Fig.5.7.

With the presence of two fibers close to each other, fiber motions are slowed down, with the time period compared with that when fibers stay away from each other in Table 5.2, from which we find that the short-range hydrodynamic interaction slows down fiber motions. Therefore, we posit that the slow motion observed in Refs. [30] and [34] could result from fiber-fiber interactions in semi-dilute suspensions.

\begin{tabular}{|c|c|c|c|}
\hline Scenarios & $\begin{array}{c}\text { Two close } \\
\text { ellipsoids }\end{array}$ & $\begin{array}{c}\text { Two far-away } \\
\text { ellipsoids }\end{array}$ & $\begin{array}{c}\text { Relative } \\
\text { difference }\end{array}$ \\
\hline \hline Time period & 21.15 & 23.3 & $10 \%$ \\
\hline
\end{tabular}

Table 5.2: Comparison of time periods of fiber rotations.

The linear motions of two fibers are also investigated using the proposed FEM, with the results shown in Fig.5.8, from which it can be seen that two fibers do not stay in the original positions, but have the relative motion with each other, which results from the mutual hydrodynamic interaction though the bulk fluid.

In order to physically understand the hydrodynamic interactions between two 

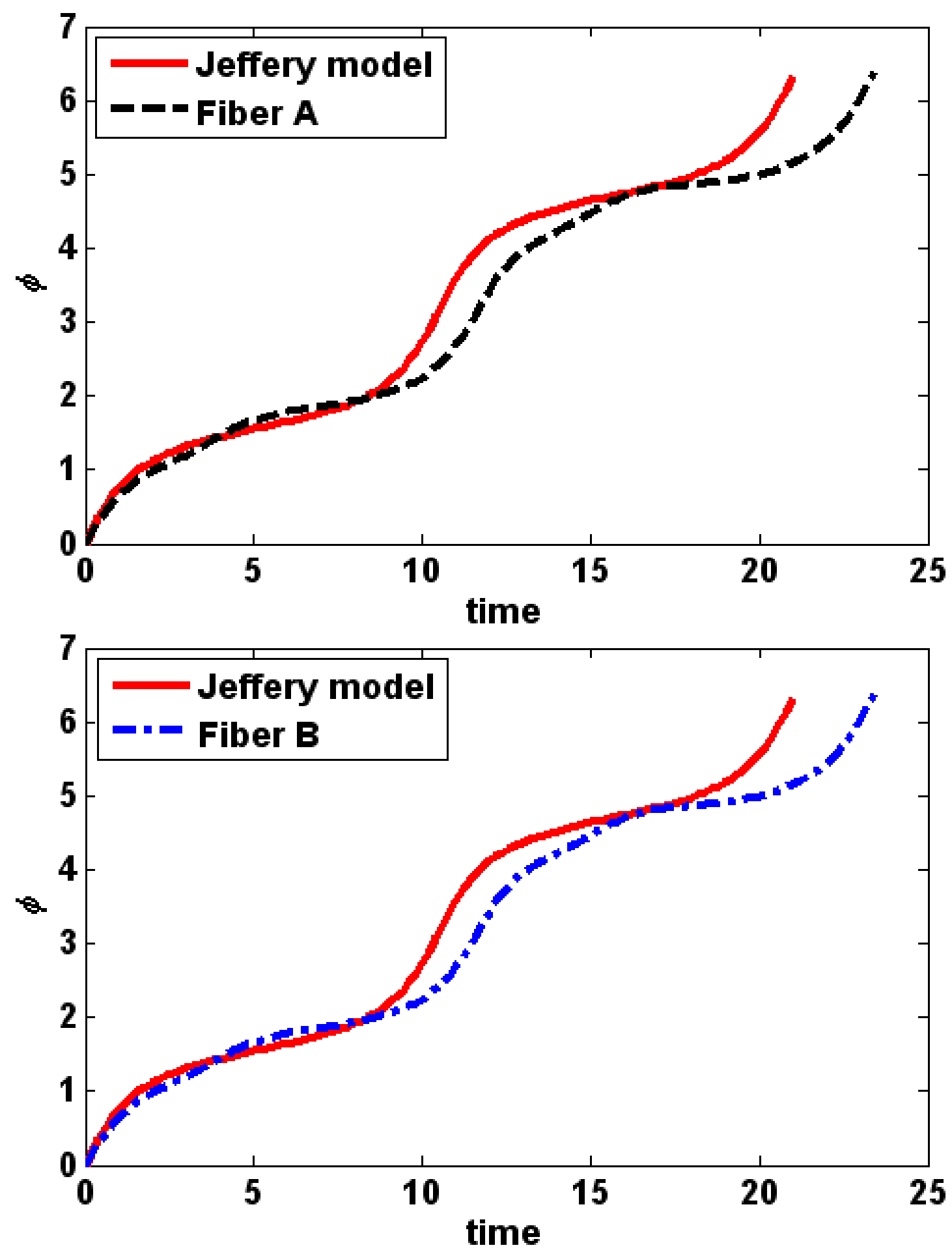

Figure 5.7: Fiber orientations of two close vertical ellipsoidal fibers $(\Im=0.5)$

fibers, we look into the local velocity and pressure distribution of bulk fluid around the fibers. Here we investigate the fluid properties at the initial orientation/position 

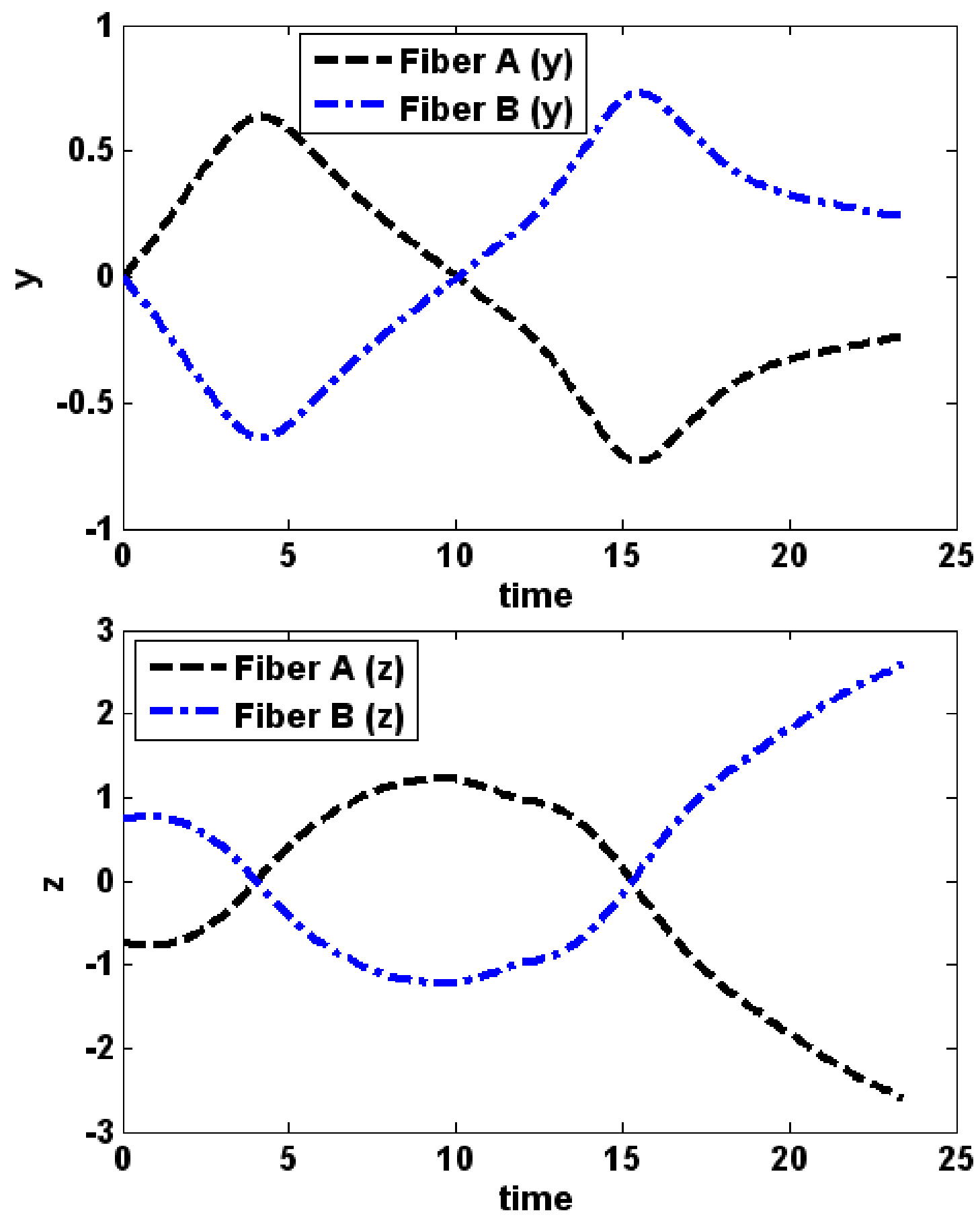

Figure 5.8: Fiber translations of two close vertical ellipsoidal fibers $(\Im=0.5)$

$\left(t_{i}=0\right)$ for a single fiber and two fibers, as seen in Fig.5.9, from which it can be seen that the existence of two fibers changes the local fluid properties, such as the active shear rate of fluid around fiber, and then changes the fiber motions. 


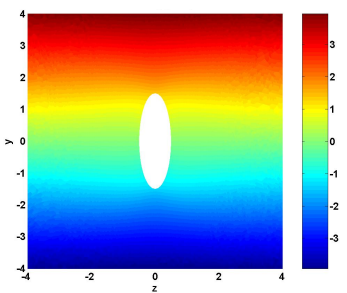

(a) z velocity

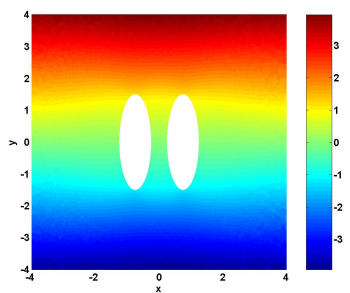

(e) z velocity

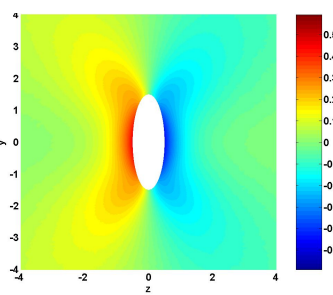

(b) y velocity

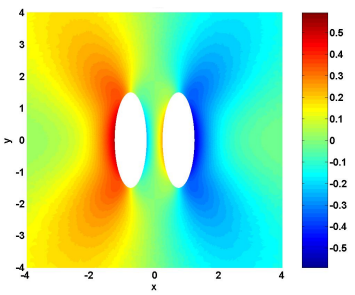

(f) y velocity

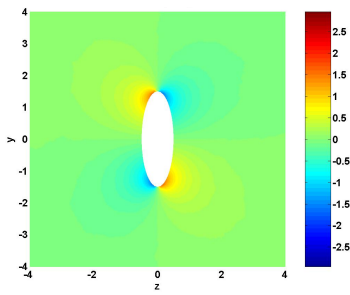

(c) pressure

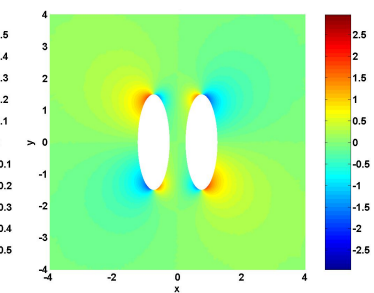

(g) pressure

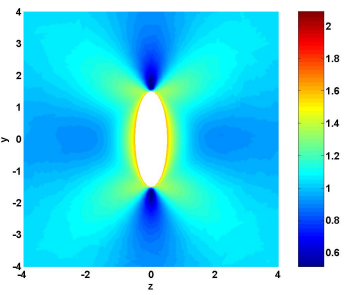

(d) shear rate

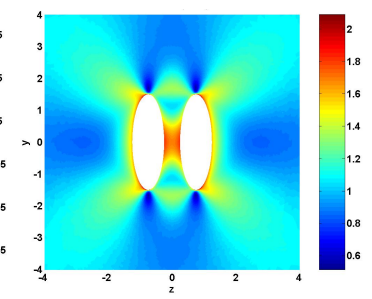

(h) shear rate

Figure 5.9: Disturbance of fiber motion on fluid velocity, pressure and shear rate around fiber surface at $t_{i}=0$

Example 2: One vertical and one horizontal fibers with $\Im=1$

In this example, we investigate the motions of two fibers, but with different starting orientations and positions. The initial orientations of two fibers are $\phi^{A}=0, \phi^{B}=$ $\pi / 2$, and the initial positions are $y_{c}^{A}=y_{c}^{B}=0, z_{c}^{A}=-1.5, z_{c}^{B}=1.5$, as seen in Fig.5.5(b). So the parameter $\Im$ equals 1. All other parameters are the same as those in Example 1. The plot of fiber orientations in one complete period is shown in Fig.5.10, from which we find that the short-range hydrodynamic interaction moves the fibers off Jeffery's trajectory, and slows down fiber motions. In this example, fiber A is initially vertical, and moves faster than fiber B, which is initially horizontal. When fiber A approaches fiber $\mathrm{B}$ at $t_{i}=5$, the separation between two fibers becomes small, and the hydrodynamic interaction becomes stronger. So the motions of both fibers are retarded, and then two fibers has a physical contact at $t_{i}=17.4$. After the collision, multiple fibers become a floc, and move together, which is beyond the scope of this chapter.

In a similar manner, we keep track of the trajectories of fibers' centroids, shown in Fig.5.11, from which it can be seen that two fibers do not translate with the 

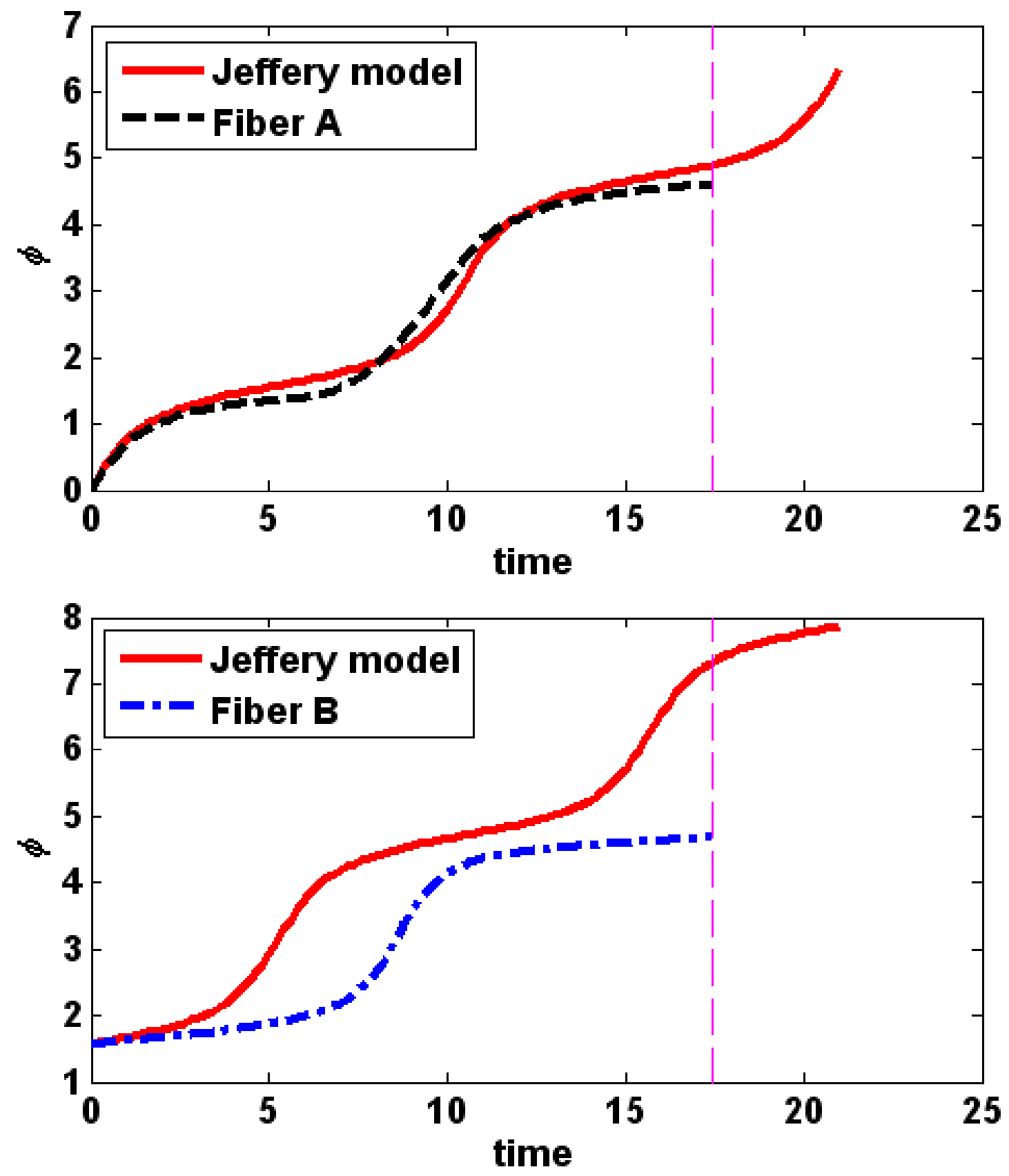

Figure 5.10: Fiber orientations of one vertical fiber and one horizontal fiber $(\Im=1)$ undisturbed simple shear flow evaluated at fiber centroids. Instead, fibers have upand-down motions. 

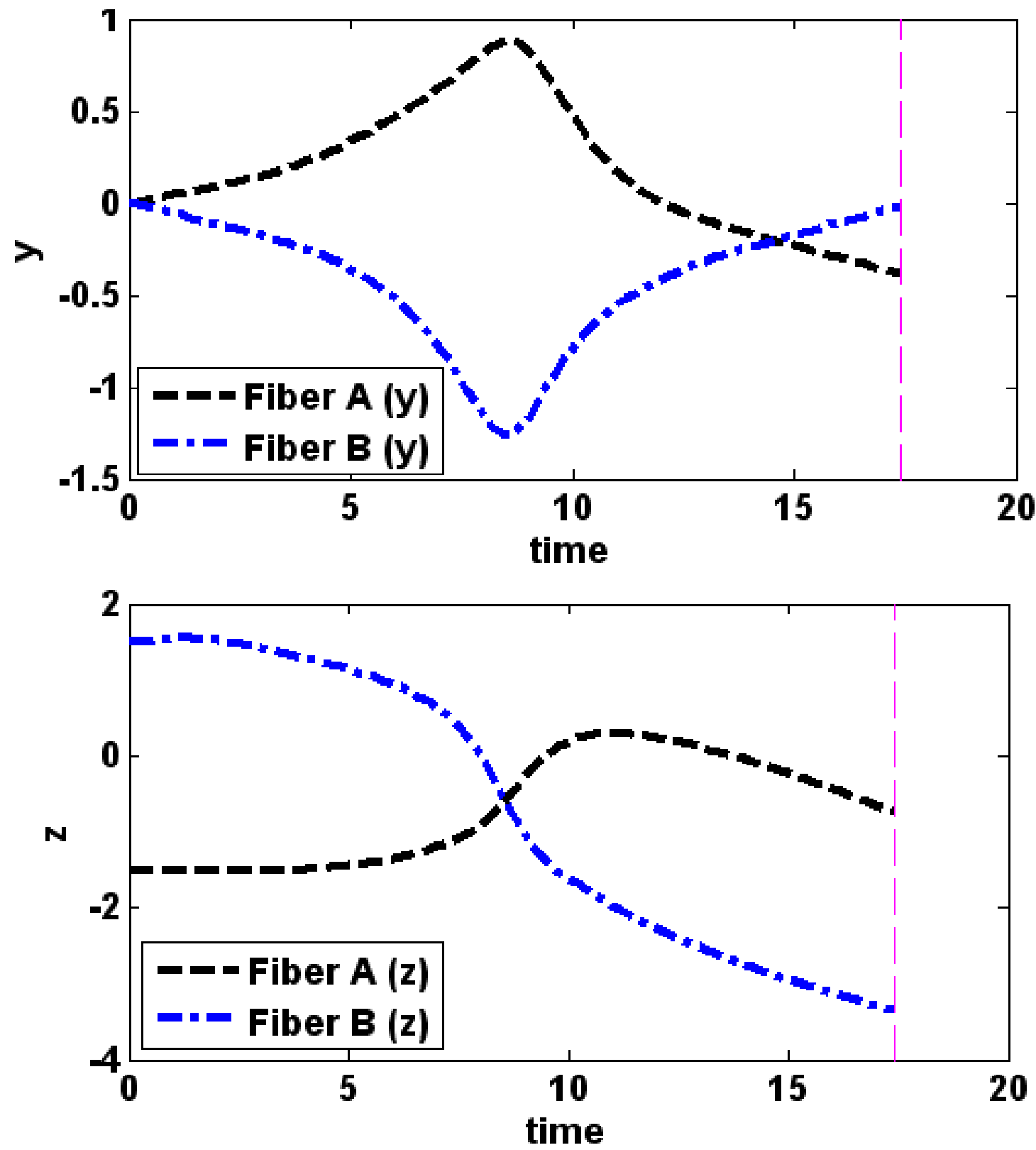

Figure 5.11: Fiber translations of one vertical fiber and one horizontal fiber $(\Im=1)$

Example 3: Two longer ellipsoidal fibers with $\Im=0.25$

This example is presented to investigate the effect of fiber's aspect ratio on the hydrodynamic interaction between two fibers. In Example 1, the geometric aspect ratios of two fibers are 3 , and two fibers are initially in the vertical position with the centroid separation to be 1.5. In this example, we use two ellipsoids with a larger 

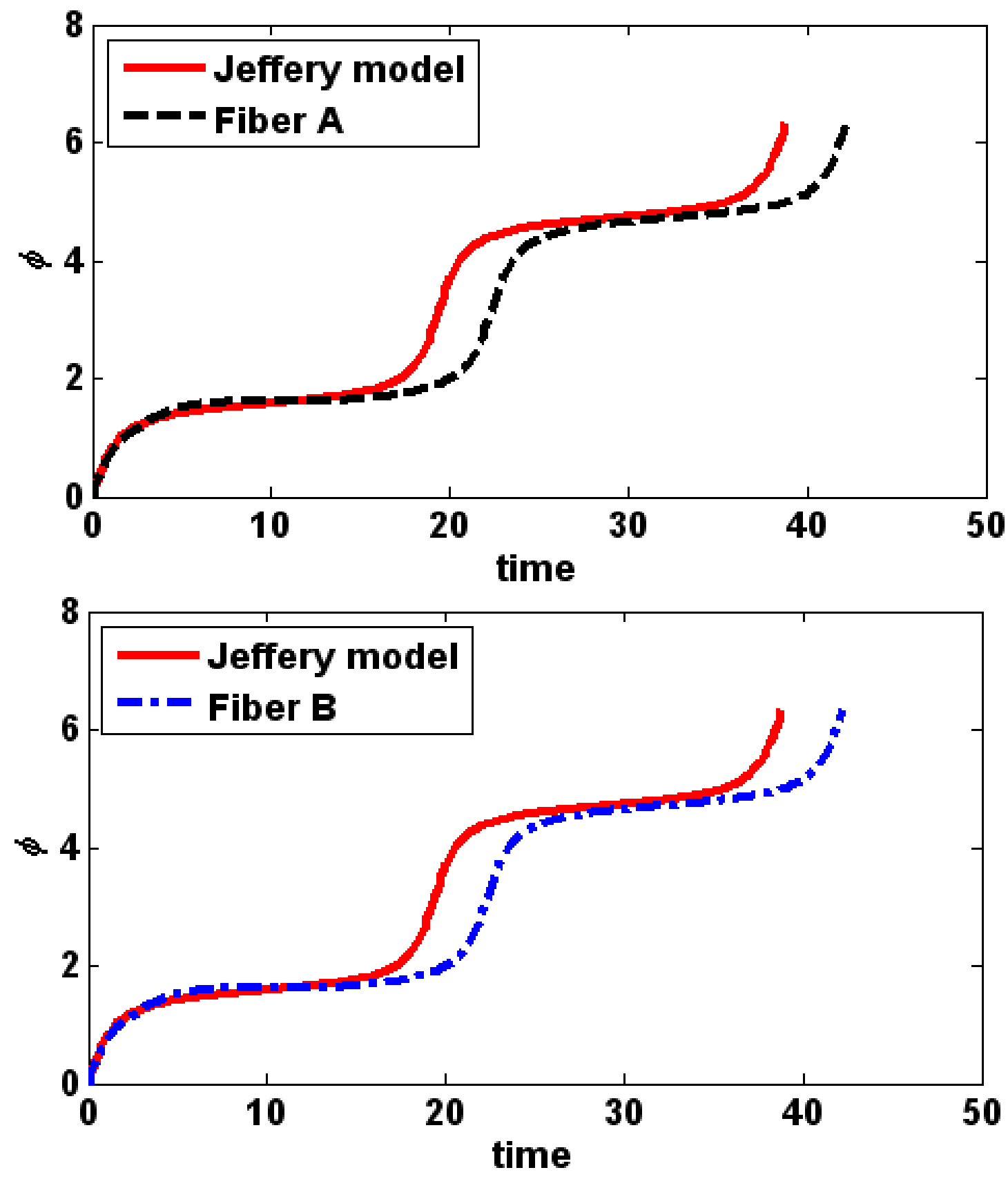

Figure 5.12: Fiber orientations of two long ellipsoidal fibers $(\Im=0.25)$

geometric aspect ratios $\left(a=6, b=1, r_{e}=6\right)$, but keep the same centroid separation. The initial configurations of two fibers are: $\phi^{A}=\phi^{B}=0$ and $y_{c}^{A}=y_{c}^{B}=0, z_{c}^{A}=$ $-0.75, z_{c}^{B}=0.75$. So $d=1.5$, and $\Im=0.25<1$. Fiber positions and orientations in one period are shown in Fig.5.12. We see that fiber motions apparently deviate from Jeffery's single fiber solution. 

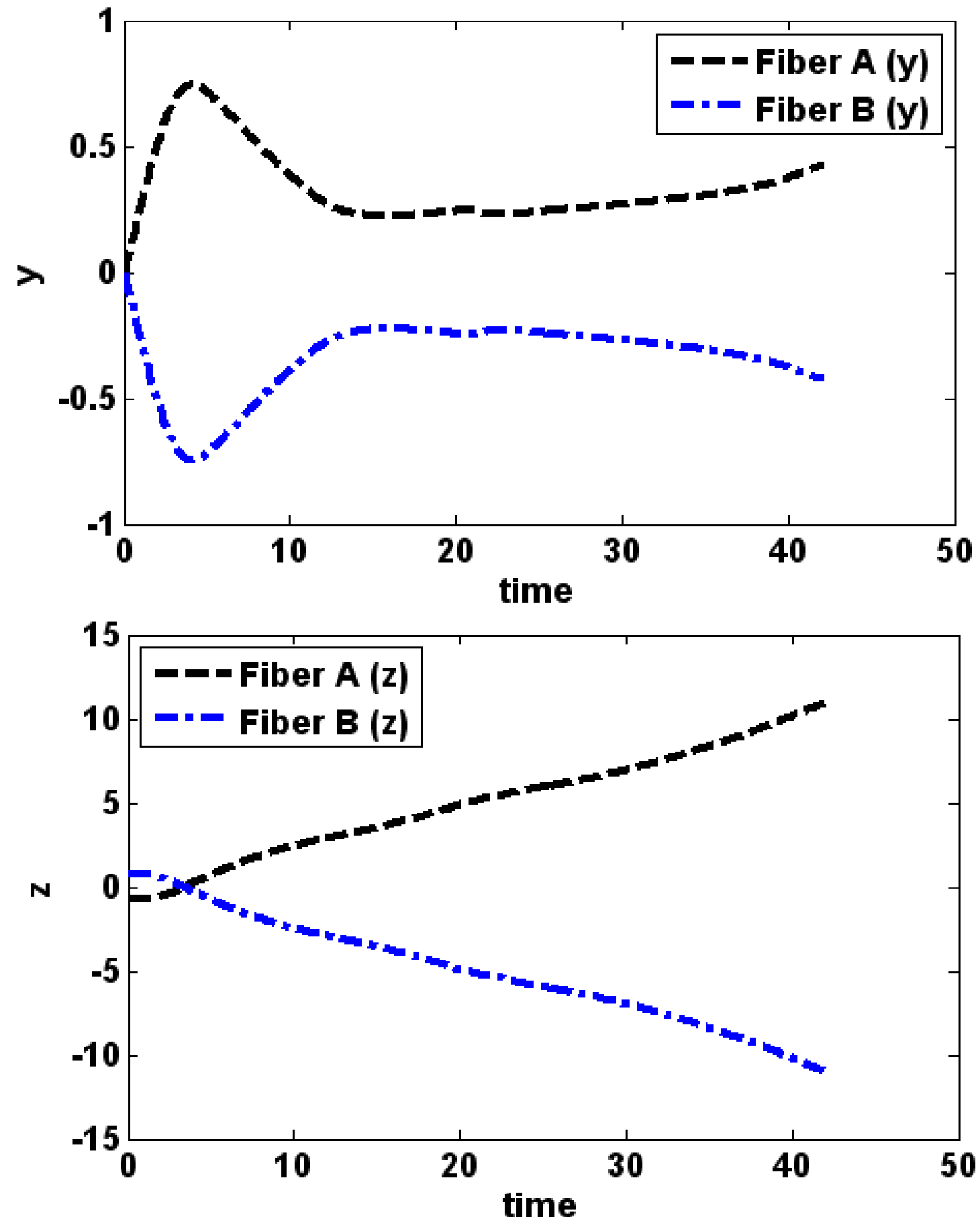

Figure 5.13: Fiber translations of two long ellipsoidal fibers $(\Im=0.25)$

The change of fibers' linear velocities are shown in Fig.5.13, from which it can be seen that the motions of two long fibers are different from those of two short fibers in Example 1. In this example, fibers interact with each other, and start to slide away 
with each other at the early stage of one period of fiber rotation, while two short fibers spend more time interacting with each other and start to slide away at the end of one complete period. Therefore, the aspect ratio of the fiber has a critical effect on the fiber motion.

\subsubsection{Effect of multiple fiber suspensions}

As seen in the previous section, fiber orientation is retarded with the existence of another fiber, but fiber still has a tumbing motion within the fluid. The hydrodynamic effect forces the fibers to rotate slowly. But in Advani and Tucker III's paper [12], fibers begin to align along the bulk flow, which is not observed in our simulations by considering the fiber-fiber interaction between two close fibers. Therefore, in this section, we investigate the effect of multiple fiber suspensions by simulating the fiber motion in a reduced fluid domain. As discussed in Sec.5.3, we use $\varepsilon$ to investigate the effect of the bounded fluid domain. We simulate the fiber motions in a bounded fluid domain with various sizes, and the dimensionless simulation parameters are set up in Table 5.3. The examples shown in this section are implemented using the three-dimensional FEM with the planar motion of fiber.

\begin{tabular}{|c|c|}
\hline Properties & Values \\
\hline \hline fiber size (ellipsoidal fiber) & $a=3, b=1, r_{e}=a / b=3$ \\
\hline size of fluid domain $(2 R)$ & $120,20,10,5,3.75$ or 3.34 \\
\hline ratio of fluid domain size to fiber size $(\varepsilon)$ & $40,6.67,3.33,1.67,1.25$ or 1.11 \\
\hline initial orientation & $\phi_{0}=0, \theta_{0}=\pi / 3, \psi_{0}=0$ \\
\hline initial position & $x_{c}=0, y_{c}=0, z_{c}=0$ \\
\hline properties of bulk fluid & $\mu=1, U_{z}=\dot{\gamma} y$, where $\dot{\gamma}=1$ \\
\hline
\end{tabular}

Table 5.3: Parameters to simulate fiber motion in a bounded fluid domain

Fiber orientations and time periods according to different $\varepsilon^{\prime} s$ are shown in Fig.5.14, from which we can see that when $\varepsilon \geq 40$, the true percent relative error of time period compared with that obtained from Jeffery's solution is below $0.08 \%$. The period of fiber rotation increases as $\varepsilon$ decreases, i.e., the dimension of fluid domain becomes 
closer to fiber size. More importantly, when $\varepsilon$ is close to 1.25 for this case, i.e. the size of fluid domain is 1.25 times larger than the fiber size, fiber orientation is stuck at certain angle. The angle $\phi$ keeps to be around $\pi / 2$ when $\varepsilon=1.25$, while $\phi$ remains $0.44 \pi$ when $\varepsilon=1.11$. This finding physically explains the effect of multiple fiber suspensions and the fiber alignment in Folgar-Tucker III's model [10].

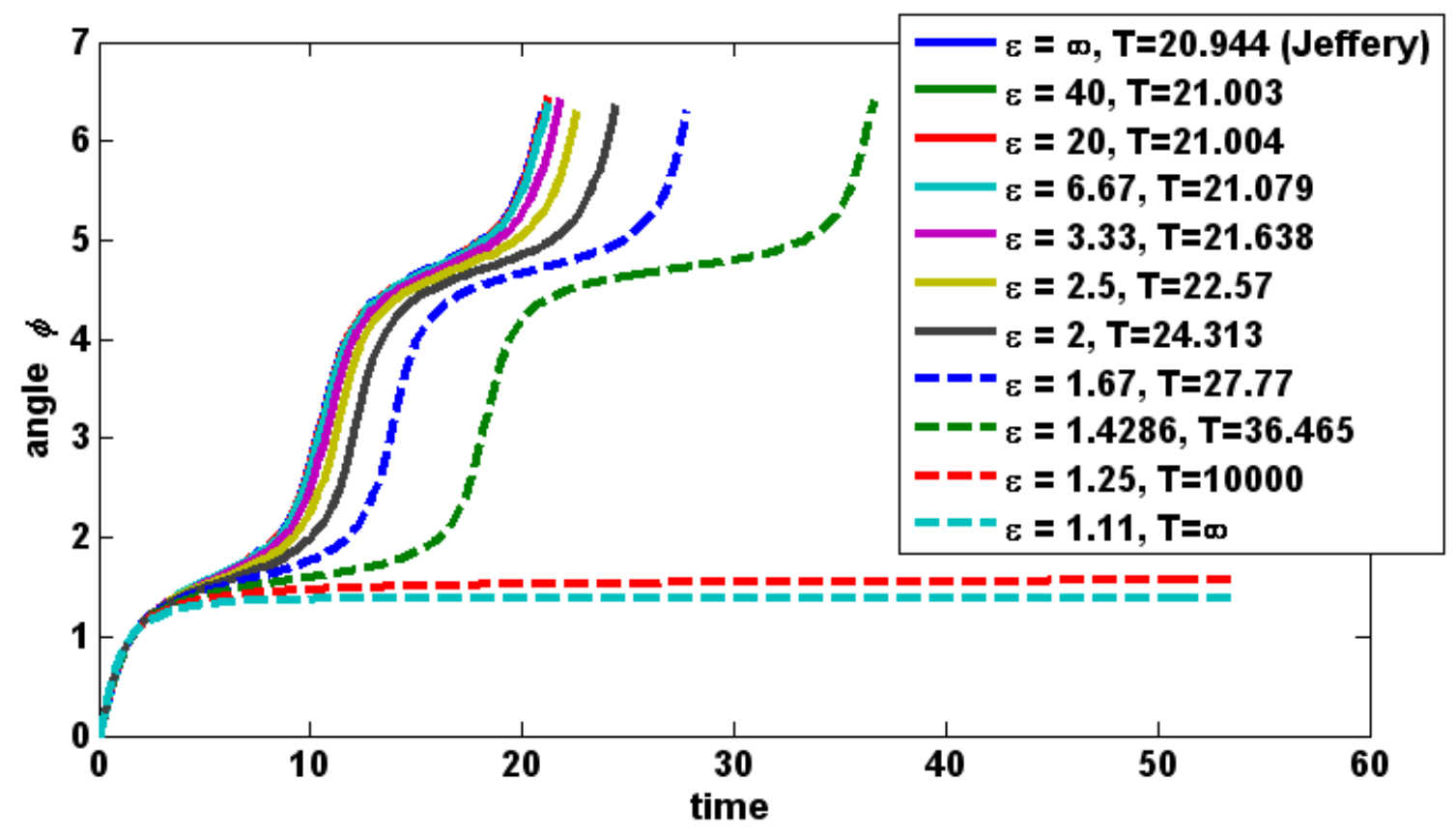

Figure 5.14: Effect of various $\varepsilon^{\prime} s$ on fiber orientations (evolution of $\phi$ ).

It has been discussed that fiber orientation results from the hydrodynamic torque in bulk fluid. In this section, we use the proposed FEM to analyze the torque that contributes to fiber orientation ( $\phi$ in this case). If the fiber is fixed with different orientations, the torques using different sizes of fluid domain are shown in Fig.5.15. For a large $\varepsilon$, the torque is always larger than zero, which pushes the fiber to rotate continuously, while when $\varepsilon<1.25$, the torque goes cross the zero line, meaning that fiber orientation is stuck at that point. When $\varepsilon=1.11$, the torque becomes to be zero at $\phi=0.44 \pi$, which explains the fiber alignment in Fig.5.14.

Therefore, the bounded fluid domain retards fiber orientation, and also results in fiber stagnation when $\varepsilon$ becomes very small. In this chapter, we proposed to use the 


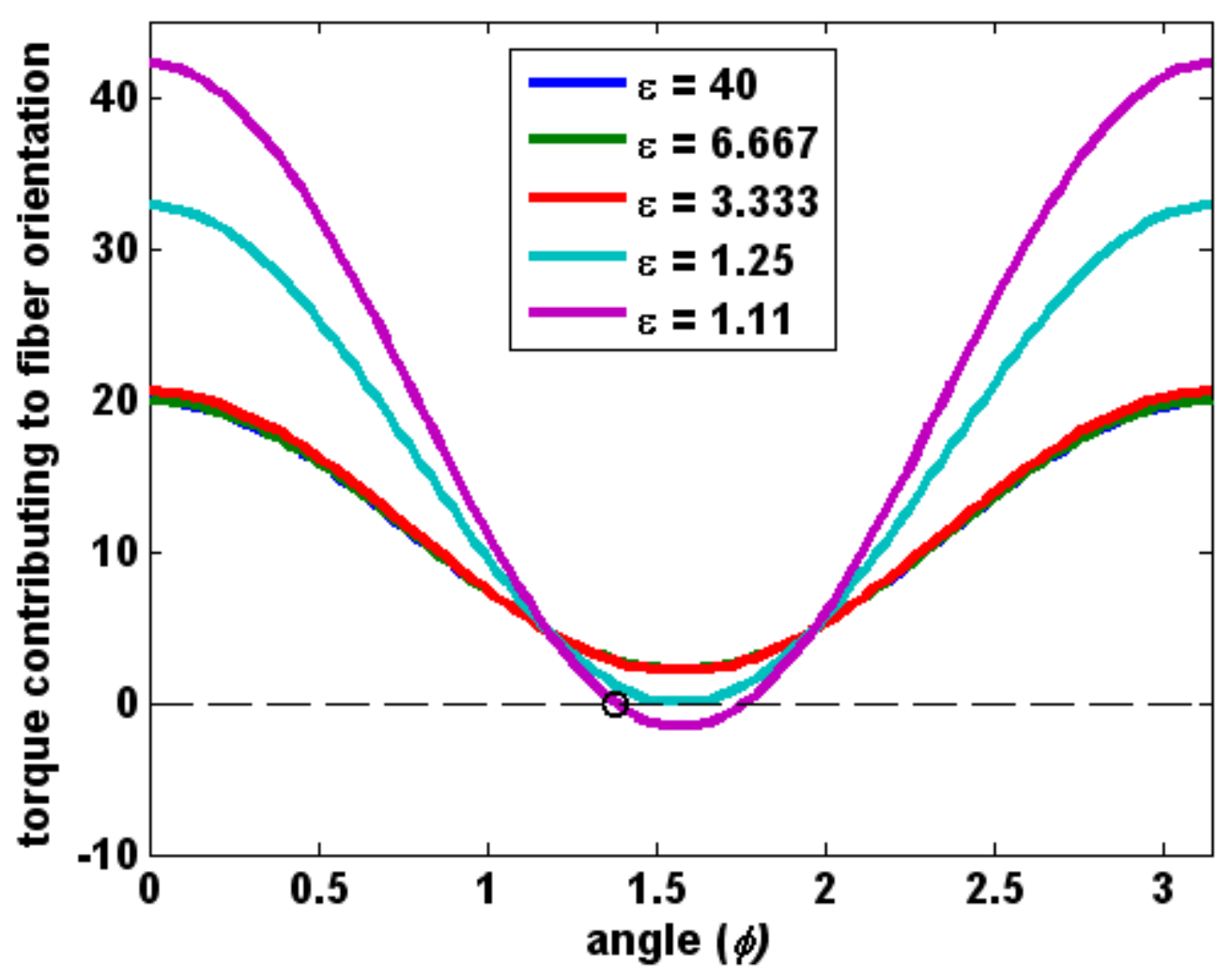

Figure 5.15: Plot of torques with different fiber orientation using various fluid domain.

equivalent aspect ratio, defined in Sec.5.3 to quantify the effect of the bounded fluid domain. With the given fluid domain, fiber's equivalent aspect ratios are tabulated in Table 5.4 using Eq.(5.10).

\begin{tabular}{|c|c|c|}
\hline Parameter $\varepsilon$ & Equivalent aspect ratio $\left(r_{e}^{*}\right)$ & Equivalent $\lambda$ \\
\hline \hline 40 & 3.01 & 0.8 \\
\hline 6.67 & 3.02 & 0.8 \\
\hline 3.33 & 3.12 & 0.81 \\
\hline 1.67 & 4.18 & 0.89 \\
\hline 1.25 & 43.4 & 0.99 \\
\hline 1.11 & $5.24 i$ & $1.08(>1)$ \\
\hline
\end{tabular}

Table 5.4: Equivalent aspect ratios of fibers in a bounded fluid domain with various sizes $\left(r_{e}=3\right)$

By comparing the results using the bounded fluid domain with Jeffery's unbounded model using the calculated equivalent aspect ratio, we find in Fig.5.16 that fiber motion in a bounded fluid domain is still governed by Jeffery's equation, but 

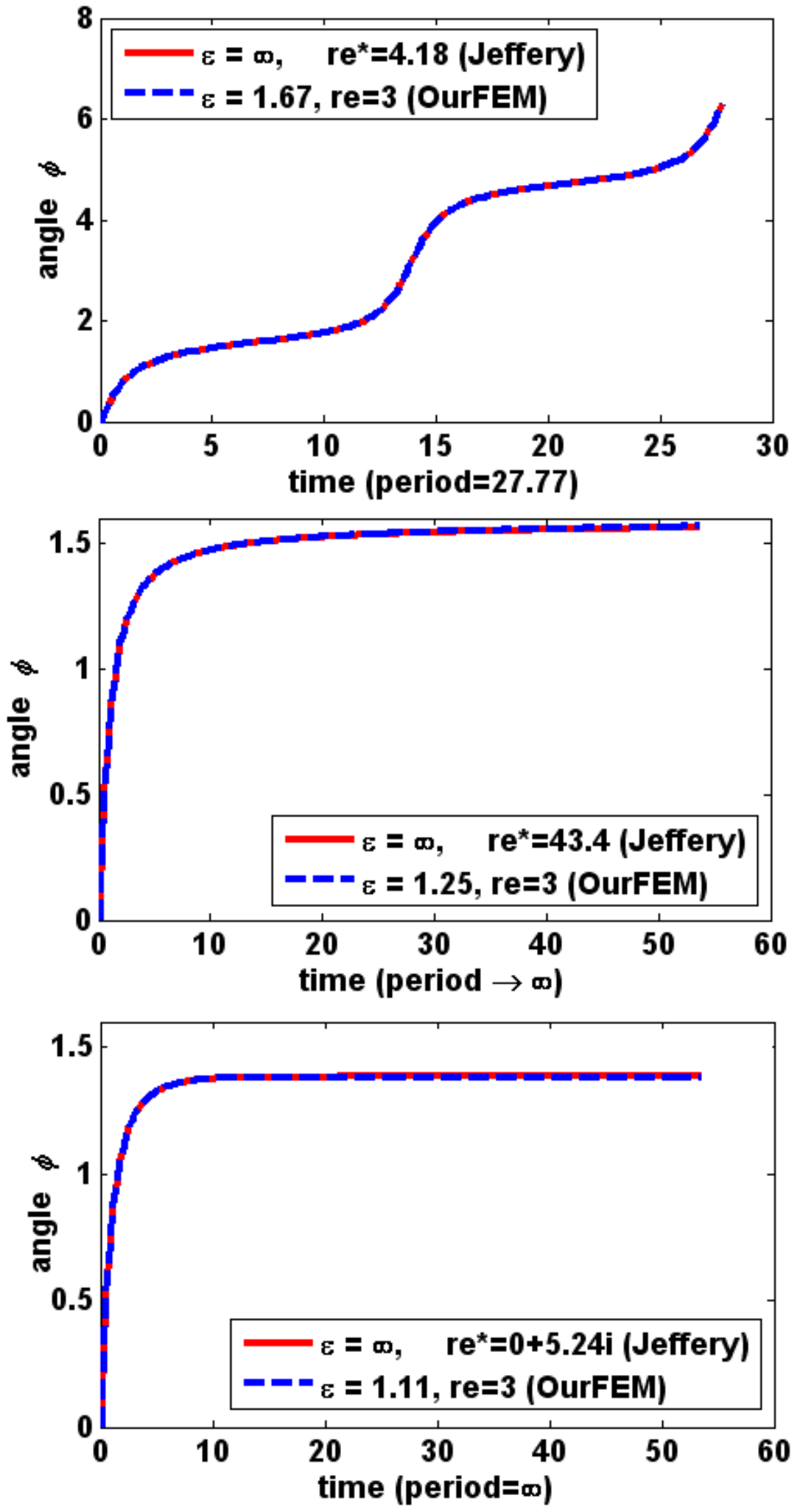

Figure 5.16: Fiber orientations in the bounded fluid domain. 
the equivalent aspect ratio is used to quantify the fiber motion. More importantly, when fluid domain is close to fiber size, the equivalent aspect ratio becomes a complex number and the fiber comes to an alignment. As seen in Fig.5.16, fiber motion in a bounded fluid domain obtained from the proposed finite element model is in excellent agreement with Jeffery's analytical solution using the calculated equivalent aspect ratio. In Fig.5.16(c), $\varepsilon=1.11$, fiber's equivalent aspect ratio becomes 5.24i, and the corresponding equivalent $\lambda$ becomes 1.08. For an ellipsoidal fiber, the relationship between the equivalent $\lambda$ and $\varepsilon$ is shown in Fig.5.17, from which it can be seen that as the fluid domain gets close to fiber, the rate of change of equivalent $\lambda$ gets faster. When the fluid domain gets farther away from the fiber, the equivalent aspect ratio of fiber converges to fiber's geometric aspect ratio for an ellipsoidal fiber.

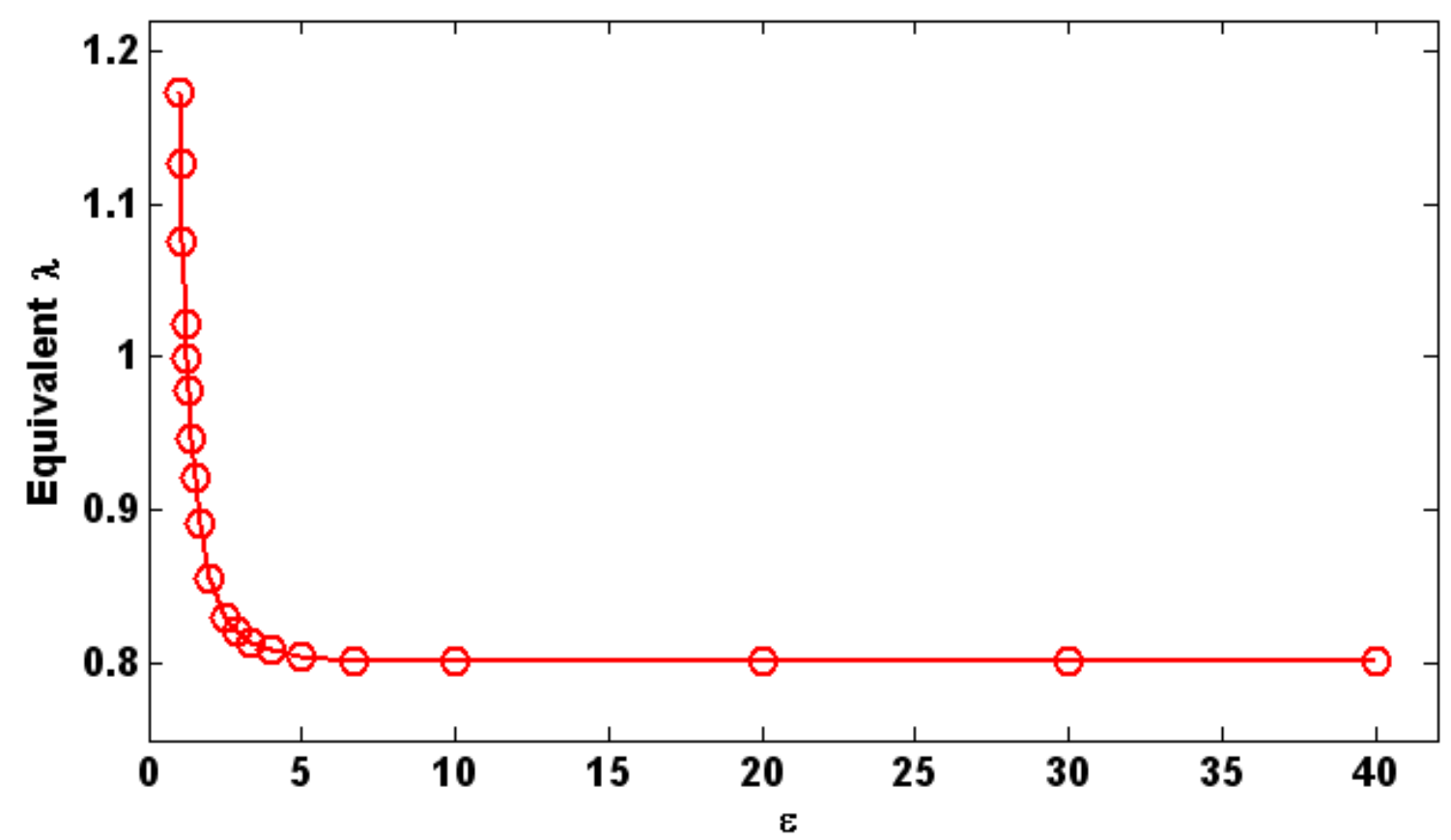

Figure 5.17: Relationship between equivalent $\lambda$ and $\varepsilon$ for an ellipsoidal fiber with $r_{e}=3$.

The same phenomenon is seen for any other symmetric fibers, such as cylindrical fibers. In this chapter, we proposed that equivalent aspect ratio is a function of both fiber shape and the parameter $\varepsilon$, which describes the ratio of fluid domain size to 
fiber size. We use a cylindrical fiber with the geometric aspect ratio 10 to investigate the effect of fluid domain. The relationship between the equivalent $\lambda$ and $\varepsilon$ is shown in Fig.5.18, from which we can see that the cylindrical fiber's equivalent aspect ratio converges to 7.5 with a large fluid domain.

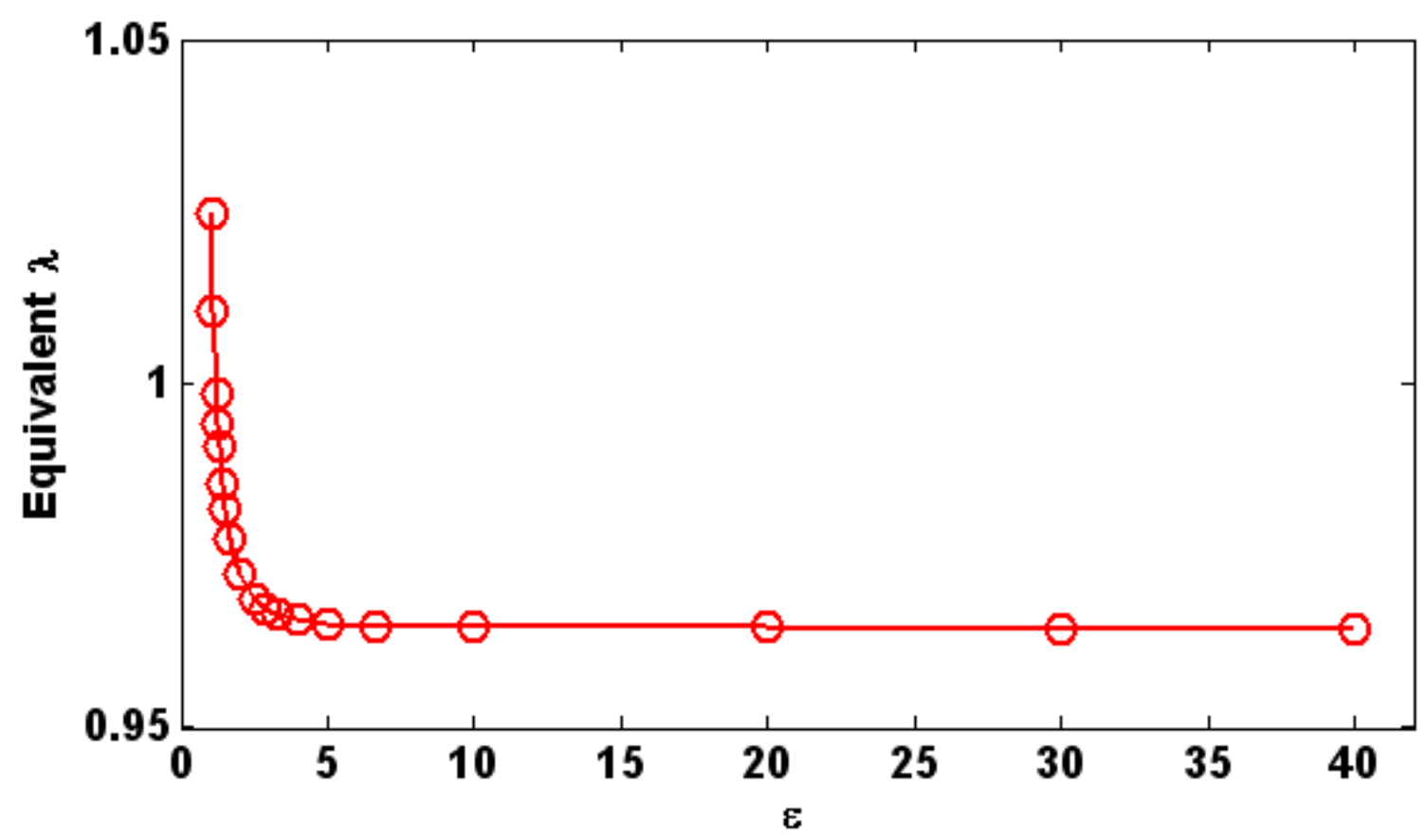

Figure 5.18: Relationship between equivalent $\lambda$ and $\varepsilon$ for a cylindrical fiber with $r_{e}=10$.

\subsubsection{Fiber motion near a stationary wall}

In a mold cavity during short-fiber reinforced composites processing, the motions of fibers close to one side of the mold wall are different from those far away from the wall. In this section, we investigate the fiber motion near a stationary wall using the proposed methodology. We use the distance $\Delta d$ between the fiber centroid and the stationary wall to quantify the effect of wall, defined in Fig.5.19.

Fiber orientation and position are shown in Fig.5.20, from which it can be seen that fiber orientation deviates from Jeffery's prediction and is retarded with the existence 


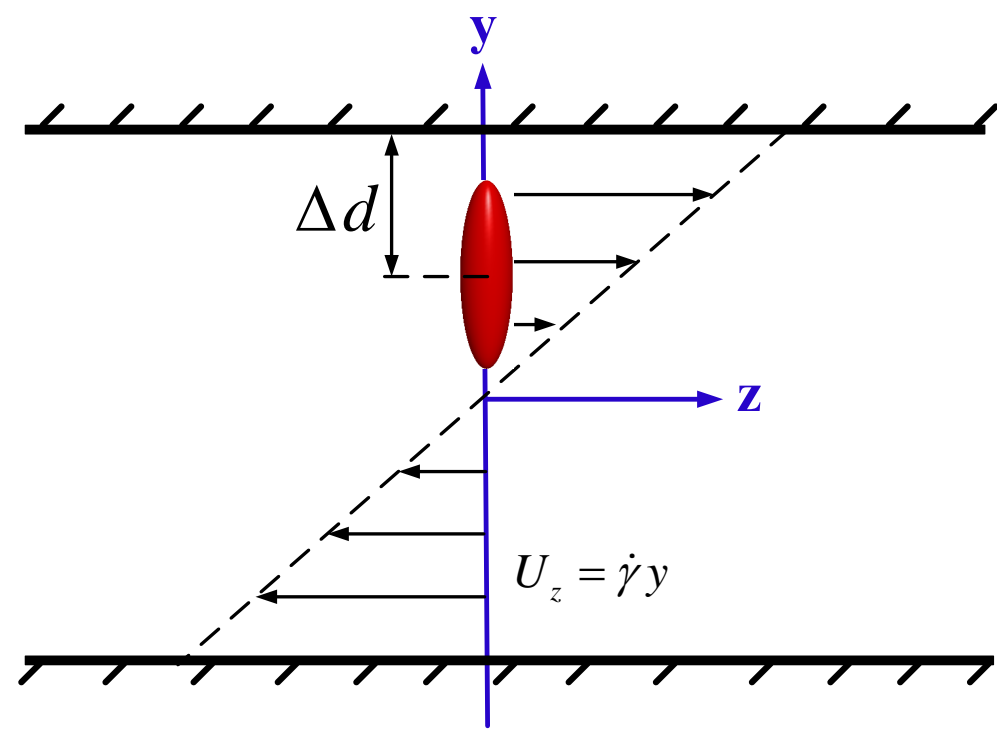

Figure 5.19: Definition of the effect of a stationary wall.

of a stationary wall. Meanwhile, fiber has the up-and-down motion towards the wall ( $y$ direction), which is not observed for the motion of fiber far away from the wall.

We implement the simulations using a wide range of $\Delta d^{\prime} s$, and investigate the effect of a stationary wall on fiber motion. The time period of fiber rotation with respect to $\Delta d$ is plotted in Fig.5.21, from which it can be seen that as fiber gets closer to the wall, fiber rotation gets slower.

\subsection{Summary}

The FEM-based methodology proposed in Chapter 3 is extended to solve the motions of multiple fibers suspended within a viscous fluid. The hydrodynamic interaction between two fibers, the effect of bounded fluid domain and fiber motion near a stationary wall are studied. Three issues are related to the disturbance effect of the presence of other objects (fibers or a bounded wall) on fiber motion. We find that the hydrodynamic interaction only takes effect when two fibers are close to each other, which would slow down fiber motions. The existence of multiple fibers around a fiber is modeled as the bounded fluid domain around the fiber. We observe that fiber starts 

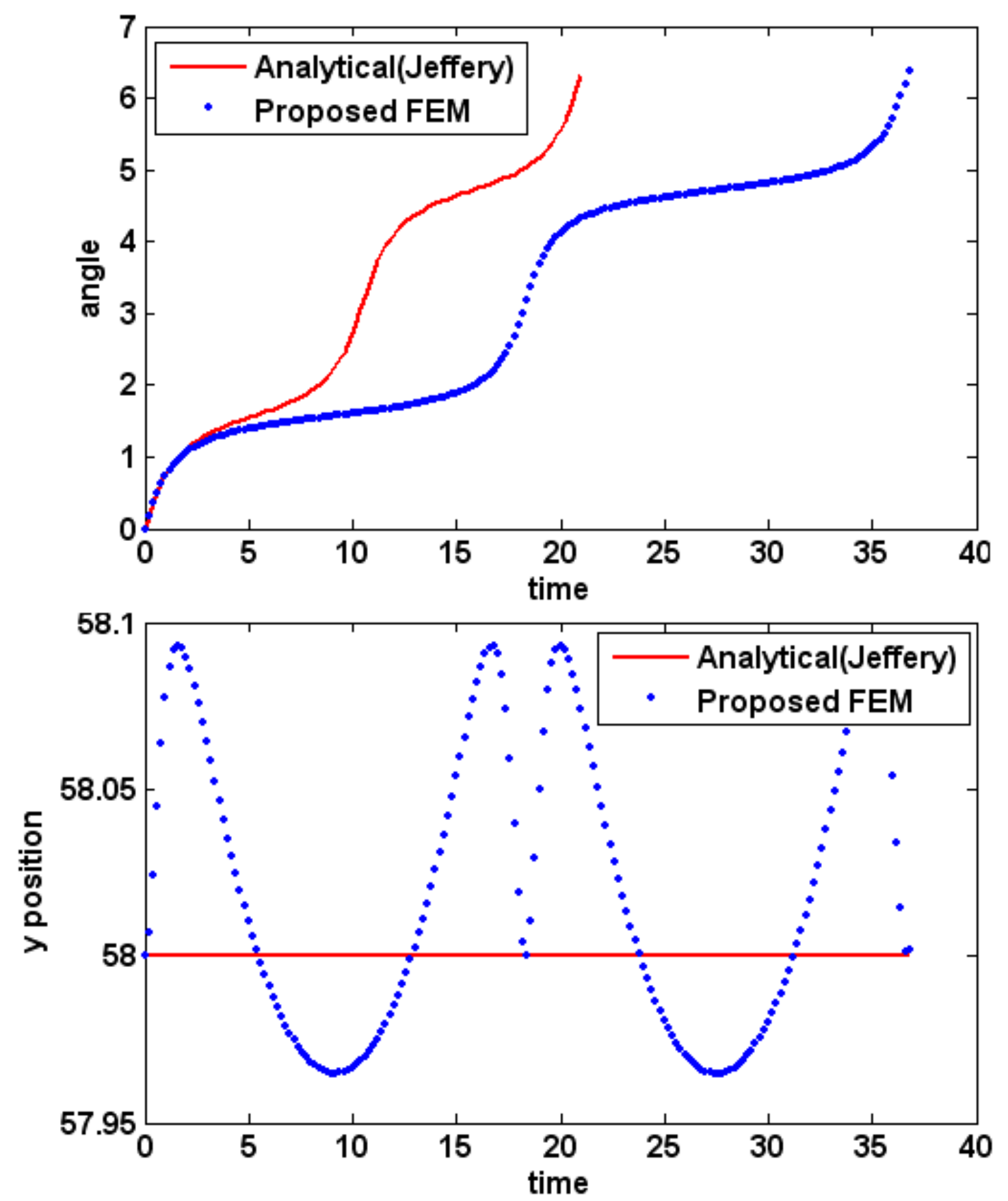

Figure 5.20: Fiber motion near a stationary wall: (top) fiber orientation and (bottom) fiber position in the y direction

to align with the bulk flow when the fluid domain is very close to fiber, which explains the effect of $D_{r}$ term in Folgar-Tucker III's model [10]. Also, we investigate the fiber motion near a stationary wall, and find that the fiber motion is regarded with the existence of a wall. Fiber travels with the bulk fluid, and performs the up-and-down 


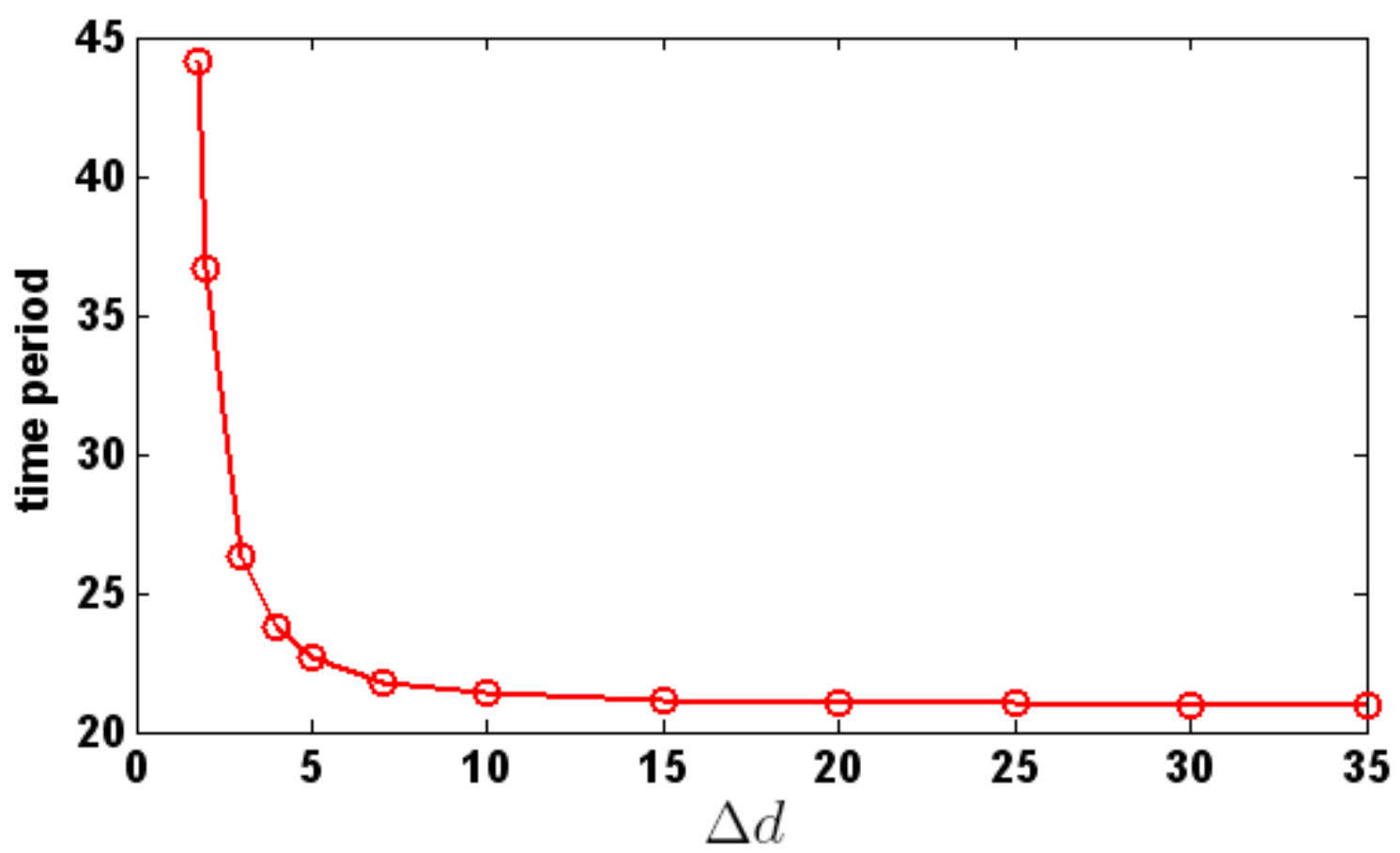

Figure 5.21: Relationship between time period of fiber rotation and parameter $\Delta d$ motion. 


\section{Chapter 6}

\section{Summary and Concluding Remarks}

\subsection{Summary of the dissertation}

This research work focuses on gaining a fundamental understanding of micro- and nano-fiber suspensions during the manufacture of polymer composites through the numerical simulation. The successful completion of this project provides a systematic computational approach capable of addressing issues that are currently unresolved in the critical area of composite manufacturing, in which mechanical, electrical and thermal properties of short-fiber reinforced composite materials are largely dependent on fiber orientations within the polymer matrix during the manufacturing process. This research work uses a unique approach to evaluate fiber orientations within the fluid, providing a means for controlling the fiber orientations in the products.

In this research work, we proposed a FEM-based simulation tool, which combines the Finite Element Method, a Newton-Raphson iteration and a Runge-Kutta method, to solve the general three-dimensional motion of a single suspended fiber within a viscous fluid [85]. This method is very general and can be applied to any axisymmetric fiber, and in the present study results are presented for ellipsoidal, cylindrical and 
bead-chain fibers. We demonstrate that fiber shape has a significant impact on the fiber orientation, which affects the rate of fiber alignment in short-fiber reinforced composite materials. For an axisymmetric fiber, the equivalent aspect ratio is needed for numerical solutions of the Jeffery's equation for the fiber motion instead of the geometric aspect ratio. The equivalent aspect ratios of cylindrical fibers and other fiber types are generated using the proposed numerical method, and are validated by evaluating the fiber motion numerically. In Chapter 2, the commercial FEM solver is used, so we have no access to the stiffness matrix, which costs huge computation time.

In order to solve fiber motions in complex flow conditions efficiently, we proposed a stand-alone FEM-based package coupled with analytical Jacobian matrix from the finite element model and transferred essential boundary condition in Chapter 3. The proposed simulation approach achieves forty times better time efficiency than that using the commercial FEM solver. Fiber motions in various Poiseuille flows are investigated, and compared with existing theories and experimental findings. Our results confirm to Bretherton's conclusion that the Segré-Silberberg phenomenon does not exist in unbounded creeping Poiseuille flows. In a Poiseuille flow, the fiber performs a tumbling motion, which is governed by Jeffery's equation using the flow shear rate evaluated at fiber centroid. Another interesting finding is that the fiber has a change of linear velocity in the direction of the flow field. This is caused by the quadratic flow component, with the hydrodynamic force dragging the fiber backwards. As the fiber rotates, the driving force changes accordingly.

As nano-fiber reinforced composite materials become popular, we proposed a FEM-based Brownian dynamics simulation tool for modeling the motion of nanofibers during the nano-polymer composites processing [88]. A stand-alone Finite Element Method is developed to study the hydrodynamic effect and Brownian dynamics simulation is used to account for the Brownian effect. From our simulation 
results, we find that when fiber size decreases, Brownian effect comes into play and disturbs the fiber motion. The dimensionless Péclet number is calculated to quantify the Brownian effect. At a high Péclet number, fiber motion is still governed by Jeffery's theory, since the Brownian effect is insignificant. At a medium Péclet number, both hydrodynamic and Brownian effects come into play, and govern the fiber motion. At a small Péclet number, Brownian effect becomes very strong compared with hydrodynamic effect, so fiber motion is tremendously disturbed by the fluid molecules. In this dissertation, two-dimensional FEM-based Monte-Carlo simulation is used to predict the planar motions of nano-fibers.

In short-fiber reinforced composites, fiber-fiber interactions have a great effect on fiber motions. The proposed FEM-based simulation tool is extended to solve the motion of multiple suspended fibers within a viscous fluid. The hydrodynamic interaction between two fibers, the effect of bounded fluid domain and fiber motion near a stationary wall are studied [89]. We find that the hydrodynamic interaction only takes effect when two fibers are close to each other, which would slow down the fiber motion. The existence of multiple fibers around a fiber is modeled as the bounded fluid domain around the fiber. We observe that fiber starts to align with the bulk flow when the fluid domain is very close to fiber, which explains the effect of $D_{r}$ term in Folgar and Tucker III's model [10]. Also, we investigate the fiber motion near a stationary wall, and find that the fiber motion is retarded with the existence of a wall. Fiber travels with the bulk fluid, and performs the up-and-down motion.

\subsection{Thesis contributions}

The major contributions of this dissertation are summarized as follows:

1. A FEM-based simulation tool is developed to investigate the general threedimensional motion of discrete suspended fibers within the polymeric melt during the 
mold filling process. This method is very general and can be applied to the motion of any axisymmetric fiber in various flow situations. Under the same conditions, our numerical results are in excellent agreement with Jeffery's theoretical derivation. To our best knowledge, this is the first time that Jeffery's theory is validated using a three dimensional FEM-based approach.

2. The role of fiber shape is investigated in this research work. The equivalent aspect ratios of cylindrical fibers, bead-chain fibers and any axisymmetric fibers are derived to quantify fiber motions. For the industry-used cylindrical fibers, an empirical equation is proposed for both short and long rod-like fibers. For the bead-chain fibers, which are wildly used to model flexible fibers, different equivalent aspect ratios are obtained with and without the flow shielding. This research work benefits the modeling of flexible fibers using a chain of beads.

3. Fiber motions in Poiseuille flows are investigated. For the mold filling process, it is inappropriate to describe the polymeric melt as a homogeneous flow, so the direct implementation of Jeffery's theory in the mold filling process is unacceptable. The study of inhomogeneous flow suspensions completed in this research work provides an accurate prediction of fiber motion during the mold filling process.

4. Considering the excellent material properties of nano-fiber reinforced polymers, this research work investigates the Brownian motions of nano-fibers within the polymeric melt. The rheological properties of nano-fiber reinforced polymers are further complicated by the reduced size of the inclusions in the polymeric melt. Therefore, built on our research work on micro-fiber suspensions, we investigate the motions of nano-fibers in nano-fiber reinforced polymers.

5. Physical modeling of fiber-fiber interactions is implemented in this research work to gain a fundamental understanding of fiber behavior with the existence of multiple fibers. The current modification of Jeffery's equation to take into account fiber-fiber interactions is based on the empirical data gained from experiments, but 
the physical understanding of fiber-fiber interactions is still in question. This research work using two discrete fibers to model the fiber-fiber interaction offers current research community a new insight into the prediction of multiple fiber motions. Furthermore, the existence of multiple fibers around a fiber is modeled as the fiber motion in a bounded fluid domain to investigate the semi-dilute fiber suspensions.

\subsection{Future work}

The current research work in this dissertation can be extended in the future to the following aspects:

1. Concentrated fiber suspensions with mechanical contacts between fibers: When the fiber concentration is higher or the flow is nonlinear [90], non-hydrodynamic interactions such as physical contacts come into play. Mechanical contacts between fibers in concentrated suspensions could give rise to many nonlinear rheological characteristics such as finite normal stress differences [91], yield stress [92] and shear thinning. This research work can be extended to study concentrated fiber suspensions, i.e., the volume fraction of fibers is so high that physical/mechanical contacts dominate. The contact detection algorithms $[93,94]$ need to be added to the proposed simulation tool.

2. The motions of long flexible fibers: For the fiber with a high geometric aspect ratio, the rigid-body assumption is not appropriate, and fiber will bend and twist within the polymer matrix during the manufacturing process. Therefore, the investigation of fiber's bending and torsion is critical for long fiber suspensions. The most popular simulation method is to use a couple of beads or rods to model the flexible fibers, as seen in Refs.[26, 27, 95-98]. But these methods involve the tedious calculation of connecting forces between adjacent beads or rods. Meanwhile, the summation

of hydrodynamic force on each separate bead or rod does not really reflect the total 
force on the whole fiber because of the flow shielding by other fibers, as discussed in Chapter 2. This research work can be extended to investigate the bending and torsion of long fibers within the resin by adding the structure analysis of fiber into current simulation tool.

3. Fiber motions in non-Newtonian flows: In composites processing, most thermoplastic resins exhibit non-Newtonian behaviors [99-101]. Ganani [102] found that a polyisobutylene in cetane solution behaves as a second-order fluid at low shear-rates and shear-thinned at higher values. Chan [103] measured the shear viscosity and elongation viscosity for polyethylene and polystyrene melts. Phan-Thien et al. [104] extended the double layer boundary integral equation to viscoelastic fluid and solved the mobility problem for a system of particles in the Odroyd-B fluid. Goddard [105] studied the stress contribution from particle suspensions in non-Newtonian fluids. Leal [20] studied the particle suspension in a slightly non-Newtonian fluid by considering the non-Newtonian contribution to the total stress. The proposed FEM-based simulation tool can be extended to study fiber suspensions in inelastic flows, such as a power-law flow. The difference for inelastic flow is that fluid viscosity depends on the local velocity gradient. So the updating process of local fluid viscosity can be added into our current simulation tool to investigate fiber suspensions in inelastic flows.

Extension of the proposed approach to other areas such as drug delivery, blood cell motion, and air dust drift in environment protection will be an additional benefit of the proposed research. The successful completion of this research work would lead to a better understanding of rheological properties in blood. In this research work, the proposed stand-alone FEM-based simulation tool competes current commercial finite element software, such as COMSOL Multiphysics. Thus there is a great opportunity to commercialize the proposed finite element package for the prediction of fiber orientation in composite manufacturing process. The commercialization of this package has a widely application in composite manufacturing industry to enhance the 
precision of fiber prediction in composites during the manufacturing process. 


\section{Appendix A}

\section{Three-dimensional Finite Element Method in Solving Fiber Motion within a Viscous Flow}

\section{A.1 Basis of fluid mechanics}

\section{A.1.1 Governing equations}

The governing equations for isothermal incompressible Stokes fluid are Continuity equation:

$$
-\frac{\partial \rho}{\partial t}+\nabla \cdot(\rho \mathbf{U})=0
$$

Momentum equation:

$$
-\nabla \cdot \sigma+\rho \mathbf{f}=\mathbf{0}
$$

where

$$
\boldsymbol{\sigma}=-p \mathbf{I}+2 \mu \Gamma
$$


In Eq.(A.3), $\sigma$ can be expanded using velocity vector $\mathbf{U}=[u, v, w]^{T}$ and pressure $p$, shown as

$$
\sigma=\left[\begin{array}{ccc}
-p+2 \mu \frac{\partial u}{\partial x} & \mu\left(\frac{\partial v}{\partial x}+\frac{\partial u}{\partial y}\right) & \mu\left(\frac{\partial w}{\partial x}+\frac{\partial u}{\partial z}\right) \\
\mu\left(\frac{\partial v}{\partial x}+\frac{\partial u}{\partial y}\right) & -p+2 \mu \frac{\partial v}{\partial y} & \mu\left(\frac{\partial w}{\partial y}+\frac{\partial v}{\partial z}\right) \\
\mu\left(\frac{\partial w}{\partial x}+\frac{\partial u}{\partial z}\right) & \mu\left(\frac{\partial w}{\partial y}+\frac{\partial v}{\partial z}\right) & -p+2 \mu \frac{\partial w}{\partial z}
\end{array}\right]
$$

\section{A.1.2 Boundary conditions}

There are two kinds boundary conditions in solving governing equations in fluid.

Dirichlet/essential boundary conditions (velocity/pressure):

$$
\mathbf{U}_{i}=\mathbf{U}_{\text {known }}
$$

Neumann/natural boundary conditions (total stress):

$$
\mathbf{T}_{i}=\sigma \cdot \mathbf{n}=\mathbf{T}_{\text {known }}
$$

Equation (A.6) can be expanded as,

$$
\mathbf{T}_{i}=\left[\begin{array}{c}
\left(-p+2 \mu \frac{\partial u}{\partial x}\right) n_{1}+\mu\left(\frac{\partial v}{\partial x}+\frac{\partial u}{\partial y}\right) n_{2}+\mu\left(\frac{\partial w}{\partial x}+\frac{\partial u}{\partial z}\right) n_{3} \\
\mu\left(\frac{\partial v}{\partial x}+\frac{\partial u}{\partial y}\right) n_{1}+\left(-p+2 \mu \frac{\partial v}{\partial y}\right) n_{2}+\mu\left(\frac{\partial w}{\partial y}+\frac{\partial v}{\partial z}\right) n_{3} \\
\mu\left(\frac{\partial w}{\partial x}+\frac{\partial u}{\partial z}\right) n_{1}+\mu\left(\frac{\partial w}{\partial y}+\frac{\partial u}{\partial z}\right) n_{2}+\left(-p+2 \mu \frac{\partial w}{\partial z}\right) n_{3}
\end{array}\right]
$$

where $\mathbf{n}=\left[n_{1}, n_{2}, n_{3}\right]^{T}$ is the unit normal direction of the nodes on the boundary.

\section{A.2 Finite Element Method for Newtonian fluids}

In this dissertation, we use the mixed finite element model to solve fluid mechanics problem [64]. Mixed model means that both velocity and pressure appear in the weak 
form, so velocity and pressure are solved simultaneously in the finite element model.

\section{A.2.1 Weak form of governing equations}

For isothermal incompressible low-Reynolds-number fluids, the governing equations are simplified shown as

$$
\begin{aligned}
\nabla \cdot \mathbf{U} & =0 \\
-\nabla \cdot \sigma & =0
\end{aligned}
$$

The governing equations in the strong form are converted into the weak form using the weight/shape function ( $\Psi$ for velocity and $\Phi$ for pressure) and integrationby-parts, with the continuity equation and momentum equation shown as

$$
\begin{aligned}
& \int_{\Omega} \Phi\left[\frac{\partial u}{\partial x}+\frac{\partial v}{\partial y}+\frac{\partial w}{\partial z}\right] d x d y d z=0 \\
& \int_{\Omega} \frac{\partial \Psi}{\partial x}\left[-p+2 \mu \frac{\partial u}{\partial x}\right] d x d y d z+\int_{\Omega} \frac{\partial \Psi}{\partial y}\left[\mu\left(\frac{\partial v}{\partial x}+\frac{\partial u}{\partial y}\right)\right] d x d y d z \\
& \quad+\int_{\Omega} \frac{\partial \Psi}{\partial z}\left[\mu\left(\frac{\partial w}{\partial x}+\frac{\partial u}{\partial z}\right)\right] d x d y d z-\oint\left[\Psi\left(-p+2 \mu \frac{\partial u}{\partial x}\right)\right] n_{1} d S \\
& \quad-\oint\left[\Psi \mu\left(\frac{\partial v}{\partial x}+\frac{\partial u}{\partial y}\right)\right] n_{2} d S-\oint\left[\Psi \mu\left(\frac{\partial w}{\partial y}+\frac{\partial v}{\partial z}\right)\right] n_{3} d S=0 \\
& \int_{\Omega} \frac{\partial \Psi}{\partial x}\left[\mu\left(\frac{\partial v}{\partial x}+\frac{\partial u}{\partial y}\right)\right] d x d y d z+\int_{\Omega} \frac{\partial \Psi}{\partial y}\left[-p+2 \mu \frac{\partial v}{\partial y}\right] d x d y d z \\
& \quad+\int_{\Omega} \frac{\partial \Psi}{\partial z}\left[\mu\left(\frac{\partial w}{\partial y x}+\frac{\partial v}{\partial z}\right)\right] d x d y d z-\oint\left[\Psi \mu\left(\frac{\partial v}{\partial x}+\frac{\partial u}{\partial y}\right)\right] n_{1} d S \\
& \quad \oint_{[}\left[\Psi\left(-p+2 \mu \frac{\partial v}{\partial y}\right)\right] n_{2} d S-\oint\left[\Psi \mu\left(\frac{\partial w}{\partial y}+\frac{\partial v}{\partial z}\right)\right] n_{3} d S=0 \\
& \int_{\Omega} \frac{\partial \Psi}{\partial x}\left[\mu\left(\frac{\partial w}{\partial x}+\frac{\partial u}{\partial z}\right)\right] d x d y d z+\int_{\Omega} \frac{\partial \Psi}{\partial y}\left[\mu\left(\frac{\partial w}{\partial y}+\frac{\partial v}{\partial z}\right)\right] d x d y d z \\
& \quad+\int_{\Omega} \frac{\partial \Psi}{\partial z}\left[-p+2 \mu \frac{\partial w}{\partial z}\right] d x d y d z-\oint\left[\Psi \mu\left(\frac{\partial w}{\partial x}+\frac{\partial u}{\partial z}\right)\right] n_{1} d S \\
& \quad \oint_{[}\left[\Psi \mu\left(\frac{\partial w}{\partial y}+\frac{\partial v}{\partial z}\right)\right] n_{2} d S-\oint\left[\Psi\left(-p+2 \mu \frac{\partial w}{\partial z}\right)\right] n_{3} d S=0
\end{aligned}
$$


where $\Omega$ is the domain of integration and $d S$ represents the surface integral on the domain $\Omega$.

\section{A.2.2 Discretization}

The independent variables are fluid velocity $\mathbf{U}=[u, v, w]^{T}$ and pressure $p$, and the shape functions are governed by $L B B$ compatibility condition [65], i.e. the weight function for pressure should be at least one order lower than that used for velocity field. For the consideration of simplicity, the Gauss quadrature is used to do the integral in the natural coordinate system $(\xi, \eta, \zeta)$. Therefore, the following expressions are all established in the local natural coordinate system.

$$
\begin{aligned}
& u(\xi, \eta, \zeta)=\Psi^{T}(\xi, \eta, \zeta)\{u\}=\{u\}^{T} \Psi(\xi, \eta, \zeta) \\
& v(\xi, \eta, \zeta)=\Psi^{T}(\xi, \eta, \zeta)\{v\}=\{v\}^{T} \Psi(\xi, \eta, \zeta) \\
& w(\xi, \eta, \zeta)=\Psi^{T}(\xi, \eta, \zeta)\{w\}=\{w\}^{T} \Psi(\xi, \eta, \zeta) \\
& p(\xi, \eta, \zeta)=\Phi^{T}(\xi, \eta, \zeta)\{p\}=\{p\}^{T} \Phi(\xi, \eta, \zeta)
\end{aligned}
$$

where $\Psi$ and $\Phi$ are the shape functions for velocity and pressure, respectively. In this case, velocity has the second-order shape function and pressure has the first-order shape function, with the element type shown in Fig.A.1. 

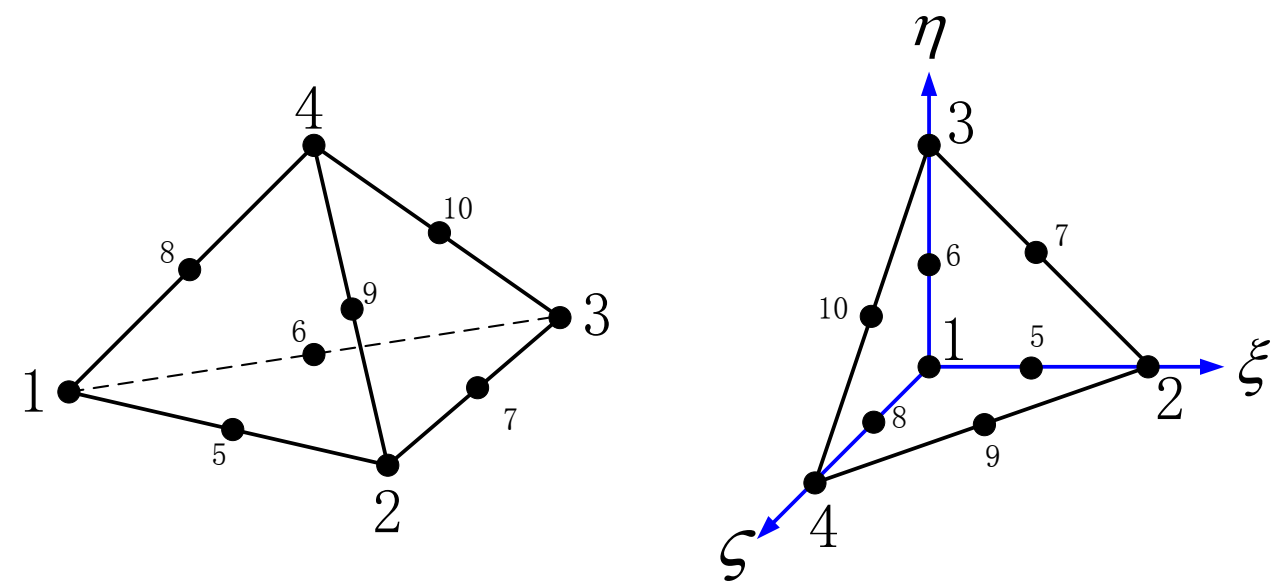

Figure A.1: Tetrahedral element: 10 nodes for velocity (nodes 1-10) and 4 nodes for pressure (nodes 1-4)

For velocity:

$$
\Psi=\left\{\begin{array}{c}
L_{1}\left(2 L_{1}-1\right) \\
L_{2}\left(2 L_{2}-1\right) \\
L_{3}\left(2 L_{3}-1\right) \\
L_{4}\left(2 L_{4}-1\right) \\
4 L_{1} L_{2} \\
4 L_{1} L_{3} \\
4 L_{2} L_{3} \\
4 L_{1} L_{4} \\
4 L_{2} L_{4} \\
4 L_{3} L_{4}
\end{array}\right\}
$$

For pressure:

$$
\Phi=\left\{\begin{array}{c}
L_{1} \\
L_{2} \\
L_{3} \\
L_{4}
\end{array}\right\}
$$

where $L_{1}=1-\xi-\eta-\zeta, L_{2}=\xi, L_{3}=\eta$, and $L_{4}=\zeta$. Note that the above shape functions are defined in the local natural coordinates $(\xi, \eta, \zeta)$. Therefore, the global 
coordinates $(x, y, z)$ are also interpolated using the isoparametric formulation, shown as

$$
\begin{aligned}
& x(\xi, \eta, \zeta)=\Psi(\xi, \eta, \zeta)^{T}\{x\}=\{x\}^{T} \Psi(\xi, \eta, \zeta) \\
& y(\xi, \eta, \zeta)=\Psi(\xi, \eta, \zeta)^{T}\{y\}=\{y\}^{T} \Psi(\xi, \eta, \zeta) \\
& z(\xi, \eta, \zeta)=\Psi(\xi, \eta, \zeta)^{T}\{z\}=\{z\}^{T} \Psi(\xi, \eta, \zeta)
\end{aligned}
$$

\section{A.2.3 System of equations}

The algebraic equation in the three-dimensional case is

$\left[\begin{array}{cccc}2 K_{11}+K_{22}+K_{33} & K_{21} & K_{31} & -Q_{1} \\ K_{12} & K_{11}+2 K_{22}+K_{33} & K_{32} & -Q_{2} \\ K_{13} & K_{23} & K_{11}+K_{22}+2 K_{33} & -Q_{3} \\ -Q_{1}^{T} & -Q_{2}^{T} & -Q_{3}^{T} & 0\end{array}\right]\left[\begin{array}{c}\{u\} \\ \{v\} \\ \{w\} \\ \{p\}\end{array}\right]=\left[\begin{array}{c}F_{1} \\ F_{2} \\ F_{3} \\ 0\end{array}\right]$

(A.21) 
where

$$
\begin{aligned}
& K_{11}=\int_{\Omega} \mu \Psi_{x} \Psi_{x}^{T} d x d y d z, \quad K_{22}=\int_{\Omega} \mu \Psi_{y} \Psi_{y}^{T} d x d y d z \\
& K_{21}=\int_{\Omega} \mu \Psi_{y} \Psi_{x}^{T} d x d y d z, \quad K_{12}=\int_{\Omega} \mu \Psi_{x} \Psi_{y}^{T} d x d y d z \\
& K_{31}=\int_{\Omega} \mu \Psi_{z} \Psi_{x}^{T} d x d y d z, \quad K_{13}=\int_{\Omega} \mu \Psi_{x} \Psi_{z}^{T} d x d y d z \\
& K_{23}=\int_{\Omega} \mu \Psi_{y} \Psi_{z}^{T} d x d y d z, \quad K_{32}=\int_{\Omega} \mu \Psi_{z} \Psi_{y}^{T} d x d y d z \quad Q_{3}=\int_{\Omega} \Psi_{z} \Phi^{T} d x d y d z \\
& Q_{1}=\int_{\Omega} \Psi_{x} \Phi^{T} d x d y d z, \quad Q_{2}=\int_{\Omega} \Psi_{y} \Phi^{T} d x d y d z, \\
& F_{1}=\oint \Psi\left(\sigma_{11} n_{1}+\sigma_{12} n_{2}+\sigma_{13} n_{3}\right) d s \\
& F_{2}=\oint \Psi\left(\sigma_{21} n_{1}+\sigma_{22} n_{2}+\sigma_{23} n_{3}\right) d s \\
& F_{3}=\oint \Psi\left(\sigma_{31} n_{1}+\sigma_{32} n_{2}+\sigma_{33} n_{3}\right) d s
\end{aligned}
$$

For the consideration of using Gauss quadrature to calculate the above integrals, we transform the integrand from the global coordinate system $(x, y, z)$ into the local coordinate system $(\xi, \eta, \zeta)$, shown as

$$
\left[\begin{array}{c}
\Psi_{x}^{T} \\
\Psi_{y}^{T} \\
\Psi_{z}^{T}
\end{array}\right]=[J]^{-1}\left[\begin{array}{c}
\Psi_{\xi}^{T} \\
\Psi_{\eta}^{T} \\
\Psi_{\zeta}^{T}
\end{array}\right]=\left[\begin{array}{ccc}
J_{11}^{*} & J_{12}^{*} & J_{13}^{*} \\
J_{21}^{*} & J_{22}^{*} & J_{23}^{*} \\
J_{31}^{*} & J_{32}^{*} & J_{33}^{*}
\end{array}\right]\left[\begin{array}{c}
\Psi_{\xi}^{T} \\
\Psi_{\eta}^{T} \\
\Psi_{\zeta}^{T}
\end{array}\right]
$$

where $[J]$ is the Jacobian matrix and $J^{*}$ is the inverse of the Jacobian matrix. The 
Jacobian matrix is defined as

$$
\begin{aligned}
{[J] } & =\left[\begin{array}{lll}
J_{11} & J_{12} & J_{13} \\
J_{21} & J_{22} & J_{23} \\
J_{31} & J_{32} & J_{33}
\end{array}\right]=\left[\begin{array}{ccc}
\frac{\partial x}{\partial \xi} & \frac{\partial y}{\partial \xi} & \frac{\partial z}{\partial \xi} \\
\frac{\partial x}{\partial \eta} & \frac{\partial y}{\partial \eta} & \frac{\partial z}{\partial \eta} \\
\frac{\partial x}{\partial \zeta} & \frac{\partial y}{\partial \zeta} & \frac{\partial z}{\partial \zeta}
\end{array}\right] \\
& =\left[\begin{array}{lll}
\Psi_{\xi}^{T}\{x\} & \Psi_{\xi}^{T}\{y\} & \Psi_{\xi}^{T}\{z\} \\
\Psi_{\eta}^{T}\{x\} & \Psi_{\eta}^{T}\{y\} & \Psi_{\eta}^{T}\{z\} \\
\Psi_{\zeta}^{T}\{x\} & \Psi_{\zeta}^{T}\{y\} & \Psi_{\zeta}^{T}\{z\}
\end{array}\right]=\left[\begin{array}{c}
\Psi_{\xi}^{T} \\
\Psi_{\eta}^{T} \\
\Psi_{\zeta}^{T}
\end{array}\right]\left[\begin{array}{lll}
\{x\} & \{y\} & \{z\}
\end{array}\right]
\end{aligned}
$$

Therefore, in the local coordinate system, the stiffness matrix is defined as

$$
\begin{aligned}
& K_{11}=\mu \int_{\Omega}\left(J_{11}^{*} \Psi_{\xi}^{T}+J_{12}^{*} \Psi_{\eta}^{T}+J_{13}^{*} \Psi_{\zeta}^{T}\right)^{T}\left(J_{11}^{*} \Psi_{\xi}^{T}+J_{12}^{*} \Psi_{\eta}^{T}+J_{13}^{*} \Psi_{\zeta}^{T}\right) J d \xi d \eta d \zeta \\
& K_{22}=\mu \int_{\Omega}\left(J_{21}^{*} \Psi_{\xi}^{T}+J_{22}^{*} \Psi_{\eta}^{T}+J_{23}^{*} \Psi_{\zeta}^{T}\right)^{T}\left(J_{21}^{*} \Psi_{\xi}^{T}+J_{22}^{*} \Psi_{\eta}^{T}+J_{23}^{*} \Psi_{\zeta}^{T}\right) J d \xi d \eta d \zeta \\
& K_{33}=\mu \int_{\Omega}\left(J_{31}^{*} \Psi_{\xi}^{T}+J_{32}^{*} \Psi_{\eta}^{T}+J_{33}^{*} \Psi_{\zeta}^{T}\right)^{T}\left(J_{31}^{*} \Psi_{\xi}^{T}+J_{32}^{*} \Psi_{\eta}^{T}+J_{33}^{*} \Psi_{\zeta}^{T}\right) J d \xi d \eta d \zeta \\
& K_{21}=\mu \int_{\Omega}\left(J_{21}^{*} \Psi_{\xi}^{T}+J_{22}^{*} \Psi_{\eta}^{T}+J_{23}^{*} \Psi_{\zeta}^{T}\right)^{T}\left(J_{11}^{*} \Psi_{\xi}^{T}+J_{12}^{*} \Psi_{\eta}^{T}+J_{13}^{*} \Psi_{\zeta}^{T}\right) J d \xi d \eta d \zeta \\
& K_{12}=\mu \int_{\Omega}\left(J_{11}^{*} \Psi_{\xi}^{T}+J_{12}^{*} \Psi_{\eta}^{T}+J_{13}^{*} \Psi_{\zeta}^{T}\right)^{T}\left(J_{21}^{*} \Psi_{\xi}^{T}+J_{22}^{*} \Psi_{\eta}^{T}+J_{23}^{*} \Psi_{\zeta}^{T}\right) J d \xi d \eta d \zeta \\
& K_{31}=\mu \int_{\Omega}\left(J_{31}^{*} \Psi_{\xi}^{T}+J_{32}^{*} \Psi_{\eta}^{T}+J_{33}^{*} \Psi_{\zeta}^{T}\right)^{T}\left(J_{11}^{*} \Psi_{\xi}^{T}+J_{12}^{*} \Psi_{\eta}^{T}+J_{13}^{*} \Psi_{\zeta}^{T}\right) J d \xi d \eta d \zeta \\
& K_{13}=\mu \int_{\Omega}\left(J_{11}^{*} \Psi_{\xi}^{T}+J_{12}^{*} \Psi_{\eta}^{T}+J_{13}^{*} \Psi_{\zeta}^{T}\right)^{T}\left(J_{31}^{*} \Psi_{\xi}^{T}+J_{32}^{*} \Psi_{\eta}^{T}+J_{33}^{*} \Psi_{\zeta}^{T}\right) J d \xi d \eta d \zeta \\
& K_{23}=\mu \int_{\Omega}\left(J_{21}^{*} \Psi_{\xi}^{T}+J_{22}^{*} \Psi_{\eta}^{T}+J_{23}^{*} \Psi_{\zeta}^{T}\right)^{T}\left(J_{31}^{*} \Psi_{\xi}^{T}+J_{32}^{*} \Psi_{\eta}^{T}+J_{33}^{*} \Psi_{\zeta}^{T}\right) J d \xi d \eta d \zeta \\
& K_{32}=\mu \int_{\Omega}\left(J_{31}^{*} \Psi_{\xi}^{T}+J_{32}^{*} \Psi_{\eta}^{T}+J_{33}^{*} \Psi_{\zeta}^{T}\right)^{T}\left(J_{21}^{*} \Psi_{\xi}^{T}+J_{22}^{*} \Psi_{\eta}^{T}+J_{23}^{*} \Psi_{\zeta}^{T}\right) J d \xi d \eta d \zeta \\
& Q_{1}=\int_{\Omega}\left(J_{11}^{*} \Psi_{\xi}^{T}+J_{12}^{*} \Psi_{\eta}^{T}+J_{13}^{*} \Psi_{\zeta}^{T}\right)^{T} \Phi^{T} J d \xi d \eta d \zeta \\
& Q_{2}=\int_{\Omega}\left(J_{21}^{*} \Psi_{\xi}^{T}+J_{22}^{*} \Psi_{\eta}^{T}+J_{23}^{*} \Psi_{\zeta}^{T}\right)^{T} \Phi^{T} J d \xi d \eta d \zeta \\
& Q_{3}=\int_{\Omega}\left(J_{31}^{*} \Psi_{\xi}^{T}+J_{32}^{*} \Psi_{\eta}^{T}+J_{33}^{*} \Psi_{\zeta}^{T}\right)^{T} \Phi^{T} J d \xi d \eta d \zeta
\end{aligned}
$$


From the Gauss theorem, the surface integration $\mathbf{F}$ is

$$
\begin{aligned}
F_{1} & =\oint \Psi\left(\sigma_{11} n_{1}+\sigma_{12} n_{2}+\sigma_{13} n_{3}\right) d s \\
& =\sigma_{11} \int_{\Omega} \Psi_{x} d x d y d z+\sigma_{12} \int_{\Omega} \Psi_{y} d x d y d z+\sigma_{13} \int_{\Omega} \Psi_{z} d x d y d z \\
F_{2} & =\oint \Psi\left(\sigma_{21} n_{1}+\sigma_{22} n_{2}+\sigma_{23} n_{3}\right) d s \\
& =\sigma_{21} \int_{\Omega} \Psi_{x} d x d y d z+\sigma_{22} \int_{\Omega} \Psi_{y} d x d y d z+\sigma_{23} \int_{\Omega} \Psi_{z} d x d y d z \\
F_{3} & =\oint \Psi\left(\sigma_{31} n_{1}+\sigma_{32} n_{2}+\sigma_{33} n_{3}\right) d s \\
& =\sigma_{31} \int_{\Omega} \Psi_{x} d x d y d z+\sigma_{32} \int_{\Omega} \Psi_{y} d x d y d z+\sigma_{33} \int_{\Omega} \Psi_{z} d x d y d z
\end{aligned}
$$

In the local natural coordinate system,

$$
\begin{aligned}
F_{1} & =\sigma_{11} \int_{\Omega}\left(J_{11}^{*} \Psi_{\xi}^{T}+J_{12}^{*} \Psi_{\eta}^{T}+J_{13}^{*} \Psi_{\zeta}^{T}\right)^{T} J d \xi d \eta d \zeta \\
& +\sigma_{12} \int_{\Omega}\left(J_{21}^{*} \Psi_{\xi}^{T}+J_{22}^{*} \Psi_{\eta}^{T}+J_{23}^{*} \Psi_{\zeta}^{T}\right)^{T} J d \xi d \eta d \zeta \\
& +\sigma_{13} \int_{\Omega}\left(J_{31}^{*} \Psi_{\xi}^{T}+J_{32}^{*} \Psi_{\eta}^{T}+J_{33}^{*} \Psi_{\zeta}^{T}\right)^{T} J d \xi d \eta d \zeta \\
F_{2} & =\sigma_{21} \int_{\Omega}\left(J_{11}^{*} \Psi_{\xi}^{T}+J_{12}^{*} \Psi_{\eta}^{T}+J_{13}^{*} \Psi_{\zeta}^{T}\right)^{T} J d \xi d \eta d \zeta \\
& +\sigma_{22} \int_{\Omega}\left(J_{21}^{*} \Psi_{\xi}^{T}+J_{22}^{*} \Psi_{\eta}^{T}+J_{23}^{*} \Psi_{\zeta}^{T}\right)^{T} J d \xi d \eta d \zeta \\
& +\sigma_{23} \int_{\Omega}\left(J_{31}^{*} \Psi_{\xi}^{T}+J_{32}^{*} \Psi_{\eta}^{T}+J_{33}^{*} \Psi_{\zeta}^{T}\right)^{T} J d \xi d \eta d \zeta \\
F_{3} & =\sigma_{31} \int_{\Omega}\left(J_{11}^{*} \Psi_{\xi}^{T}+J_{12}^{*} \Psi_{\eta}^{T}+J_{13}^{*} \Psi_{\zeta}^{T}\right)^{T} J d \xi d \eta d \zeta \\
& +\sigma_{32} \int_{\Omega}\left(J_{21}^{*} \Psi_{\xi}^{T}+J_{22}^{*} \Psi_{\eta}^{T}+J_{23}^{*} \Psi_{\zeta}^{T}\right)^{T} J d \xi d \eta d \zeta \\
& +\sigma_{33} \int_{\Omega}\left(J_{31}^{*} \Psi_{\xi}^{T}+J_{32}^{*} \Psi_{\eta}^{T}+J_{33}^{*} \Psi_{\zeta}^{T}\right)^{T} J d \xi d \eta d \zeta
\end{aligned}
$$




\section{A.2.4 Gauss quadrature for tetrahedral element}

The Gauss quadrature are widely used for the integration in the Finite Element

Method. In this section, the Gauss quadrature for tetrahedral meshes is presented in Refs.[106-108].

$$
\begin{aligned}
\int_{\Omega} f(\xi, \eta, \zeta) d x d y d z & =\int_{\Omega} f(\xi, \eta, \zeta) J d \xi d \eta d \zeta=\int_{0}^{1} \int_{0}^{1-\zeta} \int_{0}^{1-\eta-\zeta} f(\xi, \eta, \zeta) J d \xi d \eta d \zeta \\
& =\frac{1}{6} \Sigma_{i=1}^{N} f\left(\xi_{i}, \eta_{i}, \zeta_{i}\right) J W_{i}
\end{aligned}
$$

where $N$ is the required number of Gauss points, $\xi_{i}, \eta_{i}, \zeta_{i}$ are the Gauss integration points, and $W_{i}$ is the Gauss weighting factors. Note that only one summation is needed for the area or volume integration in a triangular/tetrahedral mesh. 


\section{Bibliography}

[1] R. M. Jones. Mechanics of Composite Materials. Taylor and Francis, Inc., second edition, 1999.

[2] Chung, S. T. and Kwon, T. H. Coupled analysis of injection molding filling and fiber orientation, including in-plane velocity gradient effect. Polymer Composites, 17(6):859-872, 1996.

[3] Jack, D. A. and Smith, D. E. Elastic properties of short-fiber polymer composites, derivation and demonstration of analytical forms for expectation and variance from orientation tensors. Journal of Composite Materials, 42(3):277$308,2008$.

[4] Camacho, C. W., Tucker III, C. L., Yalvac, S., and McGee, R. L. Stiffness and thermal expansion predictions for hybrid short fiber composites. Polymer Composites, 11(4):229-239, 1990.

[5] Tucker III, C. L. and Liang, E. Stiffness predictions for unidirectional shortfiber composites: Review and evaluation. Composites Science and Technology, 59:655-671, 1999.

[6] Hatta, H. and Yamashita, S. Fiber orientation control by means of magnetic moment. Journal of Composite Materials, 22:484-500, 1998. 
[7] A. Einstein. Berichtigung zu meiner arbeit: Eine neue bestimmung der molekldimensionen. Annalen der Physik, 339(3):591-592, 1911.

[8] Jeffery, G. B. The motion of ellipsoidal particles immersed in a viscous fluid. Proceedings of the Royal Society of London. Series A, 102(715):161-179, 1922.

[9] Lamb, H. Hydrodynamics. Cambridge university press, 1916.

[10] Folgar, F. and Tucker III, C. L. Orientation behavior of fibers in concentrated suspensions. Journal of Reinforced Plastics and Composites, 3(2):98-119, 1984.

[11] Junk, M. and Illner, R. A new derivation of jeffery's equation. Journal of Mathematical Fluid Mechanics, 9(4):445-288, 2007.

[12] Advani, S. G. and Tucker III, C. L. The use of tensors to describe and predict fiber orientation in short fiber composites. Journal of Rheology, 31(8):751-784, 1987.

[13] Cintra, J. S. and Tucker III, C. L. Orthotropic closure approximations for flow-induced fiber orientation. Journal of Rheology, 39(6):1095-1122, 1995.

[14] Montgomery-Smith, S., Jack, D. A., and Smith, D. E. The fast exact closure for jeffery's equation with diffusion. Journal of Non-Newtonian Fluid Mechanics, $166(7-8): 343-353,2011$.

[15] Doi, M. Molecular dynamics and rheological properties of concentrated solutions of rodlike polymers in isotropic and liquid crystalline phases. Journal of Polymer Science: Part B, 19:229-243, 1981.

[16] Verleye, V. and Dupret, F. Prediction of fiber orientation in complex injection molded parts. In Developments in Non-Newtonian Flows, pages 139-163, 1993. 
[17] Dupret, F., Verleye, V., and Languillier, B. Numerical prediction of the molding of composite parts. In Rheology and Fluid Mechanics of Nonlinear Materials, pages 79-90, 1997.

[18] Jack, D. A. and Smith, D. E. An invariant based fitted closure of the sixth-order orientation tensor for short-fiber suspensions. Journal of Rheology, 49(5):1091$1115,2005$.

[19] Petrie, C. J. S. The rheology of fibre suspensions. Journal of Non-Newtonian Fluid Mechanics, 87(2-3):369-402, 1999.

[20] Leal, L. G. Particle motions in a viscous fluid. Annual Review of Fluid Mechanics, 12(1):435-476, 1980.

[21] Feng, J. and Joseph, D. D. The unsteady motion of solid bodies in creeping flows. Journal of Fluid Mechanics, 303:83-102, 1995.

[22] Barbosa, S. E. and Bibbo, M. A. Fiber motion and rheology of suspensions with uniform fiber orientation. Journal of Polymer Science: Part B, 38:1788-1799, 2000.

[23] Jia, L. Flow-induced Alignment Migration of Particles in Suspensions. Ph.d. thesis, Michigan State University, 2006.

[24] Batchelor, G. K. The stress system in a suspension of force-free particles. Journal of Fluid Mechanics, 41:545-570, 1970.

[25] Batchelor, G. K. Slender body theory for particles of arbitrary cross-section in stoke flow. Journal of Fluid Mechanics, 44:419-440, 1970.

[26] Yamamoto, S. and Matsuoka, T. A method for dynamic simulation of rigid and flexible fibers in a flow field. Journal of Chemical Physics, 98(1):644-650, 1993. 
[27] Ross, R. F. and Klingenberg, D. J. Dynamic simulation of flexible fibers composed of linked rigid bodies. Journal of Chemical Physics, 106(7):2949-2960, 1997.

[28] Wang, G., Yu, W., and Zhou, C. Optimization of the rod chain model to simulate the motions of a long flexible fiber in simple shear flows. European Journal of Mechanics - B/Fluids, 25(3):337-347, 2006.

[29] Switzer III, L. H. Simulating Systems of Flexible Fibers. Ph.d. thesis, University of Wisconsin-Madison, 2002.

[30] Taylor, G. I. The motion of ellipsoidal particles in a viscous fluid. Proceedings of the Royal Society of London. Series A, 103(720):58-61, 1923.

[31] Trevelyan, B. J. and Mason, S. G. Particle motions in sheared suspensions. i. rotations. Journal of Colloid Science, 6:354-367, 1951.

[32] Mason, S. G. and Manley, R. St J. Particle motions in sheared suspensions: Orientation and interactions of rigid rods. Proceedings of the Royal Society of London. Series A, 238(1212):117-131, 1956.

[33] Anczurowski, E. and Mason, S. G. Particle motions in sheared suspensions. xxiv. rotation of rigid spheroids and cylinders. Journal of Rheology, 12(2):209$215,1968$.

[34] Wang, J., O'Gara, J. F., and Tucker III, C. L. An objective model for slow orientation kinetics in concentrated fiber suspensions: Theory and rheological evidence. Journal of Rheology, 52(5):1179-1200, 2008.

[35] TuckerIII, C. L., Wang, J., O'Gara, J. F., and Debarr, G. Improved fiber orientation predictions for injection molded composites. Technical report, University of Illinois-Champaign and Delphi Research Labs, June 2004. 
[36] Phan-Thien, N., Fan, X. J., Tanner, R. I., and Zheng, R. Folgar-tucker constant of a fibre suspension in a newtonian fluid. Jounal of Non-Newtonian Fluid Mechanics, 103:251-260, 2002.

[37] Fan, X. J., Phan-Thien, N., and Zheng, R. A direct simulation of fibre suspensions. Journal of Non-Newtonian Fluid Mechanics, 74(1-3):113-135, 1998.

[38] Bretherton, F. P. The motion of rigid particles in a shear flow at low reynolds number. Journal of Fluid Mechanics, 14(2):284-304, 1962.

[39] Petrich, M. P., Koch, D. L., and Cohen, C. An experimental determination of the stress-microstructure relationship in semi-concentrated fiber suspension. Journal of Non-Newtonian Fluid Mechanics, 95(2-3):101-133, 2000.

[40] Harris, J. B. and Pittman, J. F. T. Equivalent ellipsoidal aspect ratios of slender rod-like particles. Journal of Colloid and Interface Science, 50(2):280-282, 1975.

[41] Cox, R. G. The motion of long slender bodies in a viscous fluid. part2. shear flow. Journal of Fluid Mechanics, 45(4):625-657, 1971.

[42] Chapra, S. C. Applied Numerical Methods with MATLAB for Engineers and Scientists-Second Edition. McGraw-Hill, 2008.

[43] Chaffey, C. E., Takano, M., and Mason, S. G. Particle motions in sheared suspensions. xvi. orientations of rods and disks in hyperbolic and other flows. Canadian Journal of Physics, 43:1269-1287, 1965.

[44] Segré, G. and Silberberg, A. Radial particle displacements in poiseuille flow of suspensions. Nature, 189:209-210, 1961.

[45] Goldsmith, H. L. and Mason, S. G. Axial migration of particles in poiseuille flow. Nature, 194:1095-1096, 1961. 
[46] Karnis, A., Goldsmith, H. L, and Mason, S. G. Axial migration of particles in poiseuille flow. Nature, 200:159-160, 1963.

[47] Karnis, A., Goldsmith, H. L., and Mason, S. G. The flow of suspensions through tubes, v. inertial effects. The Canadian Journal of Chemical Engineering, 44(4):181-193, 1966.

[48] Oliver, D. R. Influence of particle rotation on radial migration in the poiseuille flow of suspensions. Nature, 194:1269-1271, 1962.

[49] Repetti, R. V. and Leonard, E. F. Segré-silberberg annulus formation: A possible explanation. Nature, 203:1346-1348, 1964.

[50] Rubinow, S. I. and Keller, Joseph B. The transverse force on a spinning sphere moving in a viscous fluid. Journal of Fluid Mechanics, 11(3):447-459, 1961.

[51] Saffman, P. G. The lift on a small sphere in a slow shear flow. Journal of Fluid Mechanics, 22:385-400, 1965.

[52] Cox, R. G. and Brenner, H. The lateral migration of solid particles in poiseuillei theory. Chemical Engineering Science, 23:147-17, 1968.

[53] Ho, B. P. and Leal, L. G. Inertial migration of rigid spheres in two-dimensional unidirectional flows. Journal of Fluid Mechanics, 65(part 2):365-400, 1974.

[54] Tachibana, M. On the behavior of a sphere in the laminar tube flows. Rheologica Acta, 12(1):1346-1348, 1973.

[55] Vasseur, P. and Cox, R. G. The lateral migration of a spherical particle in two-dimensional shear flows. Journal of Fluid Mechanics, 78(part 2):385-413, 1976.

[56] Schonberg, J. and Hinch, E. J. Inertial migration of a sphere in poiseuille flow. Journal of Fluid Mechanics, 203:517-524, 1989. 
[57] Chwang, A. T. Hydromechanics of low-reynolds-number flow. part3. motion of a spheroidal particle in quadratic flows. Journal of Fluid Mechanics, 72(1):17-34, 1975 .

[58] Stover, C. A. and Cohen, C. The motion of rodlike particles in the pressuredriven flow between two flat plates. Rheologica Acta, 29:192-203, 1990.

[59] Sugihara-Seki, M. The motion of an elliptical cylinder in channel flow at low reynolds numbers. Journal of Fluid Mechanics, 257:575-596, 1993.

[60] Sugihara-Seki, M. The motion of an ellipsoid in tube flow at low reynolds numbers. Journal of Fluid Mechanics, 324:287-308, 1996.

[61] Feng, J., Hu, H. H., and Joseph, D. D. Direction simulation of initial value problems for the motion of solid bodies in a newtonian fluid, part2. couette and poiseuille flows. Journal of Fluid Mechanics, 277:271-301, 1994.

[62] Hu, H. H., Joseph, D. D., and Crochet, M. J. Direct simulation of fluid particle motions. Theoretical and Computational Fluid Dynamics, 3:285-306, 1992.

[63] Clague, D. S. and Cornelius, P. J. The hydrodynamic force and torque on a bounded sphere in poiseuille flow. International Journal for Numerical Methods in Fluids, 35:55-70, 2001.

[64] Reddy, J. N. and Gartling, D. K. The finite element method in heat transfer and fluid dynamics, Second edition. CRC Press LLC, 2001.

[65] Donea, J. and Huerta, A. Finite Element Methods for Flow Problems. John Wiley \& Sons Ltd, 2003.

[66] Qian, D., Dickey, E. C., Andrews, R., and Rantell, T. Load transfer and deformation mechanisms in carbon nanotube-polystyrene composites. Applied Physics Letter, 76(20):2868-70, 2000. 
[67] Biercuk, M. J., Llaguno, M. C., Radosavljevic, M., Hyun, J. K., Johnson, A. T., and Fischer, J. E. Carbon nanotube composites for thermal management. Applied Physics Letter, 80(15):2767-2769, 2002.

[68] Hinch, E. J. and Leal, L. G. The effect of brownian motion on the rheological properties of a suspension of non-spherical particles. Journal of Fluid Mechanics, 52:683-712, 1972.

[69] Hinch, E. J. and Leal, L. Constitutive equations in suspension mechanics. part 2. approximate forms for a suspension of rigid particles affected by brownian rotations. Journal of Fluid Mechanics, 76(1):187-208, 1976.

[70] Tao, Y., den Otter, W. K., Padding, J. T., Dhont, J. K. G., and Briels, W. J. Brownian dynamics simulations of the self- and collective rotational diffusion coefficients of rigid long thin rods. Journal of Chemical Physics, 122(24):1-10, 2005.

[71] Yamamoto, T. and Kasama, H. Brownian dynamics simulation of multiphase suspension of disc-like particles and polymers. Rheologica Acta, 49(6):573-584, 2010.

[72] Meng, Q. and Higdon, J. J. L. Large scale dynamics simulation of plate-like particle suspensions. part ii: Brownian simulation. Journal of Rheology, 52(1):37$65,2008$.

[73] Somasi, M., Khomami, B., Woo, N. J., Hur, J. S., and Shaqfeh, E. S. G. Brownian dynamics simulations of bead-rod and bead-spring chains: Numerical algorithms and coarse-graining issues. Journal of Non-Newtonian Fluid Mechanics, 108(1-3):227-255, 2002.

[74] Tang, W. and Advani, S. G. Dynamic simulation of carbon nanotubes in simple 
shear flow. Computer Modeling in Engineering and Sciences, 25(3):149-164, 2008.

[75] Ermak, D. L. and Buckholz, H. Numerical integration of langevin equation: Monte carlo simulation. Journal of Computational Physics, 35:169-182, 1980.

[76] Kim, S. and Karrila, S. J. Microhydrodynamics: Principles and Selected Applications. Butterworth-Heinemann, 1991.

[77] Stover, C. A., Koch, D. L., and Cohen, C. Observations of fibre orientation in simple shear flow of semi-dilute suspensions. Journal of Fluid Mechanics, 238:277-296, 1992.

[78] Dinh, S. M. and Armstrong, R. C. Rheological equation of state for semiconcentrated fiber suspensions. Journal of Rheology, 28(3):207-227, 1984.

[79] Lipscomb, G. G., Denn, M. M., Hur, D. U., and Boger, D. V. The flow of fiber suspensions in complex geometries. Journal of Non-Newtonian Fluid Mechanics, 26:297-325, 1988.

[80] Mackaplow, M. B. and Shaqfeh, S. G. A numerical study of the rheological properties of suspensions of rigid, non-brownian fibres. Journal of Fluid Mechanics, 329:155-186, 1996.

[81] Rahnama, M. and Shaqfeh, S. G. The effect of hydrodynamic interactions on the orientation distribution in a fiber suspension subject to simple shear flow. Physics of Fluids, 7(3):487-506, 1995.

[82] Yamane, Y., Kaneda, Y., and Dio, M. Numerical simulation of semi-dilute suspensions of rodlike particles in shear flow. Journal of Non-Newtonian Fluid Mechanics, 54:405-421, 1994. 
[83] Claeys, I. L. and Brady, J. F. Suspensions of prolate spheroids in stokes flow. part 1. dynammics of a finite number of particles in an unbounded fluid. Journal of Fluid Mechanics, 251:411-442, 1993.

[84] Jayageeth, C., Sharma, V. I., and Singh, A. Dynamic of short fiber suspensions in bounded shear flow. International Journal of Multiphase Flow, 35:261-269, 2009.

[85] Zhang, D., Smith, D. E., Jack, D. A., and Montgomery-Smith, S. Numerical evaluation of single fiber motion for short-fiber-reinforced composite materials processing. ASME Transactions Journal of Manufacturing and Science Engineering, 131(1):1-9, 2011.

[86] Batchelor, G. K. An Introduction to Fluid Dynamics. Cambridge University Press, 1967.

[87] Durbin, P. A. and Medic, G. Fluid Dynamics with a Computational Perspective. Cambridge University Press, 2007.

[88] Zhang, D. and Smith, D. E. Finite element-based brownian dynamics simulation of nano-fiber suspensions in nano-composites processing using monte-carlo method. In Proceedings of the ASME International Mechanical Engineering Congress \& Exposition, 2012.

[89] Zhang, D., Smith, D. E., Jack, D. A., and Montgomery-Smith, S. Rheological study on multiple fiber suspensions for fiber reiforced composite materials processing. In Proceedings of the ASME International Mechanical Engineering Congress \& Exposition, 2011.

[90] Harlen, O. G., Sundararajakumar, R. R., and Koch, D. L. Numerical simulations of a sphere settling through a suspension of neutrally buoyant fibers. Journal of Fluid Mechanics, 388:355-388, 1999. 
[91] L.F. Carter. A study of the rheology of suspensions of rod-shaped particles in a Navier-Stokes liquid. Ph.d. thesis, University of Michigan-Ann Arbor, 1967.

[92] Bennington, C., Kerekes, R., and Grace, J. The yield stress of fibre suspensions. Journal of Chemical Engineering, 68:748-757, 1990.

[93] Wang, W., Wang, J., and Kim, M. An algebraic condition for the separation of two ellipsoids. Computer Aided Geometric Design, 18:531-539, 2001.

[94] Choi, Y., Chang, J., Wang, W., Kim, M., and Elber, G. Continuous collision detection for ellipsoids. IEEE Transactions on Visualization and Computer Graphics, 15(2):311-322, 2009.

[95] Joung, G. G., Phan-Thien, N., and Fan, X. J. Direct simulation of flexible fibers. Journal of Non-Newtonian Fluid Mechanics, 99:1-36, 2001.

[96] Joung, G. G., Phan-Thien, N., and Fan, X. J. Viscosity of curled fibres in suspension. Journal of Non-Newtonian Fluid Mechanics, 102:1-17, 2002.

[97] Skjetne, P., Ross, R. F., and Klingenberg, D. J. Simulation of single fiber dynamics. Journal of Chemical Physics, 107(6):2108-2121, 1997.

[98] Yamamoto, S. and Matsuoka, T. Dynamic simulation of microstructure and rheology of fiber suspensions. Polymer Engineering and Science, 36(19):23962403, 1996.

[99] Advani, S. G. and Sozer, E. M. Process modeling in composites manufacturing. Marcel Dekker, Inc., 2003.

[100] Goto, S., Nagazono, H, and Kato, H. The flow behavior of fiber suspensions in newtonian fluids and polymer solutions. i. mechanical properties. Rheologica Acta, 25:119-129, 1986. 
[101] Tadmor, Z. and Gogos, C. G. Principles of polymer processing. John Wiley \& Sons Ltd, 2006.

[102] Ganani, E. and Powell, R. L. Rheological properties of rodlike particles in a newtonian and a non-newtonian fluid. Journal of Rheology, 30(5):995-1013, 1986.

[103] Chan, Y., White, J. L., and Oyanagi, Y. A fundamental study of the rheological properties of glass-fiber-reinforced polythylene and polystyrene melts. Journal of Rheology, 22(5):507-524, 1978.

[104] Phan-Thien, N. and Fan, X. Viscoelastic mobility problem using a boundary element method. Journal of Non-Newtonian Fluid Mechanics, 105(2-3):131$152,2002$.

[105] Goddard, J. D. Tensile stress contribution of flow-oriented slender particles in non-newtonian fluids. Journal of Non-Newtonian Fluid Mechanics, 1:1-17, 1976.

[106] Yu, J. Symmetric gaussian quadrature formulae for tetrahedronal regions. Computer Methods in Applied Mechanics and Engineering, 43:349-353, 1984.

[107] Dunavant, D. A. High degree efficient symmetrical gaussian quadrature rules for the triangle. International Journal of Numerical Methods in Engineering, 21:1129-1148, 1985.

[108] Keast, P. Moderate-degree tetrahedral quadrature formulas. Computer Methods in Applied Mechanics and Engineering, 55:339-348, 1986. 


\section{VITA}

Dongdong Zhang earned both of his Bachelor and Master degrees in Mechanical Engineering at the Hefei University of Technology in 2004 and 2007, respectively. From August 2007 to December 2008, he studied at the Illinois Institute of Technology in Chicago. His research work included Computer-Aided Design and Manufacturing (CAD/CAM), Geometric/Solid Modeling, Point-Based Computational Techniques and Nano-scale Imaging. In January 2009, Dongdong Zhang joined the Department of Mechanical and Aerospace Engineering at the University of Missouri for the doctoral degree. During his Ph.D. study, he worked as a research and teaching assistant

under the supervision of Dr. Douglas E. Smith. Dongdong Zhang's research focused on Finite Element Analysis (FEA), Computational Fluid Dynamics (CFD), Numerical Algorithms and Flow-Induced Fiber Suspensions Simulation. After graduation, he moved to Dallas-Fort Worth area, Texas.

Dongdong Zhang got married with Na Deng in 2008 and had their first son, Jayden in 2012. 